Isabelle Ihring

Budrich

UniPress

Weibliche Genital-

beschneidung im

Kontext von Migration 


\section{Isabelle Ihring}

Weibliche Genitalbeschneidung im Kontext von

Migration 


\author{
Isabelle Ihring
}

\title{
Weibliche Genitalbeschneidung im Kontext von Migration
}

Budrich UniPress Ltd.

Opladen • Berlin • Toronto 2015 
Bibliografische Information der Deutschen Nationalbibliothek

Die Deutsche Nationalbibliothek verzeichnet diese Publikation in der Deutschen Nationalbibliografie; detaillierte bibliografische Daten sind im Internet über http://dnb.d-nb.de abrufbar.

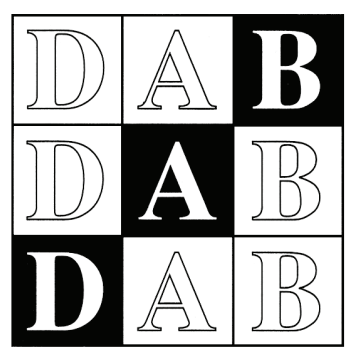

Die Drucklegung dieser Publikation wurde gefördert vom Deutschen Akademikerinnenbund (DAB).

Gedruckt auf säurefreiem und alterungsbeständigem Papier.

Alle Rechte vorbehalten.

(C) 2015 Budrich UniPress, Opladen, Berlin \& Toronto

www.budrich-unipress.de

$$
\begin{array}{ll}
\text { ISBN } & 978-3-86388-707-0 \text { (Paperback) } \\
\text { eISBN } & 978-3-86388-264-8 \\
\text { (eBook) }
\end{array}
$$

Das Werk einschließlich aller seiner Teile ist urheberrechtlich geschützt. Jede Verwertung außerhalb der engen Grenzen des Urheberrechtsgesetzes ist ohne Zustimmung des Verlages unzulässig und strafbar. Das gilt insbesondere für Vervielfältigungen, Übersetzungen, Mikroverfilmungen und die Einspeicherung und Verarbeitung in elektronischen Systemen.

Umschlaggestaltung: Bettina Lehfeldt, Kleinmachnow - http://www.lehfeldtgraphic.de Lektorat: Judith Henning, Hamburg - www.buchfinken.com 


\section{Danksagung}

Die vorliegende Forschungsarbeit wäre nicht entstanden, hätten mir nicht zahlreiche Menschen zur Seite gestanden, mich unterstützt, beraten und mich immer wieder ermutigt, diese Arbeit zu schreiben. $\mathrm{Zu}$ allererst möchte ich mich bei meinen beiden Gutachter_innen Frau Prof. Dr. Christine Riegel und Herr Prof. Dr. Thomas Fuhr für die Betreuung und Begleitung bedanken. Besonderer Dank geht an Frau Prof. Dr. Christine Riegel, die mich geduldig, aufmunternd und immer motivierend durch diesen teilweise zermürbenden Prozess begleitet hat.

Mein ausgesprochener Dank geht außerdem an Marione Hulverscheidt, ohne die ich meine Idee nie umgesetzt hätte und die mich während des gesamten Forschungsprozesses und der Abschrift dieser Arbeit unglaublich unterstützt hat. Peter Heuschkel-Jokl danke ich, dass er mir nicht nur geduldig durch alle Hochs und Tiefs einer Dissertation zugehört hat, sondern am Ende mit seinem Blick auf meinen Text auch geholfen hat Schwachstellen aufzudecken. Ute Seckinger, Eva Breunig und Julia Weinhold danke ich dafür, dass sie die Entwicklung dieser Arbeit mitgetragen haben und mit ihrer Diskussionsbereitschaft dazu beigetragen haben, dass diese sich weiter entwickelt hat. Besonderer Dank geht auch an Thorsten Linder, Kerstin Brutschin und Christine Wallner. Meiner Kolloquiumsgruppe danke ich für zahlreiche Tipps, verschiedene Perspektiven und dafür, dass sie sich mit vielen Fragmenten dieser Arbeit auseinandergesetzt haben. Hawa Duale Fritsche, Asili Barre-Dirie, Fadumo Korn und Salahdin Maow danke ich für Übersetzungen, Vermittlung von Interviewpartner_innen und etliche Einblicke in die somalische Politik und Gesellschaft.

Ich danke von ganzem Herzen meinen Eltern Anton und Danielle Ihring, die immer an mich geglaubt haben. Besonders danke ich meiner Mutter, dass sie an diversen Stellen ihr Sprachtalent zur Verfügung gestellt hat. Ein großes Dankeschön geht außerdem an Matthias Linder, der mich in allen Phasen erlebt, ertragen und unterstützt hat. Und natürlich danke ich von Herzen meinem Sonnenschein Malia Mella Linder, die mich immer wieder ans Wesentliche im Leben erinnert und deshalb viele Täler erträglicher gemacht hat.

Abschließend gilt mein außerordentlicher Dank all denjenigen somalischen Frauen und Männern, die mir so viel Vertrauen entgegen gebracht haben und bereit waren, offen über dieses intime Thema zu sprechen. Ohne euch, hätte diese Arbeit nicht entstehen können. 



\section{Inhaltsverzeichnis}

$1 \quad$ Einleitung ...................................................................................................... 11

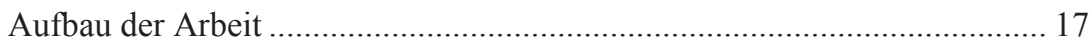

I Theoretischer Rahmen................................................................... 20

2 Weibliche Genitalbeschneidung..................................................... 20

2.1 Definition und Formen weiblicher Genitalbeschneidung..................... 21

2.2 Mögliche Konsequenzen weiblicher Beschneidung ............................. 22

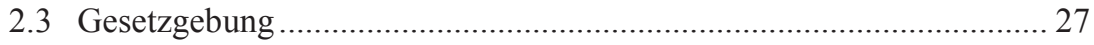

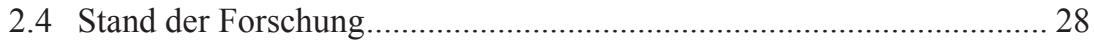

3 Zum politischen und gesellschaftlichen Kontext in Somalia und den Einwanderungsländern ....................................................... 35

3.1 Politische und gesellschaftliche Situation in Somalia .......................... 35

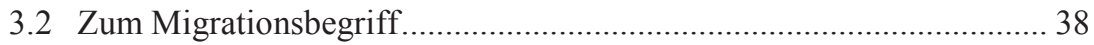

3.3 Zur Migrationspolitik Deutschlands.................................................. 39

3.4 Zur Migrationspolitik der Schweiz ....................................................... 42

3.5 Zur Migrationspolitik Englands ..................................................... 43

3.6 Zur Migrationspolitik Italiens .......................................................... 45

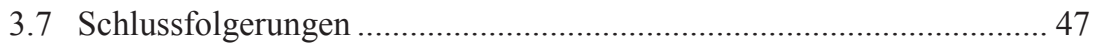

4 Theoretische Perspektiven................................................................. 49

4.1 Postkoloniale Theorien.......................................................................... 49

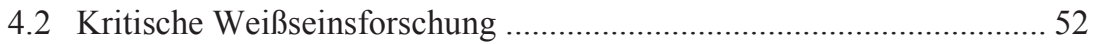

4.3 Feministische Perspektive und die Konstruktion von Geschlecht......... 56

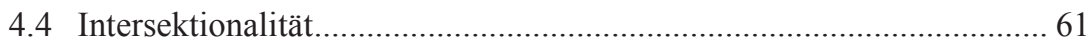

4.5 Exkurs: Zur Auseinandersetzung mit Kulturkonstruktionen................. 64 
1 Methodik und Durchführung der Studien ............................................ 70

1.1 Erkenntnisinteresse und Fragestellungen ........................................... 70

1.2 Zur Kombination quantitativer und qualitativer Forschung ................. 71

1.3 Methodisches Vorgehen bei der Befragung der Expert_innen.............. 73

1.4 Methodisches Vorgehen bei den qualitativen Interviews ...................... 76

1.5 Forschungszugang und Sample der qualitativen Untersuchung ............ 79

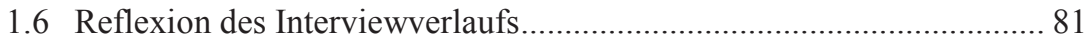

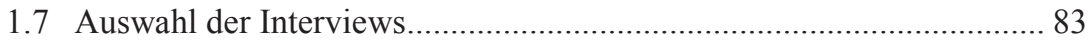

1.8 Analyse der Interviews in Anlehnung an die Grounded Theory .......... 85

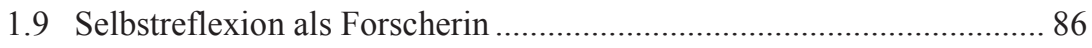

2 Ergebnisse der Befragung der Beratungsstellen ................................ 89

2.1 Zur Beratungssituation in Deutschland .............................................. 89

2.2 Zur Beratungssituation in der Schweiz ............................................. 91

2.3 Zur Beratungssituation in England.................................................... 93

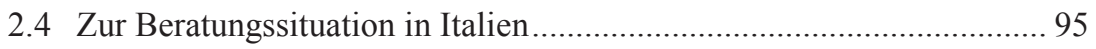

2.5 Zentrale Ergebnisse der Befragung .................................................... 96

3 Ergebnisse der migrierten Frauen somalischer Herkunft ................ 99

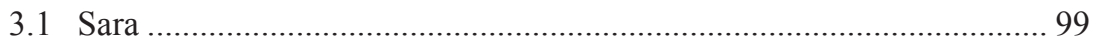

3.1.1 Unzureichende medizinische Versorgung in Somalia ............ 101

3.1.2 Sozialer Druck und der Wunsch nach Anpassung .................. 103

3.1.3 Vom Tag der Beschneidung ............................................... 106

3.1.4 Saras Erfahrungen in Deutschland......................................... 107

3.1.5 Vom Prozess des Umdenkens .............................................. 110

3.1.6 Saras Perspektive auf die Aufklärungs- und Beratungsarbeit in Deutschland .................................................................. 111

3.1.7 Saras Blick auf die Rolle der Männer im Kontext weiblicher Genitalbeschneidung................................................................ 113

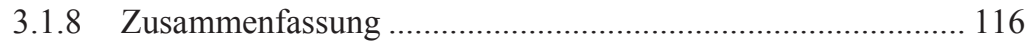

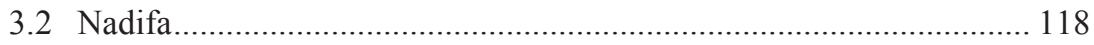

3.2.1 Nadifas Erfahrungen in der Schweiz ................................... 118 
3.2.2 Eltern haben die Entscheidungsmacht ................................. 120

3.2.3 Eine Frage der Haltung ........................................................ 122

3.2.4 Medizinisches Fachpersonal in der Schweiz ......................... 125

3.2.5 Zusammenfassung ............................................................. 126

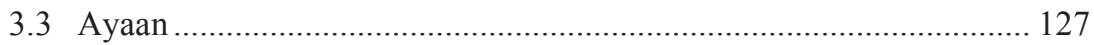

3.3.1 Weibliche Genitalbeschneidung in Somalia ......................... 128

3.3.2 Zu den Folgen der Infibulation .............................................. 131

3.3.3 Zusammenfassung ............................................................... 135

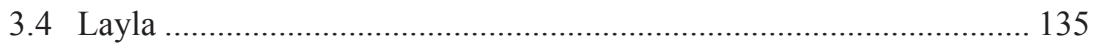

3.4.1 Ein Leben in Angst, Abhängigkeit und Schmerz.................... 136

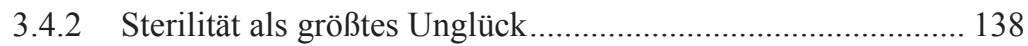

3.4.3 Zusammenfassung ……................................................... 140

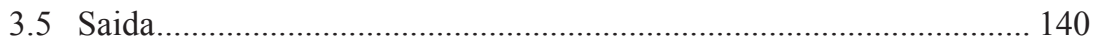

3.5.1 Auswirkungen einer den Eingriff problematisierenden

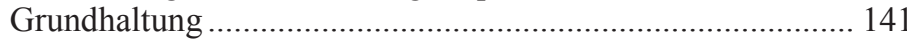

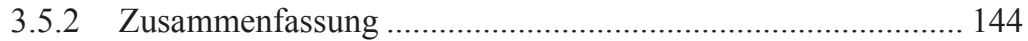

3.6 Zentrale Ergebnisse der Fallanalysen............................................. 145

3.6.1 Mädchenbescheidung wird als gesellschaftlich etablierte Norm in Somalia beschrieben ..................................................... 145

3.7 Migration wird sowohl als Einschnitt als auch Möglichkeit des

Perspektivwechsels erlebt .................................................................. 146

4 Ergebnisse der migrierten Männer somalischer Herkunft............ 153

4.1 Mädchenbeschneidung wird als ,normal' beschrieben ...................... 154

4.2 Aus Sicht der Männer tradieren Frauen die Praktik ............................ 155

4.3 Weibliche Genitalbeschneidung als eines von vielen Problemen ....... 158

4.4 Gespräche als Schlüssel im Prozess des Umdenkens .......................... 159

4.5 Zusammenfassung der zentralen Ergebnisse....................................... 160

5 Zusammenschau der Gesamtergebnisse ....................................... 163

5.1 Genitalbeschneidung als fester Bestandteil bei der Konstruktion von Weiblichkeit

5.2 Konsequenzen einer Migration in ein Genitalbeschneidung ablehnendes Einwanderungsland 166 
6 Konsequenzen für Politik und Beratung...................................... 169

6.1 Finanzielle Förderung von Migrant_innenvereinen ........................... 169

6.2 Aufnahme der Thematik in die medizinische, psychologische und pädagogische Ausbildung .................................................................. 169

6.3 Schulungen für Mediziner_innen, Psycholog innen und Pädagog_innen im Umgang mit beschnittenen Frauen ........................ 171

6.4 Offenheit gegenüber neuen Wegen in der Beratung ......................... 172

6.5 Beratung in Flüchtlingswohnheimen ................................................. 173

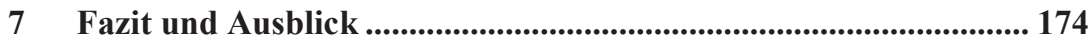

8 Transkriptionssystem ..................................................................... 177

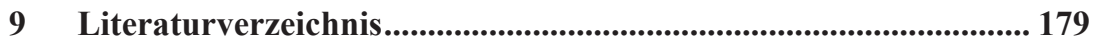




\section{Einleitung}

Die vorliegende Dissertation stellt das Phänomen der Genitalbeschneidung von Mädchen und Frauen in den Fokus und untersucht den Umgang mit beschnittenen oder von Beschneidung bedrohten Migrantinnen ${ }^{1}$ und Flüchtlingen $^{2}$ in verschiedenen europäischen Ländern. ${ }^{3}$ Bei weiblicher Genitalbeschneidung handelt es sich um eine Form der Gewalt an Mädchen und Frauen, die in der Convention on the Elimination of all Forms of Discrimination against Women (CEDAW) als Menschenrechtsverletzung explizit benannt ist (CEDAW General Recommondation No. 14: Female Circumcision) und von der Mehrzahl aller Länder als solche anerkannt wird. Trotz der weltweiten Anerkennung weiblicher Genitalbeschneidung als Menschenrechtsverletzung und der Tatsache, dass die Praktik in nahezu allen Ländern - auch denen, in denen Genitalbeschneidung weiterhin praktiziert wird - per Gesetz verboten ist, werden in weiten Teilen Afrikas, in manchen arabisch geprägten Ländern, verschiedenen Regionen Asiens und des Mittleren Ostens Mädchen und Frauen beschnitten (Terre des Femmes 2003, S. 59). Doch lässt sich weibliche Genitalbeschneidung nicht ausschließlich auf die genannten Regionen beschränken. Die Entwicklung der letzten Jahre zeigt, dass die Zahl der Menschen, die aus Genitalbeschneidung praktizierenden Ländern nach Deutschland und in andere europäische Länder immigrieren, kontinuierlich ansteigt, somit das Thema auch in Europa mehr und mehr an Brisanz gewinnt (Bun-

1 Gemäß der Definition des Bundesamtes für Migration und Flüchtlinge (BAMF) werden „[a]lle nach 1949 auf das heutige Gebiet der Bundesrepublik Deutschland Zugewanderten, sowie alle in Deutschland geborenen Ausländer und alle in Deutschland als Deutsche Geborenen mit zumindest einem zugewanderten oder als Ausländer in Deutschland geborenen Elternteil " als Migrant_in und/oder Menschen mit Migrationshintergrund bezeichnet.

2 Laut Artikel 1 der Genfer Flüchtlingskonvention ist ein Flüchtling eine „Person, die sich außerhalb des Landes befindet, dessen Staatsangehörigkeit sie besitzt oder in dem sie ihren ständigen Wohnsitz hat, und die wegen ihrer ,Rasse', Religion, Nationalität, Zugehörigkeit zu einer bestimmten sozialen Gruppe oder wegen ihrer politischen Überzeugung eine wohlbegründete Furcht vor Verfolgung hat und den Schutz dieses Landes nicht in Anspruch nehmen kann oder wegen dieser Furcht vor Verfolgung nicht dorthin zurückkehren kann.“ Hinter der Bezeichnung ,Migrant_in“ steht mehr als die bloße Definition, da ,Migration nicht nur als „dauerhafte Ortsveränderung“ (Reinprecht/Weiss 2012, S. 15) betrachtet und benannt werden kann, sondern vielmehr als „Gegenstand von Diskursen und als Gegenstand politischer und alltagsweltlicher Auseinandersetzung" (Mecheril 2004, S. 42) gesehen werden muss. Die Darstellung der damit verbundenen Diskurse und deren Auswirkungen ist Teil des zweiten Kapitels dieser Arbeit. 
desamt für Statistik 2013). Die Menschenrechtsorganisation Terre des Femmes e.V. schätzt für Deutschland die Zahl der betroffenen beziehungsweise bedrohten Mädchen und Frauen auf circa 25000 (Terres des Femmes 2013). Hinzu kommt die Vermutung, dass es Familien gibt, die auch in der Diaspora an der Praktik festhalten und Wege finden, ihre Töchter beschneiden zu lassen (Vgl. Behrendt 2010), ein Eingriff, der dann tendenziell eher im Heimatland durchgeführt wird (ebd., S.100). Daher stehen Deutschland und andere europäische Länder vor der Herausforderung, den von weiblicher Genitalbeschneidung betroffenen und bedrohten Mädchen und Frauen schützend und unterstützend $\mathrm{zu}$ begegnen. Das Ziel, mithilfe dieser Forschungsarbeit Erkenntnisse darüber zu gewinnen, wie in Deutschland lebende bedrohte Mädchen künftig vor dem Eingriff geschützt und bereits beschnittene Migrantinnen in ihren Bedürfnissen wahrgenommen und begleitet werden können, soll anhand von zwei Untersuchungen erreicht werden.

Bevor jedoch auf das konkrete Forschungsanliegen dieser Arbeit eingegangen werden soll, wird vorab die Debatte zur Benennung des Phänomens beleuchtet. Zur Bezeichnung des Eingriffs werden unterschiedliche Termini herangezogen, die von ,Beschneidung' über , weibliche Beschneidung' bis hin $\mathrm{zu}$,weibliche Genitalverstümmelung ' oder englischen Begrifflichkeiten wie ,Female Genital Cutting' (FGC) oder ,Female Genital Mutilation“ (FGM) variieren. Zudem existieren einheimische Bezeichnungen, die sich je nach Land und Sprache voneinander unterscheiden, zumeist aber keinerlei Bezug zu dem Eingriff aufweisen.

Der Begriff ,Female Genital Mutilation“, zu Deutsch ,weibliche Genitalverstümmelung', wird bis heute kontrovers diskutiert. Geprägt wurde der Begriff von afrikanischen, selbst von der Praktik betroffenen Aktivistinnen mit dem Ziel, weltweit darauf hinzuweisen, dass es sich um einen schwerwiegenden und nicht rückgängig zu machenden Eingriff handelt, der nicht mit der männlichen Beschneidung vergleichbar oder gar gleich zu setzen ist (Richter/Schnüll 2003, S. 16). In den 90er Jahren des vergangenen Jahrhunderts hat sich dieser Begriff auch im Inter-African-Comittee (IAC) und bei den Vereinten Nationen durchgesetzt (ebd.). Auf politischer Ebene war dieser Begriff lange Zeit dominant, um die Tragweite des Eingriffs deutlich zu benennen. Mittlerweile ist zu beobachten, dass sich der Begriff ,Female Genital Cutting' (FGC) oder im Deutschen, weibliche Genitalbeschneidung' sowohl in Publikationen als auch in politische Diskussionen wieder stärker durchsetzt (Leye 2008, S. 22). Dies hängt damit zusammen, dass sich viele Betroffene von dem Bild der, verstümmelten Frau' distanzieren wollen, da sie sich von diesem stark stigmatisiert und auf ihre Genitalien reduziert fühlen. Diese Frauen lehnen den Begriff ,Verstümmelung' ab und wollen auch nicht als ,verstümmelt' bezeichnet werden. Betroffene, die diese Meinung vertreten, identifizieren sich stärker mit dem Begriff ,Beschneidung' oder der Tat- 
sache ,beschnitten zu sein' und halten an dieser eher neutralen, weniger wertenden Form fest (Dirie 2003, S. 102).

In den einzelnen Ländern finden Begriffe Verwendung, die häufig nichts mit dem Eingriff zu tun haben. Nicht selten sind diese positiv konnotiert, wie beispielsweise der eritreische Begriff ,mekinschab', der ,rein' bedeutet (Asefaw 2008, S.12). Auf Somalisch gibt es zwei Bezeichnungen: ,Gudniin` heißt ,Beschneidung', der zweite Begriff ,Halaaleyn` bedeutet, wie auch im Eritreischen, ,bereinigen, rein machen'. Mädchen sind erst ,rein', wenn die Klitoris entfernt wurde: Mit Klitoris würden sie in die Hölle kommen, so die damit zusammenhängende Begründung. In der vorliegenden Arbeit wird der Begriff ,Genitalbeschneidung' verwendet, da die im Rahmen der Forschung durchgeführten Interviews mit somalischen Frauen und Männer gezeigt haben, dass die Mehrheit der Interviewten selbst diesen Begriff wählt, sich selbst demnach nicht als, verstümmelt' betrachtet. Begriffe wie ,Female Genital Mutilation' (FGM) oder, weibliche Genitalverstümmelung' werden somit nur im Rahmen von Zitaten Verwendung finden.

Die steigende Anzahl der weltweit sich in Migration befindenden genital beschnittenen Mädchen und Frauen sorgt dafür, dass es sich auch in Europa um ein gesellschaftlich relevantes Phänomen handelt, dem sich bereits einige Organisationen angenommen haben. Diese versuchen, eingewanderte Mädchen vor dem Eingriff zu schützen und betroffene Frauen im Umgang mit den Konsequenzen des Eingriffs zu unterstützen. Die im Rahmen meiner Diplomarbeit (2006) durchgeführte quantitative Erhebung zur Beratungssituation beschnittener und von Beschneidung bedrohter Mädchen und Frauen in Deutschland und Frankreich hat jedoch ergeben, dass Diskrepanzen zwischen der Arbeit der Organisationen und den Bedürfnissen betroffener und bedrohter Mädchen und Frauen bestehen (vgl. Ihring 2006). Diese kommen besonders darin zum Ausdruck, dass zwar etliche Angebote für Betroffene und Bedrohte existieren, die aber häufig nicht von der Zielgruppe in Anspruch genommen werden (vgl. ebd.). Dieses Ergebnis war ausschlaggebend für die Idee, im Rahmen der vorliegenden Dissertation erneut die Perspektive von Fachkräften, die in Einrichtungen und/oder Kliniken gegen weibliche Genitalbeschneidung arbeiten, in den Vordergrund zu stellen, um zu eruieren, wie sich mittlerweile die Herangehensweisen gestalten.

Um ein möglichst breites Spektrum an Herangehensweisen zu erfassen, wurde mit dem teilstandardisierten Fragebogen ein quantitatives Erhebungsinstrument zur Durchführung dieser Untersuchung gewählt, der an Einrichtungen und Kliniken in Deutschland, England, Italien und der Schweiz verschickt wurde. Grundlage dieser Untersuchung bildete die Fragestellung, wie die Arbeit gegen weibliche Genitalbeschneidung künftig so gestaltet werden kann, dass bedrohte Mädchen nachhaltig geschützt werden und Angebote betroffene Frauen in ihren Bedürfnissen erreichen. Unter Zuhilfenahme des teilstandardisierten Fragebogens, der an alle bestehenden Beratungsstellen 
und/oder Kliniken versendet wurde, konnten Einblicke gewonnen werden, in welcher Form sich die Arbeit gegen Mädchenbeschneidung in den jeweiligen Untersuchungsländern institutionalisiert hat und ob sie von der Zielgruppe in Anspruch genommen werden. Der teilstandardisierte Fragebogen bestand aus Fragen zu den konkreten Angeboten für genital beschnittene Frauen, sowie aus Fragen zu Präventionsangeboten und der Vernetzung der befragten Organisationen mit Fachpersonal, das im Umgang mit gynäkologischen oder psychologischen Konsequenzen informiert ist. Der europäische Vergleich erschien dabei besonders interessant, da die untersuchten Länder Deutschland, England, Italien und die Schweiz sich aufgrund ihrer Migrationspolitik, aber auch der Zahl an von Mädchenbeschneidung betroffenen Migrantinnen im Land, in ihrem Umgang mit dem Phänomen unterscheiden.

Die Auswahl der Einwanderungsländer hing eng mit der Wahl der zu befragenden Bevölkerungsgruppe zusammen. Da die Bevölkerungsgruppe der Somalis in dieser Arbeit im Zentrum steht, wurden Einwanderungsländer gewählt, in denen Menschen aus Somalia leben. Anfangs sollten die Untersuchungen neben Deutschland in England, Italien und den Niederlanden durchgeführt werden, weil in den drei Letztgenannten eine hohe Zahl somalischer Migrant_innen lebt. Neben England, mit schätzungsweise 70000 bis 100000 Somalis, handelt es sich auch in den Niederlanden um einen hohen Anteil somalischer Einwanderer_innen, der auf circa 20000 Somalier_innen geschätzt wird (Edwards 2007). Nachdem sich jedoch abzeichnete, dass sich Beratungsstellen aus den Niederlanden nicht an der Untersuchung beteiligen, wurde die Länderauswahl modifiziert und die Niederlande durch die Schweiz ersetzt. Laut den statistischen Angaben von 2012 leben circa 8000 Somalier_innen in der Schweiz, die ursprünglich alle als Asylbewerber_innen gekommen sind (Abdeleli 2013). Im Vergleich zu Italien oder England handelt es sich um eine geringe Anzahl, die der Situation in Deutschland ähnelt. In Deutschland leben laut Statistischem Bundesamt circa 10000 Somalis. Da viele Somalis (noch) keinen anerkannten Aufenthaltsstatus im jeweiligen Einwanderungsland haben oder illegal in den Ländern leben, beruhen die Zahlen teilweise auf Schätzungen. Auch in Italien lebt eine große Zahl somalischer Flüchtlinge, die meist von Libyen nach Lampedusa geflohen sind und darauf hoffen, in Europa ein besseres Leben vorzufinden.

Hinter dieser Auswahl stand die Vermutung, dass die Länder, in denen eine hohe Anzahl an Flüchtlingen aus Somalia leben, besser mit deren Problemen vertraut sind. So kann angenommen werden, dass das Problem weiblicher Genitalbeschneidung aufgrund der Vielzahl an Somalis in Italien, den Niederlanden und England bereits diskutiert und versucht wird einen Weg zu finden, der Betroffenen im Umgang mit den Folgen der Praktik hilft. Die Tatsache, dass in allen drei Ländern bereits ein gesetzliches Verbot gegen Genitalbeschneidung besteht, zeigt, dass sich auf politischer Ebene schon sehr intensiv mit der Praktik auseinandergesetzt wurde. Insgesamt zielte 
dieser europäische Vergleich mithilfe teilstandardisierten Fragebogenerhebung darauf ab, einen Einblick vom Umgang anderer Länder mit dieser Problematik zu bekommen, so dass von den unterschiedlichen Ansätzen auch die Arbeit in Deutschland profitieren kann.

Der zweite Fokus dieser Forschungsarbeit lag darauf, die Perspektive beschnittener somalischer Frauen und Männer zu erfassen. Die subjektive Perspektive von betroffenen Migrant_innen, die im Laufe ihres Lebens in die genannten Einwanderungsländer immigriert sind, wurde mithilfe qualitativer Interviews erfasst. Die qualitativen Interviews mit den weiblichen Interviewpartnern basierten auf der Frage, welche Handlungsstrategien beschnittene Frauen entwickelt haben, um mit den Veränderungen umzugehen, die mit dem Verlassen ihres Heimatlandes im Hinblick auf Genitalbeschneidung verbunden sind. Die Perspektive der somalischen Frauen wurde sowohl mit biographisch-narrativen als auch mit problemzentrierten Interviews beleuchtet. Die Kombination dieser beiden Interviewmethoden war sinnvoll, da die Frauen einen Teil ihres Lebens in Somalia verbracht und dort ihre eigene Beschneidung erlebt haben. Zur Rekonstruktion ihrer heutigen Sicht auf weibliche Genitalbeschneidung erschien diese Lebensphase bedeutsam, da die Rolle, die Beschneidung in der Sozialisation der Frauen einnimmt, ein besseres Verständnis des Phänomens ermöglicht. Des Weiteren ist der Blick auf das Leben vor ihrer Migration in ein europäisches Land interessant, um die durch diesen Schritt erfolgten möglichen Veränderungen im Leben der Frauen zu erfassen. In Bezug auf beschnittene somalische Frauen kann dieser Schritt bedeuten, in vielerlei Hinsicht nicht länger der gesellschaftlichen ,Norm` anzugehören. Welche Handlungsstrategien beschnittene Frauen entwickelt haben, um mit diesem Wandel umzugehen, wurde mithilfe des problemzentrierten Interviewteils erfasst.

Die Interviews mit den Männern dagegen bestanden ausschließlich aus einem problemzentrierten Interview und basierten auf der Frage, welche Sichtweisen Männer auf weibliche Genitalbeschneidung haben. Der Fokus dieser Untersuchung lag ausschließlich auf deren Perspektive in Bezug auf das Phänomen, weshalb die Wahl auf das problemzentrierte Interview als alleinige Methode gefallen ist. Die ergänzenden Interviews zur Sichtweise männlicher Somalis im Kontext von weiblicher Genitalbeschneidung waren nicht nur von Interesse, da diese Perspektive wenig erforscht ist, sondern gleichzeitig von Bedeutung, weil Männer bezüglich des zu untersuchenden Phänomens eine wichtige Rolle einnehmen, da sie als Mitglieder einer Genitalbeschneidung praktizierenden Gesellschaft dazu beitragen, die Praktik aufrechtzuerhalten. Die zentralen Aspekte dieser Interviews wurden zusammengefasst und in einem gesonderten Kapitel dargestellt.

Die Wahl, die Perspektive somalischer Migrant_innen zu analysieren, wurde aus mehreren Gründen getroffen. Zum einen handelt es sich bei der Bevölkerungsgruppe der Somalis um eine weitgehend einheitliche Bevölke- 
rungsgruppe, deren Angehörige dieselbe Sprache sprechen und sich auch im Glauben nicht unterscheiden (Vgl. Birnbaum 2007, S. 293; vgl. Schicho 2004; vgl. Bäßler/Wallach/Ender 2006). Aufgrund ihres undurchsichtigen Klansystems herrscht jedoch seit Ende der Diktaturherrschaft durch Muhammad Siad Barré 1991 Bürgerkrieg (Birnbaum 2007, S. 293). Diese politische Situation trägt stark dazu bei, dass die Zahl der weltweit sich in Migration befindenden Somalis hoch ist (vgl. Birnbaum 2007, S. 294; Lewis 2008). Nach Angaben der Vereinten Nationen gehören die Somalis mit 1,1 Millionen Menschen weltweit zur Spitze unter den Flüchtlingen (UNO 2013). In England, Italien und der Niederlande, sowie in den USA und Kanada gibt es mittlerweile eine große Anzahl somalischer Migrant innen (UNHCR 2012). Zum anderen handelt es sich um eine Bevölkerungsgruppe, in der nahezu alle Frauen beschnitten sind, nach Schätzungen der Weltgesundheitsorganisation 98 Prozent (UNICEF 2008, S. 208).Des Weiteren sind sie von der schwersten Form weiblicher Beschneidung, der Infibulation, betroffen, was die Wahrscheinlichkeit, von psychischen und/oder medizinischen Konsequenzen betroffen zu sein, deutlich erhöht. Ein weiterer Aspekt, der die Wahl der somalischen Bevölkerungsgruppe begünstigte, ist mein eigener somalischer Hintergrund, der nicht nur ein grundsätzliches Interesse an der Bevölkerungsgruppe der Somalis mit sich bringt, sondern auch den Zugang zu dieser Bevölkerungsgruppe erleichtert hat.

Die Forschung wird durch unterschiedliche theoretische Perspektiven gerahmt, die nicht nur bei der Rekonstruktion der Interviews eine wichtige Rolle spielen, sondern auch zum Verständnis des Phänomens in seinen vielen Facetten beitragen. Die Grundlage bildet dabei die sozialkonstruktivistische Annahme, dass „Gesellschaftsordnung weder biologisch gegeben noch von irgendwelchen biologischen Gegebenheiten ableitbar" (Berger/Luckmann 1969, S. 55) ist. Vielmehr handelt es sich um eine Produktion der Menschen, die sie ,mit der ganzen Fülle ihrer sozio-kulturellen und psychologischen Gebilde" (ebd., S. 54) ausstatten. Aus sozialkonstruktivistischer Sicht werden soziale Wirklichkeit und die darin vorkommenden Phänomene von ihren Betrachter innen hergestellt. Die Vorstellung einer durch Menschen konstruierte Wirklichkeit und den daraus resultierenden individuellen Wahrnehmungen führt dazu, dass das Phänomen weiblicher Genitalbeschneidung aus unterschiedlichen Richtungen heraus betrachtet werden muss. Ein wesentlicher Teil dieser Arbeit ist daher die Darstellung unterschiedlicher theoretischer Perspektiven, die im Kontext des zu untersuchenden Phänomens von Bedeutung sind.

Die aus den Postkolonialen Theorien hervorgegangenen Erkenntnisse spielen angesichts der Tatsache, dass mit der Wahl, somalische Frauen und Männer zu interviewen, eine Bevölkerungsgruppe ausgewählt wurde, die auf eine Kolonialgeschichte zurückblickt, eine bedeutende Rolle. Die Folgen kolonialer Besetzung wirken sich bis heute nicht nur auf die kolonisierten Bevölke- 
rungsgruppen aus, sie haben globale Konsequenzen und finden sich unterschiedlichen Facetten bis heute wieder (vgl. Castro Varela/Dhawan 2005, S. 24). Die aus den Postkolonialen Studien hervorgegangene Kritische Weißseinsforschung richtet den Blick auf die weiße $e^{4}$ Mehrheitsgesellschaft und deckt die Konsequenzen auf, die sich aus diesem, häufig unmarkierten Blickwinkel für Schwarze/'People of Color ${ }^{65}$ ergeben (Arndt 2006, S.13). Die Geschichte des europäischen Feminismus und der daraus resultierenden Genderforschung, ist im Kontext von weiblicher Genitalbeschneidung ebenfalls von Bedeutung. In diesem Teil steht die Konstruktion von Geschlecht und den damit verbundenen Zuschreibungen und deren gesellschaftlichen Auswirkungen im Zentrum des Interesses. Damit eng verbunden ist die Konstruktion von Normen, die im Zusammenhang mit dem zu untersuchenden Phänomen von Interesse ist, da es sich bei weiblicher Genitalbeschneidung um eine gesellschaftlich etablierte Norm handelt und Normen eng mit gesellschaftlichen Macht- und Herrschaftsverhältnissen verbunden sind (Vgl. Engel/Schuster 2007; Foucault 1991). Die intersektionale Perspektive betrachtet schließlich die Wechselwirkungen zwischen den einzelnen Kategorien wie Geschlecht, Klasse, ,Rasse“ und Körper, die zum Erhalt sozialer Ungleichheit beitragen und nicht getrennt voneinander gedacht werden können (Vgl. Walgenbach 2012, S. 81; Winker/Degele 2010). Im Kontext beschnittener Migrantinnen somalischer Herkunft ist dies eine wichtige Perspektive, die auch bei der Auswertung der Interviews als Analyseinstrument herangezogen wird.

Ebenfalls Teil des theoretischen Rahmens ist die Debatte um ,Kultur', die im Zusammenhang mit weiblicher Genitalbeschneidung grundsätzlich als Argument zur Rechtfertigung der Praktik genannt wird. Die Bedeutung von ,Kultur' als Bezugs- und Orientierungsrahmen ist im Kontext von Migration entscheidend und wird im Hinblick auf somalische Migrant_innen betrachtet.

\section{Aufbau der Arbeit}

Das erste Kapitel beschäftigt sich mit dem Phänomen weiblicher Genitalbeschneidung. Neben der Definition des Eingriffs wird der Blick auf dessen medizinische und psychische Konsequenzen gerichtet. Teil dieses Kapitels ist auch die politische und soziale Lage Somalias sowie die besondere Situation

4 Die Schreibweise kursiv zur Benennung von weiß und groß für Schwarz wird, wie in der Kritischen Weißseinsforschung üblich, in dieser Form übernommen, um zu kennzeichnen, dass es nicht im biologistischen Sinne gemeint, sondern als Konstruktion sichtbar zu machen ist. (Vgl. Arndt/Eggers/Kilomba/Piesche (Hg.) 2005).

5 ,People of Color' ist ein Begriff, der der Black Power Bewegung der USA entsprungen ist und sich auf alle Menschen bezieht, die durch die weiße Dominanzkultur unterdrückt oder abgewertet werden (Vgl. Eggers 2005). 
der Frauen im Land. Da sich der Hauptteil der Dissertation mit Frauen und Männern beschäftigt, die ursprünglich in Somalia geboren und aufgewachsen sind, ist der Blick auf die Situation in ihrem Heimatland von Bedeutung, um die Interviewaussagen in allen Facetten verständlich zu machen. Abschließend wird auf die gesetzliche Lage bezüglich weiblicher Genitalbeschneidung in afrikanischen Staaten sowie auf die gesetzlichen Regelungen der im Kontext dieser Arbeit fokussierten Einwanderungsländer Deutschland, England, Italien und die Schweiz eingegangen.

Im Anschluss daran steht im zweiten Kapitel die Migrationspolitik Deutschlands, Englands, Italiens und der Schweiz im Vordergrund. Die Migrationspolitik der einzelnen Länder, die besonders darin besteht illegale Einwanderung zu unterbinden und Einwanderung nur für ausgewählte Bevölkerungsgruppen möglich zu machen, hat nachhaltige Auswirkungen auf die im Kontext dieser Arbeit gewählte Bevölkerungsgruppe der Somalis, was in den Ausführungen in diesem Kapitel deutlich wird.

Thematisch daran anschließend beginnt das dritte Kapitel mit den Postkolonialen Studien, die eng mit der Kritischen Weißseinsforschung einhergehen und als theoretische Perspektive im Kontext weiblicher Genitalbeschneidung dienlich sind. Neben diesen Perspektiven, wird auf die Entwicklung der Frauenbewegung und die feministischen Theorien eingegangen. Im Anschluss daran wird die intersektionale Perspektive dargestellt und abschlieBend noch die Debatte um ,Kultur'. Die theoretische Rahmung bietet nicht nur Möglichkeiten, das Phänomen weiblicher Genitalbeschneidung differenziert zu betrachten, die Erkenntnisse der Theorien spiegeln sich auch in den Interviewaussagen der somalischen Frauen und Männer wieder.

Im vierten Kapitel wird das methodische Vorgehen und Forschungsdesign der im Rahmen dieser Arbeit durchgeführten Untersuchungen vorgestellt. In einem ersten Teil wird auf die Methodik der quantitativen Untersuchung und deren Durchführung eingegangen. In einem zweiten Teil werden dann die qualitativen Interviewmethoden, die Interviewdurchführung, sowie deren Analyse vorgestellt.

Die Präsentation der Ergebnisse der quantitativen Untersuchung erfolgt in Kapitel fünf. Die Ergebnisse werden nach jeweiligem Einwanderungsland präsentiert und abschließend diskutiert. In Kapitel sechs erfolgt die Analyse der qualitativen Interviews mit den somalischen Frauen. In Form von Fallanalysen werden die Interviews präsentiert. Im Anschluss daran sind die zentralen Ergebnisse der Fallanalysen in Kapitel sieben festgehalten.

Kapitel acht besteht aus der Zusammenfassung der zentralen Aussagen aus den qualitativen Interviews mit den männlichen Somalis. Diese werden nach der Darstellung der Aussagen noch abschließend diskutiert werden.

In Kapitel neun werden die Ergebnisse der jeweiligen Untersuchungen mit den Erkenntnissen aus den verschiedenen Theorien verbunden und diskutiert. Daraus ergeben sich die nachfolgend dargestellten Konsequenzen für Politik 
und Beratung, die Teil des zehnten Kapitels sind. Das Fazit und der Ausblick bilden in Kapitel elf den Abschluss dieser Dissertation. 


\section{Theoretischer Rahmen}

\section{Weibliche Genitalbeschneidung}

Bei weiblicher Genitalbeschneidung handelt es sich um eine sehr alte Praktik, von der angenommen wird, dass sie ihren Ursprung in Ägypten hat (Hulverscheidt 2002, S. 25). Neben Wandmalereien weisen auch späteren Schriften der Antike auf, dass die Beschneidung des weiblichen Genitales in Ägypten durchgeführt wurde. Es bleibt zwar offen, welche Teile des Genitales beschnitten wurden und ob sie nur an Frauen aus bestimmten Bevölkerungsgruppen vorgenommen wurde, dennoch finden sie Erwähnung (ebd., S. 26). Im Mittelalter gewann die arabische Medizin neben der des Abendlandes an Bedeutung und die Beschneidung der Klitoris wurde als notwendiger operativer Eingriff angesehen, wenn sie ein krankhaftes Wachstum zeige (ebd., S. 28). Nach und nach entwickelte sich auch - und gerade - in Europa eine Medizin, die die Beschneidung des weiblichen Genitales unter gewissen Umständen als notwendigen Eingriff ansah. Ihren Höhepunkt fanden die Verstümmelungen des weiblichen Genitales in der zweiten Hälfte des 19. bis Mitte des 20. Jahrhunderts. Diese Zeit wurde von der Vorstellung geprägt, Masturbation sei ein krankhaftes, zu behandelndes Phänomen. Es entstand eine „wachsende Masturbationshysterie [...], die Ärzte mit der Macht ausstattete, über die Sexualität der Gesellschaft zu bestimmen“ (Lightfoot-Klein 2003, S. 24). Es wurden zahlreiche Gründe gefunden, die die Beschneidung des weiblichen Genitales medizinisch notwendig machten. So wurde neben der Masturbation auch „lesbische Neigungen“, „weibliche Geisteszustände wie Hypersexualität, Hysterie und Nervosität“ oder „Epilepsie, Katatonie, Melancholie und Kleptomanie“" (ebd., S. 24) als Begründungen für die Entfernung der Klitoris angeführt. Dieser Maßnahme liegt die Sichtweise zugrunde, der weibliche Geschlechtstrieb wäre in den Geschlechtsorganen selbst lokalisiert (Hulverscheidt 2002, S.40). Es bestand die Vorstellung, dass defekte oder hypertrophe Geschlechtsorgane für Masturbation und Nymphomanie verantwortlich wären. So wurde der Grund für Nymphomanie und Hysterie erst der Gebärmutter, dann den Eierstöcken und am Ende der Klitoris zugeschrieben (Lightfoot-Klein 2003, S. 26). Fortan sollten Mediziner Masturbation, Nymphomanie und genannte Geisteszustände durch Klitoridektomien behandeln. Diese Vorstellungen hielten sich bis ins 20. Jahrhundert. So empfahl die katholische Kirche „noch bis 1940 die Verätzung oder Amputation der Klitoris gegen das Laster des Lesbiertums“ (Lightfoot-Klein 
2003, S. 34). Auch in medizinischen Lehrbüchern wurde Masturbation bis in die 70er Jahre des 20. Jahrhunderts als Krankheit benannt (ebd.).

Der kurze Rückblick auf die Geschichte zeigt, dass es sich bei der Beschneidung der Genitalien keinesfalls um ein selten vorkommendes oder nur bestimmte Bevölkerungsgruppen oder -schichten betreffendes Phänomen handelt. Der operative Eingriff an den Genitalien ist ein weltweit verbreitetes Phänomen, das seit Jahrtausenden praktiziert wird.

\subsection{Definition und Formen weiblicher Genitalbeschneidung}

Die Weltgesundheitsorganisation (WHO) hat Genitalbeschneidung definiert und in verschiedene Typen unterteilt. Sie definiert weibliche Genitalbeschneidung folgendermaßen:

„Female genital mutilation (FGM) comprises all procedures that involve partial or total removal of the external female genitalia, or other injury to the female genital organs for non-medical reason." (WHO 2014)

Nach Schätzungen der WHO sind weltweit circa 140 Million Mädchen und Frauen betroffen, weitere drei Millionen sind von der Praktik bedroht. Die WHO unterscheidet vier Typen (ebd.) der genitalen Beschneidung bei Frauen:

Typ I (Klitoriedektomie): die partiellen oder vollständigen Entfernung der Klitoris

Typ II (Exzision): die teilweise oder vollständige Entfernung der Klitoris sowie die teilweise oder vollständige Entfernung der inneren Labien.

Typ III (Infibulation): die Entfernung der Klitoris, sowie die der inneren Labien und der inneren Schichten der äußeren Labien. Die Innenseiten derselben werden anschließend miteinander vernäht. Zurück verbleibt den Mädchen und Frauen eine kleine Öffnung, durch die Urin und Menstruationsblut austreten kann.

Typ IV: darunter fallen alle weiteren Praktiken, die die inneren oder äußeren Genitalien verletzen oder verändern ohne medizinische Indikation (z. B. Piercen der Klitorishaut oder Dehnen der Labien).

Verbreitet ist die Praktik besonders in afrikanischen Ländern und zieht sich wie ein Gürtel von West- nach Ostafrika, im Norden praktizieren ebenfalls manche Bevölkerungsgruppen den Eingriff, im Süden Afrikas hingegen nicht. Doch auch in arabisch geprägten Ländern in verschiedenen Regionen 
Asiens und des Mittleren Ostens werden Mädchen beschnitten (Richter/Schnüll 2003, S. 59). Es handelt sich um eine Praktik, die an bestimmte Bevölkerungsgruppen gebunden ist, daher ist es auch nicht möglich, sie ganzen Ländern zuzuordnen. So ist der Eingriff beispielsweise unter irakischen Kurden verbreitet, einer Bevölkerungsgruppe, mit der die Praktik bislang weniger in Verbindung gebracht wurde (vgl. Wadi e.V. 2012). In Somalia sind 98 Prozent aller Mädchen und Frauen beschnitten, die Mehrheit infibuliert (UNICEF 2008, S. 208). Durch zunehmende Migration ist Genitalbeschneidung auch in Europa oder Nordamerika zunehmend verbreitet.

\subsection{Mögliche Konsequenzen weiblicher Beschneidung}

Da somalische Mädchen und Frauen im Zentrum dieser Arbeit stehen und diese überwiegend von Typ III, der Infibulation, der schwersten Form betroffen sind (vgl. Terre des Femmes 2003; UNICEF 2008), wird dieser Beschneidungstyp im Folgenden im Vordergrund stehen. Dennoch soll an dieser Stelle betont werden, dass nicht jede infibulierte Frau zwingend von dem gesamten Ausmaß der Folgen betroffen sein muss. Die Wahrscheinlichkeit, dass infibulierte Frauen jedoch von manchen Folgen häufiger betroffen sind als Frauen, die auf weniger schwerwiegende Weise beschnitten wurden, ist höher. Die physischen Konsequenzen des Eingriffs sind mittlerweile detailliert durch Studien belegt, während umfassende Studien zu den psychischen Konsequenzen und den Auswirkungen auf das sexuelle Erleben und Empfinden betroffener Frauen fehlen.

Die akuten, also unmittelbar während des Eingriffs auftretenden Konsequenzen, sind vielschichtig. Beim weiblichen Genital handelt es sich um ein sehr nervenreiches und gut durchblutetes Organ. Da der Eingriff in der Regel ohne Anästhesie durchgeführt wird, leiden die Mädchen und Frauen unterdessen unter enormem Schmerzen und gegebenenfalls unter hohem Blutverlust. Der hohe Blutverlust kann Schockzustände oder Anämien auslösen und im schlimmsten Fall tödlich enden (Bauer/Hulverscheidt 2003, S. 67ff.). Da der Eingriff häufig von Beschneiderinnen durchgeführt wird, die über keine medizinische Ausbildung verfügen, kommt es vielfach vor, dass auch umliegende Organe verletzt werden (wie beispielsweise die Analmuskulatur und/oder die Harnröhre), was nicht selten eine Inkontinenz zur Folge hat (vgl. Bundesärztekammer 2013). Wenn sich die Mädchen oder Frauen stark zur Wehr setzen, sind auch Knochenbrüche, ausgekugelte Schultergelenke und/oder Zungenbisse keine Seltenheit. Infektionen, wie beispielsweise Sepsis, Wundbrand, Wundstarrkrampf, Hepatitis oder HIV, können ebenfalls auftreten, da der Eingriff oftmals unter unsterilen Bedingungen stattfindet 
(Terre des Femmes 2005; Bundesärztekammer 2013). Akuter Harnverhalt aus Angst vor weiteren Schmerzen tritt häufig unmittelbar nach dem Eingriff auf.

Unter chronischen Konsequenzen werden all die Auswirkungen zusammengefasst, die die Frauen langfristig begleiten können. Studien zeigen, dass infibulierte Frauen häufiger von chronischen Konsequenzen betroffen sind als Frauen die auf eine weniger invasive Form beschnitten wurden (vgl. Toubia 1994). Von chronischen Infektionen sind die Harnorgane (zum Beispiel chronische Harnwegsinfekte, Infekte des kleinen Beckens und der Nieren) und auch die Scheide, die Gebärmutter und der Eileiter betroffen. Chronisch entzündete Eileiter verkleben und führen zur Sterilität der Betroffenen (Bauer/Hulverscheidt 2003, S. 67ff.). Eine weitere Folge, die vor allem infibulierte Mädchen und Frauen betrifft, ist der so genannte Hämatokolpos. Dies bedeutet, dass die verbliebene vaginale Öffnung nicht groß genug ist, um das Abfließen des Menstruationsblutes und Urins ausreichend zu gewährleisten, Blut und Urin stauen sich und führen zum Anschwellen des Bauches. Dies kann hoch bis zur Gebärmutter und dem Eileiter reichen und dort Infektionen auslösen, die ebenfalls Infertilität zur Folge haben können (vgl. Terre des Femmes 2005; Bundesärztekammer 2013). Weitere Folgen sind starke Menstruationsbeschwerden, Abszessbildung an der Narbe, starker Wuchs von Narbengewebe (Keloidbildungen), das zur Verengung der Harnwege oder Vagina führt und zu Schmerzen beim Geschlechtsverkehr und Harnverhalt (ebd.). Eine häufige Komplikation ist die so genannte Fistelbildung, die aufgrund der chronischen Infektionen zustande kommt. Fisteln sind ,häutig ausgekleidete Verbindung zwischen zwei Hohlorganen, also zwischen Scheide und der Blase oder der Scheide und dem Mastdarm." (Bauer/Hulverscheidt 2003, S. 69). Eine Fistel hat zur Folge, dass Urin oder Stuhl durch die Scheide abgehen und die Frau keinerlei Kontrolle mehr über diesen Vorgang hat. Die daraus entstehende Inkontinenz hat für Betroffene auch soziale Folgen: die Frauen werden ausgegrenzt, da sie nach Exkrementen riechen (ebd.).

Bei infibulierten Frauen ist die vaginale gynäkologische Untersuchung oft nicht problemlos möglich, da die verbliebene Öffnung zu klein ist, um eine vaginale Untersuchung durchzuführen. Auch für die Geburt eines Kindes kann die Verstümmelung schwere Folgen haben. Das starre Narbengewebe ist nicht mehr flexibel genug um der Dehnung nachzugeben, die beim Austritt des Kindskopfes nötig ist (Bauer/Hulverscheidt 2003, S. 76; Bundesärztekammer 2013). So entstehen Risse im Gewebe, die wichtige Nervenbahnen verletzen können was wiederum zu Infektionen führen kann. Des Weiteren kann sich das Gewebe während der Geburt nicht genug weiten, wodurch es zu Verzögerungen bis hin zum kompletten Stillstand des Geburtsvorganges kommen kann (Bundesärztekammer 2013). Dies kann sowohl für die Frau als auch für das Neugeborene tödliche Folgen haben (vgl. Toubia 1994). Im Rahmen ihrer Forschungen im Sudan war die Sozialpsychologin Lightfoot- 
Klein bei einigen Geburten sudanesischer, infibulierter Frauen zugegen und berichtet, keine infibulierte Frau erlebt zu haben, die spontan ohne Defibulation (operatives Öffnen der Narbe) entbinden konnte (Lightfoot-Klein 1992, S. 79); ein Eingriff, der laut Lightfoot-Klein wieder mit teilweise starken Blutungen und Schmerzen verbunden sei und häufig mit Verletzung der Harnwege oder Teilen des Darms einhergehe (ebd.). Nach der Geburt erfolgt meist die Reinfibulation, das erneute Zunähen der Scheide bis auf die ursprüngliche Öffnung.

Eine weitere Folge sind Zysten, die aufgrund der Verzögerung des Geburtsverlaufes entstehen können (Bundesärztekammer 2013). Die starke und viel länger andauernde Dehnung des Gewebes und der Muskulatur um die Gebärmutter herum, kann zu einem Gebärmuttervorfall führen (ebd.). Dies geschieht vor allem bei mehreren, kurz aufeinander folgenden Geburten. Des Weiteren ist laut WHO davon auszugehen, dass die Beschneidung, besonders die Infibulation von Frauen, eine erhöhte Mütter- und Kindersterblichkeit mit sich bringt (vgl. WHO).

Bevor auf den Punkt eingegangen wird, welche Auswirkungen sich aus dem Eingriff möglicherweise für die weibliche Sexualität ergeben, soll angemerkt werden, dass es sich beim Thema Sexualität um ein Thema handelt, das je nach kulturellem Hintergrund anders ge- und erlebt wird (Hulverscheidt/Ahlers/Ihring 2009, S. 23). Das bedeutet, dass die sexuelle Erregbarkeit einer Frau nicht zwingend mit der Klitoris verbunden sein muss. Frauen, deren Klitoris ganz oder teilweise entfernt wurde, die Orgasmusfähigkeit abzusprechen, wäre anmaßend, da sexuelle Erregung und Befriedigung nicht allein über die Genitalien, sondern über das Gehirn gesteuert werden (ebd.). Die Vorstellung sexuelle Erregbarkeit und Empfindsamkeit würde vornehmlich von der Stimulation der Klitoris abhängen, ist eine sehr , westlich ${ }^{6}$ geprägte Vorstellung. Bei infibulierten kommt hinzu, dass nicht allein die Klitoris beschnitten wurde, sie wurden nach dem Eingriff zusätzlich vernäht. Die daraus entstandene Öffnung oft sehr klein ist, vaginaler Geschlechtsverkehr

Im Kontext dieser Arbeit wird unter, Westen' die Bedeutung verstanden, die Stuart Hall (1994) in seinem Text „The West and the Rest“ detailliert erläutert. Hall zeigt darin, dass hinter der Bezeichnung, westliche'Gesellschaft nicht die geographische Verortung bestimmter Bevölkerungsgruppen steht. Vielmehr handelt es sich um ein aus der Geschichte hervorgegangenes Konstrukt (Hall 1994, S. 186), das zur Formung der Identität und Zusammengehörigkeit Europas gedient hat. In Abgrenzung zu den ,Anderen“ ist der ,Westen “ ein Vergleichs- und Erklärungsmodell, das nicht für sich selbst stehen kann, sondern nur in Abgrenzung zum Rest funktioniert (ebd., S.186). Das Konstrukt des ,Westens ‘ ermöglicht es, Gesellschaftssysteme miteinander zu vergleichen und bestimmte Bevölkerungsgruppen als ,westlich' zu charakterisieren und mit Zuschreibungen wie ,modern', ,entwickelt', ,wünschenswert‘ (ebd.) zu versehen. Im Unterschied zum ,Westen` stehen die ,nichtwestlichen' Gesellschaften, die in diesem Vergleich als ,unmodern' oder ,unentwickelt' etikettiert werden. Laut Hall handelt es sich um ein Modell, das bestimmtes Wissen über eine Gruppe hervorbringt und dieser mit einer gewisse Haltung gegenüber steht. Aus Halls Sicht gleicht die Vorstellung vom ,Westen` einer Ideologie (ebd.). 
demnach nicht ohne Weiteres möglich (Terre des Femmes 2005). Bei infibulierten Frauen ist daher die Chance größer, Schwierigkeiten beim vaginalen Geschlechtsverkehr zu haben. Dennoch darf nicht generell ein unerfülltes Sexualleben unterstellt werden, da sexuelles Empfinden und sexuelle Freuden auch auf andere Weise erlebt werden können und weder eine vollständige Klitoris noch vaginaler Geschlechtsverkehr dafür grundsätzlich nötig sind.

Die zu kleine Öffnung infibulierter Frauen erschwert die vaginale Penetration und sorgt insbesondere beim ersten Geschlechtsverkehr für große Schmerzen (Bauer/Hulverscheidt 2003, S. 75). Nicht selten ist der vaginale Verkehr daher nicht ohne Hilfsmittel möglich, da das männlichen Glied allein zum Weiten des starren Narbengewebes nicht ausreicht (ebd., S. 74). Daher werden häufig scharfe Gegenstände benutzt, um die winzige Öffnung ein wenig weiter aufzuschneiden (Asefaw 2008, S. 62). Das gewaltsame Eindringen führt $\mathrm{zu}$ weiteren Schmerzen, Narbenrissen, Infektionen und Keloidbildungen (ebd., S. 69). Frauen, die auf gewaltsame Weise geöffnet werden müssen, erleben Sexualität in erster Linie als Schmerz. Es ist daher fraglich, ob diese Frauen den Geschlechtsverkehr künftig genussvoll erleben können. Da aber der gesamte Eingriff positiv konnotiert ist, ist zu beobachten, dass Frauen die Schmerzen ohne sich zu beschweren ertragen, da dies eine Umgangsform ist, die ihnen bereits als Mädchen am Tag der Beschneidung selbst vermittelt wurde (vgl. Lightfoot-Klein 2003).

Aussagen in Bezug auf die Orgasmusfähigkeit der Frauen sind kaum möglich, da ein Orgasmus von mehr als dem Vorhandensein einer unversehrten Klitoris. Dies zeigt sich schon daran, dass es unzählige Publikationen zur vermeintlich gestörten Orgasmusfähigkeit von unbeschnittenen Frauen gibt (vgl. Buddeberg 2003). Aus Interviews mit Infibulierten geht in der Dissertation Asefaws hervor, dass keine allgemein gültigen Aussagen zur sexuellen Empfindsamkeit der Frauen gemacht werden können (Asefaw 2008, S. 62). Manche der Befragten geben, an ein erfülltes Sexualleben zu haben, andere wiederum betrachten den Akt als notwendiges Übel, das der sexuellen Befriedigung des Ehemannes und der Zeugung von Nachwuchs dient (ebd.). Auch Lightfoot-Klein (2003) verweist in ihrem Buch darauf, dass infibulierte Frauen nicht gezwungenermaßen an sexueller Abneigung und/oder einer Unfähigkeit zum Orgasmus leiden. Sexuelles Lustempfinden und Erregbarkeit empfinden auch infibulierte Frauen, da dies von vielen weiteren Faktoren abhängig ist als nur von einer intakten Vulva und Scheide (Lightfoot-Klein 2003, S.85ff.). Dennoch ist nicht von der Hand zu weisen, dass es bei infibulierten Frauen zu Beeinträchtigungen während des vaginalen Geschlechtsverkehrs kommt. Wie stark Frauen sich davon negativ beeinträchtigt fühlen, kann von Außen nicht bewertet werden.

$\mathrm{Zu}$ den möglichen psychischen Konsequenzen weiblicher Genitalbeschneidung existieren kaum Studien, weshalb die nachfolgenden Ausführungen an vielen Stellen oberflächlich bleiben. 
Aus Lightfoot-Kleins (2003) Beobachtungen lassen sich psychische Merkmale zusammenfassen, die bei den Mädchen nach dem Eingriff auftreten und sich besonders darin äußeren, dass sich die Mädchen emotional zurückziehen, ruhiger und introvertierter werden. Auch in Hulverscheidts Dissertation finden sich Angaben zu den beobachtbaren psychischen Folgen. So würden Frauen unter Panikattacken (zum Beispiel beim Anblick von Gegenständen, die sie an den Tag ihrer Beschneidung erinnern), Depressionen und Angststörungen leiden (Bauer/Hulverscheidt 2003, S. 71).Ebenso könne sich der Eingriff in mangelndem Selbstbewusstsein und -vertrauen, psychosomatischen Folgen, sexuellen Störungen und Aggressionen niederschlagen (vgl. Lightfoot-Klein 2003; Bauer/Hulverscheidt 2003). 2004 führte die Psychologin Alice Behrendt im Rahmen ihrer Diplomarbeit eine Untersuchung zu den psychischen Folgen von Mädchenbeschneidung im Senegal durch. Mithilfe von Beobachtungen beschnittener Frauen sowie der Befragung Betroffener mittels Fragebogen zeigte sich, dass Mädchenbeschneidung als ,Posttraumatische Belastungsstörung ' einzuordnen ist, die akute psychische Folge wie „Unruhezustände, Schlaflosigkeit, Beklemmung, Alpträume und Panik“ (zitiert n. Baasher 1979 in: Behrendt 2004, S. 31) nach sich ziehen kann. Auch die Bundesärztekammer spricht von einem ,schwerwiegenden unauslöschbaren körperlichen und seelischen Trauma“ (Bundesärztkammer 2013), das sich unter anderem im Vertrauensverlust der Mädchen gegenüber Bezugspersonen äußern kann. Die Verletzungen der Seele werden jedoch von Betroffenen wenig benannt, weshalb fundierte Aussagen zu den psychischen Folgen weiblicher Beschneidung oft nicht möglich sind. Der Grund für den Mangel an Studien vermutet Lightfoot-Klein in der Haltung Betroffener gegenüber dem Eingriff. So würde von ihrem familiären wie sozialen Umfeld verlangt, dass die Mädchen den Schmerz ertragen, dann erst gelten sie ,als mutig, stark und wertvoll“" (Lightfoot-Klein 2003, S. 83). Dasselbe Argument wird herangezogen, wenn es um das Ertragen der langfristigen Folgen geht.

Abschließend stellt sich die Frage, weshalb weibliche Genitalbeschneidung praktiziert wird. Es existieren mögliche Begründungsmuster, die in der Literatur vermehrt wiederzufinden sind. Begründungen wie weibliche Genitalbeschneidung sei Teil der ,Kultur' oder würde von Männern gefordert, werden zur Rechtfertigung in der Literatur genannt (vgl. Schnüll 2003; Herrmann 2000). Es ist jedoch darauf hinzuweisen, dass es sich dabei um Literatur handelt, die aus ,westlich', häufig feministisch geprägter Perspektive heraus das Phänomen weiblicher Genitalbeschneidung beschreibt und bewertet. Gerade aus feministischer Perspektive wird weibliche Genitalbeschneidung teilweise als Beispiel für weibliche Unterdrückung instrumentalisiert, um die Unterdrückung der Frau durch patriarchale Systeme zu verdeutlichen. Diese Perspektive deckt sich jedoch nicht zwingend mit der aller betroffener Frauen, da diese sich in der Regel weder als ,Opfer ${ }^{6}$ noch als ,verstümmelt‘ betrachten (Dirie 2003, S. 104). In diesem Zusammenhang 
weist Dirie (2003) auch darauf hin, dass, ,weibliche Beschneidung [meist] als separater Tatbestand betrachtet" (Dirie 2003, S.105) wird, dabei jedoch nicht beachtet werde, dass beschnittene Frauen neben weiblicher Genitalbeschneidung noch mit weiteren Problemen zu kämpfen hätten.

\subsection{Gesetzgebung}

In der Mehrzahl der afrikanischer Länder, in denen weibliche Beschneidung praktizierende Bevölkerungsgruppen leben, existieren Gesetze, die die Praktik verbieten (Kalthegener 2003, S. 203ff.). In einigen Ländern fällt die Praktik unter Körperverletzung oder schwere Körperverletzung, weil kein eigenständiger Paragraph formuliert wurde. Die Beobachtungen der letzten Jahrzehnte zeigen aber, dass Mädchen allein durch die in ihrem Heimatland bestehenden Gesetz nicht zwingend vor Genitalbeschneidung geschützt sind (ebd., S. 214). Die Umsetzung der Gesetze gestaltet sich teilweise schwierig, was zum einen an mangelndem Wissen innerhalb der Bevölkerung bezüglich der gesetzlichen Regelung liegt, zum anderen auch daran, dass die gesetzlichen Regelungen ignoriert werden (Kalthegener 2003, S. 214). Des Weiteren bewirken Gesetze allein nicht, dass Menschen den Eingriff zu hinterfragen beginnen. Parallel zu den gesetzlichen Regelungen bedarf es Maßnahmen, die einen Prozess des Umdenkens bei den Menschen anregen, um Mädchen in Zukunft vor Beschneidung zu schützen (Dirie 2003, S. 107).

Seit 2001 hat das Europäische Parlament die einzelnen Mitgliederstaaten aufgefordert, gemeinsam an einem Gesetz zu arbeiten, das Mädchenbeschneidung verbietet (Kalthegener 2003, S. 214). Die Länder, die ein solches Gesetz zuerst verabschiedet haben, sind Belgien, Dänemark, England, Italien, Norwegen, Österreich, Schweden und Spanien, bei den restlichen Staaten fiel weibliche Genitalbeschneidung unter Körperverletzung - so wie lange Zeit auch in Deutschland (ebd.). Im Jahr 2012 wurde dann in der Schweiz ein entsprechendes Gesetz verabschiedet, 2013 eines in Deutschland. ${ }^{7}$ Doch auch in Europa gestaltet sich die Umsetzung der rechtlichen Regelungen schleppend. Els Leye untersuchte im Rahmen ihrer Dissertation die Situation der von Genitalbeschneidung betroffenen Migrantinnen in Europa (vgl. Leye 2008). Einer der Schwerpunkte dieser Untersuchung lag auf der Betrachtung des gesetzlichen Umgangs mit weiblicher Beschneidung. Hierzu wurden insgesamt 15 EU-Länder miteinander verglichen (Leye 2008, S. 57). Auf gesetzlicher Ebene ist laut Leye zwar zu beobachten, dass viele europäische Länder dazu übergegangen sind, die Praktik gesetzlich zu verbieten. Ver-

7 Zum Zeitpunkt der Datenerhebung (2011/12) bestand noch kein Gesetz in Deutschland, das weibliche Genitalbeschneidung explizit als Straftatbestand nennt. 
dachtsfälle werden jedoch nicht geprüft und nur selten strafrechtliche Konsequenzen erwirkt (ebd., S. 62). Vor diesem Hintergrund erachtet Leye es effektiver, medizinisches, juristisches und pädagogisches Fachpersonal im Umgang mit der Thematik zu schulen und ihnen im Rahmen dieser Schulungen aufzuzeigen, wie im Ernstfall zu reagieren ist. Aus ihrer Sicht könnte dies ein Ansatz sein, der bedrohte Mädchen langfristig schützt. Gesetze allein könnten das nicht (Leye 2008, S. 65).

Noch bis heute ist drohende weibliche Genitalbeschneidung kein anerkannter Fluchtgrund. Frauen, die aus ihren Heimatländern fliehen, um sich selbst oder ihre Töchter vor dem Eingriff zu schützen, wird in Deutschland bei Angabe dieses Grundes nur selten Asyl gewährt (Lünsmann 2003, S. 217). Begründet wird diese Entscheidungen unter anderem damit, dass in den jeweiligen Heimatländern genitale Beschneidung von Mädchen und Frauen verboten sei, Frauen demnach auch im Heimatland ausreichend geschützt seien - eine Einschätzung, die, wie Kalthegener in ihren Ausführungen zur Umsetzung bestehender Gesetze in afrikanischen Ländern aufzeigt, an der Realität vorbei geht. Laut Bundesamt für Statistik kann zwar Gemäß § 3 AsylVfG eine Flüchtlingsanerkennung erfolgen, wenn eine drohende Verfolgungsgefahr wegen der Zugehörigkeit zu einer bestimmten sozialen Gruppe festgestellt wird und die Verfolgungshandlung allein an das Geschlecht anknüpft (vgl. § 3b Abs. 1 Nr. 4 AsylVfG 1992) ist, doch hängt die Prüfung vom Einzelfall ab. Aus diesem Grund sind generelle Aussagen zu den Erfolgsaussichten eines entsprechenden Antrags aufgrund einer (drohenden) Genitalverstümmelung nicht möglich.

Da im Rahmen dieser Forschungsarbeit der Fokus auf der Bevölkerungsgruppe der Somalis liegt, wird nachfolgend in Kürze auf die Politik und Gesellschaft in Somalia eingegangen. Die Darstellung dient einem Überblick, der beim Verständnis der im Kontext der Arbeit durchgeführten Interviews hilft und die hohe Zahl somalischer Flüchtlinge weltweit erklärt. Des Weiteren wird auch die eingeschränkte gesellschaftliche Teilhabe von Frauen in Somalia aufzeigt, die letztlich zum Erhalt weiblicher Genitalbeschneidung beiträgt.

\subsection{Stand der Forschung}

Weibliche Genitalverstümmelung rückt seit etwa 35 Jahren mehr und mehr in den Fokus der internationalen Politik und der Menschenrechtspolitik. Davor war es eine Problematik, die außerhalb des afrikanischen Kontinents nur in anthropologischen und wenigen medizinischen Fachkreisen bekannt war. Das Engagement vieler Aktivist_innen und politischen Akteur_innen weltweit hat in den 1990er Jahren letztlich dazu geführt, dass die Praktik heute 
sowohl in der CEDAW (Bundesministerium für wirtschaftliche Zusammenarbeit 2014) als auch der Afrikanischen Charta der Menschenrechte (im Zusatzprotokoll zu Artikel 5, CEDAW 1990) sowie in der UNKinderrechtskonvention (Artikel 24, Absatz 3, UN-Kinderrechtskonvention 1989) als Praktik benannt ist, die gegen die Menschenrechte verstößt und vor der Mädchen und Frauen zu schützen sind. Neben der politischen Arbeit, die in den letzten Jahrzehnten geleistet wurde, existieren auch einige Arbeiten, die die Praktik aus medizinischer und aus geisteswissenschaftlicher Sicht beleuchten. Im Zentrum des wissenschaftlichen Interesses standen lange Zeit die physischen Auswirkungen des Eingriffs. Durch die Fokussierung auf die körperlichen Konsequenzen weiblicher Beschneidung kam es zu einer deutlichen Medikalisierung, was zur Folge hat, dass der Eingriff zunehmend in Kliniken durchgeführt wird und zu einer zusätzlichen Einnahmequelle für Mediziner innen wird (Terre des Femmes 2005, S. 9). Des Weiteren brachte die Fokussierung auf die medizinischen Konsequenzen mit sich, dass die wirtschaftlichen, sozialen und psychischen Folgen ignoriert wurden. Positiver Effekt dieser Stoßrichtung ist, dass heute eine vielseitige medizinische Behandlung beschnittener Frauen möglich ist, die auch komplizierte plastische Operationen, wie beispielsweise die Rekonstruktion der Klitoris und den inneren Schamlippen einschließen (vgl. Foldès 2006). Auch die Aufnahme genitaler Beschneidung in den Diagnoseschlüssel des $\mathrm{ICD}^{8}$ ist eine wichtige Errungenschaft vieler medizinischer Studien und engagierter Mediziner_innen (Bundesärztekammer 2013).

Meines Erachtens ist eine Annäherung aus unterschiedlichen Perspektiven bei einem derart kontrovers diskutierten Phänomen wie dem weiblicher Genitalbeschneidung, im Besonderen der Infibulation, nötig, um zu einem sensiblen und wertschätzenden Umgang mit bedrohten Mädchen und beschnittenen Frauen gelangen zu können.

Die Frauenrechtsorganisation Terre des Femmes hat im Jahr 2003 einen fundierten Sammelband zum Thema weibliche Genitalbeschneidung herausgegeben, der aus vielen einzelnen Beiträgen besteht, die Menschen unterschiedlicher Fachrichtungen und nationaler Herkunft geschrieben haben. In diesem nähern sich Anthropolog innen, Historiker innen sowie selbst betroffene Frauen dem Thema weiblicher Genitalbeschneidung und beleuchten das Phänomen aus ihrer jeweiligen Perspektive. Dieses Buch bietet einen sehr facettenreichen Einblick und verdeutlicht die unterschiedlichen Perspektiven, aus denen der Eingriff betrachtet und bewertet werden kann.

Die Dissertation der Medizinhistorikerin Marione Hulverscheidt bietet ebenfalls eine Perspektive auf weibliche Genitalbeschneidung, die sich von dem teilweise einseitigen Blick auf die praktizierenden Bevölkerungsgruppen

8 International Classification of Diseases and Related Health Problems ist ein weltweit anerkanntes Diagnoseklassifikationssystem, das von der Weltgesundheitsorganisation heraus gegeben wird. 
Afrikas abhebt und den Fokus auf weibliche Genitalbeschneidung im europäischen Raum legt (vgl. Hulverscheidt 2002). Hulverscheidts Dissertation zeigt, dass genitale Beschneidung von Mädchen auch in Europa bis ins 20. Jahrhundert praktiziert wurde. Als Begründung für diese Eingriffe (meist die Amputation der Klitoris) wurden vermeintliche Diagnosen wie Nymphomanie, Hysterie oder Masturbation herangezogen. Ziel der genitalen Beschneidungen war die Kontrolle weiblicher Sexualität, die mit dem Machtzuwachs der Mediziner_innen über Patientinnen ermöglicht werden konnte. Die Arbeit Hulverscheidts zeigt, dass weibliche Genitalbeschneidung kein Phänomen darstellt, das nur auf bestimmte Bevölkerungsgruppen Afrikas zu beschränken ist, sondern eines, das auch lange Zeit in Europa praktiziert wurde.

Außerdem ist die Dissertation der Medizinerin Fana Asefwas (2007) zu nennen. Asefaw hat ebenfalls Interviews mit betroffenen Frauen durchgeführt. Mithilfe dieser Interviews untersuchte sie, welche physischen und psychischen Konsequenzen mit weiblicher Genitalbeschneidung einhergehen und welche Erfahrungen betroffene Frauen im deutschen Gesundheitswesen gemacht haben (vgl. Asefaw 2007). Um Aussagen dazu treffen zu können, führte sie insgesamt 470 Interviews in Eritrea und 98 unter in Deutschland lebenden, betroffenen eritreischen Migrantinnen durch. Ihre Ergebnisse zeigen, dass die überwiegende Mehrheit betroffener Mädchen und Frauen negative Erfahrungen mit deutschen Gynäkolog_innen gemacht haben (Asefaw 2008, S. 74ff.). In ihren Schlussfolgerungen betont Asefaw daher, wie wichtig es sei, Mediziner_innen im Umgang mit der Thematik und den kulturellen Hintergründen der Praktik zu schulen (ebd., S. 79). Des Weiteren kommt sie zu dem Ergebnis, dass die interviewten eritreischen Frauen in Deutschland mehr an psychischen Folgen leiden als diejenigen, die in Eritrea geblieben sind, was Asefaw mit dem im Einwanderungsland aufkommenden Sinnverlust bezüglich des Eingriffs erklärt (Asefaw 2008, S. 69). Auch wenn Asefaws Erklärung nachvollziehbar ist, bleibt dennoch fraglich, ob beschnittene Mädchen und Frauen nicht schon im jeweiligen Heimatland psychische Konsequenzen von dem Eingriff davongetragen haben, diese aber nicht thematisiert werden (können). Dies zeigt jedoch, dass künftig die psychischen Konsequenzen des Eingriffs eingehender erforscht werden müssen.

Neben der Dissertation Asefaws existiert eine weitere Untersuchung, in der betroffene und deren Familien befragt wurden. Die von Plan International herausgegebene Studie „Listen to African Voices“ wurde mithilfe von Multiplikator_innen und der Psychologin Alice Behrendt durchgeführt. Im Rahmen dieser Studie wurden mehr als 1700 ursprünglich aus westafrikanischen Staaten nach Hamburg immigrierte Menschen zum Thema weibliche Genitalbeschneidung befragt. Die Untersuchung hat ergeben, dass die überwiegende Mehrheit der Befragten zu den negativen Konsequenzen des Eingriffs informiert ist und sich deutlich gegen weibliche Genitalbeschneidung ausspricht (Behrendt 2010, S. 97 ff.). Nur wenige haben angegeben für die Wei- 
terführung der Praktik zu sein. Am stärksten davon gefährdet, beschnitten zu werden, sind diejenigen Mädchen, die ohne ihre Eltern bei Verwandten im jeweiligen Heimatland aufwachsen, da der Eingriff dann von den Familienmitgliedern entschieden wird, bei denen die Mädchen wohnen. Diejenigen Mädchen, die in Hamburg geboren und aufgewachsen sind, laufen dagegen weniger Gefahr beschnitten zu werden. Laut Aussagen der Befragten würden in Hamburg keine Mädchen beschnitten, da die Bedingungen dies nicht zuließen (wie beispielsweise das gesetzliche Verbot weiblicher Genitalbeschneidung). Planten Familien aber ihre Töchter genital beschneiden zu lassen, würde der Eingriff im jeweiligen Heimatland stattfinden (ebd., S. 100).

Neben den genannten Interviews mit beschnittenen Frauen existieren auch Befragungen, die sich an medizinische Fachkräfte in Deutschland richteten, mit dem Ziel zu untersuchen, inwieweit diese Kontakt zu beschnittenen Frauen haben und ob sie ausreichend über weibliche Genitalbeschneidung informiert sind. So wurde im Jahr 2005 von Terre des Femmes, UNICEF und dem Berufsverband der Frauenärzte (BVF) eine Studie durchgeführt, die sich an Gynäkolog_innen in Deutschlands richtete. Ingesamt erhielten 13182 den quantitativen Fragebogen, der von 493 Gynäkolog_innen beantwortet wurde. Die Auswertung ergab, dass insgesamt 43 Prozent der Gnäkolog_innen bereits eine beschnittene Frau behandelt hatten und rund 30 Prozent der Ärzt_innen eine beschnittene Frau während einer Geburt begleiteten. Insgesamt sprachen sich 431 der Befragten (87,4 Prozent) dafür aus, das Thema weibliche Genitalbeschneidung im Rahmen von Fortbildungen und Seminaren zu behandeln (vgl. Berufsverband der Frauenärzte/Terre des Femmes/UNICEF (Hg.) 2006, S. 6).

Auch Jana Graf (2012) hat im Rahmen ihrer medizinethischen Dissertation untersucht, ob beziehungsweise in welcher Häufigkeit niedergelassene Gynäkolog_innen in Berlin mit beschnittenen Frauen zu tun haben und ob sie ausreichend zum Umgang mit ihnen informiert sind. Mittels standardisierter Fragebogenerhebung ging Graf dieser Frage nach und kam zu dem Ergebnis, dass Gynäkolog_innen in Berlin in beträchtlichem Ausmaß mit beschnittenen Frauen zu tun haben. Gleichzeitig habe die Befragung jedoch gezeigt, dass die befragten Gynäkolog_innen nur über unzureichendes Wissen in Bezug auf weibliche Genitalbeschneidung verfügten. Dies zeige sich laut Graf besonders daran, dass nahezu 40 Prozent der Befragten angeben haben, Frauen sollten selbst darüber entscheiden, ob sie beschnitten werden wollten oder nicht (Graf 2012, S. 147ff.) Aus dieser Aussage schließt Graf, dass ein Teil der befragten Gynäkolog_innen nicht über die Tatsache informiert sei, dass Genitalbeschneidung meist im Kindesalter praktiziert würde. Des Weiteren gibt der Großteil der befragten Gynäklog_innen an, während des Studiums keine Informationen zu weiblicher Genitalbeschneidung bekommen zu haben, weshalb sich 81 Prozent der Befragten wünscht, dass weibliche Genitalbeschneidung Teil des Studiums wird (ebd.). Interessant ist, dass Gynäko- 
log_innen angaben, dass ihnen Fälle bekannt seien, in denen Mädchen in Deutschland beschnitten wurden. Dies bestätigt die Vermutung, dass Genitalbeschneidung auch in Deutschland praktiziert wird. Des Weiteren zeigen die Ergebnisse Grafs, dass die überwiegende Mehrheit der befragten Gynäkolog_innen keine Organisation oder Einrichtung kennt, die gegen genitale Beschneidung tätig ist (ebd.).

Weibliche Genitalbeschneidung wurde außerdem aus sozialwissenschaftlicher Perspektive untersucht. So erforschte Diana Kuring weibliche Genitalbeschneidung aus politikwissenschaftlicher Perspektive (vgl. Kuring 2007). Kuring beschreibt darin die Situation betroffener Eritreerinnen in ihrem Heimatland und beleuchtet die unterschiedlichen Vorgehensweisen im Kampf gegen diese Praktik in Eritrea (Kuring 2007, S. 16). Aus Sicht der Bevölkerung wird weibliche Genitalbeschneidung laut dieser Untersuchung vorwiegend als identitätsweisender und notwendiger Bestandteil der weiblichen Entwicklung angesehen. Vertrer_innen staatlicher Organe und NichtRegierungsorganisationen sehen weibliche Beschneidung jedoch vor allem als nicht indizierten medizinischen Eingriff, der nicht nur der weiblichen Bevölkerung schadet, sondern auch der Entwicklung des gesamten Landes unzuträglich ist (ebd., S. 241). Somit findet laut Kuring sowohl auf nationaler als auch internationaler Ebene vermehrt Aufklärungsarbeit statt. Nationale Organisationen weisen dabei vor allem auf die medizinischen Konsequenzen hin, während bei der Arbeit der Vereinten Nationen die menschenrechtliche Perspektive im Vordergrund steht (ebd., S. 241).

Die Dissertation der belgischen Kulturwissenschaftlerin Els Leye untersucht die Situation der von weiblicher Beschneidung betroffenen Migrantinnen in Europa (vgl. Leye 2008). Der Schwerpunkt dieser Untersuchung lag auf der Betrachtung des gesetzlichen Umgangs mit weiblicher Genitalbeschneidung und des Umgangs mit der Thematik im Gesundheitswesen. Hierzu wurden insgesamt 15 EU-Länder miteinander verglichen (Leye 2008, S. 57). Die Ergebnisse Leyes zeigen zwar, dass weibliche Genitalbeschneidung in Europa in den letzten Jahrzehnten mehr an öffentlicher Aufmerksamkeit erfahren hat. Dennoch besteht insbesondere auf Seiten des medizinischen Fachpersonals noch unzureichende Kenntnis im Umgang mit Betroffenen (ebd., S. 63).

Die Diplomarbeit von Antje-Christine Büchner beleuchtet die Thematik aus sozialpädagogischer Sicht (vgl. Büchner 2004). Besonders interessant an dieser Arbeit sind die Ausführungen zur Situation betroffener und bedrohter Mädchen und Frauen in Deutschland. Hier beschreibt Büchner, dass sich betroffene Mädchen und Frauen häufig in einem Konflikt befänden, da der Eingriff als Teil ihrer Identität empfunden werde, mit dem sie im jeweiligen Heimatland aufgewachsen seien und der ihnen als positive Praktik vermittelt wurde. Auf der anderen Seite erlebten sie die in Deutschland stattfindende öffentliche Debatte, in der weibliche Genitalbeschneidung als grobe Men- 
schenrechtsverletzung und folgenschwere Frauendiskriminierung diskutiert werde (Büchner 2004, S. 109). Laut Büchner würden diese Mädchen und Frauen jedoch nicht nur mit diesem Konflikt alleine gelassen, sondern ebenso mit den Konsequenzen der Beschneidung ihrer Genitalien. Die Beratungssituation für diese Mädchen und Frauen stecke noch in den Anfängen und könne Betroffene oder Bedrohte bislang nur punktuell erreichen. Die Mädchen und Frauen selbst wüssten meist nicht, wohin sie sich wenden und wo sie kompetente Beratung erhalten könnten (ebd., S. 112).

Auch meine im Jahr 2006 verfasste Diplomarbeit zum Umgang mit weiblicher Genitalbeschneidung in Deutschland und Frankreich befasste sich aus sozialpädagogischer Perspektive mit dem Phänomen. In diesem Rahmen wurden deutsche und französische Beratungsstellen mittels quantitativem Fragebogen befragt, mit dem Ziel zu erfassen, wie sich die Aufklärungs- und Beratungsarbeit gegen weibliche Genitalbeschneidung in den beiden Ländern gestaltet. Die Ergebnisse zeigten, ähnlich denen Büchners, dass die in Deutschland bestehenden Einrichtungen die Zielgruppe der bedrohten und betroffenen Mädchen mit ihren Aufklärungs- und Beratungsangeboten häufig nicht erreichen. Zwar existieren etliche Angebote für bedrohte und betroffene Mädchen und Frauen, doch werden diese kaum genutzt. Die Ergebnisse meiner Diplomarbeit waren letztendlich Ausgangspunkt für die im Rahmen dieser Forschungsarbeit durchgeführte Befragung weiterer Einrichtungen und Kliniken unterschiedlicher europäischer Länder, um herauszufinden, welche Herangehensweisen zu einem nachhaltigeren Kontakt zu bedrohten und betroffenen Mädchen und Frauen führen können.

Die soziologische Dissertation von Janne Mende (2011) diskutiert weibliche Genitalbeschneidung vor dem Hintergrund des Kulturrelativismus und Universalismus. Aus der Perspektive von Kulturrelativisten wird weibliche Beschneidung als ,kulturelle Tradition“ betrachtet und zu einem Aspekt der pluralen ,Kultur'. Das Recht auf eigene ,Kultur', eigene Sprache, eigene Religion und eigene Identität widerspricht der Universalität der Menschenrechte - weder der Universalismus (der Menschenrechte) noch der Kulturrelativismus können hier eine Lösung oder Vermittlung herbeiführen. Mende beschreibt in ihrer Dissertation das Verhältnis und die Möglichkeiten dieser beiden Konzepte, um dann ein vermittlungslogisches Modell einer reflexiven Sozialwissenschaft vorzuschlagen. Darin eröffnet sie eine freiheitstheoretische Perspektive in Anlehnung an die Kritische Theorie, in der weder die eine noch die andere Seite überhöht und in der weder unsensibel noch relativistisch argumentiert wird. Sie schlägt einen reflexiven Zugang vor, der alle Positionen im Blick behält gleichzeitig den eigenen Anspruch nicht auflöst. Vielmehr soll durch das Beziehen einer eigenen klaren Position in einer Diskussion auch dem Gegenüber ermöglicht werden, unter Abwägung der verschiedenen Einflussgrößen eine neue eigene Position zu beziehen. Ziel ist, 
die repressiven Dimensionen der Diskussion zu überwinden und die emanzipatorischen Positionen zu stärken.

Die vorliegende Dissertation fokussiert das Phänomen weiblicher Genitalbeschneidung aus sozialwissenschaftlicher Perspektive. Um einen möglichst facettenreichen Einblick in die Thematik zu bekommen, werden mithilfe qualitativer und quantitativer Erhebungsmethoden unterschiedliche Sichtweisen auf das Phänomen erfasst und dargestellt. Besonderes Augenmerk wird auf der Perspektive beschnittener somalischer Frauen liegen, die in einem Einwanderungsland leben, das weibliche Genitalbeschneidung ablehnt. Dabei handelt es sich um eine Perspektive, die, wie aus dem Forschungsstand hervorgeht, bislang nur wenig Gegenstand des wissenschaftlichen Interesses war. Das Erkenntnisinteresse liegt darauf zu untersuchen, welche Strategien die befragten Frauen in einer weibliche Genitalbeschneidung ablehnenden Gesellschaft im Umgang mit dem Phänomen entwickelt haben. Da es sich bei dem Eingriff um ein gesellschaftlich etabliertes Phänomen handelt, wird neben den befragten somalischen Frauen auch die Meinung somalischer immigrierter Männer zum Ausdruck kommen. Welche Sichtweise sie auf weibliche Genitalbeschneidung haben und ob sich diese nach der Migration in ein Land, das den Eingriff ablehnt, verändert hat, ist dabei von besonderem Interesse. Im Rahmen einer quantitativen Erhebung wird schließlich die Perspektive derjenigen Fachkräfte analysiert, die in einer Einrichtung oder Klinik gegen weibliche Genitalbeschneidung arbeiten. Diesbezüglich wird das primäre Erkenntnisinteresse darauf liegen, den Umgang verschiedener Beratungseinrichtungen mit beschnittenen Frauen und bedrohten Mädchen zu erforschen und zu untersuchen, welche Konzepte sich diesbezüglich als besonders geeignet ergeben haben. Die Untersuchungen werden in den Einwanderungsländern Deutschland, England, Italien und der Schweiz durchgeführt. Da die Perspektive der befragten beschnittenen Frauen im Zentrum dieser Arbeit steht, wird die theoretische Rahmung der Arbeit eng an die Analyse der qualitativen Interviews geknüpft sein. Anhand der Aussagen der befragten Frauen werden sozialwissenschaftliche Theorien zur theoretischen Einbettung derselben herangezogen. 


\section{Zum politischen und gesellschaftlichen Kontext in Somalia und den Einwanderungsländern}

Die Wahl im Kontext der vorliegenden Dissertation Menschen somalischer Herkunft in den Mittelpunkt zu stellen, bringt notwendigerweise mit sich sowohl deren Herkunfts- als auch Einwanderungsland näher zu betrachten. Nachfolgend wird daher der Blick auf die gesellschaftliche und politische Situation Somalias gerichtet, die letztendlich auch verdeutlicht, weshalb sich so viele somalische Menschen in Migration befinden. Des Weiteren ist die Betrachtung des Herkunftslands der im Rahmen dieser Arbeit interviewten Personen von Bedeutung, um das Phänomen weiblicher Genitalbeschneidung und die dahinterstehenden Konstruktionen und Zuschreibungen verstehbar zu machen. Der Blick auf die Migrationspolitik der jeweiligen Einwanderungsländer zeigt wie diese mithilfe von repressiven Maßnahmen und der Kriminalisierung von Flüchtlingen versuchen Migration zu kontrollieren und einzuschränken. Eine Politik, die sich auch auf die im Land lebenden, legalen“ Migrant_innen auswirkt.

\subsection{Politische und gesellschaftliche Situation in Somalia}

Somalia liegt ganz im Osten Afrikas und wird von circa acht Millionen Menschen bewohnt. Es blickt auf eine Kolonialzeit zurück, die Spuren hinterlassen hat, die beispielsweise in der Aufteilung des Landes noch bis heute zu erkennen sind (Schicho 2004, S. 254). Nach dem Ende der Kolonialzeit 1960 erhielt Somalia die ehemaligen fremd besetzten Gebiete nicht vollständig zurück (Birnbaum 2007, S.289). Nach einem Militärputsch 1969 kam Muhammad Siad Barré an die Macht, der das Land in diktatorischem Stil bis 1991 führte. In diesem Jahr kam es dann zum Sturz des Siad Barrés. Seitdem ist Somalia überschattet von einem Bürgerkrieg zwischen sich bekriegenden, tief verfeindeten Klans und Kriegsherren, die um die politische und religiöse Vorherrschaft streiten (Birnbaum 2007, S. 87 ff). Der Zerfall des somalischen Volkes in verschiedene Klans und Warlords hat zur Folge, dass Somalia lange Zeit keine funktionierende Regierung besaß und die Schaffung einer solchen bis heute andauert. Dies ist darauf zurückzuführen, dass sich neben den Klans und Warlords immer mehr religiöse Fanatiker und islamische Fundamentalisten am Machtstreit beteiligen und für kriegerische Auseinandersetzung sorgen. 2011 gelang es der Übergangsregierung und den Afrikanischen Friedenstruppen die fundamentalistischen Gruppierungen aus der Hauptstadt zu verdrängen, im selben Jahr wurde Hassan Sheikh Mohamud 
als Ministerpräsident vereidigt (Frankfurter Allgemeine Zeitung 2013). Seither wird versucht das Land $\mathrm{zu}$ befrieden, was jedoch immer wieder von Rückschlägen geprägt ist (Bundeszentrale für politische Aufklärung 2014). Der lang andauernde Bürgerkrieg und die daraus resultierende politische Instabilität sowie die wirtschaftliche und soziale Misere im Land haben zur Folge, dass sich Somalia unter den zehn Hauptflüchtlingsländern der Welt befinden. Unter den Hauptaufnahmeländern liegen neben Kenia und Äthiopien, auch europäische Länder, die USA und Kanada.

Die Gesellschaft Somalias ist stark von einem Klanwesen geprägt, das bis ins Hochmittelalter (11. bis 13. Jahrhundert) zurückgeht und zeitgleich mit dem Islam in Somalia Einzug hielt (Birnbaum 2002). Das Klansystem „,ist eine der ältesten Institutionen der somalischen Hirtengesellschaft, ein System das Frauen auf direkte und indirekte Weise diskriminiert" (Dini 2008, S. 102). Insgesamt existieren sechs Klanfamilien. Mehrere Klans bilden eine Klanfamilie, während jeder Klan wiederum in verschiedene Erbschaftslinien, Subklans oder Subsubklans aufgeteilt ist (Mutschler 2001, S. 32). Ein Klan geht auf einen Gründungsvater zurück, nachdem er benannt wird. Die größten und einflussreichsten Klans sind die „Darud“, „Hawiye“, „Isak“, „Dir Digil" und „Rahanwajin“ (Birnbaum 2002, S. 33). Die Mehrheit der Klanfamilien lebt als Nomaden, nur wenige haben sich als sesshafte Bauern in einer Region niedergelassen. Die Feindschaft unter den Klanfamilien begründet sich im Kampf um fruchtbares Land (ebd.). Das Klansystem bietet seinen Mitgliedern einen geschützten Rahmen, doch diejenigen, die nicht Teil des Systems sind oder einem weniger einflussreichen Klan angehören, werden benachteiligt und ausgeschlossen (Dini 2008). Hat eine Familie mit irgendwelchen Schwierigkeiten zu kämpfen, kann sie sich der Hilfe ihrer Klanbrüder sicher sein, der Klan „dient als eine Art Versicherung“ (Dini 2008, S. 102). Im gesamten Klanwesen spielen ausschließlich männliche Vorfahren und Mitglieder eine Rolle: sie geben den Namen und die Klanzugehörigkeit an die Kinder weiter, das Oberhaupt eines Klans ist männlich und nur Männern ist es vorbehalten ein Leben lang Mitglied des Klans zu sein und dessen umfassenden Privilegien zu genießen (ebd.). Mädchen und Frauen kommen in dieser Hierarchie an zweiter Stelle, da sie zwar zeitweilig ebenfalls Mitglied des Klans sind, jedoch damit gerechnet werden kann, dass sie einen Mann eines anderen Klans heiraten werden. Wen die Frauen heiraten entscheiden die Klanführer (Dini 2008). Die somalische Politikwissenschaftlerin Shukria Dini führte in den Jahren 1999, 2005 und 2006 Feldforschungen in verschiedenen kenianischen Flüchtlingslagern durch und führte Interviews mit somalischen Frauen, mit dem Ziel die Position der Frauen in der somalischen Gesellschaft, die von Krieg und Hunger gekennzeichnet ist, zu erkun- 
den. ${ }^{9}$ Ihre Forschungen ergaben, dass somalische Familien selbst in Kriegszeiten an patriarchal geprägten Strukturen festhalten (ebd.). In diesen genießen die Männer hohes Ansehen, schon kleine Jungen werden den Mädchen der Familien vorgezogen. Mädchen und Frauen haben nur wenige Rechte, ihnen wird wenig Aufmerksamkeit zuteil und sie haben kaum Zugang zu Privilegien. So gehört auch Schulbildung zu einem Privileg, das Mädchen meist vorenthalten wird (ebd.). Es existieren zudem Gepflogenheiten, die die Rechte der Frauen massiv verletzen. So werden beispielsweise Frauen an verfeindete Klans verschenkt, um weitere kriegerische Auseinandersetzungen zu vermeiden (ebd.). Dieser Brauch geht laut Dini auf das sehr alte Nomadenrecht zurück, das unter Hirtengesellschaften wie der somalischen vorherrscht. Hintergrund dieses Brauches ist die Wiedergutmachung begangener Taten, bei denen vom verfeindeten Klan Männer ums Leben gekommen sind. Die verschenkte Frau kann mit ihrer Gebärfähigkeit dafür sorgen, dem Klan wieder neue männliche Mitglieder zu gebären (ebd.). Die Frauen, die als Wiedergutmachung verschenkt werden, verfügen im verfeindeten Klan über wenig Ansehen, nicht selten sind sie von „Gewalt und Misshandlung" (Dini 2008, S. 104) betroffen, insbesondere, wenn sie nicht, wie von ihnen erwartet, den erwünschten männlichen Nachwuchs gebären (Dini 2008).

Auch weibliche Genitalbeschneidung ist Gewalt an Mädchen und Frauen. Das Phänomen weiblicher Genitalbeschneidung wird häufig mit dem Islam in Verbindung gebracht, obwohl die Praktik nicht Teil des Islams (oder anderen Religionen) ist. Dini verweist in ihrem Artikel darauf, dass sich in Somalia Islam und Nomadenrecht miteinander vermischen. So spricht der Koran Männern und Frauen Rechte und Pflichten zu, die wiederum vom Nomadenrecht missachtet werden. So wird beispielsweise im Islam durchaus von Frauen erwartet, sich Wissen anzueignen, und ihnen zugesprochen, Bildung zu erhalten oder Eigentum zu besitzen (Dini 2008). Warum Mädchen und Frauen in Somalia Gewalt und Unrecht zuteil wird, ist aus Dinis Sicht eine Mischung aus den Fehlinterpretationen des Korans (insbesondere des islamischen Rechts, der Scharia), den patriarchalen Strukturen des Klanwesen und Nomadenrechts.

Menschen somalischer Herkunft gehören laut UNO mit circa 1,1 Millionen zu der drittgrößten Gruppe von Flüchtlingen weltweit (UNO 2013). Die eben beschriebene politische und gesellschaftliche Situation in Somalia verdeutlicht, weshalb so viele Menschen aus Somalia fliehen und ein Leben jenseits von Gewalt, Armut und Hunger suchen. Die meisten Somalis fliehen innerhalb ihres Landes oder in die direkt angrenzenden Gebiete Kenias oder Äthiopiens. Nur diejenigen, die es sich leisten können, treten den gefährlichen Weg nach Europa an.

9 Es existieren zahlreiche Veröffentlichungen zur politischen Situation und der Piraterie am Horn von Afrika, die spezifische Situation der Frauen des Landes wird darin jedoch nicht beschrieben. Daher stützt sich dieser Teil auf Dinis Forschungen. 


\subsection{Zum Migrationsbegriff}

Nachfolgend wird die Entwicklung der Migrationspolitik der im Rahmen dieser Arbeit fokussierten Einwanderungsländer Deutschland, der Schweiz, England und Italien dargestellt. Diese ist im Kontext weiblicher Genitalbeschneidung von Bedeutung, da es sich bei betroffenen und bedrohten Mädchen und Frauen um Migrantinnen oder Flüchtlinge handelt, die unter Umständen direkt von der Migrationspolitik des jeweiligen Einwanderungslands betroffen sind. Bevor jedoch die Politik der einzelnen Einwanderungsländer dargestellt wird, wird in Kürze auf den Begriff ,Migration“ eingegangen, um $\mathrm{zu}$ verdeutlichen, dass es sich keineswegs um einen neutralen Ausdruck zur Beschreibung weltweiter Wanderbewegungen handelt (Tsianios 2010, S. 1).

Definiert wird Migration als ,die auf einen längerfristigen Aufenthalt angelegte räumliche Verlagerung des Lebensmittelpunktes von Individuen, Familien, Gruppen oder auch ganzen Bevölkerungsgruppen“ (Oltmer 2013, S. 31). Die geographische Veränderung von Menschen findet aus unterschiedlichen Beweggründen statt, zielt jedoch meist darauf $a b$, „sich neue Chancen zu erschließen“ (ebd., S. 32). So genannte Zwangsmigrationen bilden darunter die Ausnahmen, da sie „durch die Nötigung zu Abwanderung verursacht" (ebd., S. 33) werden, wie beispielsweise durch Krieg oder durch „politische, ethnonationale, rassistische oder religiöse“ (ebd.) Verfolgung. Unabhängig davon, in welchem Kontext der Begriff Migration gebraucht wird (Arbeits-, Zwangs- oder Kriegsmigration), spiegelt er „herrschende Vorstellungen von Kontrolle" (Tsianios 2010) wider und ist eng mit territorialen Grenzziehungen und mit der grundsätzlichen Frage nach Zugehörigkeit verbunden. „Migration problematisiert Grenzen" (Mecheril 2004, S. 42), sowohl die nationalstaatlichen Grenzen wie auch die „symbolischen“ (ebd.) Grenzen, die sich innerhalb einer Gesellschaft wiederfinden und Menschen als Zugehörig beziehungsweise Nicht-Zugehörig klassifizieren.

Der Begriff Migrant_in wurde ursprünglich von Migrantenorganisationen geprägt, die mit dieser Benennung Abstand von dem Begriff ,Ausländer_in“ nehmen wollten Mecheril 2004, S. 48). Je nachdem in welchem Kontext der Begriff benutzt wird, fokussiert er unterschiedliche Aspekte, weshalb er nicht eindeutig zu definieren ist (ebd.). Im Kontext interkultureller Pädagogik verweist Mecheril (2004) darauf, dass die Bezeichnung Migrant in „von Diskursen um Identität, Fremdheit, ethnischen und kulturellen Differenzen hervorgebracht wird“" (Mecheril 2004, S.48). Der Blick auf die weltweit stattfindenden Wanderbewegungen der Menschen zeigt, dass die Binnenmigration, also die Wanderungen innerhalb der staatlichen Grenzen, am häufigsten vorkommt. In diesem Kontext wird jedoch nicht von Migration gesprochen, was darauf hinweist, dass die Bezeichnung Migrant_in nicht primär die damit verbundene Wanderbewegung betont, ,sondern eher den rechtlichen Status 
und eine vermutete und zugeschriebene Abweichung von Normalitätsvorstellung“ (ebd.). Das bedeutet, dass hinter der Bezeichnung Migrant_in „die Ordnung zwischen denen, die selbstverständlich dazu gehören, und denen, die nicht selbstverständlich dazu gehören“ (ebd.) steht. In den Diskursen um Zugehörigkeit spielen neben formellen Festlegungen (beispielsweise die Staatsangehörigkeit) "Aussehen und der Habitus eine dominante Rolle“ (ebd.). Neben dem Phänotyp entscheiden auch „para-phänotypische Signale“ (ebd., S. 52), menschliche Verhaltensmerkmerkmalen, auch "Seinsweise“ genannt (zitiert n. Bourdieu 1976, S. 446 in: Mecheril 2004, S. 52) über Zugehörigkeit oder Nicht-Zugehörigkeit. Diese Entscheidungskriterien beruhen auf „Imaginationen, Mythen und auch Rassismen“ (Mecheril 2004, S.52) und wirken sich auf zugewanderte Menschen, die aufgrund genannter Kriterien als ,Migrant_in' markiert werden, negativ aus.

\subsection{Zur Migrationspolitik Deutschlands}

Zu- oder Abwanderung ist kein Phänomen, das erst zu einem bestimmten Zeitraum in der Geschichte begonnen hat, vielmehr eines, das über die letzten Jahrhunderte immer wieder zu beobachten war und immer noch ist (Oltmer 2005). Jahrhunderte lang gingen Deutsche aus unterschiedlichsten Gründen ins Ausland oder verließen Menschen ihre Heimat, um nach Deutschland einzuwandern. Höhepunkt der Migrationsbewegung war die Zeit des Nationalsozialismus, als zwischen 1933 und 1945 Hundertausende Menschen aufgrund ihrer Religion, nationalen oder ,ethnischen ${ }^{10}$ Herkunft oder politischen Ansicht vertrieben wurden, fliehen mussten oder in Arbeitslager gezwungen wurden (ebd.). Nach Kriegsende kehrten über 20 Millionen Flüchtlinge und Vertriebene zurück in ein sehr viel kleineres Deutschland, was die Rückkehr und Integration erschwerte (ebd.). Nach dem Zweiten Weltkrieg wurden von 1955 bis 1973, so genannte ,Gastarbeiter' angeworben (MeierBraun 2013, S. 17), Arbeitskräfte aus dem Ausland, die in Deutschland in diesen Jahren dringend benötigt wurden. Trotz der Einwanderung von Millionen angeworbenen ,Gastarbeitern', verstand sich Deutschland lange Zeit nicht als Einwanderungsland, da davon ausgegangen wurde, dass die Arbeiter_innen wieder zurück in ihre Heimatländer gehen würden (ebd.). Somit wurde die Integration der eingewanderten Menschen lange Zeit aufgeschoben, obwohl den Politiker_innen bereits in den 1960er Jahren bewusst war,

10 Die Verwendung des Begriffs ,Ethnie‘ oder ,ethnisch“ wird im Rahmen dieser Arbeit vermieden, da es sich, wie auch bei den Begriffen ,Rasse' oder ,Kultur', um einen Begriff handelt, der Menschen in scheinbar naturgegebene biologische Gemeinschaften unterteilt. Ein Konstrukt, das von Macht- und Herrschaftsverhältnissen geleitet wird und zur Herabstufung bestimmter Bevölkerungsgruppen dient (Vgl. Kalpaka 2005). 
dass die Integration ein wichtiges Thema sein würde (ebd.). Die Wiedervereinigung, das Ende des Kalten Krieges und der Krieg im ehemaligen Jugoslawien ließ die Anzahl der Einwander_innen ansteigen, doch erst 1998 kam die politische Wende, als die damalige Regierung Deutschland als Einwanderungsland definierte (Heckmann 2013, S. 227). Diese Wende brachte mit sich, dass die ausländische Bevölkerung nicht länger ignoriert werden konnte, sondern die Politiker_innen sich mit der Zuwanderung und Integration der Menschen beschäftigen mussten (ebd.). Im Jahr 2005 wurde dann mit dem so genannten ,Zuwanderungsgesetz ' ein „Gesetz zur Steuerung und Begrenzung der Zuwanderung und zur Regelung des Aufenthaltes und der Integration von Unionsbürgern und Ausländern (Zuwanderungsgesetz)“ (Meier-Braun 2013, S. 19) beschlossen. Dessen zentrale Bestandteile waren außerdem „die Förderung der Integration als eine Aufgabe des Bundes" (Butterwegge 2005), wie beispielsweise „Integrationskurse zur Vermittlung deutscher Sprach- und Gesellschaftskenntnisse“ (ebd.), mit dem Ziel Einwander_innen ein langfristiges Leben in Deutschland zu ermöglichen. Die neue Regelung brachte neue Aufenthaltstitel hervor, wie „die befristete ,Aufenthaltserlaubnis' sowie die unbefristete ,Niederlassungserlaubnis', an deren Ende eine Einbürgerung stehen kann." (Schneider 2007a). Beide Aufenthaltstitel sind an die Motive und Qualifikationen der Zuwander_innen geknüpft und die Anforderungen, die zum Erhalt der Niederlassungserlaubnis erfüllt sein müssen, sind hoch. So ist der Erhalt der Aufenthaltserlaubnis aufgrund von „Ausbildung (z.B. Studierende), Erwerbstätigkeit (ausländische Arbeitnehmer), humanitären Gründen (z.B. Flüchtlinge und Asylsuchende) sowie familiären Motiven (Familiennachzug)“ (ebd.) möglich, während die unbefristete Niederlassungserlaubnis an folgende Bedingungen gebunden ist: „Sicherung des Lebensunterhalts und angemessenen Wohnraums für die eigene Familie, Nachweis von Beiträgen zur Altersvorsorge, Straffreiheit, ausreichend deutsche Sprachkenntnisse, Erlaubnis zur Erwerbstätigkeit, mindestens fünfjähriger vorheriger Besitz einer Aufenthaltserlaubnis“ (ebd.). 2007 wurde dieses Gesetz überarbeitet und an EU-Richtlinien angepasst (Meier-Braun 2013, S. 20). Die Veränderungen brachten auch schärfere Gesetze mit sich, die besonders die Einreise von Menschen aus Nicht-EU-Ländern einschränkte (ebd.). Diese stießen auf viel Kritik von vor allem türkischen Migrant_innenvereinen und Organisationen, die im Bereich Migration und Flüchtlinge tätig waren. So wurde damals im Bleiberecht beschlossen, dass Menschen, die langjährig geduldet ${ }^{11}$ waren, nach sechs (Haushalt mit Kindern) beziehungsweise acht Jahren (Haushalt ohne Kinder) Bleiberecht erhalten sollten (Schneider 2007b). Entscheidend sind jedoch nicht allein die Aufenthaltsdauer in Deutschland, sondern noch etliche weitere Bestimmungen (wie zum Beispiel der Nachweis von ausreichendem Wohnraum, Deutschkenntnissen sowie weitgehende Straffreiheit). widerrufen werden kann (Vgl. Diakonie (2014): Bleiberecht auf einen Blick. 
Sind diese Voraussetzungen erfüllt, erhalten sie eine Aufenthaltserlaubnis „auf Probe“ (ebd.), die nur verlängert wird, wenn die Person oder Familie ihren Lebensunterhalt durch Erwerbstätigkeit verdient. Geduldete Menschen dürfen sich nach einem neunmonatigen Aufenthalt in Deutschland eine Arbeit suchen, wobei sich die Suche für geduldete Flüchtlinge schwierig gestaltet, da häufig Deutsche, EU-Bürger und Menschen mit Aufenthaltsgenehmigung von den Arbeitgeber innen bevorzugt werden (Pro Asyl (2014).

Die Felder Integration, Einbürgerung, Familiennachzug enthalten Regelungen, die damals wie heute umstritten sind (Meier-Braun 2013, S. 20). Laut Migrant_innenvereinen und anderen Initiativen tragen Regelungen wie beispielsweise das Optionsmodell oder die Regelungen zum Familiennachzug nicht zur Integration bei, sondern fördern vielmehr die Ausgrenzung eingewanderter oder geflohener Personen und Familien (ebd., S.18). Das Optionsmodell sieht vor, dass ,sich in Deutschland geborene Kinder ausländischer Eltern, die die deutsche Staatsangehörigkeit erhalten haben, mit der Volljährigkeit für die deutsche oder die ausländische Staatsangehörigkeit ihrer Eltern entscheiden müssen" (ebd., S.18). Von dieser Regelung sind nur Kinder betroffen, deren Eltern aus Nicht-EU-Ländern stammen, während Kinder von Eltern aus EU-Staaten die doppelte Staatsbürgerschaft behalten dürfen (ebd.). Die Regelungen zum Familiennachzug sehen vor, dass die Ehepartner_innen aus Nicht-EU-Ländern nur nachkommen dürfen, „wenn sie volljährig sind und bereits vor der Einreise einfache Deutschkenntnisse nachweisen können“" (ebd., S.20), eine Regelung, die jedoch nicht für Staaten gilt, deren Bürger visumsfrei nach Deutschland einreisen dürfen (wie die USA, Südkorea, Japan und Australien). Migrant_innenvereine und andere Institutionen sehen in dieser Regelung eine eindeutige Diskriminierung von Bevölkerungsgruppen aus Regionen wie dem Nahen und Mittleren Osten und aus afrikanischen Staaten (Schneider 2007a). Eine Kritik, die auch von politischen Parteien geteilt wird, die diese Regelung nicht nur ,als menschenrechts- und grundrechtswidrig, sondern auch als frauen-, familien- und integrationsfeindlich" (Schneider 2007) einstufen.

Die Asyl- und Flüchtlingspolitik steht auch nach den Neuwahlen im Jahr 2013 im Fokus der Migrationspolitik Deutschlands (Rebeggiani 2013). Nach der Bundestagswahl beschloss die große Koalition weitere Veränderungen: Danach soll die Residenzpflicht für geduldete Flüchtlinge auf das gesamte Bundesland ausgeweitet werden, so dass es dieser Personengruppe möglich ist, sich in ganz Deutschland zu bewegen. Außerdem soll es asylsuchenden und geduldeten Menschen nach drei Monaten Aufenthalt in Deutschland möglich gemacht werden, sich auf dem freien Arbeitsmarkt um eine Arbeitsstelle zu bewerben. Neben weiteren Lockerungen (zum Beispiel der Erlaubnis eines früheren Beginn der Deutschkurse) für Asylbewerber_innen und Flüchtlinge, werden die angedachten Neuregelungen auch Einschränkungen enthalten, wie beispielsweise die Erklärung der Länder Mazedonien, Bosni- 
en-Herzegowina und Serbien zu ,sicheren Herkunftsländern“ (Rebeggiani 2013). Flüchtlingsorganisationen kritisieren diesen Vorschlag, da es eine Entscheidung wäre, die sich besonders negativ auf die Bevölkerungsgruppe der Roma auswirken würde, eine Bevölkerungsgruppe, die in ihren Herkunftsländern stark von Armut und Diskriminierung betroffen sei (ebd.). Trotz vielseitiger Kritik wurden die neuen Regelungen im September 2014 verabschiedet, was zur Folge hat, dass die Asylanträge der Menschen aus genannten Ländern nicht mehr individuell geprüft, sondern direkt abgewiesen werden (Preuß 2014).

\subsection{Zur Migrationspolitik der Schweiz}

Ähnlich wie in Deutschland wurde auch in der Schweiz lange Zeit die Meinung vertreten, kein Einwanderungsland zu sein, obwohl sich die Entwicklung zum Einwanderungsland bereits um die Jahrhundertwende (vom 19. zum 20. Jahrhundert) aufgrund der Industrialisierung abzeichnete. Anfangs verfolgte die schweizerische Politik eine liberale Migrationspolitik, die sich jedoch in den 1930er Jahren zu einer restriktiven Migrationspolitik wandelte (Carell 2013). Zu diesem Zeitpunkt wurde ein Gesetz erlassen, das bis 2008 die gesetzliche Grundlage der schweizerischen Migrationspolitik bleiben sollte. In diesem wurden Einwander_innen in drei Kategorien eingeteilt: „Saisonarbeiter_innen, Jahresaufenthalter_innen und Niedergelassene“" (ebd.). Eng an die Aufenthaltsgenehmigung gekoppelt war die Arbeitserlaubnis, die nur erteilt wurde, wenn schweizerische Arbeitskräfte fehlten. Obwohl die Schweiz nach dem Zweiten Weltkrieg einen wirtschaftlichen Aufschwung erlebte und auf zusätzliche Arbeitskräfte aus dem Ausland angewiesen war, wurde die Migrationspolitik in den 1960er Jahren weiter verschärft. Die Stimmen aus der schweizerischen Bevölkerung, die sich deutlich gegen vermeintliche Überfremdung ihres Landes aussprachen, nahmen zu, was sich politisch in weiteren Einschränkungen für Einwander_innen niederschlug (ebd.). Nachdem sich die schweizerische Wirtschaft in den 1980er Jahren von der Ölkrise erholt hatte, wurde das Land erneut interessant für Einwander_innen. Diejenigen, die bis zu diesem Zeitpunkt über eine Aufenthaltsgenehmigung verfügten, erhielten Zugang zu staatlichen Leistungen, was sie veranlasste $\mathrm{zu}$ bleiben. Sonst aber wurde die Einwanderung mithilfe des „Drei-Kreis-Modells“ (ebd.) streng geregelt: Zum ersten Kreis zählten Bürger innen aus der Europäischen Union und aus Staaten, die der Europäischen Freihandelsassoziation (EFTA) zugehörig waren. Zum zweiten Kreis zählten all die Länder, „bei denen eine ,kulturelle Nähe` zur Schweiz, solide Handels- und Migrationsbeziehungen, sowie die Einhaltung der Menschenrechte konstatiert wurden (z.B. Kanada, USA, mittel- und osteuropäische Staaten)“ 
(Carell 2013). Einwanderung aus dem dritten Kreis, also von denjenigen Menschen, die eine vermeintliche ,kulturelle Distanz' zur Schweiz haben, wurde nur in Ausnahmefällen genehmigt, wenn nämlich der/die Einwander in aufgrund von hoher Qualifizierung in einem bestimmten Sektor nützlich für die Schweiz war (ebd.). Das Modell wurde aufgrund des Diskurses zur angeblichen ,Überfremdung ' der Schweiz kritisiert und 1998 schließlich verändert. Dennoch schürte der Diskurs über angebliche, Überfremdung ' die Ängste der Bevölkerung davor, dass zu viele Einwander_innen aus fremden ,Kulturen` die Schweizer ,Kultur' verwässern könnten. Das Modell wurde umgewandelt in ein System, das weiterhin die Bürger_innen aus der Europäischen Union und den EFTA-Staaten bevorzugte, während Bürger_innen anderer Staaten meist nur einwandern durften, wenn sie hoch qualifiziert waren. Diese Regelung wurde 2008 erneut gesetzlich bestätigt und gilt bis heute. Der Volksentscheid zur Beschränkung von Zuwanderung von 2014 verdeutlicht, dass der Diskurs vor der vermeintlichen, Überfremdung' der Schweiz Wirkung zeigt. Die Schweizer haben sich in diesem zwar knapp, aber dennoch dafür ausgesprochen, Zuwanderung weiter zu beschränken. Danach wird Einwanderung nur genehmigt, wenn die wirtschaftliche Lage dies nötig macht. Auch die schweizerische Flüchtlings- und Asylpolitik ist im Laufe der Jahre deutlich restriktiver geworden. Seit 2006 gelten strenge Gesetze, die Asylbewerber_innen bei Nichterfüllung bestimmter Kriterien mit Strafen wie beispielsweise Ausschluss von der Sozialhilfe belegen (Carell 2012).

\subsection{Zur Migrationspolitik Englands}

In England herrschte bis in die 1960er Jahre eine Politik der offenen Grenzen. Angehörige der Commonwealth-Staaten ${ }^{12}$ durften in das Vereinigte Königreich einreisen und sich dort aufhalten (Hansen 2007). Im Zuge dieser Vereinbarung kamen besonders viele allein stehende junge Männer, die im Verlauf der Jahre Gebrauch von der Regelung des Familiennachzugs machten, was die Zahl der Einwander_innen im Land stetig vermehrte (ebd.). Der wirtschaftliche Aufschwung, der sich nach dem Zweiten Weltkrieg in England einstellte und einen Anstieg an Arbeitsstellen mit sich brachte, zog weitere Menschen an, besonders aus den ehemaligen britischen Kolonien (vgl. Hansen 2007a). Im Jahr 1962 beschloss die damalige britische Regierung erstmals die Einwanderung zu reglementieren, ein Beschluss, der von der damaligen Opposition ,vehement als populistische und rassistische Maß-

12 Dabei handelt es sich um eine Verbindung aus den Staaten des Vereinigten Königsreichs Großbritannien und Nordirland und Staaten der ehemaligen Kolonien. 
nahmen angeprangert" (ebd.) wurde. Nach dem Regierungswechsel im Jahr 1965 sah sich auch die neugewählte liberale Regierung gezwungen, Maßnahmen zur Regulierung der Einwanderung zu erlassen, mit der Begründung, dass durch den Familiennachzug eine zu hohe Zahl weiterer Einwander_innen ins Land käme. Die Stimmung innerhalb der Bevölkerung, die die Einwander_innen zunehmend als Konkurrenz auf dem Arbeitsmarkt ansah und außerdem um den Verlust „britische[r] Werte“ (ebd.) fürchtete, trug zu dem politischen Kurswechsel bei. Trotz des Beschlusses, Einwanderung künftig stärker zu reglementieren, versuchte die Regierung zusammen mit öffentlichen Institutionen und Medien, Rassismus entgegenzutreten, indem Schritte eingeleitet wurden „Minderheiten“ (Hansen 2007a) als Bestandteil der britischen Bevölkerung sichtbar zu machen. Dennoch wurde eine einwanderungsbeschränkende Politik verfolgt, die sich dahingehend veränderte, dass der Status des Asylbewerbers eingeführt wurde, womit eine Spezifizierung des/der allgemeinen Einwanderers/Einwanderin von damals durchgesetzt wurde. Diese Spezifizierung zielt darauf ab, die Zahl der Asylbewerber_innen zu dezimieren, was letztlich auch gelang (Hansen 2007b) Im Jahr 2002 erfolgte ein Bruch in der britischen Migrationspolitik, als der Beschluss, Einwanderung gezielt zu steuern, mit einem Plan durchgesetzt wurde, der das Ziel hatte, qualifizierte Einwanderung zu fördern. Es wurde ein Punktesystem entwickelt, das sowohl am Arbeitsmarkt wie auch an den Qualifikationen des/der Einwanderers/in orientiert Einwanderung regelt (ebd.). Das Punktesystem bietet den hoch qualifizierten Einwander innen die besten Einwanderungsbedingungen, da deren Einreise unabhängig von einer Arbeitsstelle ist. Im Punktesystem weit oben sind außerdem Einwander_innen, die eine berufliche Qualifikation mitbringen, die in England benötigt wird. Einwander_innen mit geringer Qualifikation dürfen einreisen, wenn sie eine Arbeitsstelle in England nachweisen können, während Studierende oder Einwander_innen, deren Aufenthalt von Anfang an begrenzt ist, auf den beiden unteren Stufen des Systems eingeordnet werden (ebd.) Das System schränkt die Einwanderung für diejenigen Einwander_innen am stärksten ein, die nicht der Europäischen Union zugehörig sind und nur über geringe berufliche Qualifikationen verfügen. Kombiniert mit dem politischen Beschluss, die Grenzen Englands stärker zu kontrollieren, kommen weitere repressive Maßnahmen gegen ungewollte Einwander_innen zum Einsatz, wie beispielsweise der digitale Fingerabdruck (Hansen 2007b).

In England beherrschte besonders um die Jahrtausendwende der Begriff „Multikulturalismus“ die Gesellschaft und Politik (Hansen 2007c), ein Begriff, der nie genau definiert wurde, jedoch lange Zeit den politischen und medialen Diskurs dominierte (ebd.). Nach den Auseinandersetzungen zwischen Angehörigen asiatischer Einwander_innen und weißen, veränderte sich die Diskussion um den so genannten ,Multikulturalismus'. Der Politikwissenschaftler Hansen glaubt jedoch nicht, dass der britische ,Multikulturalis- 
mus ${ }^{`}$ aufgrund der Ereignisse und daraus entstandene Diskurse abgeschafft sei. Seiner Meinung nach zeichne sich der ,Multikulturalismus“ in England durch drei Grundbestandteile aus:

„Erstens steht er für die Anerkennung der gesellschaftlichen Realität des Vereinigten Königreichs als Gesellschaft, die aus vielen Kulturen besteht. Zweitens basiert er auf der weit verbreiteten Ansicht, dass Multikulturalismus eine ,gute Sache' sei. Drittens liegt ihm das Prinzip zugrunde, dass ,britisch werden' nicht bedeutetet, dass Menschen ihre früheren kulturellen Bindungen und Praktiken ablegen müssen." (ebd.).

Auf Basis dieser drei Elemente gestalte sich das gesellschaftliche Leben in Großbritannien auch heute.

\subsection{Zur Migrationspolitik Italiens}

Italien erlebte einen Wandel vom Auswanderungs- zum Einwanderungsland, ein Wandel, auf den Anfang der 1970er Jahre zunächst nicht reglementierend eingewirkt wurde. In den letzten 30 Jahren stieg die Einwanderungszahl stetig an und ist heute so hoch wie in keinem anderen Land der Europäischen Union (Carell 2012) Besonders viele Menschen kommen aus Osteuropa, vorwiegend Albanien und Rumänien (ebd.). Der stetige Anstieg der zuwandernden Bevölkerungsgruppen wirkt sich auf die Migrationspolitik Italiens aus und versucht die steigenden Einwanderungszahlen mithilfe von Einwanderungsgesetzen zu steuern (Di Muzio 2012). Das Einwanderungsgesetz von 1990, in dem eine Visapflicht für die Hauptherkunftsländer eingeführt und eine gesetzlich geregelte Einwanderungsquote festgelegt wurde, sollte Einwanderung zusätzlich durch vermehrte Abschiebungen regeln (ebd.). Das Gesetz erzielte jedoch nicht den erwünschten Erfolg. Die Zahl der Einwander_innen stieg weiter an, weshalb es erweitert wurde. Die Erweiterung bestand darin, ausgewanderten italienischen Nachkommen die Rückkehr und das Erlangen der italienischen Staatsbürgerschaft zu erleichtern. Gleichzeitig wurde es für alle Einwander_innen aus Nicht-EU Ländern erschwert, einen italienischen Pass zu erhalten. Ende der1990er Jahre wurde erneut ein Gesetz erlassen, dass die so genannte illegale Einwanderung einschränken sollte, z. B. durch die Rückführung illegaler Migrant_innen in ihre Heimatländer (bereits auf See) oder die Inhaftnahme illegal Eingereister bis zur Abschiebung (ebd.). Im selben Gesetz wurde verankert, dass alle legal eingereisten Migrant_innen Zugang zum italienischen Bildungs- und Gesundheitssystem erhalten und nach fünfjährigem Aufenthalt die unbefristete Aufenthaltserlaubnis beantragt werden kann. Anfang 2000 und unter Berlusconi wurde dieses 
so genannte Bossi-Fini-Gesetz modifiziert und so genannte illegale Einwanderung strenger geregelt sowie die Zahl legaler Einwanderung herabgesetzt (Di Muzio 2012). Besonders die Regelung, dass bereits auf dem Meer aufgegriffene Schiffe überprüft werden sollen, mit dem Ziel, Asylberechtigte von illegalen Einwander_innen zu trennen, sorgte für internationale Kritik. Menschenrechtler_innen befürchten eine fehlerhafte Prüfung mit der Folge der Zurückweisung asylberechtigter Personen, deren Leben im Heimatland bedroht sei. Des Weiteren wird Migration so ,als soziale Gefahr deklariert, die eingedämmt werden muss, selbst wenn dabei die Rechte von Zuwanderern gravierend beschnitten werden" (ebd.). Obwohl auch dieses strenge Gesetz die illegalen Einwanderungen nicht minimieren kann, wird größtenteils daran festgehalten. Trotz des Bossi-Fini-Gesetzes kommen Flüchtlinge und Asylsuchende weiter nach Italien, meist über das Meer. Viele Menschenrechtorganisationen betrachten den Versuch der Eingrenzung weiterer Flüchtlinge über den Seeweg und die damit verbundene vertragliche Einigung Italiens mit Libyen für heikel. In dieser wird Libyen dazu verpflichtet, vermehrt die eigenen Grenzen zu kontrollieren, um die Zahl afrikanischer Flüchtlinge nach Italien zu begrenzen. Gleichzeitig ist Lybien aber ein Land, das die Genfer Flüchtlingsrechtskonvention nicht unterzeichnet hat (Di Muzio 2012), was die Befürchtung mit sich bringt, dass die Menschenrechte der Flüchtlinge nicht gewahrt werden. Die aktuellen Entwicklungen zeigen, dass weder verschärfte Gesetze noch vermehrte Grenzsicherungen Flüchtlinge davon abhalten, den gefährlichen Seeweg nach Italien (und in andere EU-Länder) anzutreten. Nach Angaben der Internationalen Organisation für Migration haben fast 45000 Menschen versucht, das Meer von Afrika nach Malta oder Lampedusa zu überqueren. Circa 700 ertranken bei diesem Versuch (Zeit Online 2014). Nach den schweren Bootsunglücken 2013 vor Lampedusa, bei denen hunderte Flüchtlinge ertranken, sind die Küstenwachen der betroffenen Staaten der EU und Frontex ${ }^{13}$ laut EU-Recht dazu verpflichtet, Flüchtlingen in Seenot zu helfen, was bislang eine freiwillige Entscheidung darstellte (Rebeggiani 2014). Des Weiteren dürfen Flüchtlinge an den EU-Außengrenzen nicht mehr zurückgedrängt oder zur Rückkehr gezwungen werden. Menschen, die in Seenot geratenen Flüchtlingsbooten helfen, dürfen nicht mehr bestraft werden. Die Boote müssen weiter daraufhin überprüft werden, ob besonders schutzbedürftige Personen an Bord sind, wie beispielsweise unbegleitete minderjährige Flüchtlinge, denen besondere Rechte zustehen (ebd.) Trotz der neuen Regelungen steht Italien vor der Herausforderung, die Flüchtlinge, die an Italiens Küsten ankommen, zu versorgen und die italieni-

13 Frontex ist ,eine Agentur der Europäischen Union und wurde zum 1.5.2005 [...] gegründet. Frontex soll dazu beitragen, die Außengrenzen der EU zu schützen. Frontex beschäftigt Vertragsbedienstete und Entsandte von Behörden der Mitgliedstaaten." Es ist keine Polizei, sondern eine Stelle zur Koordination und Unterstützung der nationalen Grenzpolizei operierenden Stelle. (Bundeszentrale für politische Aufklärung 2014: Frontex.). 
sche Küstenwache vor der Aufgabe, die Flüchtlingsboote in Seenot zu retten, um zu verhindern, dass weitere Flüchtlinge ertrinken. Doch einmal in Italien angekommen, erwartet die Flüchtlinge ein schwieriges Leben. Laut einem Bericht von Amnesty International leben allein in Rom ,mehr als 2000 Flüchtlinge in besetzten Häusern, davon ca. 1400 bis 1500 Eritreer, Somalis und Äthiopier" (Schwarz 2013). Ein fehlender fester Wohnsitz hat für Flüchtlinge weitreichende Konsequenzen, da sie ohne diesen nur schwer einen Arbeitsplatz bekommen und von der medizinischen Versorgung ausgeschlossen bleiben, da zur Beantragung einer so genannten "Gesundheitskarte“ (ebd.) ebenfalls der Nachweis eines festen Wohnsitzes verlangt wird. Die Situation der Flüchtlinge oder Asylsuchenden in Italien wird zunehmend schwierig, da die Anzahl der Flüchtenden steigt. Seit Anfang des Jahres 2014 sind 50000 Migrant_innen nach Italien geflohen, die meisten kommen aus Syrien und fliehen vor dem Bürgerkrieg (Zeit Online 2014). Im letzten Jahr sind laut diesem Bericht 43000 Flüchtlinge in Italien angekommen. Die Zahlen verdeutlichen, dass die Menschen, sei es aufgrund von Krieg, politischer Verfolgung oder Armut, ihre Heimatländer verlassen und trotz der restriktiven Migrationspolitik der Europäischen Union einen Weg finden, nach Europa zu gelangen.

\subsection{Schlussfolgerungen}

Zusammenfassend kann festgehalten werden, dass sich die Migrationspolitik der Einwanderungsländer dadurch auszeichnet, Migration zu beschränken, mit dem Ziel nur die Einwander_innen in das Land zu lassen, die aufgrund ihrer beruflichen Qualifikationen oder ihrer finanziell gesicherten Lage dem Einwanderungsland von Vorteil sind (Oltmer 2013b).

Durch Abschiebehaft von so genannten illegalen Einwander_innen wird Migration kriminalisiert. Aufgrund der Entwicklungen der vergangenen Jahrzehnte findet sich der Begriff, Migrationsregime' zur Benennung oder Kennzeichnung der Systeme zunehmend wieder, da in diesem die ,asymmetrische[n] Macht-Verhältnis[se]“ (Tsianos 2010, S. 2), durch die die Migrationspolitik geprägt ist, zum Ausdruck kommen. Unter ,Migrationsregimen“ werden „Sphären des Aushandelns von Migration“ (Netzwerk Flüchtlingsforschung 2013) gefasst und auf das wechselseitige Verhältnis zwischen Migration und Nationalstaaten hingewiesen (Oltmer 2013a, S. 34). ,Migrationsregime“ beeinflussen „die Umsetzung und Gestaltung von Migrationsoptionen“ (ebd.) und beschränken auf diese Weise Einwanderung unter ökonomischen Gesichtspunkten. Die Beeinflussung der Migration erfolgt somit durch ,,individuelle[ ], kollektive[ ] und institutionelle[ ] Akteure" (ebd.) und wird durch unterschiedliche „Interessen und Zielen“ (ebd.) gelenkt. Diejenigen, die die 
Regeln aushandeln und durchsetzen, sind denjenigen, die dadurch begrenzt werden „maßlos überlegen“ (Tsianos 2010, S. 2). Die Wirtschaftsmächte der Welt sorgen durch repressive Maßnahmen dafür, Einwanderung nach ihren Regeln zu kontrollieren. Trotz der repressiven Maßnahmen an den Außengrenzen der EU kann Zuwanderung aber nicht gänzlich verhindert werden, denn Flüchtlinge werden auch in Zukunft Wege finden, um aus den politischen oder sozialen Verhältnissen ihrer Heimat zu fliehen (Oltmer 2013b). Die in den Einwanderungsländern geführten Diskurse über Migration sorgen dafür, Migrant_innen als Belastung für die Sozialsysteme zu konstruieren, die in Form immenser ,Flüchtlingswellen' über Europa hereinbrechen. Diskurse dieser Art homogenisieren ganze Gruppen und stellen diese als potentielle Bedrohung für die jeweilige Mehrheitsgesellschaft dar, was sich beispielsweise in der Debatte um die Zuwanderung von Menschen aus Rumänien oder Bulgarien im Jahr der Wahlen 2013 in Deutschland gezeigt hat. In dieser wurden Menschen aus genannten Herkunftsstaaten generalisierend unter den Verdacht gestellt, nach Deutschland kommen zu wollen, um den deutschen Sozialstaat auszunutzen. In den Hintergrund rückte der differenzierte Blick, der zeigte, dass die Debatte nicht nur realitätsfern war, was die Zahlen belegen, sondern auch, dass es sich um eine diskriminierende Debatte handelte, die zur Ausgrenzung ganzer Bevölkerungsgruppen (Meier-Braun 2013, S. 15) beitrug. 


\section{Theoretische Perspektiven}

Im folgenden Kapitel wird der Blick auf unterschiedliche theoretische Perspektiven gerichtet, denen im Kontext dieser Untersuchung zu weiblicher Genitalbeschneidung und beschnittenen Migrantinnen in Europa eine wichtige Rolle zukommt. Dieser Teil der Arbeit dient nicht allein dazu, die verschiedenen theoretischen Perspektiven aufzuzeigen, aus denen heraus das Phänomen genitaler Beschneidung betrachtet werden kann. Es bildet auch die theoretische Grundlage, die für die Analyse der durchgeführten qualitativen Interviews mit beschnittenen somalischen Migrant_innen notwendig ist, da sich die jeweiligen Erkenntnisse der nachfolgend skizzierten Perspektiven in den Aussagen widerspiegeln. Abschließend wird am Ende dieses Kapitels noch auf die Konstruktion von ,Kultur' eingegangen. Dieser Blick erscheint vor dem Hintergrund, dass weibliche Genitalbeschneidung häufig als Teil der ,Kultur' bestimmter Bevölkerungsgruppen beschrieben wird, besonders wichtig und folgt der Frage, was unter ,Kultur' verstanden wird.

\subsection{Postkoloniale Theorien}

Die Postkolonialen Studien präsentieren sich als „komplexes, zuweilen diffuses Theoriefeld“ (Reuter/Villa 2010, S. 16). Seit drei Jahrzehnten existieren postkoloniale Diskurse, das Wesen dieser "Theorierichtung“ (Castro Varela/Dhawan 2010, S. 304) ist aus Sicht Castro Varelas und Dhawans heute allerdings besonders schwer auszumachen. Bei den postkolonialen Studien handelt es sich nicht um eine Theorie, da sie sich mehr aus ,verschiedene[n] Beiträgen mit unterschiedlichen theoretischen und analytischen Ansätzen“" (Reuter/Villa 2010, S. 16) zusammensetzt. Da sie keiner spezifischen Fachrichtung zuzuordnen sind, kann der postkoloniale Blickwinkel auf alle Fachrichtungen angewandt werden und ist sowohl als ,Verfahren wie auch [...] [als] Perspektive" (ebd.) zu verstehen.

Der Begriff ,postkolonial“ ist nicht eindeutig definierbar, er bleibt „trotz aller Versuche der Klärung unscharf und heiß debattiert" (Castro Varela/Dhawan 2005, S. 23). Je nachdem zu welcher Zeit der Begriff verwendet wird, fokussiert er unterschiedliche Phänomene, die sich aus dem Kolonialismus ergeben haben. Somit ist das Präfix ,post ${ }^{\star}$ nicht im wörtlichen Sinne als ,danach', also die Zeit nach der Unabhängigkeit kolonisierter Länder zu lesen. Postkolonialismus ist ,eher als eine politisch motivierte Analysekategorie zu verstehen, die einerseits die nachhaltige Prägung der globalen Situa- 
tion durch Kolonialismus, Dekolonialisierung und neokolonialistische Tendenzen aufzeigt“" (Reuter/Villa 2010, S. 17).

Die Kolonialisierung ist eine Geschichte, die nicht durch einen Anfangsoder Endpunkt bestimmt werden kann, sondern sich bis in die heutige Zeit zieht, da immer wieder neue Wege gefunden werden, an die Ressourcen (Arbeitskräfte wie Rohstoffe) verschiedener Länder zu kommen, was mithilfe bestimmter Unterdrückungsformen durchgesetzt wird (Castro Varela/Dhawan 2005, S. 24). Bei der Dekonstruktion kolonialer Geschichte betonen die Autor_innen außerdem, dass die beidseitige Verstrickung kolonisierter Länder mit den Kolonialländern zu beachten ist. Mithilfe postkolonialer Theorien wird ,der Imperialismus als ein europäisches wie außereuropäisches Gesamtphänomen“" (ebd.) untersucht. Die eng mit den Prozessen der Kolonisierung verbundenen Macht- und Herrschaftsstrukturen, die sich hinter kolonialen Diskursen verstecken, stehen bei der Analyse der Geschichtsschreibung im Mittelpunkt. Mithilfe „koloniale[r] Diskursanalyse“ (Castro Varela/Dhawan S. 24) als ein wichtiges Instrument bei der Dekonstruktion wird koloniale Geschichte neu gelesen. Zusammenfassend kann gesagt werden, dass „die Postkolonialen Studien einen Schnittpunkt zwischen den Debatten über race, Kolonialismus, class, gender, Politik und Sprache" (Ashcroft/Griffith 2008, S. 2) bilden.

Ein anschauliches Beispiel dafür, wie Diskurse zur Kolonialisierung beitragen, liefern die Analysen Edward M. Saids, der als einer der Vordenker postkolonialer Studien gilt. In Anlehnung an Foucault geht Said von Machtund Hierarchieverhältnissen aus, die gesellschaftliche und wissenschaftliche Diskurse leiten und am Ende ein bestimmtes Bild des untersuchten Phänomens konstruieren. In seiner Studie „Orientalism“ (1978), „die mittlerweile als ,Gründungstext" Postkolonialer Literatur" (Reuter/Villa 2010, S. 20) gilt, verdeutlicht er anschaulich wie sich, der Orient" aus einer Konstruktion Europas beziehungsweise europäischer Forschungen formt (Castro Varela/Dhawan 2005, S. 30). Dieses vermeintlich objektive Bild des Orients wird aus Sicht so genannter, Orientexperten', aus europäischem Blickwinkel heraus konstruiert, verwissenschaftlicht und am Ende als ,Wahrheit' verbreitet. Said zeigt anhand dieses Beispiels, wie mithilfe dieser machtvollen Diskurse die hegemonialen europäischen ,Kulturen ' für die Herabstufung der orientalischen Welt sorgen würden und dadurch die Trennung zwischen Orient und Europa vorantreiben und somit die Kolonisierung des Orients zu vereinfachen (Castro Varela/Dhawan 2005, S. 34).

In den Forschungen Spivaks, ebenfalls eine Vordenkerin Postkolonialer Studien, wiederum steht der Aspekt, gender', ,class ${ }^{6}$ und ,race' im Mittelpunkt ihrer Forschungen. Sie legt dabei den Fokus auf so genannte ,subalterne ${ }^{614}$ Frauen (Castro Varela/Dhawan 2005, S. 56). Aus Spivaks Sicht werden 
,subalterne' Frauen in zweierlei Hinsicht ausgebeutet und unterdrückt, da sie einerseits vom Imperialismus und der Globalisierung wirtschaftlich ausgebeutet werden und andererseits die Unterordnung in ein patriarchal geprägtes Gesellschaftssystem erfahren, welches die Unterdrückung ihrer Rechte mit sich bringe (ebd., S. 58). Wo Said in Anlehnung an Foucault die Befreiung der unterdrückten Masse für möglich hält, betrachtet Spivak die ,subalternen Frauen als Gefangene dieser unterdrückenden Strukturen, da sie weder politisch organisiert sind noch einer hegemonialen Klasse angehören. Am Beispiel der Witwenverbrennung macht Spivak dies deutlich. Laut Spivak würden Männer mit ,Traditionen' wie der Witwenverbrennung Frauen für den Erhalt ihrer patriarchalen Strukturen instrumentalisieren, indem sie ihnen die Aufgabe übertragen, diese ,Tradition' an die nächste Generation weiterzugeben. Gleichzeitig diene die ,Tradition" der Witwenverbrennung im ,westlich" geprägten feministischen Diskurs als das Beispiel für Frauenunterdrückung. Einer indischen Frau, die mit dieser ,Tradition' lebt, sei es nicht möglich, sich unabhängig dieser beiden entgegen gesetzten Positionen zu positionieren. Spricht sie sich für Witwenverbrennung aus, sorgt sie für den Erhalt des patriarchal geprägten Systems, spricht sie sich dagegen aus, ordnet sie sich in den ,westlich“ geprägten feministischen Diskurs ein, der mit dem Argument, die unterdrückten Frauen Indiens retten zu müssen, die (teilweise gewaltsame) Einmischung des ,Westens' legitimiert, um den Frauen eine Entwicklung nach, westlichem “ Beispiel zu ermöglichen. Witwenverbrennung würde auf diese Weise von , westlichen' Feminist innen nicht nur als Beispiel für die Unterdrückung indischer Frauen benutzt, sie würde gleichzeitig als Beispiel für die Rückständigkeit eines Landes herangezogen. Aus Spivaks Sicht seien ,subalterne' Frauen daher zum Schweigen verbannt, da ihre Stimme nicht gehört, sondern einem der beiden Systeme zugeordnet würden. Ihre Äußerungen würden stattdessen aufgegriffen und dafür benutzt, die eigene Position zu legitimieren, während die Frauen selbst in diesem Prozess in den Hintergrund rückten.

Dieses Phänomen ist auf beschnittene Frauen übertragbar. Die vermeintliche Solidarität, westlicher' Feminist_innen mit beschnittenen Frauen ist ebenso fragwürdig, da auch der Diskurs über beschnittene Frauen lange Zeit davon geprägt war, betroffenen Frauen jegliche Handlungskompetenz und Entscheidungsfreiheit abzusprechen und sie als Opfer zu stigmatisieren (Levin/Schwarzbauer 1979, S. 23). Ähnlich der Witwenverbrennung oder der teilweise sehr undifferenzierten Debatte um das Kopftuch, wird genitale Beschneidung von manchen, westlichen' Feminist_innen zu dem Zeichen von Unterdrückung schlechthin erhoben, was sich teilweise in der Literatur

,Subaltern' nennt er die, die keiner hegemonialen Klasse angehören, die politische unorganisiert sind und über kein allgemeines Klassenbewusstsein verfügen" (Castro Varela/Dhawan 2005, S. 69). Diejenigen also, die gesellschaftlich nicht vertreten werden, die eher im ländlichen Raum zu finden sind. 
niederschlägt. Ein Prozess, der Betroffene zu Stimmlosen macht und von der Konstruktion der ,Anderen' lebt.

Die Konstruktion der ,Anderen', das so genannte ,Othering', ist ebenfalls ein Begriff, der von Spivak (1985) geprägt wurde und der im Kontext dieser Arbeit von Bedeutung ist. Spivak beschreibt damit den im kolonialen Diskurs zu beobachtenden Prozess der Abgrenzung von ,Anderen' als Notwendigkeit zur Konstruktion der eigenen Normalität (Castro Varela/Dhawan, S. 60). Somit werden die ,Anderen' erst durch diese kolonialen Diskurse konstruiert, in denen sich Machtpositionen derjenigen widerspiegeln, die diese Diskurse anführen. Anhand von Kategorien wie Religion, Nationalität oder Hautfarbe wird aus ,europäischer' Perspektive heraus ,fremd' konstruiert und in Abgrenzung zur eigenen Identität beschrieben. Die Auswirkungen zeigen sich in der bereits beschriebenen Studie Saids zur europäischen Konstruktion des ,Orients'. Das Sprechen über ,Andere' hat die Kolonialisierung bestimmter Völker durch die Europäer_innen ermöglicht, da diesem Prozess die Vorstellung zugrunde liegt, die ,Ānderen' seien weniger entwickelt. Es dient dazu, die eigene Vormachtstellung zu erhalten und auszubauen. An diese Gedanken anknüpfend, wird nachfolgend auf die Theorien der Kritischen Weißseinsforschung eingegangen, da diese die vorangegangenen Theorien und die damit verbundenen Erkenntnisse aus einer anderen Perspektive heraus ergänzen.

\subsection{Kritische Weißseinsforschung}

Die aus den postkolonialen Studien hervor gegangene Kritische Weißseinsforschung stellt im Kontext der Migration beschnittener Frauen nach Europa eine wichtige Perspektive dar. Diese dem angloamerikanischen Raum entsprungenen Studien basieren auf einem Anfang der 1990er Jahre vollzogenen Perspektivwechsel, der zur Folge hatte, dass nicht länger nur der Blick auf die von Rassismus betroffenen Menschen gerichtet wurde, sondern auf diejenigen, die diesen Rassismus hervorbringen und davon profitieren (Arndt 2006, S. 13). Das primäre Forschungsinteresse der Kritischen Weißseinsforschung liegt daher auf dem ,Weißsein', sowie den damit verbundenen hegemonialen Diskursen, die die Konstruktion bestimmter Kategorien hervorbringen. Dabei steht die Frage im Zentrum, wie Schwarze und ,People of Color von den bislang unmarkierten weißekonstruiert werden, mit welchen $\mathrm{Zu}-$ schreibungen diese Konstruktionen verbunden sind und welche Machtverhältnisse sich dahinter verbergen (ebd.). Da in diesem Zusammenhang der Begriff ,Rasse“ eine Rolle spielen wird, wird dieser nachfolgend in Kürze betrachtet.

Auch der Begriff ,Rasse“ wird als gesellschaftliche Konstruktion verstanden, die eng an die Definitionsmacht bestimmter gesellschaftlicher Gruppie- 
rungen geknüpft ist und zu keinem Zeitpunkt in der Geschichte als neutrale Beschreibung gedient hat (vgl. Jablonski 2014). Stattdessen dient die Konstruktion von ,Rasse', in der weißen eine vermeintlich natürliche überlegene Position zukommt, dazu, die Machtposition bestimmter Gruppierungen in der Welt zu legitimieren. Biologische Vorstellungen, wie beispielsweise der „Gedanke des Sozialdarwinismus, verstärkte[n] die Vorstellung noch, dass die Überlegenheit der weißen ,Rasse“ Teil der natürlichen Ordnung sei“ (Jablonski 2014). Arndt (2006) verweist darauf, dass „das Konzept ,Rasse'nicht auf den Menschen zu übertragen werden kann, da es keine reinerbigen Teilpopulationen gibt" (Arndt 2006, S.11). Vielmehr sind Menschen „Produkte komplexer genetischer Vermischung [...], die aus Migration und Durchmischung über tausenden von Jahren hervorgehen“" (Jablonski 2014) verschleiert. Mit wie viel Macht mit der Konstruktion von ,Rasse' einhergeht, zeigt sich an vielen historischen sowie gegenwärtigen Beispielen, in der die hierarchisch gegliederte Einteilung von Menschen dazu gedient hat beziehungsweise dazu dient, vermeintlich unterlegene Bevölkerungsgruppen zu unterdrücken und auf die im Verlauf dieser Ausführungen noch eingegangen wird.

Die Konstruktion von ,Rasse" wird zu einem Raster, durch das die Welt und die darin agierenden Subjekte gesehen, interpretiert und konstruiert werden (Arndt 2006, S. 11). Laut Arndt handelt es sich hierbei um eine Strukturkategorie, die lange Zeit in Stillschweigen gehüllt wurde. In Abgrenzung zu den Ideologien des Kolonialismus und des Nationalsozialismus wurde nach dem Zweiten Weltkrieg dazu übergegangen, Weißsein und die damit verbundenen Privilegien nicht mehr zu thematisieren. So wurde Weißsein „zur Selbstverständlichkeit, die nicht einmal mehr betont werden muss" (Arndt 2005, S. 27) und zu einer gesellschaftlichen Norm, aus der heraus ,die Anderen' betrachtet und konstruiert wurden, ohne dass sich die Konstruierenden selbst hinterfragen mussten. So sei Rassismus zu einem „Ordnungsprinzip“ (Eggers 2005, S. 57) geworden, das sich in vier eng miteinander verbundenen Stufen manifestiere. Auf der ersten Stufe würden aus Sicht der ,hegemonialen weißen Gruppe“ (ebd.) Schwarze und ,People of Color' in einem Gegensatz dargestellt, den weiße aus der Differenz zu sich selbst beschreiben und mit Zuschreibungen versehen. Aus weißer Perspektive würde auf diese Weise Wissen über die ,Anderen' geschaffen, das am Ende zu bestimmten Bildern über Schwarze und ,People of Color' führe. Die zweite Stufe diene laut Eggers dazu, die konstruierten Eigenschaften oder scheinbaren Wesensmerkmale zu naturalisieren und ,als unüberwindbare[n] Teil der ,Natur' von rassistisch markierten ,Anderen'[zu setzen]" (Eggers 2005, S. 58). Dieses Wissen über die ,Anderen' würde verbreitet und als natürliche Differenz zur weißen Gruppe dargestellt. Auf der dritten Stufe wird den ,Anderen' schließlich ein untergeordneten Platz innerhalb der weißen Gruppe zugewiesen, der auf der letzten Stufe schließlich „auf der Grundlage einer natürlich erscheinenden 
hierarchischen Ordnung erklärt werden [kann]“ (Eggers 2005, S. 58). Das hegemoniale weiße Zentrum kann somit unbenannt und unmarkiert bleiben, funktioniert dann sogar als eine neutrale Instanz" (ebd.), über die nicht debattiert werden muss.

Der Blick auf die Geschichte zeigt, dass die Kategorie des Weißseins lange Zeit als "naturgegebene" Norm und allen anderen "Rassen" überlegen" (Arndt 2005, S. 25) konstruiert wurde. Diese vermeintlich ,natürliche' Begründung der Überlegenheit weißer Menschen nicht-weißen Menschen gegenüber, geht mit einem Machtgefälle einher, das von den weißen zur Legitimation herangezogen wird, sich über andere Völker zu stellen, um diese nach europäischem Vorbild zu ,'erziehen“ und zu ,zivilisieren““ (ebd., S. 26). Die Annahme biologisch bedingter Überlegenheit erlaubte den weißen Kolonialmächten, sich weltweit auszubreiten und die vermeintlich ,unterlegene" SchwarzeBevölkerung zu unterdrücken. Bis weit ins 20. Jahrhundert hinein wurde das Bild des natürlich überlegenen weißen im europäischen Raum vertreten und zur Rechtfertigung von Gewalttaten an Schwarzen, Sklavenhandel, Ausbeutung und dergleichen herangezogen. Ein ganzes Jahrhundert lang wurden Einheimische ihrer Rechte und ihres Landes beraubt (ReedAndersson 2004). Afrika wurde zum ,homogene[n] und unterlegene[n] ,Andere[n]' und bedurfte daher der ,Zivilisierung' durch Europa“" (Arndt 2004). Konstruktionen, die sich mithilfe machtvoller Diskurse durchsetzen konnten und teilweise noch bis heute zu finden sind (ebd.). So wurde zu Kolonialzeiten in der Beschreibung afrikanischer Staaten oder Bevölkerungsgruppen Worte benutzt, die sich deutlich von der ,gehobenen", zivilisierten" Welt abhoben und zum Ziel hatten, die Bevölkerungsgruppen Afrikas herabzustufen (Arndt 2004). Ausgehend von einer Rassentheorie wurden die Menschen Afrikas beispielsweise als ,Neger', ,Mulatte' und ,Mischling' bezeichnet (ebd.). Allesamt handelt es sich um Bezeichnungen, die negativ konnotiert sind und die Unterlegenheit dieser ,Rasse' zum Ausdruck bringen sollen. So verweist Arndt beispielsweise darauf, dass ,ein Schwarzer Deutscher, nicht aber ein Kind aus einer weißen französisch-deutschen Beziehung als ,Mischling' bezeichnet" (Arndt 2004) werden würde. Am Beispiel des Begriffs ,Mulatte' zeigt Arndt, wie herabwürdigend dieser ist. Die Bezeichnung geht auf den portugiesischen Begriff ,mulo' zurück, was so viel bedeutet wie ,Maulesel' ${ }^{6}$. Menschen würden also mithilfe eines nicht fortpflanzungsfähigen Tieres zu beschrieben werden (Arndt 2004). Aber auch bei Bezeichnungen wie ,Naturvölker' oder ,Buschmänner' handelt es sich laut Arndt um Konstruktionen, die dazu dienen, afrikanische Menschen eng mit Natur und Tier zu verbinden, während „Europa als ,Kultur' konstruiert und so nicht nur zum überlegener[en] Gegenpol dargestellt, sondern als ,Norm“ gesetzt" (ebd.) wird. Im Gegensatz dazu steht Natur, die in diesem Zusammenhang, so Arndt, nicht positiv bewertet, sondern mit Attributen wie primitiv, ungebildet und von Instinkten geleitet, belegt wird. Auch der Blick auf ,Nordafrika' und 
,Schwarzafrika' deckt Arndt als eine zu Kolonialzeiten entstandene Aufteilung eines Kontinents in ein ,subsaharische[s] Afrika bar jeder Geschichte und Kultur" und „eine[m] weißen Norden, dem der Westen ein gewisses Maß an Kultur und Geschichte zubilligt" (Arndt 2004) auf.

Nachdem der Kolonialismus zusammenbrach wurde mithilfe der „Modernisierungstheorie"(Arndt 2005, S. 27)ein neues Model der Beherrschung erfunden. Hinter dieser der weißen, westlichen' Welt entsprungenen Theorie, steht die Annahme, dass die Welt keine andere Möglichkeit habe als „sich nach ihrem [dem ,westlichen', Anmerkung I.I.] Ebenbild zu organisieren und zu strukturieren" (ebd.). Diese zur Kolonialzeit konstruierte Vorstellung weißer Europäer_innen, deren Kultur und Religion angeblich höherwertiger sei als die Schwarzer afrikanischer Menschen, prägt das weiße und Schwarze Miteinander auch noch im 21. Jahrhundert (Reed-Andersson 2004). Dies zeige sich beispielsweise an Begrifflichkeiten, die noch bis heute zur Herabwürdigung von Schwarzen und ,People of Color' verwendet werden. Kilomba (2009) verdeutlicht aus psychologischer Perspektive, dass die Verwendung des Wortes ,Neger' ein koloniales Trauma reaktiviert, das Schwarze und ,People of Color' verwundbar bleiben lässt, wohingegen weiße sich durch diese Art der Beschimpfung ihre privilegierte Position sichern könnten (Kilomba 2009). Mit der Reduktion auf die Hautfarbe gingen abwertende Zuschreibungen wie „Animalität - Primitivität - Unwissenheit - Chaos Faulheit - Schmutz" (ebd.) einher. Auf diese Weise würde aus der biologisch determinierten Farbe der Haut automatisch Persönlichkeitsmerkmale abgeleitet, die Schwarze und ,People of Color' degradierten (ebd.). Auch in der in Kapitel zwei beschriebenen Migrationspolitik spiegeln sich die Machtverhältnisse wider, die den Umgang Europas mit Menschen aus afrikanischen Staaten noch bis heute kennzeichnen. Die repressive Politik an den Außengrenzen der EU verhindert gezielt die Einwanderung von Flüchtlingen aus Afrika. Die Migrationspolitik würde außerdem ,mit Bildern der Angst vor ,Überfremdung' [arbeiten, wodurch] eine modernisierte Form von Rassismus" (Kilomba 2009) geschürt würde, die sich negativ auf das Leben Schwarzer Deutscher in Deutschland auswirke. Fortgeführt wird dieser diskriminierende Umgang durch systematische polizeiliche Kontrollen Schwarzer Bürger_innen in Deutschland. Die unter der englischen Bezeichnung ,Racial Profiling' gefassten Kontrollen folgen Auswahlkriterien wie denen des ,physische[n] Erscheinungsbild[s], so genannte ,ethnische“ Merkmale oder [der] ,Rasse" einer Person" (Deutsches Institut für Menschenrechte 2013, S. 6) und werden ,als Entscheidungsgrundlage für polizeiliche Maßnahmen wie Personenkontrollen, Ermittlungen und Überwachungen" (ebd.) herangezogen. Auf Basis des Paragraphen 22 Abs. 1 BPolG, werden Menschen in Deutschland so allein aufgrund ihrer äußeren Erscheinung als vermeintlich Nicht-Deutsch eingeordnet und automatisch zu potentiell Kriminellen gemacht. Eine vom Deutschen Institut für Menschenrechte herausgegebe- 
ne Studie (2013) verdeutlicht, dass dieser Paragraph die Grund- und Menschenrechte verletzt, womit die deutsche Bundesregierung zur Abschaffung dieses Paragraphen aufgefordert ist - eine Forderung, die auch von Seiten der EU formuliert, in Deutschland jedoch bislang nicht umgesetzt wurde.

Postkoloniale Theorien und Kritische Weißseinsforschung zeigen, wie mithilfe von hegemonialen Diskursen aus der ,westlichen' weißen Perspektive heraus Bevölkerungsgruppen konstruiert und mit herabwürdigenden $\mathrm{Zu}-$ schreibungen belegt werden. Hinter Konstruktionen wie der vermeintlicher ,Rassen', stehen Macht- und Herrschaftsverhältnisse. Sie zielen damals wie heute darauf $a b$, die ,westliche' Vorherrschaft in der Welt auch in Zukunft sicherzustellen.

\subsection{Feministische Perspektive und die Konstruktion von Geschlecht}

Neben den bereits beschriebenen Theorien wird nachfolgend der Blick auf die Frauenbewegung und den feministischen Theorien liegen, da diese im Kontext weiblicher Genitalbeschneidung ebenfalls von Bedeutung sind.

Der Feminismus blickt auf eine lange Entstehungsgeschichte zurück und ist eng mit der Frauenbewegung verbunden. Er ist aber weder einer bestimmten Phase der Geschichte zuzuordnen, noch kulturell zu verorten (Lenz 2008, S. 860). Unter Frauenbewegung werden heute ,mobilisierende kollektive Akteur innen [verstanden], die sich in verschiedenen sozialhistorischen Milieus“ (ebd.) entwickelt haben und „einen grundlegenden Wandel der Geschlechterverhältnisse und damit verbundener gesellschaftlicher Ungleichheit und Abwertung" (ebd.) herbeigeführt haben.

Die Frauenbewegungen kann in zwei unterschiedliche Entwicklungswellen aufgeteilt werden, die „bürgerliche und proletarische Frauenbewegung im Kaiserreich (erste Welle) und [in die] Neue[ ] Frauenbewegung nach 1968“ (ebd., S. 860). Grundsätzlich ging es den Akteur_innen der Frauenbewegung um die „Emanzipation aus traditionellen Bindungen“ (Gerhard 1995, S. 262; in: Lenz 2008, S. 861) und der „Forderung nach gleichen Rechten“ (ebd.). Bereits in den 1950er Jahren entwickelte sich in Deutschland wieder ein traditionelles Verständnis entwickelte, welches Frauen stark in die „Privatheit der Familie“ (Lenz 2008, S. 861) drängte, während „Sphären der Öffentlichkeit, der Politik und des Rechts“" (ebd.) von Männern dominiert wurden. Diese Entwicklung brachte in den 1970er Jahren die Neue Frauenbewegung hervor, die sich für die Emanzipation aus diesen gesellschaftlich dominierenden Strukturen einsetzte. Es entwickelten sich zahlreiche Frauenorganisationen, die sich für Themen wie die politische und wirtschaftliche Teilhabe, aber auch für die sexuelle Selbstbestimmung der Frau (Schwangerschaftsverhü- 
tung, Verbot von Abtreibung, Strafbarkeit von Vergewaltigung in der Ehe usw.), (vgl. Lenz 2008, S. 861 ff.) engagierten.

Feministisch orientierte Organisationen finden sich auch in der pädagogischen Beratungsarbeit wieder. Spezifisch für die feministisch ausgerichtete Beratungsarbeit ist, dass sie sich von der damals gängigen individuellen Orientierung abwendet und sich stattdessen der Betrachtung ,geschlechtsspezifischer Lebensbedingungen und ihrer Auswirkungen auf psychische Strukturen und Handlungsmuster von Frauen“ (Pfingsten 1997, S. 85) widmet. Daraus haben sich unterschiedliche feministische Beratungskonzepte entwickelt, die wiederum von Grundsätzen wie Parteilichkeit und Betroffenheit geleitet werden (ebd.). Darüber hinaus zeichnet sich feministische Beratung dadurch aus, nicht allein den Rat suchenden Frauen zu helfen. Sie zielt zugleich darauf ab, die gesellschaftlichen, politischen und sozialen Strukturen zu verändern, die für die benachteiligte weibliche Position verantwortlich sind.

In der parallel zur Frauenbewegung entstandenen Frauenforschung, später dann Geschlechterforschung, wird der Fokus auf das „soziale Gewordensein von Frauen (und Männern)“ (Villa 2008, S. 146) gelegt. Geschlecht wird als eine gesellschaftlich konstruierte Kategorie gedacht und ist in ein biologisch determiniertes Geschlecht ,sex ' und ein gesellschaftlich konstruiertes Geschlecht, gender' unterteilt. Unter, gender ${ }^{6}$ werden die mit dem Geschlecht automatisch verbundenen Zuschreibungen gefasst, was sich konkret darin äußert, dass Männer beispielsweise mit Zuschreibungen wie Stärke, Aggression oder Ernährer belegt und Frauen als fürsorglich, empathisch und liebevoll konstruiert werden (vgl. Sauer 2006). Doch handelt es sich keineswegs um einen biologisch determinierten Automatismus, dass die Fähigkeit Kinder gebären zu können auch unweigerlich „Schwangerschaft und Gebären als schicksalhafte, natürliche Bestimmung der Frau“ angesehen werden muss, aus der sich „Mutterschaft und Fürsorge als natürliches Wesensmerkmal von Frauen ableite[n]" (Villa 2012, S. 75) lassen. Der Dualismus, der der Aufteilung der Gesellschaft in zwei Geschlechter zugrunde liegt, hat auch „eine soziale und kulturelle Differenz und Ungleichheit" (Villa 2012, S. 50) zwischen Mann und Frau mit sich gebracht, die sich in der Einteilung des öffentlichen Bereichs als „männlich-höherwertige Sphäre“ (ebd.) und der des familiären Bereichs als „weiblich-minderwertige Sphäre“ (ebd.) niederschlägt. Aus Villas Sicht handelt es sich bei der Konstruktion von Mann und Frau um „eine der hartnäckigsten Konstruktionen des Alltagswissen“ (ebd., S. 85).

Zur Analyse der Geschlechtskonstruktionen werden besonders von den Queer Studies Anfang der 1990er Jahre, die gesellschaftlichen Macht- und Herrschaftsstrukturen in den Blick genommen. Dem Machtbegriff Foucaults folgend werden ab diesem Zeitpunkt Geschlechtskonstruktionen und Sexualität unter Berücksichtigung dieser Strukturen untersucht. Vor dem Hintergrund von ,kulturellen Vorstellungen, sozialen Praxen und gesellschaftlichen Institutionalisierungsformen“" (Engel/Schuster 2007, S. 135) werden die bei- 
den Kategorien erforscht. Macht- und Herrschaftsverhältnisse konstruieren die Gesellschaft als zweigeschlechtliches und heterosexuelles System und schaffen anhand dieser Kriterien ein ,normal' beziehungsweise ,abweichend'. Gestützt durch unterschiedliche Fachdisziplinen wie Medizin, Psychiatrie, aber auch durch Medien, Wirtschaft, Politik und Religion wird eine Vorstellung von ,normaler' Sexualität und die Zugehörigkeit zu einem Geschlecht (mit den entsprechenden Zuschreibungen) konstruiert (vgl. ebd., S. 137). Es entstehen Schablonen, die am Ende dazu dienen, Sexualität und Geschlecht als ,normal' oder, unnormal ${ }^{`}$ zu diagnostizieren und durch verschiedene Therapieansätze an die gewünschte Norm anzupassen. Mithilfe von Normen und den damit verbundenen Normalisierungsprozessen wird versucht, aus einem heterogenen Feld, wie dem einer Gesellschaft, eine möglichst homogene Einheit zu schaffen. Normen sind aus der Sicht Foucaults gesellschaftlich ausgehandelte Regeln, die wie die Gesellschaft auch von Macht- und Herrschaftsverhältnissen geprägt sind (vgl. Engel/Schuster 2007). Mithilfe von Diskursen, worunter Foucault „mehr als die Gesamtheit von Aussagen zu einem bestimmten Thema“" (ebd., S. 136) fasst, sondern womit „Gesagtes ebenso wie Ungesagtes“ (ebd.) gemeint ist, werden Normen verhandelt. Wie wann was gesagt wird und was wiederum nicht gesagt werden darf, ist geregelt, verändert sich aber je nachdem zu welcher Epoche die Diskurse geführt werden. Am Beispiel von Geschlecht und Sexualität zeigt Foucault, dass diese beiden Kategorien ,nicht länger [...] naturgegebene, jenseits von Geschichte und Gesellschaft existierende Größen“" (Engel/Schuster 2007, S. 135) sind, sondern soziale Konstruktionen, die gesellschaftlichen und zeitgenössischen Veränderungen unterliegen. Der Blick auf die Geschichte verdeutlicht, wie sich der gesellschaftliche Umgang mit Sexualität wandelte. Während der letzten drei Jahrhunderte sei zu beobachten gewesen, dass sich zunächst etabliert habe, nicht mehr über Sexualität sprechen zu dürfen. Sie sei durch die „Sprachlosigkeit“" zensiert worden (Foucault 1991, S. 27). Diese Sprachlosigkeit sei ersetzt worden und heute einem Sprechen über Sexualität gewichen. Die „Diskurse über den Sex - spezifische, gleichzeitig nach Form und Gegenstand unterschiedliche Diskurse - haben unaufhörlich zugenommen" (ebd., S. 27) und seien laut Foucault maßgeblich von Kirche, Pädagogik, Medizin und Psychotherapie beeinflusst. Auf diese Weise werde bestimmt, mit welchem Vokabular gesprochen wird, mit welchen Personen es angebracht ist über Sexualität zu sprechen und am Ende auch was ,normale' Sexualität, was ,Perversion' ist (vgl. ebd.). Mithilfe dieser machtvollen Diskurse werden Kategorien wie Sexualität oder Geschlecht $\mathrm{zu}$ einem sozialen Konstrukt und etablieren sich in Form gesellschaftlicher Normen.

Auch Judith Butler nutzt in ihrer Analyse die Methoden Foucaults. Unter „diskurs- bzw. sprachtheoretischer Perspektive [...] [wird] die Fokussierung auf Sprache bzw. Diskurs als Ort und Modus der Konstruktion von Wirklich- 
keit sowie auf die Ausübung von Macht durch Diskursregime“ (Villa 2008, S. 148) verstanden. In Butlers Geschlechtertheorie ist „der ,Leib“ [...] selbst eine Konstruktion“ (Butler 1991, S. 26). Aus ihrer Sicht ist ,auch der Geschlechtskörper (,sex") ein [...] Effekt hegemonialer Diskurse" (Villa 2008, S.153). Der Körper wird aus dieser Perspektive zu einem ,passive[n] Medium, dem die kulturellen Bedeutungen eingeschrieben sind" (Butler 1991, S. 26), zwar sei genetisch festgelegt, welche Geschlechtsmerkmale sich entwickelten, doch würde die Einordnung in den Dualismus männlich - weiblich den menschlichen Körper prägen und bestimmte Eigenschaften kreieren. Butlers Theorie zielt darauf ab, ,zur Geschlechterverwirrung an[zu]stiften“ (Butler 1991, S. 61), um zu „kreativen Umgangsweisen mit dem Geschlecht“ (Villa 2008, S. 153) zu gelangen. Butler zeigt damit, dass der Körper Ausdruck gesellschaftlicher Normen ist, hervorgebracht durch Diskurse, die zeitgenössischen Wandlungen unterliegen, was somit auch für das Geschlecht gilt (Villa 2008, S.153). Durch die „Inszenierung einer angeblich natürlichen Substanz" (ebd.), durch die Geschlecht hervorgebracht wird, würden die sozialen Mechanismen verhüllt.

Am Beispiel des Umgangs mit Menschen, die mit nicht eindeutig zuweisbarem Geschlecht geboren werden, zeigen sich die Folgen der Geschlechterkonstruktion und der damit verbundenen Normalisierungsprozesse. Lange Zeit wurden die Genitalien von Kindern mit einer so genannten „Störungen der Geschlechtsentwicklung (Disorders of Sex Development, DSD)“ (Schweizer 2012, S. 26), auch Intersexuelle genannt, dieser Norm unterworfen und einem ,üblichen“ Geschlecht angepasst. Erst im Jahr 2008 wurde durch einen Bericht des Vereins Intersexuelle Menschen deutlich hervorgehoben, dass der bisherige Umgang mit Intersexuellen als ,,medizinische Versuche am Menschen" (CEDAW, S. 13 zit. in: Schweizer/Richter-Appelt 2012, S. 107) gelten und jeder operative Eingriff zur Genitalanpassung an Unmündigen als Menschenrechtsverletzung zu ächten sei. Doch führt dieser Bericht nur langsam zu einem Umdenken. Es existieren zwar Leitlinien im Umgang mit intersexuell geborenen Menschen, doch muss geprüft werden, ob die ,formulierten Vorschläge tatsächlich Eingang in die alltägliche medizinische Praxis finden“ (Schweizer/Richter-Appelt 2012, S. 111). In Deutschland ist die Eintragung in den Pass als, intersexuell' oder Angehörige eines ,dritten Geschlechts' trotz der Empfehlung des Ethikrates, nicht möglich.

Die dargestellte Entwicklung des Feminismus und sein Einfluss auf die pädagogische Beratung von Frauen und Mädchen zeigen, dass in diesem Kontext besonderes Augenmerk auf den gesellschaftlichen Macht- und Herrschaftsstrukturen liegt. Das Phänomen weiblicher Genitalbeschneidung kann aus dieser feministischen Perspektive zu dem Zeichen patriarchaler Herrschaft schlechthin werden, was bereits vor Jahrzehnten von manchen Feminist innen so formuliert wurde (Vgl. Braun/Levin/Schwarzbauer 1979). Fraglich bleibt jedoch, ob ein Modell, das in einem bestimmten gesellschaft- 
lichen Kontext entstanden ist und von Zugehörigen einer bestimmten Bildungsschicht vertreten wird(?), automatisch und ohne Berücksichtigung der historischen Entwicklung auf eine andere Gesellschaft übertragen werden kann. Spivak lehnt die Idee eines globalen Feminismus ab, da „es unmöglich ist, in einer universellen Geste alle Frauen repräsentieren zu wollen, ohne die Komplizenschaft mit imperialistischen Politiken zu analysieren" (Castro Varela/Dhawan 2005, S. 59). Der westliche Feminismus sei ein Diskurs, den privilegierte Akademiker_innen ins Leben gerufen hätten, der Blick auf die „'armen' Schwestern in der ,Dritten Welt"“" (ebd.) werde als Beispiel herangezogen und für die Durchsetzung feministischer Ideen missbraucht. Durch (post)koloniale Diskurse, in denen homogene Gruppen konstruiert und unter bestimmten Begriffen zusammengefasst werden, blieben laut Spivak all die unterschiedlichen Lebenserfahrungen der Individuen unberücksichtigt. Die Homogenisierung ganzer Bevölkerungsgruppen, so Spivak, bringe wiederum schwere politische Nachteile mit sich, insbesondere für diejenigen, die auf der untersten gesellschaftlichen Ebene stehen, da diese Menschen ,im Namen von ,Entwicklung' und ,Fortschritt' ausgebeutet werden“" (ebd., S. 56), ohne aber von der Entwicklung und dem Fortschritt profitieren zu können.

Eng mit der Einteilung der Gesellschaft in männlich - weiblich ist auch die Vorstellung von einem ,normalen' beziehungsweise , abweichendem' Genitale verbunden. Dabei handelt es sich um eine Vorstellung, die besonders über Medien verbreitet wird und den einzelnen Mitgliedern der Gesellschaft suggerieren, sich den erwünschten Bildern anpassen zu müssen - wenn nötig durch operative Eingriffe - mit dem Ziel nicht als ,abweichend" wahrgenommen zu werden. Kosmetische Chirurgie, darunter auch die kosmetische Genitalchirurgie, auch Intimmodifikation genannt, sorgt dafür, vermeintlich ,abweichende' Genitalien wieder ,normal' zu machen. Der Wunsch nach so genannten „Designervaginas“ (zit. n. Braun 2005 in: Borkenhagen 2010, S. 97), erfährt in den letzten Jahren stetigen Zuwachs (Filter/Reich 2012, S. 121). Mittlerweile hat sich die Technik der kosmetischen Chirurgie so weiterentwickelt, dass nahezu in jedem Bereich des weiblichen Genitales chirurgische Modifikationen durchführbar sind: „die Verengung der Vagina, die Verkleinerung der inneren Schamlippen, die Vergrößerung der äußeren Schamlippen, die Verkleinerung des Schamhügels, Reduktion der Klitorishaut, die Rekonstruktion des Jungfernhäutchen und das Aufspritzen der GPunkte-Region mit Kollagen“" (Borkenhagen 2010, S. 97). Obwohl bislang keine Studien über die möglichen Langzeitfolgen dieser Eingriffe bestehen, so scheint es sich um einen anwachsenden „kulturellen Trend“ (ebd., S. 98) zu handeln. Wo Frauen lange Zeit ausschließlich auf ihre Gebärmutter reduziert wurden, da sich die von der Kirche vertretene Meinung durchsetzte, dass „die weiblichen Geschlechtsorgane [...] ohnehin nur zur Fortpflanzung gut [sind]" (Sanyal 2009, S. 15), müssen sich Frauen der heutigen Zeit vor allem mit der äußerlichen Erscheinung ihres Genitales beschäftigen (vgl. Preiß 
2010). Wie schon in der Geschichte weiblicher Genitalbeschneidung aufgezeigt wurde, findet sich auch in diesem Zusammenhang das Argument wieder, unbeschnittene weibliche Genitalien seien unter gewissen Umständen beschnitten vorzuziehen. Paradoxerweise wird kosmetische Genitalchirurgie aber gleichzeitig betrieben, um die sexuelle Erregbarkeit der Frau zu steigern (Vgl. Dorndales de Andrade/ Jirovsky/Paloni 2010). Das heißt auf der einen Seite sollen Klitoris oder innere Schamlippen verkleinert werden, um Frauen ein Genitale zu formen, das nicht mit den Attributen ,triebhaft' oder ,sexuell ausschweifend' belegt ist. Gleichzeitig hat aber beispielsweise die Aufspritzung des G-Punktes das Ziel Frauen, mehr sexuelle Lust und Erregbarkeit zu geben. Obwohl auch diese Operationen keiner medizinischen Indikation folgen, sind sie erlaubt und sorgen innerhalb der deutschen Gesellschaft nicht für übermäßiges Entsetzen. Dies kann dazu führen, dass beschnittene Frauen aus Genitalbeschneidung praktizierenden Bevölkerungsgruppen mit Ablehnung auf diesen paradoxen gesellschaftlichen Umgang reagieren und sich möglicherweise in die Zeit des Kolonialismus zurück versetzt fühlen, in der sie (oder ihre Eltern) die Erfahrung gemacht haben, dass ihre Gesellschaft aus ,westlicher' Perspektive als minderwertig konstruiert wurde. Hinzu kommt, dass das ,westliche' Zurechtschneiden der Genitalien meist mit dem Argument begründet wird, es handle sich um erwachsene Frauen, die sich freiwillig diesem Eingriff aussetzen würden. Dieses Argument stößt bei etlichen afrikanischen Frauen auf Ablehnung, da somit „Frauen in FGM praktizierenden Gesellschaften Handlungsmacht und Entscheidungsfähigkeit über ihre Körper und die ihrer Töchter" (James/Robertson 2002 und Njambi 2004 in: Dorndales de Andrade/Jirovsky/Paloni 2010, S. 176) abgesprochen werde, „wohingegen der ,westlichen“ Frau autonome Entscheidungen über ihren Körper zugeschrieben werden" (ebd.). Ziel dieser Debatte ist nicht, die beiden Praktiken gegeneinander aufzuwiegen. Die Rückkopplungen an die deutsche Gesellschaft soll lediglich aufzeigen, dass sich unter europäischen wie auch afrikanischen Bevölkerungsgruppen Normvorstellungen des weiblichen Genitales etabliert haben, die bei Abweichungen zum Wunsch nach Anpassung führen kann.

\subsection{Intersektionalität}

Abschließend soll auf die intersektionale Perspektive eingegangen werden, da dies ein Ansatz ist, der nicht nur den theoretischen Hintergrund vervollständigt, sondern auch bei der Analyse der qualitativen Interviews mit beschnittenen Frauen bedeutsam ist. Die eng mit der Kritischen Weißseinsforschung verbundene Perspektive der Intersektionalität zeigt, dass soziale Kategorien nicht getrennt voneinander $\mathrm{zu}$ betrachten sind, sondern in ihren 
Überkreuzungen als Überlagerung von Macht- und Herrschaftsverhältnissen wahrgenommen und analysiert werden müssen. Das Konzept der Intersektionalität spiegelt die Situation betroffener Frauen wider, die von eben diesen vielseitigen ,Überkreuzungen' geprägt ist. Walgenbach definiert Intersektionalität folgendermaßen:

„Unter Intersektionaliät wird [...] verstanden, dass soziale Kategorien wie Gender, Ethnizität, Nation oder Klasse nicht isoliert voneinander konzeptualisiert werden können, sondern in ihrer ,Verwobenheit" oder ,Überkreuzung ${ }^{6}$ (intersections) analysiert werden müssen“" (Walgenbach 2012, S.81).

Demnach führt die Betrachtung einer einzelnen Kategorie zur Analyse sozialer Ungleichheit zu einem unvollständigen Bild, da die einzelnen Kategorien untereinander verbunden und miteinander verflochten sind. Mithilfe des intersektionalen Blickes wird aber nicht allein der Einfluss der verschiedenen sozialen Kategorien auf den Menschen analysiert, es geht vielmehr auch „um die Analyse ihrer Wechselwirkungen“" (ebd.).

Ursprünglich wurde die Debatte um Intersektionalität von Schwarzen Frauen eingeführt, da sie sich in der weißen, ,westlichen' feministischen Debatte nicht wieder finden konnten (Winker/Degele 2010, S. 11). Aus dem Blickwinkel dieser SchwarzenFrauen erfasste die Zugehörigkeit zur Kategorie Geschlecht alleine ihre Situation als Frauen nicht, da sie ebenso aufgrund ihrer Hautfarbe und Klassenzugehörigkeit Diskriminierungserfahrungen gemacht hatten. Die feministische Debatte wurde jedoch von weißen, der Mittel- oder Oberschicht angehörigen Frauen dominiert (Crenshaw 1989). Mithilfe eines Buchtitels aus der frühen Schwarzen Frauenbewegung fasst die Rechtswissenschaftlerin Crenshaw die Situation SchwarzerFrauen zusammen: „All the Women are White, All the Blacks are Men, But Some of Us are Brave" (Hull 1982 in: Crenshaw 1989, S. 139). Bislang seien die unterschiedlichen Kategorien, die zu ihrer Diskriminierung beitragen, nur getrennt voneinander wahrgenommen worden. Das Ausmaß der Diskriminierung SchwarzerFrauen könne, so Crenshaw, jedoch nur benannt werden, wenn man diese Bereiche miteinander verbinde (ebd.). Demnach würde es laut Crenshaw auch nicht ausreichen, SchwarzeFrauen in den Diskurs einzubeziehen, da die bloße Einbindung in feministische oder rassistische Diskurse ihrer Situation nicht gerecht werde (ebd., S. 140). Die Summe der intersektionalen Diskriminierungserfahrung sei größer als nur die Diskriminierung durch Geschlecht oder ,Rasse‘. Aus ihrer Sicht sei zur Erfassung der Situation SchwarzerFrauen der intersektionale Blick unumgänglich, da dieser die unterschiedlichen Diskriminierungserfahrungen miteinander verbinde und ihre Verwobenheit miteinander verdeutliche (vgl. Crenshaw 1989). Aus der Analyse von fünf Gerichtsurteilen, in denen „,die gleichzeitige Diskriminierung Schwarzer Frauen in Bezug auf Rasse wie auch Geschlecht wechselseitig ausgeblendet wurde" (Winker/Degele 2010, S. 12), entwickelte Crenshaw Anfang der 1990er Jahre ein Konzept der Intersektionalität. Es entstand die 
Vorstellung einer Straßenkreuzung, an der sich „Machtwege kreuzen, überlagern und überschneiden“ (Winker/Degele 2010, S.12).

In Deutschland ist Intersektionalität eng mit der Kritischer Weißseinsforschung verbunden, nicht zuletzt weil einige der daran beteiligten Forscher_innen beiden Debatten angehören (Lorey 2008). Ein Kritikpunkt an der intersektionalen Perspektive ist die Fokussierung auf Schwarze Frauen, da dies die Frage aufwirft, ob neben Klasse, ,Rasse' und ,Geschlecht' weitere Kategorien sozialer Ungleichheit unberücksichtigt bleiben (Winker/Degele 2010).

Winker und Degele sprechen von vier Kategorien, die soziale Ungleichheit kennzeichnen und die miteinander gedacht und verbunden werden müssen, um deren Tragweite erfassen zu können (vgl. Winker/Degele 2010). Eine Kategorie ist die der ,Klasse', die sich aus dem kapitalistischen System ableite, einem System, in dem der Zugang zu knappen Ressourcen ungleich verteilt ist, weil das System anders nicht funktionieren könne. Die unterschiedliche Klassenzugehörigkeit ergebe sich aus den „Kriterien Herkunft, Bildung und Beruf" (ebd., S. 42) und bestimme am Ende über den Zugang zum Arbeitsmarkt eines Menschen und der damit verbundenen gesellschaftlichen und finanziellen Position.

Die Kategorie ,Geschlecht', in Anlehnung an Butlerunterteilt in ein biologisch determiniertes Geschlecht ,sex' und ein konstruiertes ,gender', verbinden die Autorinnen Winker und Degele noch mit der Kategorie der sexuellen Orientierung (,desire') (Winker/Degele 2010, S. 45). Diese Kategorie und die damit verbundenen Zuschreibungen führten zu der Konstruktion einer zweigeschlechtlichen und heterosexuell geprägten Gesellschaft, die in einer naturalisierten, „d. h. unhinterfragten und selbstverständlich gemachten“ (ebd., S. 44) Weise zum Vorschein komme. Menschen, die dieser Konstruktion nicht entsprechen, würden als abweichend konstruiert und als Randgruppen marginalisiert werden.

,Rasse" als dritte Kategorie werde besonders in Deutschland aufgrund der Verwendung des Begriffes in nationalsozialistischen Zeiten durch Begriffe wie „Ethnie oder kulturelle Identität“ (ebd., S. 47) ersetzt, doch sehen die beiden Autorinnen dabei die Gefahr, dass durch diese Umbenennung Rassismus „verschleiert und salonfähig gemacht“ (ebd.) werde. Hinter der Konstruktion von ,Rasse“ stehe, ähnlich der Kategorie ,Geschlecht', ebenfalls ein eng an naturalistisch geprägte Vorstellungen gekoppeltes Bild der ,Anderen'. Das bedeute, dass diejenigen, die nicht Teil der Mehrheitsgesellschaft sind, „über eine andere Hautfarbe, Körperkonstitutionen, Ethnien, Religionen oder Weltanschauungen rassifiziert und damit zu Anderen gemacht" (ebd., S.47) werden. So werde aus dem Blickwinkel der Mehrheitsgesellschaft das ,Andere' konstruiert und dem , wir' untergeordnet. Dadurch, dass den konstruierten ,Anderen' in diesem Prozess eine untergeordneter Stellung zukomme, wovon die Zugehörigen der Mehrheitsgesellschaft profitierten, werde gleichzeitig 
das ,Andere" als minderwertig bewertet. Durch die Naturalisierung dieser Konstruktion werde Rassismus legitimiert.

Die vierte und letzte Kategorie bei Winker und Degele ist die des ,Körpers'. Der ,Körper' werde zunehmend als Produkt einer gesunden, aktiven Lebensweise konstruiert und der/die Einzelne dafür verantwortlich gemacht, wie funktionsfähig und gesund dieser ist, was gleichzeitig „Optimierungszwänge“ (ebd. S. 49) mit sich bringe. Für alternde oder behinderte Menschen werde die Kategorie des ,Körpers' zu einem Ausschlusskriterium, da sie deutlich von dieser ,wie auch immer konstruierten Norm“ (ebd., S. 50) abwichen. Aus dieser Konstruktion ergebe sich, dass sich nur (physisch und psychisch) gesunde Körper dafür eigneten, wirtschaftlich nutzbare Arbeitskräfte $\mathrm{zu}$ werden, während diejenigen, die aufgrund von Alter, Behinderung oder sonstigen körperlichen Merkmalen als abweichend konstruiert werden, von diesem Markt ausgeschlossen blieben. Da diese Konstruktion zusätzlich mit der Annahme verknüpft sei, dass jede/r Einzelne selbst für die Gesundheit und Leistungsfähigkeit des eigenen Körpers verantwortlich sei, werde dem/der Einzelnen suggeriert, selbst für den sozialen Ausschluss verantwortlich zu sein.

Da die verschiedenen Kategorien eng an gesellschaftliche Macht- und Herrschaftsverhältnisse gekoppelt sind, liegt bei der intersektionalen Perspektive der Fokus auf den Punkten, an denen sich die „Machtachsen“ (Lorey 2008) kreuzen und dafür sorgen, dass Menschengruppen Ausgrenzung oder Abwertung erfahren (Riegel 2012). Intersektionalität kann ,als Perspektive und Analyseblick verstanden" (ebd., S. 2) werden, mithilfe derer die auf unterschiedlichen sozialen Ebenen auftretenden gesellschaftlichen Machtund Herrschaftsverhältnisse analysiert und in ihrer Verwobenheit miteinander deutlich gemacht werden können (ebd.). Im Rahmen der Analyse von Forschungsdaten kann Intersektionalität genutzt werden, um, wie Riegel es formuliert, „Dominanzverhältnisse bzw. Differenzlinien“ (ebd., S.5) nicht schon vor der Durchführung der Forschung benennen zu müssen, sondern erst anhand des empirischen Materials zu rekonstruieren. Im Rahmen dieser Forschungsarbeit bietet sich daher die intersektionale Perspektive zur Analyse der qualitativen Interviews an.

\subsection{Exkurs: Zur Auseinandersetzung mit Kulturkonstruktionen}

Thematisch an die eben genannten postkolonialen Theorien und die Kritische Weißseinsforschung anschließend, wird nachfolgend auf die sozialwissenschaftliche Debatte um ,Kultur' eingegangen, die im Kontext beschnittener Migrantinnen in Europa von Bedeutung ist. Die Aussage, es handle sich 
bei dem Eingriff um einen Teil der ,Kultur' von Genitalbeschneidung praktizierenden Bevölkerungsgruppen, findet sich in der Literatur häufig wieder, weshalb nachfolgend in erster Linie der Frage nachgegangen wird, was ,Kultur ${ }^{6}$ bedeutet. Dies geschieht in Abgrenzung zum alltäglichen Sprachgebrauch, da der Begriff im Alltag zur Erklärung und Rechtfertigung von Verschiedenstem herangezogen wird und, je nach Kontext, negativ wie positiv konnotiert sein kann. Aufgrund der Vielschichtigkeit des Begriffes soll das Verständnis von ,Kultur' im Kontext dieser Arbeit eng gefasst werden.

Aus soziologischer Perspektive kann eine Definition wie beispielsweise Esser sie bietet bedeuten, dass unter ,Kultur' ,ganz allgemein - die erlernten oder sonst wie angeeigneten, über Nachahmung und Unterweisung tradierten, strukturierten und regelmäßigen, sozial verbreiteten und geteilten Gewohnheiten, Lebensweisen, Regeln, Symbolisierungen, Wert- und Wissensbestände der Akteure eines Kollektivs, einschließlich der Arten des Denkens, Empfindens und Handelns“ (Esser 2001, S. 1) verstanden werden. Auf Grundlage einer solchen Definition wird ,Kultur“ zu einem „Bezugsrahmen“ (ebd.), der den sozialen Akteuren einer Gesellschaft die Möglichkeit bietet, sich zu orientieren. Ohne diesen Rahmen der Orientierung wäre soziales Handeln zu komplex, dem/der Einzelnen wäre es nicht möglich ,alle jeweils existierenden Möglichkeiten und Regeln in einer Situation zu erkennen, ihre Folgen zu bedenken, zu bewerten und daraus eine sinnvolle Entscheidung zu treffen." (Esser 2001, S. 1).

Im Gegensatz dazu sehen Vertreter_innen der Cultural Studies ,Kultur nicht als „Gemeinsamkeit von Werten und Bedeutungen“ (Kalpaka 2005, S. 389) und verweisen auf das immer wieder zutage tretende „Fehlen eines Konsens in Wert- und Bedeutungsfragen“" (ebd.) innerhalb einer Gesellschaft. Vertreter innen der Cultural Studies sehen ,kulturelle Praktiken [...] eingebettet in historisch spezifische und sozial strukturierte Zusammenhänge" (Kalpaka 2005, S. 389). Definitionen von ,Kultur', besonders die daraus resultierende Zuordnung einzelner Menschen, ließen schnell den Charakter von etwas „Einheitliche[m] und Feste[m]“ (Leiprecht 2004, S. 8) entstehen und würden den Eindruck erwecken, alle Mitglieder einer ,Kultur' wüssten genau, woran sie sich zu orientieren haben, und würden ihr Verhalten gemäß geltender Regeln ausrichten. Zwar bietet ,Kultur' dem/der Einzelnen Orientierung und sorgt dafür, dass gesellschaftliches Handeln verstanden wird, doch darf nicht der Eindruck vermittelt werden, dass ,Kultur' sich auf eine staatliche Einheit beschränken lässt (vgl. Leiprecht 2004). Aus bestimmten ,kulturellen“ Eigenheiten, so Leiprecht, könnten sonst schnell ,nationale oder kulturelle Stereotype werden", woraus sich die Schwierigkeit ergebe, dass einzelne Individuen nicht mehr für sich stehen, sondern ,als Vertreter einer kulturellen Gruppe“" (Leiprecht 2004, S. 8) betrachtet würden. Definitionen von ,Kultur ' stünden daher einer alltäglichen Vorstellung von ,Kultur ${ }^{`} \mathrm{zu}$ nahe, eine Vorstellung, in der ,Kultur ${ }^{\varsigma}$ als homogene Gemeinschaft darge- 
stellt wird und einzelne Individuen dann allein durch die Zugehörigkeit zu dieser Gemeinschaft ,,bestimmte psycho-soziale Eigenschaften und Fähigkeiten aufweisen und in ihrem Denken, Fühlen und Handeln determiniert [sind]" (ebd., S.9). Angehörige der eigenen ,Kultur' würden aus dieser Perspektive heraus als individuelle und selbstbestimmte Personen beschrieben, denen es möglich sei, die Regeln ihrer ,Kultur' jederzeit zu hinterfragen und gegebenenfalls zu verändern. Angehörige ,anderer Kulturen' dagegen würden als Teil eines statischen Gebildes betrachtet und ,als Marionetten, die an den Fäden ihrer Kultur hängen, wahrgenommen“" (ebd.) werden. Ein in der Form unveränderlich gedachtes Verständnis von ,Kultur' kann, so Kalpaka, ebenso in ,rechte und rechtsextreme Diskurse eingebunden werden“ (Kalpaka 2004, S. 393), da ,Kultur' dann als natürliche Eigenschaft des Menschen konstruiert und ,als statisch und unveränderbar“ (ebd.) dargestellt wird. In diesen Diskursen wird eine so genannte ,kulturelle Identität' konstruiert, um Menschen anhand von äußeren Merkmalen ,in einer scheinbar neutral bestimmbaren Differenz zur ,deutschen Kultur““ (ebd.) zu beschreiben. Um solchen alltagsverständlichen oder ideologischen Diskursen von ,Kultur' zu entgehen, ist eine differenzierte Beschreibung des Begriffes nötig. Vertreter_innen wie Stuart Hall, einer der Mitbegründer der Cultural Studies, bieten eine solche Beschreibung. Die Cultural Studies widmen sich der „Analyse und Kritik kultureller Praxen“ (Kalpaka 2005, S. 389) und ,analysieren das Verhältnis von Macht und Widerstand" (ebd.), das sich hinter diesen verbergt. Aus Sicht der Cultural Studies umfasst

„die ,Kultur' einer Gruppe oder Klasse, die Lebensweise dieser Gruppe oder Klasse, die Bedeutungen, Werte und Ideen, wie sie in den Institutionen, in den gesellschaftlichen Beziehungen, in Glaubenssystemen, in Sitten und Bräuchen, im Gebrauch der Objekte und im materiellen Leben verkörpert sind. Kultur ist die besondere Gestalt, in der dieses Material und diese gesellschaftliche Organisation des Lebens Ausdruck findet. Eine Kultur enthält die ,Landkarte der Bedeutung', welche die Dinge für ihre Mitglieder verstehbar machen“ (zit. n. Clarke et al. 1979, S. 41; in: Kalpaka 2005, S. 390).

In dieser Beschreibung Clarkes sind mehrere besondere Wesensmerkmale von ,Kultur', zusammenzufassen. So werden zwar die Individuen „durch Gesellschaft, ,Kultur' und Geschichte geformt" (ebd.), doch ist zu betonen, dass ,jede Gruppe [...] irgendetwas aus ihren Ausgangsbedingungen macht" (ebd.). Als zentraler Aspekt ist somit das „Machen“ (ebd.) festzuhalten, das dem/der Einzelnen zwar nicht erlaubt ,Kultur' beliebig zu verändern, doch ist eine Entwicklung der ,Kultur „,im jeweils gegebenen geschichtlichen und gesellschaftlich bestimmten Feld der Möglichkeiten und Zwänge“ (ebd.,S. 390) möglich. Menschen sind an diesem Prozess beteiligt, auch wenn sie „,ihre Bedingungen [...] vorfinden“ (Kalpaka 2005, S. 391) sind sie dennoch als aktiv Handelnde ,und nicht als passive Empfänger_innen der Opfer der Verhältnisse“ (ebd.) zu betrachten. Leiprecht, dessen Kulturbegriff sich an 
dem Clarkes orientiert, verweist auf weitere wesentliche Begriffsmerkmale. Danach besteht ,Kultur ${ }^{6}$ aus einem „,bestimmten Repertoire von Bedeutungsmustern und Zeichensystemen (Werte, Normen, Bräuche wie andere Verhaltensregeln und, allgemeine Wissensbestände und ,Selbstverständlichkeiten', Traditionen, Rituale, Routinen, Glaubensvorstellungen, Mythen usw.)" (Leiprecht 2004, S. 11). All das würde, so Leiprecht, dazu beitragen, dass gesellschaftliches Miteinander verstanden werden könne, gleichzeitig würde es jede Gruppe oder Gesellschaft auch ,besonders" (Leiprecht 2004, S.11) machen, weshalb man "Kulturen“ auch als "besondere Lebensweisen“" (ebd.) kennzeichnen könne. Dies bedeutet aber nicht, dass innerhalb einer Gesellschaft oder Gruppe nur ein Bedeutungs- und Zeichensystem zu finden ist. Es existieren ,stets sehr verschiedene kulturelle Bedeutungsmuster/Zeichensystem und Lebensweisen" (Leiprecht 2004, S. 11), die von den Menschen selbst beeinflusst und verändert werden. Trotz der Unterschiede innerhalb einer Gesellschaft oder Gruppe, ist ein weiterer zentraler Gesichtspunkt kennzeichnend: Es gibt „dominierende Formen“ (Leiprecht 2004, S. 11), die über die Festlegung gewisser Normen bestimmen, anhand derer Verhalten als ,normal' beziehungsweise ,abweichend' gekennzeichnet werden, was ,mit Macht, Unterwerfung und Normalisierungszwang verbunden sein [kann]“ (Leiprecht 2004, S.11). Diese „Dominanz- und Machtverhältnisse“ (Kalpaka 2004, S. 391) sorgen dafür, dass „Kultur [...] ein von verschiedenen Gruppen umkämpftes Feld darstellt“ (ebd.), in dem Kategorien wie „Geschlecht, Klassenzugehörigkeit, Zugang zu Bildung, Migrationshintergrund, Alter, sexuelle Orientierung etc.“ (ebd.) zu Bezugspunkten bei der Bestimmung von Normalität oder Abweichung werden. In diesem Feld geht es für die einzelnen Menschen um den Kampf um Zugehörigkeit. Abschließend ist noch ein weiterer wichtiger Aspekt festzuhalten, der im Kontext dieser Arbeit von Bedeutung ist. Leiprecht verweist darauf, dass die Zugehörigkeit zu einer bestimmten ,kulturellen' Gruppe auch dadurch entsteht, sich von anderen ,kulturellen' Gruppen abzugrenzen. In diesem Abgrenzungsprozess wird die eigene ,kulturelle` Gruppe, trotz möglicher Differenzen, zu etwas Einheitlichem und Festen, das ,,alle Unterschiede innerhalb der Gruppe auszublenden“ (Leiprecht 2004, S. 11) versucht.

Auf Grundlage dieses Kulturbegriffs wird deutlich, dass es sich bei ,Kultur ${ }^{6}$ zwar um einen Kontext handelt, zu dem die einzelnen Menschen „in einem bestimmten Verhältnis“ (ebd., S. 12) stehen, der sie aber ,in ihrem Denken und Handeln nicht völlig" (ebd.) festlegt. Allein die Zugehörigkeit zu einem bestimmten ,kulturellen' Kontext sagt noch wenig über den einzelnen Menschen aus. Stattdessen ist ,das besondere Verhältnis der Individuen zu ihrer Geschichte, ihren Zugehörigkeiten zu möglicherweise verschiedenen kulturellen Gruppen, ihren kulturellen Hintergründen usw. ernst zu nehmen“ (ebd.,) und im Umgang mit den einzelnen Individuen zu berücksichtigen. 
Im Kontext weiblicher Genitalbeschneidung kann die immer wieder hervorgebrachte Frage, ob es ,westlichen' oder ,europäischen' Ländern zusteht, über ,Traditionen' ,nicht-westlicher' Bevölkerungsgruppen zu urteilen, nicht ignoriert werden. Hierzu werden nachfolgend unterschiedliche Perspektiven aufgezeigt, aus denen heraus dieser Diskurs betrachtet werden kann. Die Argumentationen sind auf unterschiedlichen Ebenen angesiedelt und so wird dieser Diskurs auch nicht von einer homogenen Einheit gebildet, sondern es gibt, wie Leiprecht es nennt, „dominante Formen“ (Leiprecht 2004, S. 11), die die von ihnen gesetzten Normen bestimmen wollen.

Aus der Perspektive der Cultural Studies ist dies eine Frage, die auf der „Vorstellung eines monolithischen Kulturverständnisses“ (Kalpaka 2005, S. 392) beruht. Das heißt, dass ,Kultur' als „ethnische oder nationale“ Zugehörigkeit verstanden wird, in der die einzelnen Mitglieder homogenisiert werden. Die ,westlichen' Länder verweisen darauf, dass es notwendig ist, die Menschenrechte, die aus, westlicher' Perspektive universelle Geltung haben, durchzusetzen. Befürworter_innen weiblicher Genitalbeschneidung in afrikanischen und asiatischen Ländern argumentieren hingegen, dass es sich bei den Menschenrechten um christlich und ,westlich` geprägt Werte handeln würde, die nicht auf jede Bevölkerungsgruppe der Welt zu übertragen seien (vgl. Mold/Reubi (Hg.) 2013). In diesem Zusammenhang ist der Verweis Bielefelds zu berücksichtigen, der die Nachzeichnung der Menschenrechtsentwicklung aus heutiger Sicht für schwierig hält. Die Geschichtsschreibung werde auf Pfeilern gestützt, die die Entstehung der Menschenrechte begünstigt haben, doch liefert diese Darstellung immer ein einseitiges Bild (vgl. Bielefeld 2007).

Aus der Perspektive der Kritischen Weißseinsforschung heraus betrachtet könnte das Festhalten an weiblicher Genitalbeschneidung als Folge der Kolonialzeit gewertet werden. In dieser Phase wurde Afrika und die dort lebenden Menschen zu einem ,homogenen und unterlegenen ,Anderen“ konstruiert, die der ,Zivilisierung' durch ,Europa' und der höher stehenden Europäer_innen geradezu bedurften (vgl. Arndt 2005). Dies ging mit der moralischen Verpflichtung der Europäer_innen einher, die ,unterlegenen Anderen“ zu zivilisieren (Arndt 2004). Eine Perspektive, die eine mögliche Erklärung dafür liefert, weshalb Menschen aus ehemals kolonialisierten Bevölkerungsgruppen sensibel auf, westliches' Einmischen reagieren.

Weiter zeigt die Erklärung beschnittener Frauen auf der Weltfrauenkonferenz von $1995^{15}$, in der diese die Welt um Mithilfe bei der Abschaffung weiblicher Genitalbeschneidung baten, dass aus der Position dieser betroffenen Frauen nicht automatisch rückgeschlossen werden darf, dass sie als Vertreterinnen aller Frauen aus Genitalbeschneidung praktizierenden Bevölkerungssche Gewalt in die Convention on the Elimination of all Forms of Discrimination of Women (CEDAW). 
gruppen gelten, da sich die Delegation zumeist aus gebildeten intellektuellen Frauen zusammensetzte, die im Gegensatz zu all den Frauen stehen, die diesen Zugang zu Bildung nicht haben und die der Diskurs um die Universalität der Menschenrechte nicht erreicht. In diesem Zusammenhang ist der Verweis auf Spivaks Beispiel der Witwenverbrennung (Castro Varela/Dhawan 2005, S. 56) wertvoll. Die Bitte der afrikanischen Frauen auf der Weltfrauenkonferenz in Peking um Unterstützung im Kampf gegen weiblichen Genitalbeschneidung ist demnach als ein Gesuch einer bestimmten Gruppe anzusehen, nicht als die Stimme, der Afrikanerinnen'.

Das Zusammenspiel der verschiedenen Komponenten führt dazu, dass eine Praktik, die auch mit negativen Konsequenzen verbunden sein kann, kein objektives Abwägen mehr erlaubt. Verteidiger innen weiblicher Genitalbeschneidung konstruieren den Eingriff als Teil ihrer ,Kultur' und lehnen ,westliche ${ }^{6}$ Einmischung ab, was für ,westliche` Aktivist_innen oder Organisationen zur Folge hat, sich mit diesem Vorwurf auseinandersetzen zu müssen. Vor dieser Debatte rückt dann der Wunsch der afrikanischen Aktivist_innen und selbst betroffenen Frauen nach Unterstützung in der Arbeit gegen die Beschneidung weiblicher Genitalien in den Hintergrund. Obwohl es die selbst betroffenen Aktivist_innen sind, die auf die Allgemeingültigkeit der Menschenrechte hinweisen und Genitalbeschneidung als Menschenrechtsverletzung einstufen, ist diese Gruppe der Aktivisti_innen nicht zwingend repräsentativ. Hier finden sich Parallelen zu anderen Menschenrechtsverletzungen oder Praktiken, die in der Wahrnehmung der, westlichen' Welt als ,grausam' benannt werden, die aber, wenn sie in ihrem Kontext betrachtet werden, gänzlich anders wahrgenommen werden können. Letztlich wird in dieser kontrovers diskutierten Frage keine abschließende, alle Menschen zufrieden stellende Antwort möglich sein. 


\section{Empirischer Teil}

\section{Methodik und Durchführung der Studien}

\subsection{Erkenntnisinteresse und Fragestellungen}

Im Rahmen der vorliegenden Forschungsarbeit wurden unterschiedliche Untersuchungen durchgeführt, die nachfolgend im Detail vorgestellt werden. Da es sich bei weiblicher Genitalbeschneidung um ein facettenreiches Phänomen handelt, zielten die Studien darauf ab, es aus verschiedenen Perspektiven zu beleuchten, was ein kompliziertes Forschungsdesign zur Folge hatte.

Das erste Erkenntnisinteresse lag darauf, herauszufinden, wie sich Präventions- und Beratungsangebote für bedrohte und beschnittene Mädchen und Frauen in Deutschland, England, Italien und der Schweiz momentan gestalten. Die in diesem Zusammenhang durchgeführte Untersuchung richtete sich an Fachpersonal verschiedener Einrichtungen und Kliniken, das in der Beratung und Begleitung beschnittener Frauen und deren Familien tätig ist. Im Zentrum dieser Untersuchung stand somit die Perspektive professionell Tätiger und zielte darauf ab, deren Blick auf weibliche Genitalbeschneidung zu analysieren und deren Umgang mit der genannten Zielgruppe zu untersuchen. Die Ergebnisse meiner Diplomarbeit (2006) haben zwar gezeigt, dass viele Einrichtungen bestehen, die ein großes Aufklärungs- und Beratungsangebot für bedrohte und betroffene Mädchen und Frauen anbieten, die Zielgruppe jedoch nur wenig damit erreicht wird. Aufgrund dieser Ergebnisse entstand die Frage, wie die Arbeit gegen weibliche Genitalbeschneidung künftig gestaltet werden kann, dass bedrohte Mädchen nachhaltig geschützt und betroffene Frauen mit unterstützenden Angeboten erreicht werden können. Daraus resultierte das der quantitativen Erhebung zugrunde liegende Interesse bezüglich der Herangehensweise weiterer Einrichtungen und Kliniken im Hinblick auf den Umgang mit von weiblicher Genitalbeschneidung bedrohten und betroffenen Mädchen und Frauen. Auf Basis dieser Frage wurde im Rahmen der Untersuchung ein teilstandardisierter Fragebogen entwickelt, der sich an Fachkräfte von Einrichtungen und Kliniken adressierte, die mit bedrohten und beschnittenen Mädchen und Frauen arbeiteten.

Die zweite Untersuchung richtete sich an betroffene Frauen und Männer, die aus einem Genitalbeschneidung praktizierenden Land in ein Genitalbe- 
schneidung ablehnendes Land immigrierten. Im Zentrum dieser Untersuchung standen die Perspektive der befragten Personen und die zentrale Frage, ob beziehungsweise inwiefern die Migration in ein weibliche Genitalbeschneidung ablehnendes Einwanderungsland die Sichtweise auf das Phänomen verändert. Die Daten für diese zweite Untersuchung wurden mithilfe qualitativer Methoden erhoben. Sie richtete sich an somalische Frauen und Männer, die im Laufe ihres Lebens nach Deutschland, England, Italien oder in die Schweiz immigriert sind. Zur Erfassung der Perspektive weiblicher Somalis wurde eine Kombination von biographisch-narrativen und problemzentrierten Interviews gewählt, die auf der Frage basierten, welche Handlungsstrategien beschnittene Frauen entwickelt haben, um mit den Veränderungen umzugehen, die mit dem Verlassen ihres Heimatlandes im Hinblick auf Genitalbeschneidung verbunden sind. Die Interviews mit den Frauen wurden in Anlehnung an die Grounded Theory ausgewertet. Die qualitativen Interviews mit den somalischen Männern wurden auf Basis problemzentrierter Interviews geführt und folgten der Frage, welche Sichtweise die männlichen Somalis auf weibliche Genitalbeschneidung haben. Die Interviews mit den männlichen Interviewpartnern wurden in den Hauptaussagen zusammengefasst und durch Interviewsequenzen gestützt.

Die Entscheidung für eine Kombination quantitativer mit qualitativen Erhebungsmethoden wird nachfolgend begründet. Dies erscheint nicht nur zum besseren Verständnis der vorliegenden Arbeit nötig, sondern auch, weil teilweise noch die Meinung vertreten wird, es handle sich um zwei Methoden, die aufgrund ihrer Entstehungsgeschichte und den dahinter stehenden Philosophien nicht miteinander kombiniert werden können (Kelle/Erzberger 2004, S. 299). Daran anschließend wird detailliert auf das Forschungsdesign der jeweiligen Untersuchungen eingegangen.

\subsection{Zur Kombination quantitativer und qualitativer Forschung}

Trotz vielseitiger Kritik zeigen einige Untersuchungen der letzten Jahre, dass eine Verbindung quantitativer Methoden mit qualitativen durchaus möglich und sinnvoll ist, da dies die Möglichkeit bietet, ganz ,unterschiedliche Aspekte desselben Phänomens“ (Kelle/Erzberger S. 303) zu beleuchten. Durch die Annäherung an den Forschungsgegenstand aus unterschiedlichen Perspektiven ist es eher möglich, der Komplexität eines Phänomens wie das weiblicher Genitalbeschneidung $\mathrm{zu}$ begegnen. Mithilfe der quantitativen 
Untersuchung wurde der Fokus auf die Perspektive der Fachkräfte gelegt, die gegen weibliche Genitalbeschneidung arbeiten. Neben den bestehenden Beratungs- und/oder Präventionsangeboten standen auch die Strukturen und Vernetzung der Einrichtungen mit anderen Fachbereichen im Vordergrund der Erhebung. Gleichzeitig zielte die Befragung darauf ab, den Fachkräften Raum für individuelle Sichtweisen zu geben, weshalb die Wahl auf einen teilstandardisierten Fragebogens gefallen ist, der an einigen Stellen Platz für offene Fragen ließ.

Qualitative Forschung dagegen bietet die Möglichkeit, „Lebenswelten ,von innen heraus', aus Sicht der handelnden Menschen zu beschreiben“" (Flick/Kardoff/Steinke 2004, S. 14), weshalb sich diese Forschungsrichtung zur Erfassung der Perspektive somalischer Frauen und Männer bezüglich weiblicher Genitalbeschneidung anbot. Ausgangspunkt für die Idee, die Perspektive von Somalis im Hinblick auf weibliche Genitalbeschneidung zu analysieren, war die Tatsache, dass bislang nur wenige wissenschaftliche Studien existieren, in denen die Sichtweisen Betroffener dargestellt werden, obwohl die Frage, was beschnittene Frauen an medizinischen, psychologischen oder pädagogischen Unterstützungsangeboten brauchen, nicht ohne die Zielgruppe erörtert werden kann. Hinzu kommt die mediale Aufbereitung des Themas, die Auswirkungen auf Betroffenen und deren Familien hat, da darin teilweise ein Bild transportiert wird, das oft wenig mit dem Bild von weiblicher Genitalbeschneidung zu tun hat, das betroffene Frauen haben. Die Frage, wie Betroffene die Diskussionen und die mediale Darstellung weiblicher Genitalbeschneidung im jeweiligen Einwanderungsland wahrnehmen, welche Emotionen diese in ihnen auslöst und welche Umgangsstrategien sie entwickelt haben, stand somit im Zentrum dieser Untersuchung. Da sich qualitative Forschung an den „Perspektiven der Beteiligten“ (Flick/Kardorff/Steinke 2004, S. 23) ausrichtet, war zur Erfassung der Sichtweise betroffener Frauen eine qualitative Forschungsmethode besonders geeignet, um „zu einem besseren Verständnis sozialer Wirklichkeit(en)“" (ebd.) beizutragen.

Die Kombination quantitativer mit qualitativen Forschungsmethoden zielt darauf $a b$, die unterschiedlichen Perspektiven und damit verbundenen Fragestellungen zu verdeutlichen. Ein Eingriff, der so kontrovers diskutiert wird wie der weiblicher Genitalbeschneidung, macht meines Erachtens eine Betrachtung aus vielen unterschiedlichen Perspektiven heraus erforderlich, um effektive Wege zu finden, Betroffenen und Bedrohten Angebote zu machen, die sie annehmen können. Am Ende der Auswertung der quantitativen Erhebung sowie der Analyse der qualitativen Interviews werden die zentralen Ergebnisse der Forschungsfelder und die theoretischen Erkenntnisse, die im vorangegangenen Kapitel benannt wurden, miteinander verbunden und diskutiert. 


\subsection{Methodisches Vorgehen bei der Befragung der Expert_innen}

Um ein möglichst breites Spektrum an pädagogischen Herangehensweisen und Beratungsstrukturen im Umgang mit Genitalbeschneidung zu untersuchen, wurde der Blick nicht allein auf die Arbeit gegen weibliche Genitalbeschneidung in Deutschland gerichtet, sondern ebenfalls auf Einrichtungen und Kliniken Englands, Italiens und der Schweiz. In den vier genannten Ländern existieren insgesamt 65 Einrichtungen und Kliniken, die gegen weibliche Genitalbeschneidung arbeiten, eine überschaubare Anzahl, die die Durchführung einer Totalerhebung ermöglichte. In Deutschland handelte es sich um 15 Beratungsstellen, 19 in England, 25 in der Schweiz und sechs in Italien. Die Kontaktdaten der Beratungsstellen wurden über einzelne Einrichtungen in den jeweiligen Einwanderungsländern ermittelt, da diese über Adresslisten aller im Lande tätigen Einrichtungen und Kliniken verfügen. Die überwiegende Mehrheit der Beratungsstellen sind eingetragene Vereine und Nichtregierungsorganisationen. In England existieren vor allem Kliniken, die sich im Rahmen ihrer Gesundheitsprogramme verstärkt gegen weibliche Genitalverstümmelung einsetzen. Zwar verfolgen alle Einrichtungen dasselbe Ziel, doch ist die Motivation und Herangehensweise teilweise sehr unterschiedlich. Manche Einrichtungen folgen feministischen Ansätzen, andere stützen ihre Aufklärungsarbeit vorwiegend auf die gesundheitlichen Folgen weiblicher Genitalverstümmelung, wieder andere Vereine setzen sich aus Betroffenen zusammen und arbeiten aufgrund persönlicher Erfahrungen gegen weibliche Genitalbeschneidung.

Nachfolgend werden die Vermutungen dargelegt, auf denen der teilstandardisierte Fragebogen basiert. Im Anschluss daran wird der Aufbau des teilstandardisierten Fragebogens erläutert.

Die zentrale Frage der quantitativen Erhebung galt dem professionellen Umgang mit von Genitalbeschneidung betroffenen und bedrohten Mädchen und Frauen von Seiten des in Einrichtungen und Kliniken tätigen Fachpersonals. Untersucht wurde in diesem Kontext, welche Wege gegangen werden, um Betroffene und Bedrohte mit konkreten Unterstützungs- und Präventionsangeboten zu versorgen und zu erreichen. Hierbei richtete sich die Untersuchung nicht alleine auf die betroffenen und bedrohten Mädchen und Frauen, sondern auch auf deren soziales Umfeld, da die soziale Gemeinschaft maßgeblich zum Erhalt der Praktik beiträgt. Dies spiegelt sich sowohl in den nachfolgend dargestellten Vermutungen wider als auch im Fragebogen selbst.

Die erste Vermutung war, dass Mitarbeiter_innen, die aus einer weibliche Genitalbeschneidung praktizierenden Bevölkerungsgruppe kommen, einfacher einen Zugang zu den betroffenen Frauen und Mädchen herzustellen können als Mitarbeiter_innen aus Bevölkerungsgruppen, die weibliche Geni- 
talbeschneidung nicht praktizieren. Diese Vermutung begründete sich auf den Ergebnissen meiner Diplomarbeit (2006). Diesen war zu entnehmen, dass es der Mehrheit der Beratungsstellen in Deutschland schwer fiel, Kontakt zu betroffenen und bedrohten Mädchen und Frauen herzustellen und sie mit ihren Angeboten $\mathrm{zu}$ erreichen. Ausnahmen bildeten die Migrant_innenvereine, die meist aus selbst betroffenen Mitarbeiter_innen bestanden und angaben, keine Schwierigkeiten beim Aufbau von Kontakt zu Betroffenen und deren Familien zu haben. (vgl. Ihring 2006 S.125ff.). Diejenigen Einrichtungen, die kaum Kontakt zur Zielgruppe hatten, begründeten dies damit, dass es sich um eine heikle Thematik handle, die nur in einem bestimmten Rahmen offen angesprochen werden könne. Außerdem gestalte es sich häufig schwierig, Kontakt zu Angehörigen von weiblicher Genitalbeschneidung praktizierenden Bevölkerungsgruppen aufzubauen (vgl. ebd.).

Die zweite Vermutung ergab sich aus der bereits in Kapitel eins dargestellten Situation, dass weibliche Genitalbeschneidung ein Phänomen ist, zu dessen Erhalt die gesamte Gesellschaft beiträgt, die Ergebnisse meiner Diplomarbeit aber gezeigt haben, dass Jungen und Männer in der Arbeit gegen Beschneidung nur selten beachtet werden (vgl. Ihring 2006), obwohl sie ebenfalls Teil der Gesellschaft sind und oftmals für den Erhalt weiblicher Genitalbeschneidung verantwortlich gemacht werden. Daraus ergab sich die Vermutung, dass Jungen und Männer in der Arbeit gegen Genitalbeschneidung noch immer unzureichend berücksichtigt werden. In diesem Kontext wurde nicht nur abgefragt, ob diese Zielgruppe mit Aufklärungs- und Beratungsangeboten versorgt wird, sondern auch, ob männliche Mitarbeiter in die Arbeit gegen weibliche Genitalbeschneidung eingebunden werden und die Angebote von entsprechender Zielgruppe angenommen werden.

Die dritte Vermutung bildete die Grundlage für das letzte Untersuchungsfeld und betraf die Vernetzung zwischen den Einrichtungen und anderen Fachkräften. Obwohl die Thematik bereits seit mehr als drei Jahrzehnten besonders bezüglich der daraus resultierenden medizinischen Konsequenzen erforscht wird, besteht die Annahme, dass noch immer zu wenig Gynäkolog_innen und Psycholog_innen existieren, die sich mit der Begleitung betroffener Mädchen und Frauen auskennen, was die Vermittlung von Beratungsstellen an informierte Gynäkolog_innen und Psycholog_innen erschwert. Diese Vermutung begründet sich auf den Untersuchungen Asefaws (2008) und Strenges (2013), die aufgezeigt haben, dass die Mehrzahl beschnittener Frauen negative Erfahrungen mit Gynäkolog_innen und Hebammen gemacht hat (Asefaw 2008, S. 74). Des Weiteren geht aus den Untersuchungen hervor, dass psychische Konsequenzen des Eingriffs durch die Migration verstärkt zutage treten, Betroffene aber häufig damit alleine gelassen werden (vgl. Asefaw 2008; Strenge 2013).

Auf Grundlage der genannten Vermutungen ist ein Fragebogen entstanden, der in fünf unterschiedliche Bereiche unterteilt war und sowohl aus 
standardisierten, geschlossenen als auch offenen Fragen bestand. Im ersten Bereich wurden allgemeine Informationen zur Einrichtung und der den Fragebogen beantwortenden Person erhoben. So wurde in diesem Teil abgefragt, ob die befragte Person ein_e hauptamtliche_r Mitarbeiter_in oder die Leitung der Einrichtung ist, welche Berufsausbildung diese hat, wie lange sie bereits an diesem Thema und in der Einrichtung arbeitet. Auch die Frage nach der eigenen oder der Herkunft der Eltern war Teil dieses ersten Abschnittes. Der zweite Bereich fragte nach dem Kontakt zu Betroffenen und Bedrohten und danach, wie sich dieser gestaltet. In diesem zweiten Teil wurde untersucht, welche Beratungsstellen Kontakt $\mathrm{zu}$ betroffenen Migrantinnen und deren Familien haben, wie viele Kontakte pro Jahr stattfanden, wie diese zustande kamen und ob dem Erstgespräch regelmäßige Beratungstermine folgten. Der dritte Bereich erfasste die Angebote der Einrichtung, die gemacht wurden, um bedrohte Mädchen vor der Praktik zu schützen. So stand in diesem Teil im Vordergrund, an wen sich die Angebote richteten und wie sie gestaltet wurden, wie regelmäßig sie stattfanden und ob sie von der Zielgruppe genutzt werden. Die persönliche Einschätzung der Mitarbeiter_innen bezüglich der Qualität ihrer Arbeit gegen Genitalverstümmelung war Teil des vierten Bereichs. In diesem Teil sollten die Befragten den Kontakt zu ihren Klientinnen einschätzen, bewerten ob ihre Angebote angenommen werden, den Kontakt zum sozialen Umfeld der Klientinnen beschreiben und einschätzen, ob sich ihre Arbeit mittlerweile unter den betroffenen Migrantinnen durchgesetzt hat. Im letzten Teil wurde schließlich nach der Vernetzung der Einrichtungen mit Gynäkolog_innen und Psycholog_innen gefragt. So wurde beispielsweise erhoben, ob ein regelmäßiger Austausch zwischen den genannten Berufsgruppen besteht, ob es möglich ist, Betroffene zu erfahrenen Gynäkolog_innen und Psycholog_innen zu schicken, und ob es Dinge gibt, die sich in der Vernetzung mit genannten Berufsgruppen verbessern könnten. Offene Fragen wurden besonders im Bereich der Präventionsarbeit und im Bereich der Fragen zu den konkreten Angeboten gestellt. Das dahinter stehende Ziel war, herauszufinden, welche Ideen hinter der Gestaltung der Angebote stecken und wie diese umgesetzt werden. Außerdem sollte mit den offenen Fragen vermieden werden, zu viele Vorgaben zu machen, in denen sich die den Bogen beantwortenden Personen nicht wieder finden können. Die Benennung der vermuteten Angebote in Form von Items hätte diesen Bereich eingeschränkt und nicht zu den erwünschten Ergebnissen geführt. Auch im letzten Bereich, der Vernetzung zu genannten Berufsgruppen, wurden einige offene Fragen gestellt, die Raum für Kritik und mögliche Verbesserungsvorschläge bezüglich der Vernetzung lassen sollten. Der komplette Fragebogen ist in der deutschsprachigen Version im Anhang der Arbeit einzusehen.

Der Fragebogen wurde zunächst in deutscher Sprache verfasst und erst in einem zweiten Schritt in die jeweiligen Landessprachen Englisch und Italienisch übersetzt. Da die Schweiz auch aus einem französisch sprechenden Teil 
besteht, wurde diesen Einrichtungen auf Anfrage die Möglichkeit gegeben einen französischen Fragebogen zu bekommen. Da bei der Übersetzung der Bögen besonders darauf zu achten war, die Fragen korrekt und sinngemäß zu übersetzen, hat die Übersetzung durch Muttersprachlerinnen stattgefunden, denen die Thematik weiblicher Genitalbeschneidung zudem nicht fremd ist. Da die beiden Übersetzerinnen auch Deutsch sprechen, konnten Unklarheiten in direkter Rücksprache geklärt werden. Die Übersetzung der offenen Fragen aus dem Italienischen wurde mithilfe einer in Deutschland lebenden italienisch sprechenden Übersetzerin gemacht. Die Versendung der quantitativen Fragebögen erfolgte per Email.

In Deutschland existieren 15 Beratungsstellen, zehn Einrichtungen haben den Fragebogen beantwortet. In der Schweiz sind es 25 Einrichtungen, wovon sieben den Fragebogen zurück geschickt haben. Von 26 englischen Einrichtungen haben ebenfalls sieben den Fragebogen beantwortet. In Italien wurden sechs Einrichtungen angeschrieben, drei haben sich an der Umfrage beteiligt. Die Auswertung der Befragung der Einrichtungen erfolgte in zwei Schritten. In einem ersten Schritt wurden die Antworten auf die geschlossenen Fragen ausgezählt. Diese wurde verschriftlicht und in einem Ergebnisteil zusammengefasst. Die Ergebnisse werden in Kapitel fünf dieser Arbeit pro Einwanderungsland präsentiert. In einem zweiten Schritt wurden die Antworten auf die offenen Fragen zusammengefasst. Diejenigen Antworten, die auf Italienisch waren, mussten hierfür ins Deutsche übersetzt werden. Anschließend wurden die zentralen Aussagen dieser offenen Befragung zusammengefasst und in die Präsentation der Ergebnisse eingefügt.

\subsection{Methodisches Vorgehen bei den qualitativen Interviews}

Zur Erfassung der Sichtweisen betroffener somalischer Frauen und somalischer Männer ist die Wahl auf das qualitative Interview gefallen. In den Interviews mit den Frauen wurden zwei Interviewmethoden miteinander kombiniert, das biographisch-narrative und das problemzentrierte Interview, während die Interviews mit den Männern ausschließlich problemzentriert ausgerichtet waren. Nachfolgend werden beide Interviewmethoden vorgestellt und erläutert, weshalb eine Kombination dieser beiden Methoden in den Interviews mit den Interviewpartnerinnen sinnvoll war.

Das biographisch-narrative Interview wurde von Fritz Schütze Mitte der 1970er Jahre entwickelt (Vgl. Schütze 1983). Im biographisch-narrativen Interview liegt das Interesse auf den „biographische[n] Deutungsmuster[n] und Interpretationen des Biographieträgers" (Schütze 1983, S.284), die, so Schütze, „nur im Zusammenhang seiner rekonstruierten Lebensgeschichte“ (ebd.) aufgedeckt werden können. Zur Einleitung des Interviews wird eine 
erzählgenerierende Frage gewählt, die die zu interviewende Person zur ,autobiographischen Anfangserzählung" (ebd., S.285) animieren soll und von der interviewenden Person nicht unterbrochen wird. Die mithilfe dieser Methode sichtbar werdenden subjektiven Bedeutungsstrukturen ermöglichen im Auswertprozess der Interviews die Rekonstruktion des subjektiven Sinnzusammenhangs. In Anlehnung an Fischer-Rosenthal und Rosenthal (1997) unterteilt sich das narrative Interview in unterschiedliche Phasen, in eben diese „Erzählaufforderung, die biographische Selbstpräsentation, das erzählgenerierende Nachfragen“ (Hopf 2000, S. 356). Die biographische Selbstpräsentation bildet den Hauptteil des Interviews und ist vor allem durch die Erzählung gekennzeichnet, erst im Anschluss daran wird konkreter nachgefragt. Grundsätzlich gilt, die Fragen sehr offen zu stellen, um den Erzählfluss weiterhin zu gewährleisten.

Die in den 1980er Jahren von Andreas Witzel entwickelte Forschungsmethode des problemzentrierten Interviews zielt ,auf eine möglichst unvoreingenommene Erfassung individueller Handlungen sowie subjektiver Wahrnehmungen und Verarbeitungsweisen gesellschaftlicher Realität" (Witzel 2000) ab. Während des Interviews ist auf eine sensible und akzeptierende Grundhaltung zu achten, da diese, so Witzel, das „Vertrauensverhältnis [...] [und] die Erinnerungsfähigkeit fördert und zur Selbstreflexion [motiviert]“ (Witzel 2000). Ziel ist es, den/die Interviewten ernst und wahrzunehmen, um einen Kontakt zwischen Interviewpartner_in und Interviewenden auf Augenhöhe herzustellen. Ein weiterer Vorteil des problemzentrierten Interviews ist, dass zwar das Individuum und dessen Sicht auf das jeweilige Problem im Fokus der Forschung stehen, die/der Forscher_in jedoch die eigenen Vorkenntnisse nicht verbergen muss. Stattdessen werden diese als Basis für den Interviewleitfaden genutzt, um während des Interviews einen Gesprächsrahmen zu schaffen, in dem das Problem benannt werden kann, gleichzeitig aber noch genug Raum für ein offenes und unvoreingenommenes Interview bleibt. So kann mithilfe des problemzentrierten Interviews die „dezidierte, spezifischere Fragestellungen [in den] Vordergrund“" (Mayring 1999, S. 52) gestellt werden, ohne die Offenheit zu verlieren. Die Interviewmethode ist stark an der Grounded Theory orientiert, die auch auf einem ständigen Vergleichen und Überprüfen das Datenmaterials beruht, da Theorie während des Forschungsprozess entsteht und grundsätzlich modifizierbar ist (vgl. Witzel 2000).

Des Weiteren orientiert sich das problemzentrierte Interview an einem vorab geplanten Interviewleitfaden, der sich in den Interviews mit betroffenen Frauen nur in wenigen Fragen von denen mit den Männern unterschied. Beide Interviewleifäden richteten sich an nachfolgenden Regeln des problemzentrierten Interviews. Zur Vorbereitung des Interviewleitfadens wird in einem ersten Schritt das Problem analysiert, das Gegenstand der Interviews sein soll, woraus sich dann in einem zweiten Schritt die Hauptaspekte für die 
Interviewbefragung ableiten lassen, die von der/dem Forscher_in im Leitfaden festgehalten werden (Witzel 2000). Ziel eines Leitfadens ist dem/der Forscher_in während der Interviews einen gewissen Rahmen zu geben, indem festgehalten ist in welcher Reihenfolge bestimmte Aspekte des Problems angesprochen werden. Hauptaugenmerk liegt dabei auf der Einstiegsfrage, da diese dazu dient gleich zu Beginn eine Atmosphäre zu schaffen, die dem/der Interviewten ermöglichen soll, offen über die angesprochene Thematik zu reden. Diese Einstiegsfrage enthält bereits das zu thematisierende Problem, lässt dem/der Interviewten gleichzeitig jedoch noch viel Freiraum für offene Erzählungen (Witzel 2000). In Anlehnung an Witzel sollte der Leitfaden des Weiteren aus verschiedenen Fragekategorien bestehen, die neben den ,erzählungsgenerierenden" Fragen (Witzel 2000, S. 5) auch „Sondierungsfragen“ (Mayring 1999, S. 52) enthalten, die dazu dienen herauszufinden, „ob das Thema für den einzelnen überhaupt wichtig ist bzw. welche subjektive Bedeutung es für ihn besitzt" (ebd.). Des Weiteren kann dieser Teil zu einem „'Hervorlocken“ konkreter Erfahrungsbeispiele oder biographischer Episoden“ (Witzel 2000, S. 5) anregen sowie ,abstrakte, fehlende oder unklare Begriffe [verdeutlichen] und [...] konkrete Bezüge zu Kontextbedingungen des Handelns her[stellen]“ (ebd.). Die „verständnisgenerierenden Fragen“ (ebd.) regen die Gesprächspartner_innen zur Selbstreflexion an und sorgen dafür, dass die eventuell von dem/der Forscher_in vermittelte Meinung entschärft wird, so dass die Meinung der interviewten Person zum Ausdruck kommen kann und möglichst wenig durch die des Forschenden gefärbt ist. Aber auch Verständnisfragen gehören zu diesem Teil der Interviewführung, die darauf abzielen Widersprüchlichkeiten zu klären oder „Alltagsselbstverständlichkeiten der Interviewten" (ebd.) aufzubrechen. $\mathrm{Zu}$ dieser Fragekategorie gehören auch die „Ad-hoc-Fragen“ (ebd.), die dann gestellt werden, wenn während des Interviews Aspekte auftreten, die der/die Forscher_in bei der Formulierung des Leitfadens nicht bedacht hat, bei denen sich aber während des Interviews herausstellt, dass es sich um bedeutsame Themen handelt oder dass sie „für die Erhaltung des Gesprächsfadens bedeutsam“ (ebd.) sind. Wann von den erzählungsgenerierenden Fragen zu den verständisgenerierenden Fragen gewechselt wird, ergibt sich im Interviewprozess. Laut Witzel ist es sinnvoll, mit den erzählungsgenerierenden Fragen zu beginnen, da diese das Vorwissen des Forschenden mit den subjektiven Erzählungen der Befragten kombiniert und dem/der Forscher_in ermöglicht, Muster aus Gesagtem zu verstehen. Mithilfe verständnisgenerierender Fragen wiederum wird versucht, neue Muster herauszufinden, die aus der Sicht des/der Interviewten selbst hervorgehen, ohne deren Sichtweise durch das Vorwissen der Interviewenden zu beeinflussen. 


\subsection{Forschungszugang und Sample der qualitativen Untersuchung}

Mit dem Wissen, dass es sich bei weiblicher Genitalbeschneidung um ein Thema handelt, das unter Betroffenen und deren Familien nicht häufig thematisiert wird, stellte sich anfangs die Frage, wie Somalis gefunden werden konnten, die bereit wären, mit einer ihnen fremden Person über diese intime Thematik zu sprechen. Die Annahme, dass es nicht leicht werden würde, somalische Frauen und Männer zu finden, führte dazu, dass Personen, die bereits im Kontakt mit Somalis waren, genutzt wurden, um das Anliegen und Ziel der Interviews zu erklären und abzuklären wer zu einem Interview bereit wäre. Vor diesem Hintergrund war besonders wichtig, dass potentiellen Gesprächspartner_innen schon im ersten Kontakt verdeutlicht wurde, dass es sich bei den Interviews um einen Gesprächsrahmen handeln würde, der von großer Offenheit und Akzeptanz geprägt ist und die Interviews nicht darauf abzielten, die Interviewpersonen von einer bestimmten Meinung zu überzeugen. Dennoch bestand die Vermutung, dass sich eher Gesprächspartner_innen finden lassen würden, die selbst Gegner_innen der Praktik sind. Die Auswahl der Interviewpartner_innen konnte aus diesen Gründen nicht bestimmten Kriterien folgen, da diejenigen Somalis interviewt wurden, die zu einem Interview bereit waren. Das Sample wurde erst bei der Analyse der Interviews festgelegt.

Die Suche nach Interviewpartner_innen verlief von Untersuchungsland zu Untersuchungsland unterschiedlich. In Deutschland und der Schweiz wurde telefonisch Kontakt zu somalischen Kultur- und Migrant_innenvereinen hergestellt. In den Telefontaten wurde das Anliegen erklärt, verbunden mit der Frage, ob sich aus ihrem Verein jemand finden lasse, der/die zu einem Interview bereit sei. Nachdem sich anfangs niemand finden ließ, wurde die Strategie verändert und mehr auf mein persönliches Interesse an der Meinung der Interviewpartner innen eingegangen. Ich erklärte, dass ich aufgrund meines eigenen somalischen Hintergrunds, ein persönliches Interesse an der Meinung der Somalis zu diesem Thema hätte und mich daher freuen würde, wenn sich Somalis finden ließen, die sich zu einem Interview bereit erklärten. In nahezu allen Fällen waren die Personen daraufhin bereit zu einem Interview, begründeten ihre Entscheidung auch häufig mit dem Argument, dass sie nicht mit Deutschen über dieses Thema reden würden, sie aber zu einem Interview bereit seien, da ich ja ein Stück weit ,eine von ihnen“ sei. Zum anderen konnten weitere Interviewpartner_innen über eine somalische Freundin gefunden werden, die den Kontakt zu den entsprechenden Personen herstellte. So wurden in Deutschland insgesamt vier Personen interviewt, zwei somalischen Frauen und deren Ehemänner. In der Schweiz erklärten sich drei Personen zu einem Interview bereit, zwei somalische Frauen und ein Mann. 
In England verlief der Kontakt zu Interviewpartner_innen über die Hebamme einer Klinik, die besonders viele somalische Patientinnen betreute. Dieselbe Hebamme hatte an der Befragung der Beratungsstellen teilgenommen, woraus ersichtlich wurde, dass ihr Arbeitsschwerpunkt auf der medizinischen Begleitung somalischer Frauen (und deren Familien) lag. Mit dieser Hebamme wurde per Email Kontakt aufgenommen und gefragt, ob es ihr möglich wäre, Interviewpartner innen zu organisieren, die über diese Thematik sprechen wollten. Sie vermittelte Kontakte zu zwei somalischen Frauen und einem somalischen Mann.

Italien war das einzige Untersuchungsland, in dem die Kontaktaufnahme und die Interviews mithilfe einer Dolmetscherin erfolgten. Die beiden männlichen Interviewpartner konnten über die Schwester der Dolmetscherin zu einem Interview gewonnen werden, die beiden weiblichen Interviewpersonen wurden von der Dolmetscherin auf der Straße angesprochen und gefragt, ob sie zu einem Interview zum Thema Genitalbeschneidung bereit wären. Da sehr viele Somalis in Italien leben, war es nicht schwierig Personen zu finden und anzusprechen. Ohne Dolmetscherin wäre dies jedoch nicht möglich gewesen, da sie sowohl Somali als auch Italienisch sprechen und sich ganz auf die Sprachmöglichkeiten der Menschen einlassen konnte. Dank ihr war es möglich, insgesamt vier Interviews mit zwei somalischen Frauen und zwei Männern zu führen.

Die Interviewsettings gestalteten sich ebenfalls unterschiedlich. In Deutschland wurde ich zu beiden Ehepartnern nach Hause eingeladen. Dort ging es familiär zu, da in beiden Familien auch deren Kinder zugegen waren, die an manchen Stellen für Unterbrechung gesorgt haben. Trotzdem war es möglich, das Interview mit den jeweiligen Interviewpartner_innen weitgehend in Ruhe zu führen, da wir uns dazu in einen anderen Raum zurückziehen konnten.

In England wurden zwei der drei Interviews in den Räumlichkeiten der Klinik, in der die beiden Interviewpartner_innen tätig waren, durchgeführt. Das dritte Interview dagegen fand in einem Restaurant statt und musste daher immer wieder unterbrochen werden.

In der Schweiz fanden die beiden Interviews mit den Frauen ebenfalls bei ihnen zuhause statt. Diese waren von sehr viel Ruhe geprägt, da wir nur zu zweit waren und das Interview zu keinem Zeitpunkt unterbrochen werden musste. Insgesamt waren die Interviews in der vertrauten heimischen Umgebung von großer Offenheit und Intimität geprägt, die Gesprächspartner_innen machten während der Gespräche nicht den Eindruck, beschämt oder gehemmt zu sein. Das Interview mit dem somalischen Mann in der Schweiz fand dagegen in einem Café statt, sowie auch alle Interviews in Italien in Cafés oder Restaurants geführt wurden. Diese Situationen waren deutlich unruhiger, da noch weitere Personen um uns herum waren, die sich unterhielten, und an vielen Stellen noch einmal nachgefragt werden musste. Oft ent- 
stand der Eindruck, dass besonders die Frauen in Italien gehemmt waren, in der Öffentlichkeit über dieses intime Thema zu sprechen, da sie an etlichen Stellen leiser oder undeutlicher sprachen. Bei den Interviews in Italien war die Situation durch die notwendige Übersetzung der Interviewfragen durch die Dolmetscherin insgesamt unruhig. Es wurde immer wieder zwischen Somali, Italienisch und Deutsch gewechselt, was sowohl die Interviewsituation vor Ort als auch die Transkription der Interviews deutlich erschwerte. Die Dolmetscherin sorgte durch kurze zusammenfassende Übersetzungen des Gesagten dafür, dass ich als Interviewende den Kontext verstand, um weitere Fragen stellen zu können. Dennoch wurde nach der Transkription, die mithilfe der Dolmetscherin für die somalischen Passagen und mithilfe einer weiteren Übersetzerin für die italienischen Anteile erfolgte, festgestellt, dass manche Fragen nicht treffend formuliert waren oder an anderen Stellen hätten gestellt werden müssen. Trotz der teilweise widrigen Interviewsituationen, besonders in Italien, äußerten sich die Interviewpartner_innen ohne Umschweife zur Thematik.

Am Ende der Interviewphase sind insgesamt 14 Interviews geführt worden, davon acht mit somalischen Frauen und sechs mit somalischen Männern. Da die Interviewpartner_innen nicht nach bestimmten Kriterien gesucht werden konnten, erfolgte die Wahl der auszuwertenden Interviews erst nach der Transkription und der wiederholten Durchsicht des Materials. Nachdem die Interviews geführt wurden, wurden alle transkribiert. Zur Transkription und Auswertung wurde das Computerprogramm Atlas.ti verwendet, ein spezielles Analyseprogramm zur Auswertung qualitativer Interviews.

\subsection{Reflexion des Interviewverlaufs}

Die Interviews mit den Frauen zielten darauf ab, zu untersuchen, welche Handlungsstrategien sie im Hinblick auf weibliche Genitalbeschneidung in einem Einwanderungsland, das die Praktik ablehnt, entwickelt haben. Im Hinblick auf somalische Frauen und deren Geschichte mit weiblicher Genitalbeschneidung darf der Sozialisationsprozess nicht außer Acht gelassen werden, da die Frauen die mit dem Eingriff verbundene Bedeutung durch diesen Prozess erlernen. Die Bedeutung, die der Eingriff für genital beschnittene Frauen hat, unterscheidet sich vermutlich von den Vorstellungen, die Frauen mit dem Eingriff verbinden, die einer Bevölkerungsgruppe angehören, die den Eingriff nicht praktizieren. Mithilfe des biographisch-narrativen Interviews sollte den weiblichen Interviewpartnerinnen die Möglichkeit gegeben werden, über ihre Kindheit und Jugend in Somalia zu sprechen, ohne dabei aber direkt Genitalbeschneidung in den Mittelpunkt zu stellen. Gleichzeitig aber wurde auf diese Weise der Lebensabschnitt der Frauen beleuchtet, 
in dem das Phänomen weiblicher Beschneidung für sie seine Bedeutung bekommen hat und in dem sie selbst die Praktik erlebt haben. Die Einstiegsfrage im biographisch-narrativen Interview lautete: „Erzähl doch mal, wie war dein Leben bevor du Somalia verlassen hast? " und zielte darauf ab, die Frauen dazu zu ermuntern, von ihrem Leben in Somalia zu berichten, ohne jedoch festzulegen mit welchem Lebensabschnitt begonnen werden sollte. Lieferte diese Einstiegsfrage nicht den nötigen Erzählimpuls, wurden vorab noch weitere Fragen formuliert, die sehr viel konkreter waren und darauf abzielten, den Interviewpartnerinnen den Einstieg zu ermöglichen. Diese Fragen bezogen sich dann auf bestimmte Lebensabschnitte ihres Lebens in Somalia, wie beispielsweise konkrete Fragen zu ihrer Kindheit, dem Besuch der Schule oder ihrer familiären Situation in Somalia. Obwohl bestimmte Fragen vorab überlegt wurden, sollte dieser Teil des Interviews weiterhin den Charakter einer freien Erzählung haben, in der sich die Interviewpartnerinnen nicht zu bestimmten Erzählungen gedrängt fühlen sollten. Sie sollten entscheiden, was sie von ihrem damaligen Leben erzählen wollten und ob sie in diesen Erzählungen bereits auf genitale Beschneidung zu sprechen kommen wollten. Diese Vorüberlegung war von Bedeutung, da die Entscheidung, über Genitalbeschneidung im Kontext der eigenen Biographie zu reden, für die Interviewpartnerin gleichsam bedeuten kann, über die eigene Beschneidung sprechen zu müssen. Ob beziehungsweise wie ausführlich sie ihre eigene Beschneidungsgeschichte thematisieren, sollte ihnen überlassen bleiben, nicht nur weil es sich um ein sehr intimes Thema handelt, sondern auch weil eine Retraumatisierung durch bestimmte Fragen vermieden werden sollte. Im Verlauf der Interviews mit den Interviewpartnerinnen stellte sich jedoch heraus, dass die Interviewpartnerinnen selbst meist sehr schnell auf die eigene Beschneidung zu sprechen kamen. Ohne Umschweife oder Scham wurde das Thema aufgegriffen, oft noch bevor sie überhaupt viel zu ihrem Leben in Somalia erzählt hatten. Somit muss im Rückblick festgehalten werden, dass die geplante Trennung der unterschiedlichen Interviewphasen nicht grundsätzlich eingehalten werden konnte.

Des Weiteren haben manche Interviewpartnerinnen den biographischnarrativen Teil des Interviews genutzt, um ausgiebig über ihre Kindheit und Jugend in Somalia zu berichten, andere wiederum sparten diesen Teil der Erzählung nahezu aus, antworteten nur auf die diesbezüglich gestellte Fragen und wechselten dann wieder zu dem Gesichtspunkt, der aus ihrer Sicht von Bedeutung war. Die Entscheidung, den biographisch-narrativen Teil in den Interviews mit den männlichen Somalis wegzulassen, wurde getroffen, da die Interviews mit den Männern darauf abzielten, deren Meinung zum Thema weibliche Genitalbeschneidung zu hören und sich hierfür die Methode des problemzentrierten Interviews eignete. Meines Erachtens spielt die Biographie der Männer in Bezug auf weibliche Genitalbeschneidung zwar auch eine Rolle, da sie ebenso Teil einer Genitalbeschneidung praktizierenden Bevölke- 
rungsgruppe darstellen, doch verkörpern sie den Eingriff nicht in der Form wie dies betroffene Frauen tun.

Der Verlauf des problemzentrierten Interviews unterschied sich zwischen den interviewten Frauen und Männern nur in wenigen Fragen. Sonst folgte er einem festgelegten Ablauf, der bereits mit einem Kurzfragebogen zur Erfassung persönlicher Daten (wie Alter, Beruf, Wohnort) der Interviewpartner_innen als Einstieg begann. Die eigentliche Interviewsituation konnte so von dieser Frage-Antwort-Situation befreit werden und die persönliche Sicht der Befragten zum Thema Genitalbeschneidung in den Vordergrund stellen (vgl. Witzel 2000). Außerdem konnten diese Daten in den Interviews mit den männlichen Personen dazu genutzt werden, um den Gesprächseinstieg zu erleichtern, da daraus bereits Informationen ersichtlich wurden, mit denen das Interview begonnen werden konnte (vgl. SchmidtGrunert 1999).

Die dem Kurzfragebogen folgende, in die jeweilige Landessprache übersetzte Einverständniserklärung stellte sicher, dass die Interviewten darüber aufgeklärt wurden und damit einverstanden waren, dass die Interviews aufgezeichnet, transkribiert und in anonymisierter Form zu Forschungszwecken genutzt werden. Im problemzentrierten Interviewteil wurde konkret über weibliche Genitalbeschneidung gesprochen. Auch in diesem Teil war von besonderem Interesse, ob beziehungsweise inwieweit sich die Sichtweise der Interviewpartner innen durch ihre Migration in eine weibliche Genitalbeschneidung ablehnende Gesellschaft verändert hat. In den Interviews mit den somalischen Männern war außerdem besonders interessant, wie sie zu dem Argument stehen, weibliche Genitalbeschneidung werde gemacht, weil Männer an dem Eingriff festhalten würden. Die Interviews mit den somalischen Männern verliefen meist nach dem Interviewleitfaden, nur in Italien war es schwer sich daran zu orientieren, da die Situation durch die Notwendigkeit einer Dolmetscherin insgesamt unruhig und teilweise durcheinander war. Insgesamt ist jedoch festzuhalten, dass sowohl die männlichen als auch weiblichen Interviewpartner_innen von großer Offenheit waren und ohne Umschweife von weiblicher Genitalbeschneidung und den Konsequenzen daraus sprachen.

\subsection{Auswahl der Interviews}

Von acht qualitativen Interviews mit den somalischen Frauen wurden am Ende fünf Interviews analysiert und nachfolgend in Form von Fallanalysen dargestellt. Es wurde entschieden pro Einwanderungsland ein Interview zu analysieren, einzige Ausnahme bilden die beiden Interviews in Italien. Diese Auswahl wird nachfolgend begründet. 
Saras Interview wurde für Deutschland ausgewählt, weil es von großer wiederkehrender Ambivalenz geprägt ist. Die Frage, wer an ihrer Beschneidung Schuld hat, zieht sich durch das gesamte Interview, besonders interessant daran ist allerdings, dass Sara sich diese Frage erst stellt nachdem sie Somalia verlassen hat und in Deutschland erfahren hat, dass Genitalbeschneidung keine Vorteile hat. Vorher war sie von dem Eingriff überzeugt, heute bereut sie es in einem Land aufgewachsen zu sein, in dem weibliche Genitalbeschneidung als positiver Schritt im Leben eines Mädchens präsentiert wird. Die Analyse des Interviews mit Sara ist deutlich länger als die der restlichen Interviewpartnerinnen, was daran liegt, dass Sara viel von ihrer Kindheit und Jugend in Somalia berichtet und diese Erzählungen für den Verlauf der Interviewanalyse bedeutend sind, da sie den Bruch, den die Migration nach Deutschland in ihrem Leben darstellt, und die damit verbundene Zerrissenheit beschreiben. Sara ist die einzige Interviewpartnerin, bei der ihre 15 Jährige Tochter während des Interviews als Zuhörerin dabei saß.

Nadifa aus der Schweiz kündigte bereits im ersten Telefonkontakt an, dass sie gerne das Interview führen wolle, da sie dieses nutzen möchte, um endlich ihre Meinung zum Umgang mit weiblicher Genitalbeschneidung in der Schweiz sagen zu können. In ihrem Interview benennt Nadifa Folgen des Kolonialismus, indem sie betont, dass sie die ständigen Einmischungen ,europäischer ${ }^{6}$ Bevölkerungsgruppen in die somalische ,Kultur ${ }^{6}$ nicht mehr ertragen könne. Ohne weibliche Genitalbeschneidung $\mathrm{zu}$ befürworten, verdeutlicht sie, woran es aus ihrer Sicht liegt, dass die Arbeit gegen weibliche Genitalbeschneidung - sowohl in Somalia als auch in der Schweiz - nur schleppend vorangeht.

Ayaans Interview wurde ausgewählt, weil sie die älteste Interviewpartnerin ist und in ruhiger, fast stoischer Art berichtet wie viele Schmerzen sie aufgrund ihrer Infibulation unter der Geburt ihrer fünf Kinder durch litten hat einen Schmerz, den sie als infibulierte Frauen hingenommen hat, der sie aber nicht am Kinder bekommen hindert. Ayaan ist Krankenschwester in England und kennt die medizinischen Folgen einer Infibulation. Sie weiß auch, wie infibulierte Frauen während der Geburt zu behandeln sind. Trotzdem berichtet sie davon, von Ärzt innen und Hebammen in England nicht ernst genommen worden zu sein.

Italien ist das einzige Land, in dem Interviews mit zwei Interviewpartnerinnen, mit Layla und Saida, analysiert wurden. Diese Entscheidung wurde getroffen, weil beide Interviews in großem Kontrast zueinander stehen, was nicht allein daran liegt, dass Layla sehr emotional von ihren vielschichtigen Problemen sprach, während Saida sich sehr distanziert zeigte und angab, keinerlei Schwierigkeiten mit ihrer Infibulation zu haben. Bei der Analyse des Interviews von Saida wurde deutlich, dass die Art der Interviewführung der Grund für ihre Distanz gewesen sein könnte. Das Interview mit Saida ist 
beispielhaft dafür, wie schnell ein Gespräch zu dieser sensiblen Thematik einen anderen Verlauf nimmt als erwünscht.

\subsection{Analyse der Interviews in Anlehnung an die Grounded Theory}

Die Auswertung der qualitativen Interviews mit den Frauen folgte dem Prinzip der Grounded Theory. Dies war aufgrund des explorativen Charakters der Untersuchung und den wenigen wissenschaftlichen Studien zur Perspektive beschnittener Frauen in Europa die passende Methode, da das Interviewmaterial als Ausgangspunkt für die daraus abgeleiteten theoretischen Erkenntnisse genutzt werden konnte.

Bei der Grounded Theory handelt es sich nicht nur um eine Analysemethode, vielmehr wird darunter der gesamte Forschungsprozess gefasst. Bereits während der Forschungsphase, wie auch während der Analyse des Materials, wird nach dem Prinzip des ständigen Vergleichens der Daten vorgegangen - ein Charakteristikum, das die Grounded Theory auszeichnet. Ein weiteres wichtiges Merkmal ist das so genannte ,theoretische Sampling“ (Glaser/Strauss 2008, S.61), worunter der ,auf die Generierung von Theorie zielende[ ] Prozess der Datenerhebung" (ebd.) gefasst wird. Ein Prozess, in dem gleichzeitig Daten erhoben, „kodiert und analysiert“ (ebd.) werden, so lange bis eine theoretische Sättigung erreicht ist. Das theoretische Sampling ist von dem statistischen Sampling zu unterscheiden. Während das theoretische Sampling darauf abzielt, „Kategorien und ihre Eigenschaften zu entdecken und das interne Beziehungsgefüge einer Theorie $\mathrm{zu}$ entwerfen" Glaser/Strauss 2008, S.78), wird das statistische Sampling genutzt, um „Individuen empirisch exakt auf für die Beschreibung und Verifizierung notwendiger Kategorien zu verteilen (Glaser/Strauss 2008, S.78). Der Prozess des theoretischen Sampling wird solange fortgeführt, bis eine ,theoretische Sättigung" (Strübin 2004, S.33) erreicht ist, worunter der Zeitpunkt in der Analyse fällt, an dem die Erhebung und Auswertung weiterer Daten „keine neuen Eigenschaften der Kategorie mehr erbringt und auch zu keiner Verfeinerung des Wissens um diese Kategorie mehr beiträgt" (ebd.).

Im Fall der in diesem Rahmen durchgeführten Untersuchung konnte nicht nach dem theoretischen Sampling vorgegangen werden, da es nicht möglich war, den Prozess der Datenerhebung zu steuern, weil die Auswahl der Interviewpartner_innen von den Kontaktpersonen abhängig war.

Die Analyse der Interviews erfolgte gemäß der Vorgangsweise der Grounded Theory, wonach die ausgewählten Interviews Wort für Wort gelesen und in Codes zerlegt wurden. Unter diesem so genannte Codieren wird „das Verschlüsseln oder Übersetzen von Daten“ (Böhm 2004, S. 476) verstanden. Ein 
Prozess, der ebenfalls der Methode des ständigen Vergleichs der Daten miteinander folgt und dazu dient, die erhobenen Daten auf „Unterschiede und Ähnlichkeiten" (Strübin 2004, S.18) zu untersuchen. Die Codes, die den Daten entnommen werden, beziehen sich noch sehr eng auf das Material, während die aus den Codes formulierten Kategorien etwas weiter gefasst sind. Nach der Formulierung bestimmter Kategorien, folgt die weitere Analyse derselben Vorgehensweise, dem Vergleichen der Kategorien miteinander (Glaser/Strauss 2008, S. 119). Das Vergleichen der Kategorien wird durch das Schreiben von so genannten Memos (Glaser/Strauss 2008, S. 121) unterbrochen. Die in den Memos festgehaltenen Gedanken sind notwendig, um die Ideen zu konkretisieren und noch einmal auf Schlüssigkeit zu prüfen. Dieser Prozess wurde außerdem von Diskussionen zu bestimmten Sequenzen aus den Interviews mit Mitdoktorand_innen unterstützt, die, wie Glaser und Strauss es formulieren, sehr dienlich sind, um „Verfehltes klarzustellen sowie Gesichtspunkte hinzuzufügen“ (ebd., S. 122). Im Rahmen dieser Diskussionen konnten die eigenen Ideen und Gedanken, die sich anhand der Kategorien entwickelt haben, noch einmal überprüft und durch Ideen aus anderen Denkrichtungen ergänzt werden. Die auftretenden Kategorien der einzelnen Interviews konnten auf diese Weise miteinander verglichen werden und auf Deutungs- oder Handlungsmuster sowie auf Wahrnehmung beziehungsweise Konstruktion der Problemlage hin untersucht werden.

Da der Fokus auf der Perspektive beschnittener Frauen lag, wurde während des Auswertungsprozesses der qualitativen Interviews entschieden, die Interviews mit den männlichen Interviewpartnern nicht nach der Grounded Theory auszuwerten, sondern in ihren zentralen Aussagen zusammenzufassen. Auf diese Weise wurde es möglich, alle geführten Interviews mit den Männern zu berücksichtigen und auf ihre zentralen Aspekte hinsichtlich weiblicher Genitalbeschneidung zu untersuchen und darzustellen. Da sie nicht den Eingriff nicht unmittelbar am eigenen Körper erlebt haben, ging es in den Interviews mit den männlichen Interviewpartnern weniger um ihr Erleben als mehr um ihre Sichtweise auf und den Umgang mit weiblicher Genitalbeschneidung. Die Zusammenfassung der zentralen Aussagen gibt diese gut wieder.

\subsection{Selbstreflexion als Forscherin}

Zum Abschluss soll nachfolgend noch auf meine Person als Forscherin eingegangen werden, da aus konstruktivistischer Perspektive keine objektive Forscherin existiert, weil „wir niemals die Dinge an sich, sondern nur die Dinge, so wie wir sie erleben, d.h. wie wir sie konstruieren, erkennen.“ (Muckel 1998, S.62). Folgt man der konstruktivistischen Perspektive existiert Objektivität nicht, da dies bedeuten würde, dass eine Wirklichkeit existiert, 
die von der sie beobachtenden Person losgelöst ist - eine Annahme, die kaum überprüfbar ist. Im Konstruktivismus wird davon ausgegangen, dass „wir ausschließlich Zugang zu einer von der Beobachterin abhängigen Wirklichkeit haben." (Muckel1998, S.63). Von dieser Annahme ausgehend ist der nachfolgende Blick auf meine Person als Forscherin und meine Konstruktionen von Wirklichkeit im Zusammenhang dieser Forschungsarbeit von Bedeutung.

So ist bereits die Wahl, Somalis zu interviewen, aufgrund meines eigenen somalischen Hintergrundes gefallen und meines Wunsches, ihre Meinung zum Thema weibliche Genitalbeschneidung zu erfassen. Mit dem Wissen, dass es sich um eine Bevölkerungsgruppe handelt, die an der schwersten Form genitaler Beschneidung festhält, hat sich das Interesse entwickelt, von den Menschen selbst zu hören, warum die Praktik ihrer Meinung nach weiter Bestand hat. Den Fokus auf die Somalis zu richten, die ihre Heimat verlassen haben und mittlerweile in einem europäischen Land leben, das weibliche Genitalbeschneidung ablehnt, erschien im Hinblick auf dieses Phänomen besonders interessant. Beschnittene somalische Frauen werden im gesellschaftlichen Kontext des Einwanderungslandes nicht allein aufgrund ihrer Hautfarbe oder nationalen Herkunft und Religion zu Minderheiten. Sie verkörpern außerdem noch eine Praktik, die im jeweiligen Einwanderungsland als Zeichen weiblicher Unterdrückung oder als Beispiel der Rückständigkeit ihrer Bevölkerungsgruppen gewertet wird. Dies wirft die Frage auf, ob die Erfahrung von Diskriminierung aufgrund der genannten Merkmale, sich dahingehend auswirkt, dass Angebote gegen Genitalbeschneidung nur schleppend angenommen werden, vielleicht sogar dazu beitragen eher an solchen Gepflogenheiten festzuhalten. Eine Frage, die sich aufgrund meiner persönlichen, teilweise diskriminierenden Erfahrungen als SchwarzeDeutsche in einer weißen Mehrheitsgesellschaft ergeben hat.

Auf der anderen Seite steht das Bild, das meine Interviewpartner_innen von mir haben und auf das sie reagieren. Unabhängig vom äußeren Erscheinungsbild, treffen sie auf eine Interviewperson, die in der deutschen Gesellschaft sozialisiert ist, von der sie wissen, dass sie Genitalbeschneidung ablehnt, die keine Verbindung zu Somalia hat und, westlich ' geprägt ist. Ihre Annahme, ich könnte ähnliche Bilder über Mädchenbeschneidung praktizierende Somalis haben, wie sie sie eventuell von ,Europäer_innen' kennen gelernt haben, kann eine gewisse Vorsicht mit sich bringen, vielleicht auch Zurückhaltung in Bezug auf ihre Meinung zum Thema Mädchenbeschneidung. Meine Position bringt somit zwar mit sich, dass ich auf der einen Seite Akzeptanz erfahre, gleichzeitig aber auch mit Distanz rechnen muss. Die Position, die ich verkörpere verändert sich je nach Blickwinkel, aus Sicht der weißen Mehrheitsgesellschaft oder aus dem Blickwinkel der somalischen Migrant_innen. Meine Perspektive als Forscherin auf die somalischen Interviewpartner_innen ist nicht losgelöst von meinen teilweise diskriminierenden 
Erfahrungen in einer weißen Mehrheitsgesellschaft. Dennoch darf die Vermutung, dass meine Interviewpartner_innen und ich in dieser Erfahrung verbunden sind nicht zu einem zu großen Gefühl der Verbundenheit meinerseits führen, da meine Interviewpartner innen ganz eigene Erfahrungen gesammelt und einen eigenen Umgang mit diesen gefunden haben können. 


\section{Ergebnisse der Befragung der Beratungsstellen}

Nachfolgend werden die Ergebnisse aus der quantitativen Untersuchung pro Einwanderungsland präsentiert und diskutiert. Die quantitative Erhebung richtete sich an Fachkräfte, die im Rahmen ihrer professionellen Tätigkeit in Einrichtungen und Kliniken gegen weibliche Genitalbeschneidung arbeiten. Diese quantitative Erhebung folgte der zentralen Frage, wie sich der professionelle Umgang mit von Genitalbeschneidung betroffenen und bedrohten Mädchen und Frauen von Seiten des in Einrichtungen und Kliniken tätigen Fachpersonals gestaltet. Nachfolgend werden die Ergebnisse dieser Erhebung präsentiert. Die offenen Fragen des teilstandardisierten Fragebogens fließen in die Auswertung ein, sind aber grundsätzlich als offene Fragen gekennzeichnet. Im Anschluss an die länderspezifische Auswertung folgt die $\mathrm{Zu}$ sammenfassung der zentralen Aussagen.

\subsection{Zur Beratungssituation in Deutschland}

Von 15 deutschen Beratungsstellen haben zehn Einrichtungen geantwortet, wovon die große Mehrheit der Einrichtungen bereits länger als zehn Jahre existiert. Die Mehrzahl der den Fragebogen beantwortenden Mitarbeiter_innen gibt an, sich mindestens fünf Jahre mit weiblicher Genitalbeschneidung beschäftigt zu haben. Auf die Fragen, ob Personen aus afrikanischen Ländern und von Genitalbeschneidung betroffene Frauen in den Einrichtungen tätig seien, geben sieben an, dass Menschen afrikanischer Herkunft in ihrer Einrichtung arbeiten und fünf davon sagen, dass darunter auch betroffene Frauen sind. Acht Einrichtungen stimmen der Aussage zu, dass es von Bedeutung ist, betroffene Frauen mitarbeiten zu lassen, da diese bedrohte und betroffene Mädchen und Frauen besser erreichen könnten und dies vor allem die Möglichkeit biete, Beratungsgespräche in der Muttersprache durchzuführen. Alle Einrichtungen stimmen mit der Antwort überein, dass mit dem Einsatz betroffener Frauen in der Beratungsarbeit gegen Genitalbeschneidung Missverständnissen vorgebeugt werden könne und es für bedrohte und betroffene Mädchen und Frauen die Hemmschwelle herabsetze, wenn selbst betroffene Frauen in der Beratungsstelle tätig seien. Der Fokus deutscher Beratungsstellen liegt auf bedrohten und betroffenen Mädchen und Frauen, aber zwei Drittel geben an, auch das gesamte Umfeld der Mädchen und Frauen in ihre Beratungsarbeit einzubeziehen. Auch die Aufklärung und Beratung für Klinikpersonal, freie Hebammen, Pädagog_innen, und Politiker_innen im 
Umgang mit betroffenen Frauen führt die Mehrheit der Einrichtungen durch. Schwierig scheint dagegen, bedrohte Mädchen zu erreichen: die Frage, wie gut sie bedrohte und betroffene Mädchen und Frauen erreichen würden, beantworten nur zwei Einrichtungen mit sehr gut beziehungsweise gut. Die restlichen sieben geben an, dass dies eher schwierig bis sehr schwierig sei. Betroffene Frauen dagegen würden vorwiegend von selbst in die Einrichtung kommen und Angebote wahrnehmen. Um dennoch bedrohte Mädchen zu erreichen, führen einige deutsche Einrichtungen Projekte durch, die sich an das gesamte familiäre Umfeld dieser Mädchen richten. In diesen beiden offenen Fragen geben manche an, die gesamte Familie mit Beratungsgesprächen zum Thema zu erreichen. Andere binden bedrohte Mädchen in Projekte ein, in denen Mädchenbeschneidung künstlerisch thematisiert wird, wie beispielsweise in einem Theaterprojekt oder mithilfe speziell ausgerichteter Comics. Dieser Ansatz habe sich besonders bewährt, da auf diese Weise sowohl Mädchen und Jungen erreicht werden könnten. Eine Einrichtung berichtet, dass sich ihre neu ins Leben gerufene Telefonberatung als sinnvoll erweise, da diese besonders in der Öffentlichkeit das Bewusstsein für Mädchenbeschneidung schärfe. Aus den Angaben der Einrichtungen geht allerdings hervor, dass die Zielgruppe der Jungen und Männer in der Arbeit gegen Mädchenbeschneidung nur wenig Beachtung findet: Nur ein Drittel der befragten Einrichtungen führt Angebote für Jungen und Männer durch.

Die konkreten Angebote für betroffene Mädchen und Frauen orientieren sich an der Bedürfnislage der Mädchen und Frauen und gestalten sich daher ganz unterschiedlich. Die Mehrheit begleitet die Frauen sozialpädagogisch, medizinisch und/oder psychologisch, vereinzelt werden sie aber auch an Mediziner_innen und/oder Psycholog_innen weitervermittelt. Doch nur wenige Einrichtungen beziehen das gesamte Umfeld betroffener Mädchen und Frauen mit ein. Alle Einrichtungen sind aber der Meinung, Bedrohte und Betroffene mittlerweile besser zu erreichen als noch zu Anfangszeiten ihrer Arbeit, was daran liege, dass sich ihre Angebote innerhalb der Communities herumsprechen würden.

Zum Zeitpunkt der Befragung (2011/12) bestand noch kein explizites Gesetz gegen weibliche Genitalbeschneidung in Deutschland, die Praktik fiel unter den Straftatbestand der Körperverletzung beziehungsweise schweren Körperverletzung. Auf die Frage, ob die bestehende Gesetzeslage in der Arbeit gegen Mädchenbeschneidung hilfreich sei, bejahten dies dennoch alle Einrichtungen. Gleichzeitig gab nur eine Einrichtung an, schon einmal eine Familie angezeigt zu haben, die den Eingriff für ihre Tochter plante.

Im letzten Teil des Fragebogens wurde die Zusammenarbeit der verschiedenen Einrichtungen mit Gynäkolog_innen und Psycholog_innen thematisiert. Aus diesem Block kann festgehalten werden, dass alle Einrichtungen die Zusammenarbeit mit diesen beiden Berufsgruppen als wichtig erachten 
und die Mehrheit der Einrichtungen auch Schulungen im Umgang mit beschnittenen Mädchen und Frauen anbietet, um genannten Berufsgruppen für die Thematik zu sensibilisieren. Es sind auch alle Einrichtungen der Meinung, dass Schulungen für genannte Berufsgruppen wichtig sind und deshalb forciert werden sollten. Obwohl alle Einrichtungen angeben, im Kontakt mit erfahrenen Gynäkolog_innen, teilweise auch mit erfahrenen Psycholog_innen zu stehen, schätzt die Mehrheit der Einrichtungen die Zusammenarbeit mit diesen Berufsgruppen weniger gut ein.

Im offenen Teil dieses Abschnitts haben manche Einrichtungen angemerkt, dass es förderlich wäre das Thema Mädchenbeschneidung in die Aus- und Weiterbildung dieser beiden Berufsgruppen aufzunehmen. Des Weiteren sollten mehr zeitliche und finanzielle Ressourcen zur Verfügung stehen, um regelmäßige Fortbildungen für genannte Berufsgruppen durchführen zu können. Eine Einrichtung betont, dass es wichtig sei, das Interesse für das Thema zu wecken, da sie die Erfahrung gemacht hätten, dass die Zusammenarbeit mit Ärzt_innen und Psycholog_innen, die Interesse am Thema hätten, am effektivsten sei.

\subsection{Zur Beratungssituation in der Schweiz}

In der Schweiz existieren 25 Einrichtungen, die gegen Mädchenbeschneidung tätig sind, sieben haben den Bogen ausgefüllt zurückgesendet. Alle Einrichtungen existieren länger als fünf, die große Mehrheit länger als zehn Jahre. Die den Bogen beantwortenden Personen beschäftigen sich meist länger als fünf, häufig auch länger als zehn Jahre mit dem Thema Mädchenbeschneidung. Die Mehrheit der Einrichtungen gibt an, dass sie sowohl Männer als auch Frauen afrikanischer Herkunft beschäftigen, darunter auch von weiblicher Beschneidung betroffene Frauen. Alle sind der Meinung, dass selbst betroffene Frauen in der Arbeit gegen Mädchenbeschneidung wichtig sind, da sie Betroffene und deren Familien besser erreichen und Beratungs- und Aufklärungsarbeit so in der Muttersprache stattfinden können. Die Angebote der schweizerischen Einrichtungen richten sich mehrheitlich an von Genitalbeschneidung betroffene Frauen, weniger an Jungen und Männer. Der Schwerpunkt liegt auf der Beratung von erwachsenen Frauen im Umgang mit weiblicher Genitalbeschneidung, sowie der Begleitung bedrohter Mädchen. Das gesamte soziale Umfeld bedrohter und betroffener Mädchen und Frauen wird von drei Einrichtungen in die Aufklärungsarbeit mit einbezogen. Die Mehrheit der Einrichtungen gibt an, vor allem Informationsveranstaltungen für medizinisches und pädagogisches Fachpersonal zum Thema durchzuführen, um diese Berufsgruppen zu sensibilisieren. Wie auch in Deutschland erreichen die schweizerischen Einrichtungen bedrohte und betroffene Mäd- 
chen mit ihren Angeboten nur schwer, wohingegen betroffene Frauen gut erreicht werden. Auf die beiden offenen Fragen zur Gestaltung der Präventionsangebote für Mädchen und deren sozialen Umfelder gibt eine Einrichtung an, vor allem Informationsgespräche mit bedrohten Mädchen und deren Eltern zu führen. Diese richten sich besonders an die weiblichen Bezugspersonen, also Mütter, Großmütter, Tanten, werdende Mütter, und finden meist im familiären Umfeld der Mädchen statt. Diese und eine weitere Einrichtung betonen die Wichtigkeit von Kulturvermittler_innen, die sie zu diesen Gesprächen grundsätzlich hinzuziehen würden. Eine Einrichtung nutzt Geburtsvorbereitungskurse, um sowohl den werdenden Müttern als auch deren Partnern die Folgen von Mädchenbeschneidung näher zu bringen - wenn nötig würden diese Gespräche auch mit einer/m Übersetzer_in stattfinden. Neben diesen Informationsgesprächen, berichtet eine andere Einrichtung, dass sie die Mädchen am besten über attraktive Freizeitangebote wie Filmvorführungen, Musikabende und gemeinsamem Essen erreicht könnten. Einig sind sich die Einrichtungen, die den offenen Frageteil beantwortet haben, darin, dass besonders wichtig sei, die enge, partnerschaftliche Zusammenarbeit mit den Communities zu fördern, um bedrohte Mädchen schützen zu können. Trotz der vielfältigen Angebote handle es sich bei der Gruppe bedrohter Mädchen um eine eher schwierig zu erreichende Zielgruppe. Dennoch geben die schweizerischen Einrichtungen an, dass ihre Angebote mittlerweile von bedrohten und betroffenen Mädchen und Frauen besser angenommen würde als noch zu Anfangszeiten. Dies liege daran, dass sich ihre Angebote in den Communities herumgesprochen haben, was alle Einrichtungen als vorteilhaft bewerten.

In Bezug auf die männlichen Familienmitglieder bedrohter und betroffener Mädchen, geben zwei Einrichtungen an, präventive Angebote für Jungen und Männer durchzuführen. Dies seien dann Informationsveranstaltungen oder spezielle Seminare, zu denen beispielsweise religiöse Führer und Sexualpädagog_innen eingeladen und mit den Jungen und Männern über weibliche Genitalbeschneidung diskutieren würden. Es wären auch schon Abende durchgeführt worden, an denen betroffene Frauen über die Folgen der Praktik berichteten, mit dem Ziel den Jungen und Männern die negativen Folgen zu verdeutlichen.

Im Hinblick auf die konkreten Angebote für Betroffene variieren die Einrichtungen stark in der Form der Begleitung betroffener Frauen: Manche begleiten ausschließlich sozialpädagogisch, manche medizinisch, manche psychologisch. Eine Einrichtung gibt an, alle diese Bereiche der fachlichen Begleitung der Frauen abzudecken. Die Gesetzgebung in der Schweiz empfinden alle bis auf eine Einrichtung als hilfreich in ihrer Arbeit gegen Mädchenbeschneidung. Doch, wie auch in Deutschland, gibt nur eine Einrichtung an, einmal eine Familie angezeigt zu haben, die ihre Tochter dem Eingriff unterziehen wollte. 
Zu Gynäkolog_innen, die sich mit den Hintergründen und den Konsequenzen genitaler Beschneidung auskennen, haben alle Einrichtungen Kontakt, zu erfahrenen Psycholog_innen vier Einrichtungen. Vermittlungen betroffener Frauen zu erfahrenen Gynäkolog_innen finden in vier Einrichtungen sehr oft bis oft statt, zu Psycholog_innen weniger oft bis nie. Die Hälfte der Einrichtungen bewertet die Zusammenarbeit mit Gynäkolog_innen und Psycholog_innen, die Erfahrung im Umgang mit beschnittenen Frauen haben, als gut. Im offenen Teil äußert eine Einrichtung, dass sie sich mehr Kontakt zu diesen Berufsgruppen wünschen würde. Alle Einrichtungen sind der Meinung, dass Schulungen für beide Berufsgruppen sehr wichtig sind. Die Mehrheit führt Schulungen für Mediziner_innen (Gynäkolog_innen, Kinderärzte und Kinderärztinnen) und Psycholog_innen selbst durch.

\subsection{Zur Beratungssituation in England}

In England existieren 26 Einrichtungen, von denen sieben geantwortet haben. Alle sieben existieren bereits länger als fünf Jahre, drei Einrichtungen geben an, länger als zehn Jahre gegen Genitalbeschneidung zu arbeiten. Die Personen, die den Fragebogen ausgefüllt haben, beschäftigen sich meist länger als fünf, häufig bereits länger als zehn Jahre mit dem Thema Mädchenbeschneidung. Alle Einrichtungen beschäftigen beschnittene Frauen. Die Mehrheit der Befragten gibt an, die Anstellung selbst betroffener Frauen als Vorteil zu betrachten, da dieser Umstand helfe, beschnittene Frauen zu erreichen, und so Aufklärungsarbeit gegen Beschneidung in der Muttersprache stattfinden könnte. Außerdem hätten Betroffene weniger Hemmung, eine Beratung in Anspruch zu nehmen, wenn sie wüssten, dass ebenfalls Betroffene dort arbeiten würden.

Auch in den britischen Einrichtungen steht die Begleitung und Beratung von Genitalbeschneidung bedrohter Mädchen und betroffener Frauen im Mittelpunkt. Weniger Beachtung finden dabei die Jungen und Männer und/oder das soziale Umfeld betroffener Mädchen und Frauen. Die große Mehrheit gibt noch an, Öffentlichkeitsarbeit zu Mädchenbeschneidung sowie Informationsveranstaltungen für Klinikpersonal durchzuführen. So wie auch in Deutschland und der Schweiz gibt die Mehrheit der britischen Einrichtungen an, Mädchen nur schwer erreichen zu können. Bei den betroffenen Frauen gestaltet sich dies etwas einfacher: fünf Einrichtungen erreichen diese Zielgruppe sehr gut bis gut und nur zwei eher schwierig. Die Frauen würden die Angebote von selbst nutzen und in die Einrichtung kommen. Im offenen Teil haben einige Einrichtungen die Gestaltung ihrer Angebote für Mädchen und deren Umfeld konkretisiert. Manche führen Workshops mit den Mädchen und Frauen durch, in denen neben der Erläuterung der Konsequenzen 
von Mädchenbeschneidung auch stark auf die in England bestehende Gesetzeslage eingegangen wird. Den Mädchen werde in diesen Informationsgesprächen auch vermittelt, welche Wege sie gehen können, um sich und andere vor der Praktik zu schützen. Weiterhin werde zum Schutz der Mädchen eng mit den praktizierenden Communities gearbeitet, um möglichst viele Mitglieder aus dem Umfeld der Mädchen über die Folgen und die Gesetzeslage in England aufzuklären. Eine Einrichtung berichtet von einem sehr erfolgreichen TV-Spot zum Thema Mädchenbeschneidung, den sie speziell für einen somalischen Fernsehsender aufgenommen hatte. In somalischer Sprache würden die Mädchen und Frauen darin auf ihre Klinik und die Möglichkeiten im Umgang mit Beschneidung aufmerksam gemacht. Seitdem würden sich sehr viele Mädchen aus dem In- und Ausland bei ihnen melden, um sich beraten zu lassen, viele würden auch den nächsten Schritt gehen und ihre Infibulation öffnen lassen.

Alle Einrichtungen geben an, dass sich ihre Arbeit mittlerweile innerhalb der Communities im Vergleich zu Anfangszeiten herum gesprochen habe, was alle als vorteilhaft empfinden. Die Angebote für betroffene Mädchen und Frauen gestalten sich in allen Einrichtungen ähnlich: Sie bieten medizinische Unterstützung für betroffene Frauen, zwei bieten darüber hinaus psychologische Begleitung an. Besteht der Verdacht, dass ein Mädchen im familiären Umfeld der betroffenen Frau beschnitten werden soll, beziehen drei Einrichtungen auch das Umfeld in die Aufklärungsarbeit mit ein, ansonsten findet das familiäre Umfeld weniger Berücksichtigung. Die nationale Gesetzgebung wird von allen Einrichtungen in der Arbeit gegen Mädchenbeschneidung als hilfreich empfunden, doch auch in England gibt nur eine Einrichtung an, schon einmal eine Familie angezeigt zu haben.

Auf die offene Frage bezüglich der Einbindung der Jungen und Männer in die Aufklärungsarbeit berichtet eine Einrichtung, dass sie diese Zielgruppe vor allem über die enge Zusammenarbeit mit den Communities erreichen würde. Aus dieser Zusammenarbeit entstünden Projekte und Angebote, die sich gezielt an die männlichen Mitglieder richten und mittlerweile auch regelmäßig stattfinden würden. Dennoch findet diese Zielgruppe auch in England wenig Beachtung.

Alle Einrichtungen haben Kontakt zu Gynäkolog_innen, die sich mit Mädchenbeschneidung auskennen, vier pflegen diesen Kontakt auch zu Psycholog_innen. Vermittlungen zu informierten Gynäkolog_innen und Psycho$\log$ innen finden nur vereinzelt statt. Die Vermittlung betroffener Mädchen und Frauen von Gynäkolog_innen zu Einrichtungen, die gegen Genitalbeschneidung arbeiten, finde nur manchmal statt, doch weniger häufig passiere es, dass Psycholog_innen Betroffene vermitteln würden. Schulungen im Umgang mit beschnittenen Mädchen und Frauen sollten nach Meinung aller Einrichtung für diese beiden Berufsgruppen stattfinden, vier Einrichtungen führen solche Schulungen selbst durch. Die überwiegende Mehrheit der Ein- 
richtungen gibt an, dass die Zusammenarbeit mit den Gynäkolog_innen und Psycholog_innen nicht gut sei, doch nur eine äußert sich im offenen Teil dazu, wie dieser Kontakt verbessert werden könnte. Darin betont diese, wie wichtig es sei, bei Gynäkolog innen ein größeres Bewusstsein für die Thematik zu schaffen. So könne man künftig auch erreichen, dass früher auf beschnittene Frauen reagiert werden könne, nicht erst während der Geburt, sondern bereits vor oder während der Schwangerschaft.

Eine Mitarbeiterin einer Einrichtung, die bereits seit 25 Jahren gegen Mädchenbeschneidung arbeitet, schreibt im offenen Teil, dass die letzten Jahre gezeigt hätten wie wichtig der verständnisvolle und aufsuchende Kontakt zu praktizierenden Communities sei. In diesen Gesprächen müsse unbedingt darauf verwiesen werden, dass Mädchenbeschneidung in England verboten ist, da etliche Familien dies nicht wüssten. Aus Sicht dieser Mitarbeiterin seien die Communities der beste Ort, um die Familien bedrohter und betroffener Frauen und Mädchen zu erreichen und einen Wandel in der Haltung gegenüber Beschneidung herbeizuführen, um künftig Mädchen vor der Praktik zu schützen. Aus ihrer Sicht sei ein weiterer wichtiger Aspekt in der Arbeit gegen Mädchenbeschneidung die Vernetzung der verschiedenen Einrichtungen untereinander, nicht nur innerhalb Englands, sondern europaweit. So könne im Austausch voneinander gelernt und Angebote spezifiziert und verbessert werden.

\subsection{Zur Beratungssituation in Italien}

In Italien haben sich von sechs Einrichtungen drei an der Umfrage beteiligt. Alle drei Einrichtungen existieren bereits länger als zehn Jahre und auch die den Bogen beantwortenden Personen sind bereits länger als zehn Jahre in der Einrichtung tätig und beschäftigen sich mehr als zehn Jahre mit dem Thema weibliche Genitalbeschneidung. In einer Einrichtungen arbeiten sowohl Männer als auch Frauen afrikanischen Hintergrundes und selbst von Beschneidung Betroffene. Alle stimmen den Aussagen zu, dass es wichtig ist, betroffene Frauen in ihrer Einrichtung zu beschäftigen, da sie andere Betroffene besser erreichen, Beratungsgespräche in der Muttersprache stattfinden und auf diese Weise Missverständnisse vermieden werden könnten. Die Einrichtungen legen den Schwerpunkt ihrer Arbeit auf die Beratung und Begleitung bedrohter und betroffener Mädchen und Frauen. In einer Einrichtung existieren ebenfalls Angebote für Jungen und Männer. Alle Einrichtungen informieren zudem Klinikpersonal zur Thematik, zwei auch Pädagog_innen, Jurist_innen, Politiker_innen und die breite Öffentlichkeit.

Zwei Einrichtüngen geben an, sowohl bedrohte Mädchen als auch betroffene Mädchen und Frauen mit ihren Angeboten gut zu erreichen. $\mathrm{Zu}$ den 
Familien der Betroffenen pflegt eine Einrichtung Kontakt. Alle sind der Meinung, dass sie mittlerweile mehr Bedrohte und Betroffene erreichen als noch zu Anfangszeiten ihrer Arbeit. Die nationale Gesetzgebung empfinden zwei Einrichtung als Vorteil in der Arbeit gegen Beschneidung, doch hat bislang keine Einrichtung einen Fall zur Anzeige gebracht. Die Arbeit habe sich aber mittlerweile in den Communities herum gesprochen, so geben dies zwei Einrichtungen an, die dies auch als Vorteil empfinden. Die Beratung und Begleitung betroffener Mädchen und Frauen gestaltet sich in einer Einrichtung vielseitig: Betroffene werden sowohl sozialpädagogisch, medizinisch als auch psychologisch beraten und begleitet.

Alle Einrichtungen haben guten Kontakt zu Gynäkolog_innen und Psycholog_innen, die sich mit den Hintergründen und Konsequenzen weiblicher Beschneidung auskennen. Insgesamt würden sie Betroffene aber nur vereinzelt an Gynäkolog_innen und Psycholog_innen vermitteln, ähnlich verhalte es sich auch andersherum: vereinzelt kämen betroffene Frauen von Gynäkolog_innen und Psycholog_innen zu ihnen in die Einrichtung. Alle Einrichtungen geben an, Schulungen für diese beiden Berufsgruppen für wichtig zu empfinden und alle bieten diese Schulungen auch an. Die Einrichtungen schätzen die Zusammenarbeit mit den Gynäkolog_innen und Psycholog_innen als gut ein.

In den offenen Fragen betont eine Einrichtung, dass es wichtig sei, Betroffenen gegenüber respektvoll zu begegnen. Wenn die Frauen ihre Einrichtung verlassen und das Gefühl hatten, respektvoll behandelt worden zu sein, kämen sie wieder, was sich auch in ihrem sozialen Umfeld herumspreche und daher vorteilhaft für die Arbeit gegen weibliche Genitalbeschneidung sei. Eine andere Einrichtung berichtet, dass sie es als besonders wertvoll wahrnehme, einen somalischen Chefarzt an der Klinik zu haben, da dieser nicht nur bei den Frauen zu einem offenen Zugang beitragen könne, sondern auch das Gespräch mit den somalischen Männern suchen würde. Des Weiteren würde der somalische Arzt Kontakte zu Moscheen und den Imamen herstellen, um sie dafür zu gewinnen, die Menschen darüber aufzuklären, dass weibliche Genitalbeschneidung keine religiöse Pflicht darstelle. Laut der Einrichtung sei dies ebenfalls ein Ansatz, der sich bewährt habe, da die Mehrheit der Menschen es als religiöse Pflicht verstünden, ihre Töchter zu beschneiden. Die Einrichtungen betonen außerdem die Wichtigkeit der Vernetzung sowohl auf nationaler wie auch internationaler Ebene.

\subsection{Zentrale Ergebnisse der Befragung}

Die Umfrage zeigt deutlich, dass weibliche Genitalbeschneidung längst kein Randthema mehr ist, dem sich ein paar wenige Einrichtungen ange- 
nommen haben. In allen Befragungsländern existiert mittlerweile eine Vielzahl an Einrichtungen, die sich seit vielen Jahren gegen Mädchenbeschneidung engagieren. Die den Fragebogen beantwortenden Personen sind allesamt entweder als Leitung der Einrichtung tätig oder hauptamtlich Tätige und beschäftigen sich ebenfalls seit etlichen Jahren mit der Thematik. Somit kann festgehalten werden, dass die teilstandardisierten Fragebögen von Personen ausgefüllt wurden, die nicht nur viel Hintergrundwissen mitbringen, sondern ebenfalls die Entwicklungen der letzten Jahre (manche sogar Jahrzehnte) mitbekommen haben und eventuell maßgeblich daran beteiligt waren.

Die dem Fragebogen zugrunde liegende erste Vermutung, dass sich die Beschäftigung von Personen afrikanischer Herkunft, die aus Mädchenbeschneidung praktizierenden Bevölkerungsgruppen kommen, bewährt, ist bestätigt worden. Die überwiegende Mehrheit der Einrichtungen beschäftigt Männer und Frauen afrikanischer Herkunft, worunter auch selbst Betroffene $\mathrm{zu}$ finden sind. Alle Einrichtungen waren der Meinung, dass ihnen dies den Zugang zu weiteren betroffenen Mädchen und Frauen vereinfachen würde. Die Tatsache, dass auf diese Weise Beratungs- und Aufklärungsgespräche in der Muttersprache stattfinden könnten, betrachtet die Mehrheit der Befragten als positiv. Auf die Frage, wie gut die Einrichtungen bedrohte Mädchen mit ihren Angeboten erreichen, gibt die Mehrzahl der Einrichtungen an, dass es die am schwersten zu erreichende Zielgruppe sei. Im Vergleich zu betroffenen Frauen, die von den Angeboten nach Einschätzung der Einrichtungen gut profitieren. Dies liegt vermutlich daran, dass Mädchen noch stark unter dem Einfluss ihrer Eltern stehen, Angebote demnach so konstruiert sein müssten, dass Eltern ihren Töchtern die Erlaubnis zur Teilnahme an diesen erteilen. An der Zielgruppe der Mädchen zeigt sich deutlich, wie wichtig es ist, das gesamte Umfeld Bedrohter und Betroffener einzubeziehen. Diesbezüglich erscheint auch die Community von großer Bedeutung, da viele Familien Teil einer Community sind und diese Gemeinschaft mehr genutzt werden sollte, um mehr Eltern und Mädchen über die Konsequenzen, aber auch die gesetzliche Lage im jeweiligen Einwanderungsland aufzuklären.

Die zweite Vermutung war, dass Jungen und Männer in der Arbeit gegen Mädchenbeschneidung vernachlässigt werden. Auch diese Vermutung wurde durch Umfrage bestätigt: Die wenigsten Einrichtungen geben an, Angebote für Jungen und Männer zu haben, obwohl sie die Wichtigkeit dieser Angebote bestätigen. Die Einrichtungen, die auch den offenen Frageteil beantwortet haben, betonen, dass sich ihre Arbeit eng an den praktizierenden Communities orientiert - was Jungen und Männer ebenfalls mit einschließt. Ähnlich wie bei den minderjährigen Mädchen sind auch die Jungen und Männer entweder über ihr familiäres Umfeld erreichbar oder über die Community. Community-orientierte Arbeit kann daher in der Arbeit gegen Mädchenbeschneidung als zentraler Bestandteil festgehalten werden. 
Nahezu alle Einrichtungen empfinden die nationale Gesetzgebung in der Arbeit gegen Mädchenbeschneidung als hilfreich, auch die deutschen Einrichtungen, die sich zum Zeitpunkt der Befragung noch nicht auf ein explizites gesetzliches Verbot der Mädchenbeschneidung stützen konnten. Nur vereinzelt kam es jedoch zu Anzeigen von Familien, die planten, ihre Mädchen beschneiden zu lassen. Da Untersuchungen ergeben haben, dass Mädchen auch in Europa nicht vor Beschneidung geschützt sind, weil sie entweder im jeweiligen Einwanderungsland beschnitten oder zurück ins Heimatland gebracht werden, ist der Schutz bedrohter Mädchen per Gesetz wichtig. Warum dies jedoch bislang nur wenig verfolgt und angezeigt wird, liegt vermutlich an vielen Faktoren: Uninformiertheit auf Seiten der Behörden (Polizei, Richter_innen, Jugendamt), zu wenig Bewusstsein unter Kinderärzt_innen und zu wenig Kontrolle und/oder Handhabe, wenn die Mädchen in ihrem Heimatland beschnitten werden. Die strafrechtliche Verfolgung von Mädchenbeschneidung ist erst sinnvoll, wenn die Menschen über das bestehende Gesetz informiert wurden. Dann aber sollte auch sichergestellt sein, dass das Gesetz nicht nur auf dem Papier besteht, sondern falls erforderlich auch Anwendung findet.

Die Zusammenarbeit mit niedergelassenen Gynäkolog_innen und Psycholog_innen wird, außer in Italien, meist weniger gut eingeschätzt, womit sich die letzte Vermutung ebenfalls bestätigt. Der Hinweis einiger Einrichtungen, dass es wichtig wäre die Thematik in die Aus- und Weiterbildung der beiden Berufsgruppen aufzunehmen, könnte sicher dazu beitragen, ein größeres Bewusstsein und Interesse zu schaffen. Möglich wäre sicher auch, die erfahrenen Gynäkolog_innen und Psycholog_innen zur Gewinnung weiterer Kolleg_innen zu nutzen, um so für jede Region Ansprechpartner_innen zu haben, die Betroffene ohne zu weite Anfahrtswege nutzen können. 


\section{Ergebnisse der migrierten Frauen somalischer Herkunft}

Nachfolgend erfolgt die Darstellung der Analysen der Interviews mit den somalischen Frauen. Beginnend mit Sara, der Interviewpartnerin aus Deutschland, deren Interview deutlich macht wie viel Ambivalenz die Migration in ein Genitalbeschneidung ablehnendes Einwanderungsland mit sich bringen kann. Das zweite Interview mit Nadifa zeigt, dass die Arbeit gegen weibliche Genitalbeschneidung und der Umgang mit Betroffenen teilweise als überheblich und postkolonial wahrgenommen werden. Ayaan, die dritte Interviewpartnerin aus England wiederum spricht ausschließlich von den medizinischen Konsequenzen des Eingriffs, die sie aus eigener Erfahrung kennt und deshalb auch der Meinung ist, dass in erster Linie diesen begegnet werden muss. Den Abschluss bilden die beiden sehr gegensätzlichen Interviews aus Italien: Layla und Saida. Diese verdeutlichen zum einen die große Verzweiflung einer beschnittenen, geflohenen Frau und zum anderen, dass eine Genitalbeschneidung problematisierende Haltung zu Distanz und Ablehnung bei Betroffenen führen kann.

\subsection{Sara}

Sara wurde in einer großen Stadt in Somalia geboren und hat dort bis zur siebten Klasse die Schule besucht. Danach musste sie ihrer Mutter im Haushalt helfen. Im Alter von 17 Jahren verließ sie Somalia, um ihren bereits in Deutschland lebenden Mann zu heiraten. Diesen hatte Saras Familie noch bevor er nach Deutschland ausgewandert ist für sie ausgesucht. Seit 1990 leben sie gemeinsam in Deutschland und haben drei Kinder, die mittlerweile alle im jungen Erwachsenenalter sind. Zum Zeitpunkt des Interviews ist Sara Ende 30. Sowohl Sara als auch ihr Ehemann waren bereit zum Thema weibliche Genitalbeschneidung interviewt zu werden.

Das Interview findet bei Sara zuhause statt, wo auch noch ihr Ehemann, ihre Tochter und einer ihrer Söhne anwesend sind. Während die beiden Männer sich in einem anderen Raum aufhalten, sitzt Saras Tochter während des Interviews als Zuhörerin dabei. Einmal redet sie kurz mit, allerdings handelt es sich dabei um einen Abschnitt im Interview, der bei der Rekonstruktion nicht berücksichtigt wurde. Sara gibt vor dem Interview an, dass sie kein Problem damit habe, wenn ihre Tochter zuhöre, da sie mit ihr auch schon über Genitalbeschneidung und ihre Erfahrungen mit der Praktik gesprochen habe. Es entsteht der Eindruck, dass Sara es als unterstützend empfindet ihre 
Tochter neben sich zu haben, was sich in kleinen Gesten zeigt, wie dass sie sich gegenseitig an der Hand halten.

Sara erzählt nicht spontan von ihrem Leben in Somalia, sie gibt an Hemmungen zu haben, weil sie ihr Deutsch als nicht ausreichend für Erzählungen empfindet. Daher sind mehr konkrete Nachfragen nötig, die Sara aber dann doch dazu bringen, ein wenig zu erzählen. Auf die Frage, wie ihr Leben in Somalia war, beginnt Sara nicht chronologisch mit den ersten Erinnerungen an ihre Kindheit. Diese spart sie erst einmal aus und beginnt die Erzählung mit ihrer Jugend.

S: „Oh, also Jugend war halt Hausmädchen. Man hat halt nicht so viele Freiheiten, ab dem 13. Lebensjahre darf man halt nicht so viel wie jetzt die europäischen Frauen: frei sein, Kino gehen, man musste zuhause bleiben, im Haushalt den Eltern helfen und ab 15 oder ab 16 bist du verlobt. Die Eltern sagen dann zu dir: Jetzt ist Zeit, du bist erwachsen geworden, du bist reifer und es ist nicht schön immer hier zuhause zu bleiben. Irgendwann musst du heiraten. Ich habe auch geheiratet, halt den Mann, den sie ausgesucht haben. Die Eltern schreiben dir halt was vor und du musst halt ,ja“" sagen." (I 1, 3339). ${ }^{16}$

Sara lässt ihre Jugend mit dem 13. Lebensjahr beginnen. Sie erzählt, dass somalische Mädchen mit Beginn der Jugend „Hausmädchen“ seien, die nur wenige Freiheiten hätten. Für Sara ist mangelnde Freiheit vor allem mit zuhause bleiben und nicht ausgehen dürfen verbunden. Relativ schnell würde dann die von den Eltern arrangierte Heirat der Mädchen folgen, so Sara. Sara spricht von „Hausmädchen“ und Mädchen, die verheiratet werden, die männlichen Jugendlichen spielen in ihrer Erzählung keine Rolle.

Sara berichtet von einer aus ihrer Sicht sehr eingeschränkten Jugendzeit, die von viel Hausarbeit und Verboten geprägt war. Aus Saras Erzählung ist zu entnehmen, dass sie die Freiheitseinschränkungen mit ihrem Geschlecht in Verbindung bringt: Sie als Mädchen war ab dann ,halt Hausmädchen“ und musste einen Mann heiraten, den die Eltern vorschlugen. Hinzu kommt, dass Sara den Blick auf ihre Jugend aus heutiger Sicht bewertet. Aus diesem Blickwinkel betrachtet, vergleicht sie das heutige Leben ,europäischer Frauen" mit ihrem damaligen Leben als dreizehnjähriges Mädchen in Somalia. Sara spricht durchgehend von „Europa“ oder „europäisch“, verwendet diese Begrifflichkeiten jedoch immer, wenn sie von Deutschland oder deutschen Mädchen/Frauen/Männern spricht, was den Schluss nahe legt, dass sie das deutsche Leben meint. Aus diesem Vergleich ergibt sich für sie die Schlussfolgerung, weniger Freiheiten gehabt zu haben als „europäische Frauen“. Sie durfte nicht selbständig entscheiden, ob sie ausgehen oder wen sie heiraten wollte. Dies wurde über ihren Kopf hinweg entschieden, während sie nur 
machen konnte was ihre Eltern für richtig hielten, bis sie schließlich mit 17 Jahren den Mann heiratet, den die Eltern für sie ausgesucht hatten.

Sara berichtet nüchtern von ihrer Jugend, dennoch bleibt der Eindruck bestehen, dass sie es bedauert, nicht wie die ,europäischen Frauen“ aufgewachsen zu sein, da deren Leben für Sara mit Zuschreibungen wie ,Freiheiten " und ,Wahlmöglichkeiten' verbunden ist. Der etwas nüchtern vorgetragene Bericht zu ihrer Jugend, bleibt daher nicht frei von Wertung. Die Bewertung setzt zu dem Zeitpunkt ein, als sie anfängt ihre Jugend mit dem Leben zu vergleichen, das deutsche jugendliche Mädchen heute führen können. Dies führt zu einem nur schwer aufrechtzuerhaltenden Vergleich, da sie als Mutter dreier Jugendlicher, die im Deutschland des 21. Jahrhunderts aufwachsen, ihre Jugend mit der ihrer Kinder vergleicht. Aus diesem Blickwinkel gesehen ergeben sich für Jugendliche in Deutschland heute gewisse Freiheiten, die zum Zeitpunkt von Saras Jugend auch in deutschen Familien nicht die Norm dargestellt haben mussten. Mit diesem Vergleich schneidet die Bewertung ihrer eigenen Jugend schlechter $a b$ als wenn sie wenn sie ihre Jugend mit der deutscher Frauen ihres Alters vergleichen würde. Dennoch wäre auch in diesem Fall die Bandbreite der Freiheiten und Entscheidungsmöglichkeiten die einzelnen Frauen in Deutschland damals hatten, größer.

\subsubsection{Unzureichende medizinische Versorgung in Somalia}

Erst nachdem ich nochmals nach ihrer Kindheit frage, erzählt Sara ein wenig aus diesem Lebensabschnitt.

S: „Ich habe sechs Geschwister gehabt, aber (--) an vier kann ich mich nicht mehr erinnern, weil sie, äh, sie sind als Babys gestorben. Und, eines, an das letzte, ein Junge, an den kann ich mich schon noch ein bisschen erinnern. (---) Der ist Anfang der 80er Jahre gestorben. Aber ich habe auch noch einen älteren Bruder." (I 1, 51-54)

Sara berichtet, dass sie insgesamt sieben Kinder waren und nur drei (Sara und zwei ältere Brüder) die Kindheit in Somalia überlebt haben. Da ihre Geschwister aber schon als Säuglinge verstorben seien, könne sie sich an vier der Babys nicht mehr erinnern.

Während sie vom Tod ihrer Geschwister berichtet, gerät sie immer wieder ins Stocken. Auch wenn diese Erlebnisse lange Zeit zurück liegen und Sara teilweise keine Erinnerung mehr an ihre Brüder hat, könnte eine Lesart der stockenden Erzählung sein, dass es Sara schwer fällt über diese Zeit zu sprechen. Vermutlich hat sie den Einstieg in das Interview bewusst mit ihrer Jugend gewählt, um nicht mit den emotional belastenden Ereignissen ihrer Kindheit beginnen zu müssen. Als ich nachfrage, woran die Babys gestorben seien, zeigt sich Sara verwundert, so als höre sie diese Frage zum ersten Mal. 
S: „Naja, halt Fieber oder Gelbsucht, solche Krankheiten halt, man weiß es auch nicht so genau in Somalia, da hat man nicht so viele Ärzte wie in Deutschland, kein so ein Gesundheitssystem. Man wird krank und die Leute, die halt nicht die Möglichkeit haben oder kein Geld haben, da musst du zusehen wie deine Kinder sterben oder wenn du Hilfe bekommst, dann hast du Glück gehabt. Dann hast du Glück gehabt.“ (I 1, 56-60)

Sie berichtet daraufhin von Krankheiten, die möglicherweise der Grund für den Tod ihrer Geschwister gewesen sein könnten, betont aber gleichzeitig, dass man das in Somalia nicht genau sagen könne. Das liege daran, dass es nicht so viele Mediziner_innen gäbe und auch kein mit Deutschland vergleichbares Gesundheitssystem. Das habe zur Folge, dass der Zugang zu medizinischer Hilfe aufgrund des Ärztemangels schwierig und teuer sei. Nur wenige Menschen können sich eine Behandlung leisten - medizinische Hilfe ist laut Sara für die Mehrzahl der Menschen in Somalia Glückssache. Sind Kinder betroffen, muss im schlimmsten Fall zugesehen werden wie die eigenen Kinder sterben.

Auch an dieser Stelle schildert Sara ihre Geschichte im ständigen Vergleich mit dem Leben in Deutschland. Durch diese Brille des Vergleichs zeigt sich besonders deutlich wie unvermittelt der Tod über somalische Familien hereinbrechen kann. Ob Fieber oder sonstige Krankheiten, es ist immer wahrscheinlich, dass der Krankheitszustand nicht überlebt wird, weil es keine medizinische Hilfe gibt oder man sich diese schlicht finanziell nicht leisten kann. Aus ihrer heutigen Sicht weiß Sara, dass in anderen Ländern wie Deutschland ein Gesundheitssystem existiert, das im Krankheitsfall genutzt werden kann - nicht wie in ihrem Heimatland. Sie und ihre Familie haben früh erlebt, wie hilflos Babys Krankheiten ausgeliefert sind und wie schnell Fieber zum Todesurteil werden kann. Auch an dieser Stelle gerät Sara ins Stocken, erzählt zögerlich, was den Eindruck verstärkt, dass es ihr schwer fällt, über die frühen Verluste ihrer Geschwister zu sprechen. Trotzdem aber berichtet sie davon, vielleicht um mir als deutscher Zuhörerin zu verdeutlichen, wie sehr sich das Leben in Somalia vom Leben in Deutschland unterscheidet.

Sara fährt fort, als sie jedoch erzählt, dass sie ihre Mutter seit 20 Jahren nicht mehr gesehen hat, beginnt sie zu weinen und wir unterbrechen das Interview. Da sie nach der Pause zu einem anderen Thema wechselt, erfahre ich nichts von ihrem Vater, weiß daher nicht, ob dieser noch lebt, in Somalia geblieben ist oder ob sie noch Kontakt haben. 


\subsubsection{Sozialer Druck und der Wunsch nach Anpassung}

Wir nehmen das Interview wieder auf und sprechen über weiblicher Genitalbeschneidung in Somalia.

S: „Normalerweise in Somalia, für die Mädchen ist das Kultur. Das muss man machen [...] Es ist ganz normal. Unter Mädchen sprechen wir schon drüber, aber die Mädchen, die nicht beschnitten sind, sind sozusagen schlechte Mädchen. Das hat für uns was mit Reinheit zu tun, es ist unsere Kultur. (--) Jedes Mädchen macht das.“(I 1, 93-98)

Sara spricht aus zwei Perspektiven: einmal ganz allgemein von „man“, dann wieder aus Sicht der Mädchen, einer Gruppe, der sie sich offensichtlich zugehörig fühlt, da sie in diesem Moment von „wir“ spricht. Saras Aussage „das muss man machen“ erweckt den Eindruck, dass es sich um eine Gepflogenheit handelt, etwas, das unhinterfragt besteht. Spricht Sara aus ihrer Sicht als Mädchen, ist die Beschneidung Teil ihrer ,Kultur', und alle die, die nicht beschnitten sind, würden als „schlechte“ und „unreine“ Mädchen beschimpft. Aus der Perspektive des Mädchens, das Sara zum Zeitpunkt ihrer eigenen Beschneidung war, verdeutlicht Saras Schilderung, wie groß das Bedürfnis für sie und die anderen Mädchen gewesen sein muss, selbst beschnitten zu werden, um nicht als „schlecht" und „unrein“ beschimpft zu werden. Aus dieser Schilderung kann gelesen werden, dass es für die Mädchen ein positiv besetztes Ereignis handeln muss, eines, das ihnen hilft ihre Unreinheit abzulegen und ein ,gutes' Mädchen zu werden.

So wird weibliche Genitalbeschneidung schon in der Kindheit als ,Tradition' konstruiert, mit Attributen wie ,rein' und ,gut' positiv belegt und als bedeutender Teil somalischer ,Kultur' an die Mädchen weitergegeben. Gleichzeitig wird den Mädchen vermittelt, Trägerinnen dieser Kultur und für deren Weitergabe an die nächste Generation verantwortlich zu sein. Saras Schilderungen zeigen, wie die Mädchen dies bereits verinnerlicht haben und untereinander dafür sorgen, dass der Wunsch der ,Tradition' ${ }^{\text {zu entsprechen }}$ an die unbeschnittenen Mädchen weitergegeben wird, indem sie als ,schlechte" Mädchen ausgegrenzt werden. So wird weibliche Beschneidung zu einer positiven Ereignis, das Zugehörigkeit und Zusammenhalt sichert.

Im Folgenden beschreibt Sara wie groß sie den Druck der Mädchen verspürt hat, warum sie diesem am Ende nicht standhalten konnte und sich beschneiden ließ, obwohl ihre Mutter eine Beschneidungsgegnerin war.

S: „Bei mir war meine Mutter dagegen. Sie hat gesagt: „mach das nicht!“, aber ich wollte es. Mich haben die anderen Mädchen unter Druck gesetzt, beleidigt und dann musste ich es machen. Oder, nee, freiwillig. Aber meine Mutter hat gesagt „Ohne mich!“ Ich bin dann von M. weggegangen in ein anderes Dorf, das zehn Stunden mit dem Auto entfernt war. In ein Dorf, in dem meine Großeltern gewohnt haben. Ich bin da mit meiner Tante hin, das habe ich freiwillig gemacht.“ (I 1, 102-107) 
Sara, damals zehn Jahre alt, nimmt eine lange Reise auf sich, um am Ende so zu sein wie alle anderen auch: beschnitten und ,rein'. Aus ihrer damaligen Position gab es keine andere Alternative als den Weg zu gehen, den alle Mädchen gehen. Ihr Wunsch, wieder zur Gruppe der, guten' Mädchen zu gehören, war dominanter als die Warnung ihrer Mutter. Aus heutiger Sicht zeigt sich Sara bezüglich dieser Entscheidung ambivalent: Einerseits erinnert sie sich an den Druck der Mädchen und ihrem Wunsch, so zu sein wie sie und wieder zur Gruppe zu gehören. Auf der anderen Seite ist da aber auch ihre Mutter, die ihr abgeraten hatte, auf die sie aber nicht gehört hat. Sara schwankt bis zum Ende des Interviews in der Bewertung, ob es Druck oder doch eine freiwillige Entscheidung war. Je nachdem welche Position Sara einnimmt, verändert sich die Bewertung der damaligen Situation. Aus heutiger Perspektive rückt Saras Bedürfnis nach Zugehörigkeit und Anerkennung in ihrer Peergroup in den Hintergrund, und sie spricht von ihrer eigenen, freiwilligen Entscheidung. Der Druck der Mädchen spielt aus dieser Perspektive keine Rolle mehr, dann nennt Sara es eine „freiwillige“ Entscheidung und verurteilt sich selbst dafür, nicht auf die Mutter gehört zu haben. Aus dieser Perspektive beschreibt sie sich als ungehorsames und unreifes Kind:

S: „Weil meine Mutter das schon verstanden hatte. Bei der Geburt hatte sie viele Probleme, man hat immer extra schneiden müssen und sie hat viel geblutet und so. Und sie hat dann gesagt „Mach das nicht, das ist schlecht!“ [...] Das hat sie schon verstanden. Und sie hat gesagt, dass sie diese Probleme erlebt hat und sie deshalb nicht möchte, dass ich dasselbe erlebe. Deshalb hat sie gesagt: „Lass es lieber!“. Ich habe aber nicht zugehört, ich war halt unreif, ein Kind! Mit zehn Jahren, da hat man noch keinen Kopf." (I 1, 120 128)

Aus Saras Erzählung sind Enttäuschung und Selbstvorwürfe zu lesen. Sie ist von sich selbst enttäuscht, dem Druck der Freundinnen nicht standgehalten zu haben und wirft sich vor, nicht auf ihre Mutter gehört zu haben. In diesem Moment zeichnet sie ein Bild von sich als schwaches Kind, das nicht zugehört hat. Aus dieser Sicht betrachtet bleibt für Sara heute nur zurück, damals eine falsche Entscheidung getroffen zu haben, die sie heute offensichtlich bereut. Während des gesamten Interviews kämpft Sara mit sich beziehungsweise mit dem zehnjährigen Kind, das sich für die Beschneidung entschieden hat, und der erwachsenen Frau von heute, die diese Entscheidung als Fehlentscheidung bewertet. Dass sich das zehnjährige Mädchen von damals aber auch gegen ihre erwachsene Mutter durchgesetzt hat, ist für Sara kein Zeichen ihrer Stärke, sondern eines von Unreife.

Auch am Ende des Interviews kommt Sara noch einmal auf ihre eigene Beschneidung zu sprechen, auf den Tag an dem sie es nicht geschafft hat ,nein' zu sagen. Noch einmal wird deutlich, wie sehr sie unter ihrer Entscheidung leidet. Aber in diesem Teil stellt sie sich die Frage, weshalb ihre Mutter ihre Beschneidung nicht verhindert hat: 
S: „Und mit diesen Schmerzen und all den Problemen, kann man nicht weitergeben, deinen Kindern geben. Du musst doch deine Kinder ein bisschen schützen. Meine Mutter hat versucht mich zu beschützen, aber sie hätte (-) ich bin schon ein bisschen traurig, sie hätte von Anfang an „,nein“ sagen sollen. „Du bist mein Kind und ich sage, du machst das nicht!“ Aber sie war halt zu lasch - sie hat mich einfach gehen gelassen." (I 1, 425-429)

Jetzt sieht Sara den Tag aus Sicht ihrer Mutter, vielleicht auch aus ihrer heutigen Position als Mutter einer Tochter. Und aus dieser Perspektive spricht auch Enttäuschung, allerdings ist sie an dieser Stelle von ihrer Mutter enttäuscht. Aus Sicht der erwachsenen Sara, hätte sich ihre Mutter mehr für Sara einsetzen, sie zurück halten müssen. Stattdessen, so nennt es Sara, habe sie sie „einfach gehen lassen“, obwohl sie genau wusste, was auf Sara zukommen und in welchem Ausmaß dieser Eingriff sie künftig beeinträchtigen würde.

S: ,[ja] trotzdem hätte sie sagen müssen „Scheiß auf den Druck! Du bist mein Kind und wenn ich sage du machst das nicht, dann machst du das nicht!" Sie hätte einfach stärker sein sollen, nicht mich einfach frei lassen und zu meiner Tante lassen. Aber das ist halt Schicksal, vielleicht hat Gott gewollt, dass ich das erlebe." (I 1, 431-434)

Sara ist der Meinung, ihre Mutter hätte vehementer auftreten müssen, spürbarer dafür kämpfen, dass Sara nicht beschnitten wird. Sara geht noch einmal auf den Druck ein, den die Mutter hätte ignorieren sollen, doch spielt dieser sowohl in ihrer Erzählung aus Sicht des Mädchens als auch jetzt aus Sicht einer erwachsenen Frau eine Rolle. In machen Momenten entsteht der Eindruck, sie würde diesen heute noch spüren und wüsste wie schwer es ist, sich diesem Druck zu entziehen. Dann aber, so wie in eben diesem letzten Abschnitt, blickt sie aus ihrer heutigen Perspektive auf die damalige Entscheidung und scheint nicht zu verstehen, weshalb es zu dieser kommen konnte.

Sara ist hin- und her gerissen, scheint nicht zu wissen, bei wem sie letztlich die Enttäuschung über die Entscheidung für Beschneidung lassen soll. Mal hat ihre Mutter Schuld, dann wieder sie, das zehnjährige Mädchen von damals. In seltenen Momenten spricht Sara auch von höheren Umstände, dann wird ihre Beschneidung zu ihrem Schicksal oder einer gottgewollten Sache $\mathrm{zu}$ einem Ereignis, für das niemand die Verantwortung trägt: weder Sara noch ihre Mutter. Sie wählt damit eine Strategie, die ihr nur kurz ein Entkommen bietet. Am Ende sucht sie doch wieder die Schuld bei dem Mädchen, das sie damals war oder ihrer Mutter, die sie nicht ausreichend beschützt hat. Es macht den Eindruck, als könne Sara diesem Kreislauf im Kopf nicht entkommen. 


\subsubsection{Vom Tag der Beschneidung}

Ein weiterer Faktor, der aus Saras Geschichte deutlich wird, bezieht sich auf die Unwissenheit, die im Hinblick auf Genitalbeschneidung besteht. Zwar erzählt Sara, dass ihre Mutter verstanden hat, dass es sich um eine Praktik handelt, die Schwierigkeiten mit sich bringt, doch sagt Sara, dass sie ihr das nie erklärt habe. So wusste Sara bis zum Tag ihrer Beschneidung nicht, was auf sie zukommen würde. Laut Sara betrifft diese Unwissenheit sowohl diejenigen, die beschnitten werden, als auch diejenigen, die den Eingriff durchführen. Bei Sara handelte es sich dabei um eine Dorffrau, die davon lebte, Mädchen zu beschneiden. Sara nennt sie „Hebamme“, sagt aber nicht, ob diese Frau eine entsprechende medizinische Ausbildung hatte, woraufhin ich noch einmal nachfrage:

I: „Und sie hat aber schon so ein bisschen Vorkenntnisse gehabt und hat schon gewusst, was sie da macht?"“

S: ,Sie hat schon mehrere Mädchen gehabt, so am Tag hat sie vielleicht 20 Mädchen gemacht.“ (I 1, 139-141)

Da ich als Interviewerin nicht präzise nach einer medizinischen Ausbildung oder medizinischen Vorkenntnisse frage, versteht Sara meine Frage in Bezug auf Erfahrungen, die die Beschneiderin im Beschneiden anderer Mädchen hat. Aus dieser Sichtweise hatte die Frau Vorkenntnisse: Wer am Tag 20 Mädchen beschneidet, wird schon wissen was zu machen ist, so Saras Interpretation. Fraglich bleibt aber, ob diese Frau anatomische Kenntnisse mitgebracht hat und wusste welche Körperteile sie abschneidet und welche medizinischen Folgen ihr Eingriff hat oder später mit sich bringen kann. Laut Sara verhält es sich auf Seiten der Mädchen ähnlich, auch sie seien unwissend, wären eher neugierig, was wohl passieren würde. Auch Sara berichtet neugierig auf ihren Beschneidungstag gewesen zu sein, den sie so unwissend wie die restlichen Mädchen auch begangen habe.

S: „Ich wäre fast gestorben, weißt du? Fünf Frauen saßen auf mir, die Luft haben sie mir abgedrückt, das Ganze ohne Narkose, das war schrecklich! Sie drücken dich bis zur Bewusstlosigkeit. (-) und auf einmal lassen sie dich doch los, eine Minute und wieder (---), immer wieder." (I 1, 451-454)

Besonders überrascht zeigt sich Sara über die Brutalität der Frauen. Sie spricht von Todesängsten, die sie während des Eingriffs hatte, weil sie so viele Frauen niedergedrückt und ihr die Luft abgedrückt haben. Sara erzählt diesen Teil nicht als Gesamtes, sie streut während des Interviews immer wieder kurze Sequenzen dieses Tages ein. Als Zuhörerin ist es so, als würden in Saras Kopf immer wieder einzelne Bilder dieses Tages auftauchen, die sie plötzlich loswerden muss, häufig ohne viele Emotionen zu benennen oder zu zeigen. Erst am Ende, in diesem eben zitierten Teil, zeigt sie ihre Gefühle, ihr Entsetzen über die Art und Weise des Umgangs mit ihr als zehnjährigem Mädchen. Sara erzählt diesen Teil laut und eindringlich. Nach dem Nieder- 
drücken, Festhalten und Luft abdrücken, folgt die Beschneidung ,ganz ohne Narkose“. Wie viele Schmerzen mit dem Wegschneiden von Genitalien verbunden sind, sagt sie nicht - vermutlich fehlen ihr dafür die Worte. Eine Lesart der Betonung der fehlenden Narkose könnte aber sein, dass Sara deutlich machen wollte mit wie viel Schmerzen Beschneidung verbunden ist und dass es in Somalia üblich ist diese ohne Narkose zu ertragen. Mithilfe des Hinweises auf die fehlende Narkose, versucht Sara mir ein Bild von dem Leiden zu verschaffen, das sie in diesem Moment durchlebt hat, von der sie der Meinung ist, dass auch ich, ohne Mädchenbeschneidung erlebt zu haben, verstehe, was da mit ihr als Mädchen passiert ist.

Wenn Sara von ihrer eigenen Beschneidung berichtet, zeigt sich an vielen Stellen, wie sehr sie unter den psychischen wie physischen Konsequenzen leidet. Das Erleben derartiger Brutalität und ihrer Hilflosigkeit, die Schmerzen und Folgen lassen Sara bis heute nicht los. Sie bereut es sich für die Beschneidung entschieden zu haben. Sie betont aber, dass sie erst in Deutschland das Ausmaß des Eingriffs begriffen habe.

S: „Als ich noch in Somalia war, habe ich gedacht, das ist normal, weil sonst heiratet dich niemand, dann bist du eine schlechte Frau oder so.“ (I 1, 495-497).

Ohne den kulturellen Rahmen verliert die ,Tradition` für Sara den Sinn. Die Begründungen in Verbindung mit dem Druck, waren in Somalia ausschlaggebend für ihre Entscheidung für Beschneidung, doch existieren diese in Deutschland nicht länger. In Deutschland erfährt Sara das Gegenteil: sich für ihre ,Tradition' rechtfertigen zu müssen. In den Jahren in Deutschland wird Sara bewusst,

S: „dass es auch andere Möglichkeiten gibt. Die Mädchen hier sind frei, wie meine Tochter halt, sie darf sein wie Gott sie geschaffen hat. Warum macht man denn das? Ich habe festgestellt, dass ich viele Nachteile von der Beschneidung habe. Deswegen habe ich meine Meinung geändert. (I 1, 497500)

In Somalia war die Beschneidung eine Norm, der Sara und die anderen Mädchen entsprechen wollten. Der Druck ihrer Peergroup, den Sara vor allem durch Beschimpfungen und sozialen Ausschluss erlebte, nennt Sara „enorm“. Hinzu kommt die Unwissenheit, die die Mädchen neugierig macht und wie Sara dazu antreibt, zum Fortbestand der Praktik beizutragen.

\subsubsection{Saras Erfahrungen in Deutschland}

Als Sara nach Deutschland kommt ist sie siebzehn Jahre alt. Sie berichtet, dass sie von ihrer Mutter von Somalia bis nach Ägypten begleitet wurde und von dort aus alleine nach Deutschland geflogen sei, wo sie von ihrem bis dahin unbekannten Ehemann in Empfang genommen wurde. Ihre Mutter 
habe sie danach nie mehr gesehen. Sie landete in einem fremden Land und wurde von einem ihr unbekannten Mann abgeholt. Sara selbst beschreibt diesen Anfangszustand folgendermaßen:

S: ,[...] ich war 17. Ich war in einem neuen Land, mein Kopf war überfordert. Alles war für mich durcheinander." (I 1, 181-182)

Noch bevor Sara die Möglichkeit hat ein wenig Ordnung in dieses Durcheinander zu bringen, wird sie nach nur einem Monat in Deutschland schwanger:

S: „Da war ich völlig durcheinander. Und mein erstes Kind habe ich verloren. Die ganze Umstellung, der Stress, im vierten Monat" (I 1, 184-185).

Sara erzählt ohne Umschweife von diesem Erlebnis. Sie nutzt dieses als Einstieg in ihre Schilderungen zum Leben als beschnittene Frau in Deutschland. Mit dieser ersten Schwangerschaft schlägt ihr Ehemann Sara vor, die Schwangerschaft gynäkologisch begleiten zu lassen. Er selbst begleitet sie zu der Untersuchung. Sara beschreibt bereits diesen Vorschlag als befremdlich, da sie bis dahin noch nie von Gynäkolog_innen gehört hatte. Sie habe sich trotz dieser Verwunderung jedoch auf den Vorschlag ihres Mannes eingelassen und sei zum Gynäkologen gegangen.

S: Genau. Ich wollte mich am Anfang auch nicht ausziehen! In Somalia gibt es keinen Frauenarzt! Das war das allererste Mal in meinem Leben, das ein Arzt, also ein Mann gesagt hat, dass ich mich ausziehen soll! (lächelt) Das ist schlimm! [...]Ja. Dann hat es zwei Stunden gedauert bis ich mich getraut habe. Mein Mann hat mich ein bisschen ermutigt und hat gesagt, spätestens bei Geburt muss ein Arzt kommen oder eine Ärztin um das Kind rauszuholen. Es spielt doch überhaupt keine Rolle, ob es ein Mann oder eine Frau ist, hat er gesagt. Ein Arzt ist ein Arzt und er hilft dir. Versuch es. Beim ersten Mal habe ich es nicht geschafft, aber beim zweiten Mal habe ich mich getraut.“ (I 1, 169-177).

Sara ist an dieser Stelle aufgebracht, erhebt die Stimme beim Erzählen und wiederholt „ein Arzt, also ein Mann“, so als wollte sie sichergehen, dass ich gehört habe, dass es sich tatsächlich um einen männlicher Arzt handelte, der von ihr verlangte sich auszuziehen. Obwohl diese Geschichte lange Zeit zurück liegt, wird in diesem Moment des Erzählens noch deutlich, dass die damalige Situation, sich von einem fremden Mann gynäkologisch untersuchen zu lassen, Sara sehr beschämte. Es gelingt ihr beim ersten Besuch in der gynäkologischen Praxis nicht ihre Scham zu überwinden, trotz des guten Zuredens ihres Mannes. Offen bleibt, warum Saras Ehemann nicht bereits von Anfang an eine weibliche Gynäkologin aufgesucht hatte, das habe ich Sara nicht gefragt.

Als Sara schließlich ihre Scham überwindet, zeigt sich der Arzt schockiert:

S: „Arzt war schockiert. Sehr schockiert. Er hat gesagt „Wo soll das Kind raus kommen?“, dann hat er gesagt „sie braucht extra ( ), da muss man 
schneiden. Um GOTtes Willen!“ Er war halt wie die Deutschen, geschockt!““ (lächelt) (I 1, 164-166)

Sara erzählt von dem schockierten Verhalten des Arztes, es scheint aber mehr als würde sie ihn im Rückblick dafür belächeln - damals vermutlich nicht, aber heute wo Sara die Reaktion von „den Deutschen“ wie sie sagt, kennt, schmunzelt sie ein wenig darüber. Deutlich wird, dass im Augenblick der Untersuchungssituation zwei Vorstellungen aufeinander getroffen sind: Saras Vorstellung von ,normalen“ Genitalien und die des Gynäkologen.

Nachdem Sara von ihrer ersten gynäkologischen Untersuchung berichtet hat, beginnt ein langer Weg auf der Suche nach einem geeigneten Gynäkologen. Im Anschluss an diese erste Schwangerschaft und die erlittene Fehlgeburt berichtet sie von weiteren Komplikationen, die sich aus eben dieser ersten Schwangerschaft ergeben haben. Bei jedem Arztbesuch, ob bei niedergelassenen Gynäkolog_innen oder in Kliniken, sei sie immer wieder auf unwissende Ärzt_innen gestoßen. Nach vielen entwürdigenden und beschämenden Erfahrungen mit deutschen Gynäkolog_innen trifft Sara auf einen Arzt zu dem sie Vertrauen aufbauen kann.

S: „Der erste Arzt war schockiert. Dem habe ich das dann halt erklärt, dass es unsere somalische Kultur ist. Er hat dann gesagt „Da ist ja schrecklich! Ihr armen Menschen" hat er gesagt. Ich erinnere mich noch genau. Und dann war er ganz vorsichtig, er hat immer wieder gesagt: „Es passt schon, ich tu Ihnen nicht weh, wir machen das ganz langsam!“ [...] Aber dann habe ich es geschafft, Gott sei Dank. Das war nicht so schwierig und er hat es auch ganz vorsichtig gemacht." (I 1, 208-213)

Sara berichtet, dass auch dieser Arzt schockiert gewesen sei, der Unterschied jedoch gewesen sei, dass er sich für Saras Geschichte und den Hintergrund der Mädchenbeschneidung interessierte habe. Sie habe ihm ihre somalische ,Kultur' erklären können. Außerdem berichtet sie, dass er vorsichtig mit ihr umgegangen sei und ihr die Untersuchungsschritte erklärt habe. Eine Lesart dieser Aussage könnte sein, dass die Gynäkolog_innen vorher weniger vorsichtig mit ihr umgegangen sind und ihr weniger erklärt haben. Möglich ist jedoch auch, dass die zugewandte Art, die Sara an diesem Gynäkologen zu schätzen scheint, dazu führt, dass sich Sara behutsamer begleitet fühlt. Laut Sara hat ihr die Tatsache, dass der Gynäkologe ihre Erklärung zur Praktik anhörte, vorsichtig mit ihr umgegangen ist und ihr die einzelnen Untersuchungsschritte erklärt hat, dabei geholfen, sich auf die gynäkologische Untersuchung einzulassen. Sie äußert sich erleichtert („Gott sei Dank“) darüber, am Ende auf einen Gynäkologen getroffen zu sein, der einen adäquaten Umgang mit ihr als beschnittener Frau gefunden hat. Das von Interesse und Empathie geprägte Verhalten des Arztes Sara gegenüber ermöglichte eine Untersuchung, von der Sara am Ende sagt: „Das war gar nicht so schwierig.“ 


\subsubsection{Vom Prozess des Umdenkens}

Nachdem Sara von ihren Erfahrungen mit deutschen Gynäkolog innen berichtet und deutlich gemacht hat, wie sie sich den medizinischen Umgang mit beschnittenen Frauen wünscht, berichtet sie im Folgenden wie schwierig es erst einmal für sie selbst war, sich von ihrer Normvorstellung des weiblichen Genitales zu trennen. So schockierend wie es für deutsche Gynäkolog_innen gewesen sein muss, erstmals infibulierte Genitalien zu sehen, so schockierend nimmt Sara die unbeschnittenen Genitalien ihrer Tochter wahr:

S: „Für mich war es auch schockierend als meine Tochter geboren ist, weil ich kenn nur meine Scheide und ich habe meiner Tochter ihre gesehen, so wie Gott sie geschaffen hat. Und ich habe gedacht, ich habe ein behindertes Kind! Ich war schockiert! Ich habe eine Gänsehaut bekommen, Herzklopfen, was ist passiert? Ich habe das dann meinem Mann erzählt und er hat nur gesagt: „Das ist genau wie Gott es erschaffen hat, du brauchst dir keine Sorgen um deine Tochter zu machen! Deine Tochter ist nicht behindert! Alles ist in Ordnung." Er hat mich getröstet, aber ich habe gedacht, es ist was passiert." (I 1, 249-256)

Sara spricht von Schock, weil die Genitalien ihrer Tochter aus ihrer Sicht „behindert" waren. Sie berichtet davon, sich Sorgen gemacht zu haben, die auch nicht weniger wurden als ihr Ehemann ihr versicherte, dass die Genitalien ihrer Tochter ,in Ordnung“ seien. Offensichtlich fällt es Sara sehr viel schwerer als ihrem Mann, sich von ihrer Vorstellung eines ,normalen' Genitales zu lösen. Sara selbst ist seit ihrem zehnten Lebensjahr infibuliert, möglicherweise erinnert sie sich nicht an den Zustand ihrer unbeschnittenen Genitalien. Für sie stellt die Infibulationsnarbe die Norm dar. Sara verkörpert die ,Tradition“ weiblicher Beschneidung und identifiziert sich damit. Ihre Erzählung verdeutlicht, wie schwer es ihr fällt, sich von ihrem Bild zu lösen. Zumal hinter dem Bild unbeschnittener Genitalien in Genitalbeschneidung praktizierenden Bevölkerungsgruppen Zuschreibungen wie ,krank', ,triebhaft ${ }^{\star}$ oder ,unrein' stehen, die Sara internalisiert zu haben scheint und die beim Anblick der Genitalien ihrer Tochter in ihr hochkommen. In diesem Zusammenhang erwähnt Sara, dass es somalische Familien gibt, die sich von dem Bild behandlungsbedürftiger Genitalien nicht lösen können und Wege finden, ihre Töchter trotz des Verbotes in Deutschland, beschneiden lassen.

S: „Ich erkläre auch unseren Frauen, also denen aus unserer Heimat, „Machst du das?“. Viele sagen „NATÜRLICH, ich habe es erlebt, das muss sie auch erleben!“. Dann sage ich „Das ist Dummheit! Das darfst du nicht! Hier in Deutschland kommst du in den Knast! “ Aber viele Frauen, die fliegen in die Heimat und wenn sie zurückkommen, dann sind die Kinder schon beschnitten. Keiner untersucht, keiner kontrolliert!“ (I 1, 229-233)

Sara berichtet von Frauen, die ihre Töchter beschneiden lassen wollen, weil die Mädchen Gleiches erleben sollen wie ihre Mütter. Neben den Zu- 
schreibungen, die mit beschnittenen Genitalien einhergehen, erzählt Sara auch, dass der Eingriff von manchen Frauen als wichtige Erfahrung betrachtet wird, die ihre Töchter, genau wie sie selbst, durchmachen sollen. Sara nennt die dahinter stehende Begründung nicht. Aus meiner Sicht könnte es daran liegen, dass Genitalbeschneidung in vielen Bevölkerungsgruppen als Initiationsritual gilt, das den Übergang vom Mädchen zur Frau kennzeichnet und Mädchen nicht zu Frauen werden können, ohne das zu durchleben. Diesen Schritt im Leben eines Mädchen auszulassen, würde dann bedeuten, keine ,richtige' Frau in die Gesellschaft zu entlassen - so könnte eine Begründung dafür lauten, dass Mädchen Beschneidung aus Sicht ihrer Mütter durchleben müssen. Wie schwierig es ist, Menschen zu erreichen, die von der Ansicht überzeugt sind, genitale Beschneidung sei ein nicht auszulassender Schritt im Leben eines Mädchens, bestätigt auch Saras Ehemann, mit dem ich im Anschluss über Beschneidung spreche. Er sagt: ,[...] derjenige, der nicht reif ist, dich zu verstehen, mit dem kannst du nicht diskutieren."

Sara aber gelangt in Gesprächen mit ihrem Ehemann zu der Überzeugung, ihre Tochter unbeschnitten zu lassen, und ist heute davon überzeugt, die richtige Entscheidung getroffen zu haben. Heute spricht Sara von „Dummheit", wenn sie hört, dass andere somalische Frauen ihre Töchter beschneiden lassen wollen. Auch wenn Sara am Beispiel ihrer Tochter noch einmal deutlicher sieht, wie sehr sie selbst durch ihre Beschneidung eingeschränkt wurde:

S: „Aber das ist halt, also auch für mich selber, persönlich kann ich das jetzt beurteilen, weil ich kenne diese Zeit wie meine Tochter, weil wir sind jetzt zwei verschiedene Menschen. Aber es könnte sein, wenn ich so wäre, dann hätte ich bessere Gefühle.“ (I 1, 272-275)

Sara vergleicht ihr Leben mit dem ihrer unbeschnittenen Tochter und ist der Meinung, dass sie beide „zwei verschiedene Menschen“ sind. Aus Saras Sicht wäre sie heute ein anderer Mensch, wäre sie damals unbeschnitten geblieben - ein Mensch wie ihre Tochter, die laut Sara „bessere Gefühle“ habe. Was Sara damit meint, bleibt offen. Sara äußert sich vorsichtig, vielleicht, weil sie nicht die passenden Worte findet, um zu beschreiben, welcher Art diese Gefühle sind. Sie spürt aber, dass ihre unbeschnittene Tochter „,bessere Gefühle“ hat.

\subsubsection{Saras Perspektive auf die Aufklärungs- und Beratungsarbeit in Deutschland}

Nachdem wir lange über ihre Beschneidung, ihre Erfahrungen in Deutschland und ihre Tochter gesprochen haben, frage ich Sara, ob sie denkt, dass Aufklärungsarbeit gegen Genitalbeschneidung in Deutschland und die Begleitung betroffener Frauen sinnvoll ist. Ich frage sie auch, wie Angebote gestaltet sein müssten, um am Ende von Betroffenen angenommen zu wer- 
den. Sara ist der Meinung, dass grundsätzlich Bedarf an Aufklärung für betroffene Frauen besteht, da viele nicht um die Konsequenzen von Mädchenbeschneidung wüssten. Um diese überwinden zu können, schlägt Sara vor, somalischen Frauen mehr Aufklärung in ihrer Muttersprache zu kommen zu lassen:

S: „Dass man eine somalische Frau holt, die die Infos weiterleitet. Das hat auch Esra (eine somalische Frau, die bereits seit vielen Jahren Beratungen für somalische Frauen durchführt) mir gesagt, die hat das vor so zu machen. Das ist auch eine gute Idee, dass man sagt, wenn ein Mädchen, ein Junge ist ok, aber das man überhaupt das nicht macht. Und wer es trotzdem macht, der kommt 20 Jahre in Knast. Das ist gut.“ (I 1, 304-307)

Sara wünscht sich mehr Aufklärungsarbeit gegen weibliche Genitalbeschneidung, betont gleichzeitig, dass die Beschneidung von Jungen für sie in Ordnung sei.

S: „Ja, so mit somalischen Büchern, wenn es das gegeben hätte, wäre ich hin. Weil wenn die hierher kommen, können sie die Sprache nicht, da wäre es mit somalischen Büchern oder wenn da eine somalische Frau da wäre, die übersetzt. Wie Esra z.B., die eine Beratungsstelle hat, das wäre schön. Aber ob die kommen würden, das weiß ich nicht (---).“(I 1, 401-404)

Aufklärung gegen Genitalbeschneidung und die Begleitung betroffener Frauen sollte in der Muttersprache stattfinden. Wenn Bücher und Informationsmaterialien auf Somali wären, wäre das aus Saras Sicht hilfreich, dann könnten die Frauen die Informationen gegebenenfalls noch einmal nachlesen. Sara denkt dabei an all die Frauen, die über weniger gute Deutschkenntnisse verfügen und auf Übersetzung angewiesen sind. Da sie selbst somalische Frauen zu Beratungsstellen und sonstigen Terminen begleitet, weiß sie aus Erfahrung, dass die Sprache eine große Herausforderung für somalische Frauen ist. Gäbe es eine solche Beratungsstelle, würde Sara diese aufsuchen, weil sie glaubt, dass sie ihr und den anderen somalischen Frauen Unterstützung bieten könnte. Und trotz der Begeisterung, die sie persönlich für dieser Idee hat, ist sie sich nicht sicher, ob die anderen somalischen Frauen eine solche Beratungsstelle nutzen würden. Als ich dann sage, dass es bereits Beratungsstellen gibt, die beschnittene Frauen beraten, die Frauen aber nur selten kommen, sagt Sara, dass es wichtig sei, Genitalbeschneidung in andere Themenfelder einzubetten.

S: „Genau, nicht direkt Beschneidung sondern allgemein. Schwangerschaft, Eheprobleme, Erziehung, alles was es in Deutschland halt auch gibt. Jugendamt, Hilfeplan das und das. Dass man halt so Dinge erklärt, Infos gibt, dann kommen die. Und dann irgendwann gibt man ihnen Infos zu Beschneidung in die Hand, dann können sie selber, freiwillig das nehmen.[...] Genau. Weil sie denken „Lieber nicht sprechen, lieber schweigen!“ Und viele die das Problem erlebt haben, die machen so weiter. Das ist auch nicht ok. Man muss das doch selber sehen, was man erlebt hat!" (I 1, 416-424) 
Die Einbettung von Genitalbeschneidung in breitere Beratungsfelder betrachtet Sara für sinnvoll und effektiv. In erster Linie gelte es, das Vertrauen der Menschen zu gewinnen. Erst dann wäre ein Gespräch zu einem heiklen Thema wie Genitalbeschneidung möglich. Gäbe es zudem noch Informationsbroschüren auf Somali, könnten sich Betroffene diese einfach mitnehmen - erst einmal ohne über die Thematik sprechen zu müssen. Betroffenen Frauen wäre es auf diese Weise möglich, nachzulesen was genau ihnen widerfahren ist und darüber nachzudenken, ob beziehungsweise welche Art von Unterstützung sie benötigen. Sara betont die Wichtigkeit von Freiwilligkeit, dass betroffenen Frauen die Wahl gelassen werden sollte, ob und wo sie sich Unterstützung holen. Die Aussagen Saras zeigen auch, dass ein Beratungsangebot nicht zwingend für alle Frauen passend sein muss. Einige würden, so Sara, nicht gerne von ihren Erfahrungen sprechen, weil sie gelernt hätten, über diese Erfahrung zu schweigen. Um diesen Frauen aber auch Informationen zu den Folgen und dem in Deutschland bestehenden Verbot von Genitalbeschneidung zukommen zu lassen, wären Informationsbroschüren in somalischer Sprache geeignet.

Eines ist Sara besonders wichtig, das betont sie während des Interviews zwei Mal: Wurden Familien in Bezug auf die Folgen weiblicher Genitalbeschneidung und die gesetzliche Lage in Deutschland aufgeklärt und entscheiden sich trotzdem ihre Töchter beschneiden zu lassen, dann müssen diese strafrechtlich verfolgt und belangt werden. Sie fordert für die Eltern ein hohes Strafmaß, was noch einmal zeigt, wie sehr sie selbst Genitalbeschneidung ablehnt. Sie hat kein Verständnis für Menschen, die in Deutschland leben und sich trotz der Gesetzeslage für die Beschneidung ihrer Töchter entscheiden. Zudem fordert sie neben der Bestrafung der Eltern, die ihre Tochter beschneiden lassen auch strengere Kontrollen. Sie ist der Meinung, dass Töchter nach Heimaturlauben untersucht werden sollten, etwas, das bis heute nicht stattfinden würde. Sara sagt dies ziemlich aufgebracht, weil sie einfach nicht verstehen kann, weshalb dies nicht regelmäßig kontrolliert und geahndet wird.

\subsubsection{Saras Blick auf die Rolle der Männer im Kontext weiblicher Genitalbeschneidung}

Da Sara der Meinung ist, dass Männer maßgeblich zum Erhalt der Praktik beitragen, beschränkt sie die Notwendigkeit von Aufklärungsarbeit nicht allein auf Frauen.

S: „Genau, ja. Weil die denken „Ich muss der erste sein!“ Das kommt von Seiten der Männer denke ich. Ich diskutiere zwar auch mit meinem Mann, er sagt „Ich bin nicht so“, aber ich glaube das aber. „Warum?" sage ich dann. Das ist Kultur, sagt man? Als Mädchen muss man von oben bis unten zu sein 
bevor sie ein Mann sieht. Das muss sie halt beweisen, dass er erster ist. (lächelt) Und das kommt von den Männern denke ich. Das ist meine Meinung. Aber viele Männer sagen „Nein, das ist nicht unsere Meinung!“““ (I 1, 332337)

Aus Saras Blickwinkel sind Männer für Genitalbeschneidung verantwortlich, was daran liege, dass Männer jungfräuliche Frauen heiraten wollten, um, wie Sara es nennt, „der erste“ sein zu können. Sara spricht aber auch vom Argument der ,Kultur', dem sich Männer bedienen würden, um Genitalbeschneidung zu rechtfertigen. Obwohl Sara von Gesprächen mit Männern berichtet, die dieser Argumentation nicht folgen und Beschneidung ablehnen, glaubt sie daran, dass sie diejenigen sind, die weibliche Beschneidung fordern. Dass Sara eine solche Meinung vertritt, scheint paradox, da es in ihrem Fall ihre Freundinnen waren, die sie zu Beschneidung gedrängt haben, es war ihre Tante, die sie begleitet hat und eine Beschneiderin, die sie beschnitten hat. Ihr Ehemann dagegen lehnt Genitalbeschneidung ab, sorgt dafür, dass Sara im Umgang mit ihrer eigenen Beschneidung Unterstützung bekommt und setzt sich dafür ein, dass ihre gemeinsame Tochter nicht beschnitten wird. Sara scheint trotz ihrer persönlichen Erfahrungen der Meinung zu sein, dass Männer Genitalbeschneidung vorantreiben - das denkt sie unabhängig davon, was Männer ihr diesbezüglich sagen. Im Hinblick auf die wiederkehrende Schuldfrage, die in Saras Interview eine bedeutende Rolle spielt, könnte die Zuweisung der Schuld an ,die Männer' auch als Ausweg aus der sie sehr belastenden Schuldfrage gelesen werden. Wenn , die Männer' an weiblicher Genitalbeschneidung Schuld sind, sind sie und ihre Mutter entlastet.

Sara fährt fort und begründet ihre Meinung mit dem mangelnden Vertrauen, das Männer in Frauen hätten. Aus Saras Sicht hätten sie Angst davor, von ihren Ehefrauen hintergangen zu werden und ihre Macht über sie zu verlieren. Für diese Denkweise wäre, so Sara, das Frauenbild in den Köpfen der Männer verantwortlich:

S: „Und dass halt diese Vorverurteilung weggelassen wird. Und egal wie, dass das Mädchen geheiratet wird, egal ob beschnitten oder nicht. Dass sie sie halt so akzeptieren wie Gott die Frau erschaffen hat. Und nicht sagen „Ja, die ist offen und die hat vielleicht andere Männer gehabt" so wie vielleicht die europäischen Frauen, die vielleicht Erfahrungen sammeln oder auch nicht, wer weiß, nur Gott weiß es. Und auch die heiraten und der Mann nimmt sie so wie sie ist! Egal ob sie vor ihm fünf Männer gehabt hat oder 30. Das interessiert ihn nicht! Hauptsache er liebt sie, er nimmt sie so wie sie ist, egal ob sie dick oder dünn oder dumm ist. So müssen unsere Männer auch denken! Nicht dieses Denken „Du musst beschnitten sein“, das ist Dummheit!“( (I 1, 346-354)

Sara ist der Meinung, dass Männer von unbeschnittenen Frauen denken, dass diese sexuelle Erfahrungen gesammelt haben könnten, bevor sie in die Ehe gehen. Vorehelichen Geschlechtsverkehr würden Männer mit europäi- 
schen Frauen verbinden, nicht aber somalischen Frauen zugestehen, so Saras Sicht auf die männliche Meinung. Ob die Frauen tatsächlich sexuelle Erfahrungen gesammelt hätten oder nicht, das wisse dann nur Gott. Eine fehlende Beschneidung würde bedeuten, dass es für die Männer nicht mehr nachvollziehbar wäre, ob Frauen vor der Ehe sexuellen Verkehr gehabt hätten oder nicht, so beschreibt Sara die Situation, und das würde Männer verunsichern und dazu führen, dass sie nur beschnittene Frauen wollten. Sara selbst ist der Meinung, dass Frauen ihre Sexualität leben dürfen wie es ihnen gefällt Männer hätten darüber nicht zu urteilen. Sie plädiert für mehr Toleranz, weniger Vorverurteilung und fordert ein Umdenken von den Männern. Sara wünscht sich eine Entwicklung hin zu Männern, die Frauen heiraten, weil sie sie lieben - unabhängig davon ob oder wie viel sexuelle Erfahrung die Frau vor der Ehe gesammelt hat. An dieser Stelle erzählt Sara aufgebracht, nahezu verärgert. Sie scheint nicht zu verstehen, weshalb sich eine solche Denkweise halten kann. Auch hier vergleicht sie ihr damaliges Leben in Somalia mit dem Leben, das sie heute in Deutschland führt. Sie sagt, dass die „europäischen Frauen" selbst entscheiden dürften, ob sie sexuelle Erfahrungen sammeln oder nicht, das würde Männer nicht interessieren, weil sie ihre Frauen aus Liebe heiraten würden. So wünscht es sich auch Sara, dann würden aus ihrer Sicht auch unbeschnittene Frauen akzeptiert. Doch glaubt Sara nicht daran, dass sich dies bald verändern wird:

S: „Das dauert noch sehr lange, denke ich! (--) Erstmal muss man Männer aufklären und fragen, warum macht ihr das? Warum wollt ihr das? Viele werden sagen „Das kommt vom Sudan, von der arabischen Kultur. Das ist nicht die richtige somalische Kultur, es ist irgendwie rüber nach Somalia gekommen". Aber warum macht man das? Im Sudan macht man das bis heute, habe ich gehört. Erwachsene Frauen sogar erleben das, ob das stimmt weiß ich nicht. Aber eine Frau hat mir erzählt, wenn ihr Mann nach Europa reist oder egal, halt irgendwo in der Welt reist, lässt er vorher seine Frau wieder zunähen. Er geht arbeiten drei Jahre, vier Jahre oder egal, manchmal auch nur ein Jahr, je nachdem wie er Geld hat, fliegt er nach Sudan zurück und macht sie wieder auf. Was ist das?" (I 1, 356-364)

Sara erklärt, dass Männer an weiblicher Beschneidung festhalten würden, obwohl sie nur vage Antworten auf die Frage hätten, woher die Praktik käme. Sie spielt die Frage in einem fiktiven Dialog zwischen ihr und einem von Genitalbeschneidung überzeugten Mann durch und greift dabei vermutlich auf die Antworten zurück, die sie von manchen Männern in Diskussionen bekommen hat. Für Sara handelt es sich bei dem durchgespielten Dialog um keine befriedigende Antwort, lediglich ein Versuch etwas zu rechtfertigen, das sich je nach Perspektive bewährt hat: Es sei irgendwie Teil der somalischen ,Kultur' geworden, keiner könne aber genau sagen, woher die Praktik stamme. Als Sara fortfährt und von der Geschichte über den Umgang des sudanesischen Mannes mit seiner Ehefrau berichtet ist sie wütend und fragt 
laut: „Was ist das?“. Sara ärgert sich darüber, dass dieser sudanesische Mann seine Frau als Besitz begreift und über ihre Sexualität bestimmen darf - egal von wo auf der Welt. „Wie ein eigenes Zimmer, das er zusperrt und wieder aufsperrt wie er will“, so sieht das Sara.

Letztlich zeigen Saras Erläuterungen, dass wohl noch einige Männer, die sie kennt, weibliche Beschneidung mit bestimmten Zuschreibungen verbinden, die sie als Begründung für den Erhalt der Praktik nennen. Eine wichtige Zuschreibung scheint die der Jungfräulichkeit und Treue zu sein, die beschnittene Frauen verkörpern. Sie beschreibt die Angst der Männer, die Kontrolle darüber zu verlieren, ihre eigene Ehefrau könne vor der Ehe bereist sexuelle Erfahrungen gesammelt haben oder während der Ehe untreu sein. Aus Saras Sicht dient weibliche Genitalbeschneidung somit der Kontrolle und gibt Männern Sicherheit. Da Sara jedoch den gesellschaftlichen Kontext verlassen hat, bewertet sie Genitalbeschneidung aus heutiger Sicht nicht nur als „Dummheit“", sie ärgert sich auch sehr, wenn sie auf Männer trifft, welche die Praktik verteidigen, insbesondere über jene, die wie Sara selbst in Deutschland leben. Sie wünscht sich, dass unbeschnittene Frauen akzeptiert werden. Um diesem Ziel näher zu kommen, müssen sich laut Sara nicht nur die Männer verändern, auch Frauen müssen „nein“ sagen lernen und kämpfen.

S: „Und die Frauen müssen halt „nein“ sagen lernen und die brauchen bisschen eine Erklärung, dass es auch ein anderes LEBEN gibt." (I 1, 374375)

Für Sara sind am Ende nicht allein , die Männer' Schuld am Erhalt der Praktik, auch Frauen tragen dazu bei, weil sie sich nicht gegen Beschneidung wehren würden. Das sei aus ihrer Sicht jedoch nicht ohne „Erklärung“ möglich. Vermutlich spielt sie an dieser Stelle auf ihren eigenen Umdenkprozess an und erinnert sich daran, dass dieser wachsen musste und mit vielen Gesprächen verbunden war. Daher weiß Sara aber auch, dass es wichtig ist, dass beide - Mann und Frau - verstanden haben, dass weibliche Genitalbeschneidung keine Vorteile bringt und unbeschnittene Genitalien nicht behandlungsbedürftig sind. Nur dann ist es möglich, Mädchen vor dem Eingriff zu schützen.

\subsubsection{Zusammenfassung}

Sara erzählt ihre Geschichte durch die Brille des Vergleichs: Sie betrachtet ihr Herkunftsland, dessen Gepflogenheiten und ihr Großwerden im gesellschaftlichen Kontext Somalias im ständigen Vergleich zu ihrem heutigen Leben in Deutschland und dem Leben ihrer Kinder, die im 21. Jahrhundert in Deutschland aufwachsen. Dies führt an vielen Stellen zu einem Ungleichgewicht, da das Leben, das Sara damals in ihrer Kindheit in Somalia verbracht 
hat, nicht mit dem heutigen Leben ihrer eigenen Kinder in Deutschland zu vergleichen ist. In diesem Vergleich findet die Zeit, die seit Saras Kindheit und Jugend vergangen ist - sowohl in Somalia als auch in Deutschland keine Berücksichtigung. Aus diesem Vergleich heraus zeichnet Sara ein Bild, das Deutschland einseitig positiv und Somalia einseitig negativ darstellt. Für Sara selbst ergibt sich daraus großes Bedauern und Trauer darüber, nicht wie ihre eigenen Kinder mit den heutigen Freiheiten groß geworden zu sein. Sie habe keine Wahlmöglichkeiten gehabt, habe machen müssen, was ihre Eltern ihr gesagt haben.

Ähnlich verhält sich Sara, wenn sie von ihrer eigenen Beschneidung berichtet. Mit dem Wissen und der Erfahrung einer beschnittenen Frau, die heute um die negativen Konsequenzen, des Eingriffes weiß, blickt sie auf ihre Beschneidung zurück. Aus dieser Perspektive heraus stellt sich Sara wiederkehrend die Schuldfrage: Hat sie Schuld, weil sie dem Druck ihrer Freundinnen nicht standhalten konnte, oder ihre Mutter, die sie vor dem Eingriff stärker schützen hätte sollen, oder sind es ,die Männer', die an der ,Tradition “ festhalten? Sara hinterlässt den Eindruck, diesem Kreislauf nicht entkommen zu können,. Sie blickt immer wieder zurück auf sich selbst als zehnjähriges Mädchen und gelangt zu der Einschätzung, selbst daran Schuld zu sein, heute mit beschnittenen Genitalien leben zu müssen, da sie nicht auf ihre Mutter gehört und sich „freiwillig“ dem Eingriff unterzogen hat. Aus Saras Interview ist zu entnehmen, dass sie den Eingriff erst nach dem Verlassen ihres Heimatlandes in Frage stellt. Während Beschneidung in Somalia ,normal' war, erfährt Sara, dass sie in Deutschland für Entsetzen sorgt und sie sich für ihre beschnittenen Genitalien und ihre damalige Entscheidung für den Eingriff rechtfertigen muss. Letztlich bringt sie ihre Migration in ein Genitalbeschneidung ablehnendes Land dazu, ihre ,Tradition' zu hinterfragen und zu erfahren, dass damit etliche negative Konsequenzen verbunden sind. Diese Informationen, aber auch die Tatsache, dass ihr Ehemann weibliche Genitalbeschneidung ablehnt, sorgen dafür, dass Sara ihre eigene Tochter unbeschnitten lässt. Gleichzeitig aber bleibt Sara allein mit der Frage zurück, warum sie selbst den Eingriff erleben musste und wem hierfür Schuld zuzuschreiben ist.

In Bezug auf die Arbeit gegen weibliche Genitalbeschneidung in Deutschland vertritt sie die Meinung, dass Familien über die Konsequenzen des Eingriffs und die Gesetzeslage in Deutschland aufgeklärt werden müssen. Die Aufklärungsarbeit oder Informationsbroschüren sollten aus Saras Sicht auf Somali sein, um den Menschen die Chance zu geben, die Informationen auch vollständig zu verstehen. Sara betont außerdem die Notwendigkeit, auch Männer über weibliche Genitalbeschneidung aufzuklären, da sie aus ihrer Sicht maßgeblich am Erhalt der ,Tradition“ beteiligt seien. Aus Saras Interview geht ferner hervor, dass weibliche Genitalbeschneidung nicht mithilfe eines Informationsgesprächs beendet werden kann. Vielmehr handelt es sich 
aus ihrer Sicht um einen Prozess, der wie bei Sara selbst, mit wiederkehrenden Gesprächen zu den Folgen des Eingriffs verbunden ist. Die Abkehr von einer gesellschaftlich etablierten Norm ist eine große Herausforderung, die, wie in Saras Interview deutlich wird, Zeit braucht.

\subsection{Nadifa}

Nadifa lebt seit 18 Jahren in der Schweiz und stammt ursprünglich aus Zentralsomalia. Sie ist Mutter einer siebenjährigen Tochter und alleinerziehend. Der Kontakt zu Nadifa erfolgte über eine gemeinsame Freundin. Noch vor dem Treffen nahm ich telefonisch Kontakt mit ihr auf. Bereits in diesem Telefonat kam Nadifas Ärger über den Umgang mit beschnittenen Frauen in der Schweiz zur Geltung, der sich durch das gesamte Interview zieht und dem sie schon in diesem ersten Telefonkontakt Luft macht. Nadifa ist besonders verärgert über den von ihr als herabwürdigend empfundenen Umgang mit Frauen somalischer Herkunft. Da dies bereits vor dem eigentlichen Interview gesagt wurde, gestaltet sich der Einstieg in das Interview mit Nadifa dahingehend, sie direkt auf ihre bereits geäußerte Kritik anzusprechen. Die Erzählungen zu Nadifas Leben in Somalia geraten deshalb in den Hintergrund, während ihrem Ärger viel Raum gelassen wird. Hinzu kommt, dass meine Fragen an manchen Stellen ihren Ärger verständnisvoll aufgreifen und dazu führen, dass dieser immer wieder Thema wird. Nadifa ist zum Zeitpunkt des Interviews Anfang vierzig.

\subsubsection{Nadifas Erfahrungen in der Schweiz}

Nadifa kommt direkt auf ihre Erfahrungen im schweizerischen Gesundheitssystem zu sprechen, die sie ohne Umschweife wie folgt beschreibt:

$\mathrm{N}$ : „Also Beschneidung, eigentlich (---) ich wusste nicht, was die Beschneidung hier für die Europäer bedeutet, bis ich hierher kam und in einer Klinik war. Also, da haben sie mich untersucht, da waren sie weiß ich nicht was, so schockiert und dann kamen viele MÄNNER, also viele ÄRZTe, also wirklich ein ganzes ÄrzteTEAM und sagten „Oh, was haben Sie?! Was ist das?!“ Also wirklich eine, also eine Szene, die ich nicht erwartet habe und äh, wenn ich heute zurück schaue, denke ich, es war nicht gut, was sie mit mir gemacht haben." (I 2, 14-19).

Nadifa steigt ohne Umschweife mit Erzählungen zu ihren persönlichen Erfahrungen in der Schweiz ein. Sie berichtet erst einmal nichts von ihrem Leben und der Rolle der Beschneidung in Somalia. Stattdessen spricht sie von „den Europäern“, für die Beschneidung eine große Bedeutung hat. Sie 
erzählt von der Reaktion der Ärzte auf ihr beschnittenes Genitale und von „,vielen Männern“, die sich um sie scharten, um ihre Genitale zu betrachten. Nach dieser Begutachtung sei das männliche Ärzteteam schockiert gewesen und hätte sich nicht erklären können, was mit Nadifas Genitale passiert sein könne. Damals habe Nadifa diese Reaktion irritiert, heute denkt sie, dass diese Reaktion nicht angebracht war. Ähnlich wie Sara wurde auch Nadifa von schockierten Ärzten umringt, die sich mit Entsetzen fragten, was wohl mit ihrem Genitale passiert sei. Nadifa zeigt sich darüber allerdings sehr aufgebracht, was besonders im Rückblick auf die vielen Männer, die ihr Genitale betrachteten, deutlich wird. Trotz ihres Ärgers bleibt sie mit der Bewertung der Situation vorsichtig, äußert sich nahezu zweifelnd: „Wenn ich heute zurück schaue, denke ich, es war nicht gut, was sie mit mir gemacht haben."In Anbetracht des sehr entwürdigenden und voyeuristischen Umgangs der Ärzte mit dieser Frau, die schon im ersten Telefonkontakt deutlich ausspricht, dass sie mit dem Umgang mit Beschnittenen in der Schweiz nicht zufrieden ist, handelt es sich um eine vorsichtige Bewertung ihrer Erfahrung mit schweizerischen Ärzten. Im weiteren Verlauf wird jedoch deutlich, dass sich Nadifa weniger über die schockierten Ärzte und die Tatsache, zur Schau gestellt worden zu sein, ärgert, als vielmehr von der Überheblichkeit mit der „die Europäern“ oder „die Schweizer“ aus ihrer Sicht mit Somalier_innen umgehen - unabhängig davon, ob diese noch in Somalia leben oder in die Schweiz immigriert sind. Nadifa variiert in der Wahl der Bezeichnungen, mal spricht sie von „den Europäern“, mal von „den Schweizern“, für sie scheinen beide Begriffe dieselbe Bedeutung zu haben.

$\mathrm{N}$ : ,Also ich als Somalierin habe KEINE großen Probleme mit der Beschneidung also so als SOLCHE. HAUPTSAche ist, dass die Leute aufgeklärt werden, aber nicht das es wirklich zu so einer Druckausübung kommt. Also „Ihr sollt das nicht machen!“, „Das ist verboten“ und das ist so und es ist nicht äh menschenrechtlich, das ist nicht (-) die Menschen müssen verstehen, WARum sollten sie damit aufhören? (---) Also, die Leute sollen auch das Gefühl, dass es ihnen selbst überlassen ist, ob sie das weiter möchten oder nicht." (I 2, 22-28)

Nadifa verbindet mit „den Europäern“ oder „den Schweizern“ Bevormundung, da sie den Somalis vorschreiben wollen, was diese zu tun beziehungsweise zu lassen hätten. Nadifa selbst zählt sich zur Gruppe der Somalis, der Gruppe der immigrierten Somalis. Sie betont, selbst keine Probleme mit weiblicher Beschneidung zu haben, solange „die Leute“ aufgeklärt seien, dürften sie aus Nadifas Sicht selbst entscheiden, ob sie ihre Töchter beschneiden lassen. Aus Nadifas Sicht seien es ,die Europäer", die aus Beschneidung ein Problem machen. Mithilfe von Gesetzen und Verboten würden laut Nadifa Somalis unter Druck gesetzt, anstatt ihnen zu erklären warum Beschneidung unvorteilhaft sei. Am Ende, betont Nadifa, seien es allein „die Leute", die entscheiden könnten, ob sie ihre Töchter beschneiden ließen oder 
nicht - das sollten „die Europäer“ akzeptieren. Nadifa spricht an dieser Stelle von ,den Leuten“, was als Abgrenzung zu denjenigen Menschen, die sich für weibliche Genitalbeschneidung aussprechen, gelesen werden könnte. Nadifa schafft mithilfe dieser Begriffswahl eine Distanz, weil sie selbst nicht zu der Gruppe gehört, die ihre Tochter beschneiden lässt, aber eben auch nicht zu der Gruppe, die anderen vorschreibt, wie sie sich zu verhalten haben. Die Stelle zeigt deutlich, dass Nadifa das bevormundende Verhalten „der Europäer“ ablehnt. Sie nimmt die Arbeit gegen Beschneidung als „europäischen“ Imperativ wahr, dem sich Somalis zu beugen hätten. Die Tatsache, dass Mädchenbeschneidung physische und psychische Folgen haben kann, spielt für Nadifa in diesem ersten Teil des Interviews eine untergeordnete Rolle.

Erst nachdem sie ihrem Ärger über das bevormundende Verhalten „der Europäer" Luft machen konnte, äußert sie sich kritisch zu Mädchenbeschneidung, auch wenn es erst einmal nur ein kleines Zugeständnis an die aus ihrer Sicht vorwiegend „europäischen“ Gegner_innen der Mädchenbeschneidung ist.

$\mathrm{N}$ : „Und äh (---) für mich ist die Beschneidung, diese Art der Beschneidung, diese totale Beschneidung, also dass wirklich große Teil der Organe weg geschnitten werden, also und dieses Zusammennähen und all das finde ich (---) NIcht in Ordnung. Für mich ist das nicht in Ordnung.“ (I 2, 32-35)

Nadifa scheint es schwer zu fallen, zuzugeben, dass sie Infibulation ablehnt. Sie korrigiert sich vier Mal, um sicher zu gehen, dass sie nicht missverstanden wird und am Ende deutlich wird, dass sie nur die Beschneidung ablehnt, bei der ein großer Teil der Organe weg geschnitten und zusammengenäht wird. Erst nachdem Nadifa verdeutlich hat, dass sie wirklich nur diese Form meint, ist es ihr möglich einzuräumen, dass dieser Eingriff auch für sie als Somaliern nicht in Ordnung ist. Ohne dass Nadifa noch einmal explizit auf ,die Europäer“ und „die Somalier“ eingeht, könnte eine Lesart ihrer Darstellung von Mädchenbeschneidung sein, sich keinesfalls auf die Seite „der Europäer" stellen zu wollen und deshalb zu betonen, dass nur diese eine Form der Mädchenbeschneidung nicht in Ordnung sei. Es scheint Nadifa ein großes Anliegen zu sein sich deutlich von „den Europäern“" abzugrenzen, weil sie dieser Gruppe Überheblichkeit und Bevormundung im Umgang mit ihr und ihren Landsleuten vorwirft.

\subsubsection{Eltern haben die Entscheidungsmacht}

Nachfolgend berichtet Nadifa von der Rolle, die Beschneidung für Eltern spielt, und dass es niemandem zustehe, Eltern in der Entscheidung für oder gegen Beschneidung einzuschränken, da alle Eltern nur das Beste für ihre Töchter wollten. 
N: „Und IHRE Rolle [die der Eltern, Anmerkung I.I.] ist GRÖßER als derjenige, der einfach sagt, dass sei nicht gut. Schlussendlich es ist seine Tochter, es ihr Leben was gerettet wird oder ähm, besser gestaltet wird. Und sie soll einfach diese Freiheit haben, diese Wahl haben „Ok, ich will das oder ich will das nicht". Und man muss die Leute einfach respektieren, man muss einfach diesen TON wählen, mit welchem TON man mit den Leuten redet, man muss nicht von unten äh, von oben herab schauen und sagen „ICH weiß, was für dich besser geeignet ist, weil du bist ein hilfloser Nomade oder jemand, der wirklich keine Chancen im Leben hat! MACHST du das mal lieber nicht!", weil dann kommt überhaupt kein Resultat. Dann ist das eine BlockADE, dann OHNE dass man weiß, woran es wirklich ähm wie sagt man, SCHEITERT, kommt eine Scheiterung. Es wird nichts mehr passieren. (-) man muss einfach Leute, äh, wirklich vielleicht vorher trainieren wie sie mit den Leuten umgehen (--)“(I 2, 80-90).

Aus Nadifas Sicht wollen Eltern grundsätzlich das Beste für ihre Kinder, so sei das auch, wenn sich Eltern für weibliche Beschneidung entscheiden würden. Aus Sicht dieser Eltern sei Genitalbeschneidung das Beste für ihre Töchter und aus diesem Grund würden sie sich für den Eingriff entscheiden, eine Entscheidung, die den Eltern aus Nadifas Sicht zustehe. Eltern nun einfach zu verbieten, ihre Töchter beschneiden zu lassen, sei kontraproduktiv, da ein solches Verbot als Geste der Bevormundung und Entmündigung interpretiert würde, was in letzter Konsequenz dazu führe, dass Eltern ihre Töchter erst Recht beschneiden ließen. Stattdessen betont Nadifa die Notwendigkeit eines respektvollen Umgangs mit den Eltern. Für sie bedeutet das in erster Linie, die Menschen nicht ,von oben herab“ zu behandeln, ihnen auf Augenhöhe zu begegnen und sie nicht auf eine untergeordnete Ebene zu stellen. Somalis auf die Ebene des ,hilflosen Nomaden“ zu stellen sei unangemessen, das würden die Menschen spüren und, wie Nadifa es nennt, zu einer „Blockade" führen, womit sie meint, dass ein solcher Umgang zu keiner Veränderung bei den Menschen führe. Bevor über weibliche Beschneidung gesprochen würde, müsse in einem ersten Schritt trainiert werden wie mit ,den Leuten" umzugehen sei, so Nadifa. In diesem Teil des Interviews zeigt sich wieder deutlich, dass sich Nadifa über die Art und Weise des Umgangs mit ihr und ihren Landsleuten ärgert. Aus Nadifas Sicht handelt es sich nicht um einen Kontakt auf Augenhöhe, vielmehr würden die Gespräche „von oben herab" stattfinden. Es sei kein Austausch, von dem beide Seiten profitieren könnten. Vielmehr würde den Menschen von Anfang an vermittelt, dass sie unmündig seien und deshalb gesagt bekommen müssten, was gut für sie sei. Nadifa nimmt den Umgang „,er Europäer“ mit Somalis sowohl in Somalia selbst, als auch in der Schweiz als überheblich und übergriffig wahr, was dazu führe, dass Aufklärungsarbeit gegen Beschneidung scheitere. Sie selbst ist verärgert über diesen Umgang - ein Ärger, der das Interview dominiert. Es scheint, als nutze Nadifa mich und das Interview als Sprachrohr um in die 
,europäische‘ Welt zu tragen, wie sehr sie die Überheblichkeit und Bevormundung durch_Europäer_innen' ärgert.

Auch im nachfolgenden Abschnitt betont Nadifa noch einmal, dass die Art und Weise in der Aufklärungsarbeit geschieht, entscheidend ist:

$\mathrm{N}$ : „Aber wie packt man das an? Das ist das Problem. Weil wir haben auch in anderen Bereichen gesehen, dass die Europäer oder die westlichen Länder einfach so kommen und einfach so herumkommandieren (lächelt) und so, dass ist gut, das ist nicht gut. Also mittlerweile haben die Leute einfach die Nase voll von denen, diesem Herumkommandieren. Also wir sind quasi GAST in unserem eigenen Land, in vielen Bereichen." (I 2, 101-105)

Laut Nadifa habe die Geschichte gezeigt, dass „Europäer“ oder „westliche Länder" nur herum kommandieren würden, den Menschen in ihrer eigenen Heimat das Gefühl gäben, zu Gast zu sein. Von diesem Verhalten hätten „die Leute" die Nase voll, sie wollten stattdessen selbst bestimmen, was gut beziehungsweise schlecht für sie ist. Nadifa spricht an dieser Stelle des Interviews nicht mehr allein von weiblicher Genitalbeschneidung. Sie benennt die Folgen des Kolonialismus, die sich aus ihrer Sicht noch immer darin äußern, dass „Europäer“ sich über sie als Somalierin stellen. Eine Folge, die in Nadifa auslöst, „die Schweizer“ oder „die Europäer“ abzulehnen, ihre Bevormundung nicht länger ertragen zu können. Letztendlich kann die starke Abgrenzung Nadifas von „den Schweizern“ auch als Schutzmechanismus gelesen werden. Vermutlich hat Nadifa selbst einen solchen Umgang erlebt und sie versucht, sich durch die Abgrenzung zu „den Schweizern“ oder „den Europäern" vor erneuten Herabwürdigungen zu schützen. Eine weitere Lesart ist, dass Nadifa diese Strategie zwar schützt, ihr aber gleichzeitig im Weg steht, da sie nicht zugeben kann, dass weibliche Genitalbeschneidung negative Folgen mit sich bringt. Würde sie grundsätzlich einräumen, Genitalbeschneidung abzulehnen, wäre sie der Meinung ,der Europäer“, was sie zu vermeiden versucht, da sie die Grenze zwischen ihr als Somalierin und „den Europäern", die sie mit Entmündigung und Bevormundung verbindet, aufrechterhalten will.

\subsubsection{Eine Frage der Haltung}

Nachdem sich Nadifa deutlich vom Umgang „der Schweizer“ oder „,der Europäer" mit weiblicher Genitalbeschneidung distanziert hat, ist ihr möglich einzuräumen, dass Aufklärungsarbeit zwar sinnvoll ist, aber bestimmten Regeln zu folgen hat.

N: „Äh, und um überhaupt das zu bekämpfen, müssen Frauen involviert sein, also Frauen müssen einfach dagegen kämpfen. Aber nicht kämpfen in dem sie sagen „Das ist verboten!“”, „Das ist schlecht!“, „Macht das nicht!“, 
sondern einfach den Leuten erklären WARUM sollte das nicht gut sein? (--) Wozu sollte das gut sein, dass man das nicht mehr macht?" (I2, 51-55)

Nadifa spricht sich für die Einbindung von Frauen in die Arbeit gegen Beschneidung aus, die jedoch keinesfalls mithilfe von Verboten oder Bevormundungen arbeiten dürften. Stattdessen fordert Nadifa, dass den Menschen erklärt wird, warum es sich um eine schädliche Praktik handelt. Noch einmal betont sie an dieser Stelle, wie sie sich den Dialog zum Thema weibliche Beschneidung mit somalischen Familien wünscht. Dann erst benennt sie den Eingriff als Problem:

N: „Es ist nicht so ein einfaches Problem, viele Mädchen sterben dadurch, viele Mädchen haben sehr viele Probleme SPÄTER damit, also es, ich glaube nicht, dass es SOO ein kleines Problem sein sollte. Ist es nicht.“ (I 2, 98-101)

An dieser Stelle spricht Nadifa die Infibulation als ,gravierendes Problem“ an, das viele negative Folgen für die Mädchen mit sich bringen kann. Es scheint, als habe Nadifa den ersten Teil des Interviews benötigt, um ihrer Wut über das „europäische“ Verhalten Somalis gegenüber Luft zumachen, und sie könne sich jetzt ehrlich zum eigentlichen Thema äußern. Jetzt darf sie aussprechen, dass Infibulation „sehr viele Probleme“ mit sich bringt, mehr noch „,viele Mädchen dadurch sterben“, ohne dass ich als Zuhörerin denken könnte, sie wäre gleicher Meinung wie „die Europäer“. An dieser Stelle zeigt sich, dass Nadifa die Gründe, die gegen Beschneidung sprechen, sieht und teilt. Für Nadifa steht fest, dass weibliche Genitalbeschneidung erst abgeschafft werden kann, wenn den Menschen durch Erklärungen die Möglichkeit gegeben wird, die Gründe nachvollziehen zu können.

Als wir uns weiblicher Beschneidung unter somalischen Migrantinnen in der Schweiz zu wenden, äußert sich Nadifa ähnlich deutlich wie auch schon im ersten Teil des Interviews:

$\mathrm{N}$ : „Ja. Also, die die ähm Leute, die in diesem Bereich etwas tun, die sind von OBen herab auf die Leute „Macht das nicht, ihr macht euch STRAFbar!“‘. Und äh (---) ich verstehe überhaupt nicht die ganze, ganze (---) Kampagne, also die ganze, ganze äh, ja ÜBERHAUPT äh, die ganze POLItik gegenüber Migrantinnen hier, dass sie NICHT Beschneidung weiterführen, ich verstehe es NICHT als Solches. Weil ERSTENS: es ist nicht vorhanden, die Beschneidung findet nicht statt! Zweitens es ist nicht da, wo es wirklich gebraucht wird. Es ist in Somalia, wo die Leute wirklich Hilfe brauchen und (---) Ratschläge, sie brauchen Leute, die sich für sie einsetzen“ (I 2, 146-152)

Ähnlich wie in Somalia würden ,die Europäer", in diesem Fall „die Schweizer“, somalischen Migrant_innen „,von oben herab“ begegnen. Von Nadifas Blickwinkel aus betrachtet existiert in der Arbeit mit Migrant_innen kein Umgang auf Augenhöhe, frei von Belehrungen oder Drohungen. Sie beschränkt die Äußerung nicht auf Aufklärungsarbeit gegen Mädchenbeschneidung, sie lehnt alle Angebote, die sich an Migrant_innen richten, ab. 
Grund der Ablehnung ist immer wieder die bevormundende Art des Kontaktes.

Hinzu kommt, dass Nadifa vor allem die „Kampagnen“ gegen weibliche Beschneidung nicht verstehen kann, da sie der Meinung ist, dass Mädchenbeschneidungen in der Schweiz nicht durchgeführt würden. Diese finden laut Nadifa in Somalia statt, nicht aber in der Schweiz. Aus diesem Grund seien alle Kampagnen und Bemühungen der Schweizer aus ihrer Sicht sinnlos. Stattdessen müsse mehr Aufklärungsarbeit in Somalia stattfinden, dort müsse sich für die Menschen eingesetzt werden. Aus dieser Stelle kann gelesen werden, dass Nadifa unterstreichen möchte, dass zu wenig mit somalischen Migrant_innen gesprochen wird, sonst wüssten „die Schweizer“, dass Genitalbeschn̄eidung unter somalischen Migrant_innen in der Schweiz nicht stattfindet. Vermutlich möchte Nadifa mit ihrer Aussage zeigen, dass nicht nur die Aufklärungsarbeit gegen Genitalbeschneidung herablassend und bevormundend ist, sondern die gesamte Migrationspolitik und die daraus resultierenden „Kampagnen“. Diese gingen an den Bedürfnissen der Menschen vorbei, weil kein gleichberechtigtes Gespräch zwischen Migrant_innen und Vertretern der schweizerischen Mehrheitsgesellschaft stattfinden würde. Nadifa, die mit somalischen Migrant_innen im Kontakt zu sein scheint, sieht jedenfalls keinen Grund für „Kampagnen“ gegen weibliche Genitalbeschneidung in der Schweiz. Nachfolgend erzählt Nadifa von ihren somalischen Freunden und erklärt, weshalb diese ihre Töchter nicht beschneiden lassen.

N: „Äh, also die Somalier, ähh, ich habe Kontakt zu Somalierinnen, aber mit denen ich Kontakt habe, das sind auch die, die besser AUSgebildet sind, Frauen, die hier ihr Leben so MEIstern, äh und das sind Frauen, die ÜBERHAUPT nicht äh, also die Beschneidung spielt überhaupt keine Rolle in ihrem Leben. Sie haben Kinder und für sie ist Beschneidung ein Thema, das abgeschlossen ist. Mit solchen Frauen habe ich Kontakt, aber die anderen Frauen, die immer noch an die Sache glauben und vielleicht irgendwann eine Chance suchen, mit solchen habe ich keinen direkten Kontakt.“ (I 2, 216222)

Nadifa erzählt, dass ihre freundschaftlichen Kontakte sich auf Somalierinnen beschränken, die Mädchenbeschneidung mittlerweile ablehnen. Zu Frauen, die noch an der Notwendigkeit von Beschneidung festhalten und nach Möglichkeiten suchen, ihre Töchter beschneiden zu lassen, hat Nadifa keinen direkten Kontakt. Was Nadifa mit „direkt" meint, bleibt unklar. Vermutlich bekommt sie über ihre Freundinnen mit, dass es Somalierinnen gibt, die an Mädchenbeschneidung festhalten, ist mit diesen Frauen aber nicht befreundet. Nadifa schränkt mit der Aussage in diesem Abschnitt ihre vorherige Aussage, Mädchenbeschneidungen würden unter somalischen Migrant_innen in der Schweiz nicht gemacht, deutlich ein. Sie hat nur Kontakt zu Frauen, die Mädchenbeschneidung ablehnen, mit denjenigen, die, wie sie sagt, „,immer noch an die Sache glauben“, ist Nadifa nur nicht befreundet. Somit kann 
keine Aussage zum Verhalten der Frauen getroffen werden, die an der Notwendigkeit von Mädchenbeschneidung festhalten. Es kann lediglich vermutet werden, dass diejenigen, „die immer noch an die Sache glauben“, Wege finden, ihre Töchter beschneiden zu lassen. Ob diese Frauen den Eingriff dann in der Schweiz oder in Somalia durchführen lassen, weiß Nadifa nicht. Sie weiß auch nicht, ob diese Frauen sich aufgrund des bestehenden Verbotes von Mädchenbeschneidung in der Schweiz, gegen den Eingriff entscheiden.

In diesem Interviewabschnitt hebt Nadifa jedoch einen ihrer Meinung nach zentralen Aspekt hervor. Sie spricht von ihren Freundinnen als „die besser ausgebildeten Frauen“, die Mädchenbeschneidung ablehnen. Für Nadifa scheint Bildung und die Entscheidung gegen Mädchenbeschneidung eng miteinander gekoppelt zu sein. Diesen Eindruck vermittelt sie, indem sie dies so erzählt, als handle es sich um eine Selbstverständlichkeit, dass sich besser ausgebildete Frauen, aus Nadifas Sicht „Frauen, die hier ihr Leben so meistern“, gegen Mädchenbeschneidung entscheiden würden. „Frauen, die ihr Leben hier so meistern" könnte als Unabhängigkeit gelesen werden. Möglich ist, dass Nadifa damit zum Ausdruck bringen möchte, dass dies Frauen sind, die ihr Leben unabhängig von sozialem Druck (sei es einen Ehemann oder einer Familie, die Mädchenbeschneidung fordert) meistern können. Damit verbunden ist vermutlich auch die finanzielle Unabhängigkeit, da diese den Frauen ermöglicht, sich gegebenenfalls von einer Familie trennen zu können, die Mädchenbeschneidung einfordert. Schlussfolgernd bedeutet dies, dass Nadifa der Meinung ist, dass (Aus-)Bildung Frauen zur Unabhängigkeit verhilft und dazu beiträgt, dass sich diese Frauen gegen Mädchenbeschneidung entscheiden. Nadifa verkehrt mit solchen Frauen, die genau wie sie selbst ihre Töchter nicht beschnitten haben. Zu Frauen, die anderer Ansicht sind, hat sie keinen Kontakt.

\subsubsection{Medizinisches Fachpersonal in der Schweiz}

Da Nadifa keinen Bedarf von Aufklärungsarbeit unter somalischen Migrant innen zu Beschneidung in der Schweiz sieht, frage ich sie nach der Notwendigkeit besonderer Schulungen von Ärzt innen und Hebammen im Umgang mit Beschnittenen. Diesbezüglich ist sie der Meinung, dass es mittlerweile mehr Ärzt_innen gebe, die über Beschneidung Bescheid wüssten als noch vor 18 Jahren, als sie in die Schweiz immigrierte. Trotzdem spricht sie sich deutlich für noch mehr aufgeklärtes medizinisches Fachpersonal aus.

$\mathrm{N}$ : „Weil es kommen Frauen mit Beschneidungen, die ihre Kinder zur Welt bringen, in den Spitälern. Es kommen junge Frauen, die ähm, mit der Periode oder IRGENDETWAS Probleme haben. Und die Ärzte oder die Hebammen oder halt die Leute, die in diesem Bereich arbeiten, müssen BEsser aufgeklärt werden, damit sie damit BEsser umgehen und der Patient das 
Gefühl hat „Ok, ich bin ein Mensch, ich bin normal, ich bin wie eine normale Frau hier.“ Und nicht das „Oh, was ist das?“ und „Oje, wie ist das geschehen?" und „Warum?“ $\{$ ggenervt\}\}, einfach all diese Fragerei sollte es nicht MEHR geben“ (I 2, 201-207)

Nadifa zögert kurz bevor sie antwortet, spricht sich dann aber dafür aus, medizinisches Fachpersonal im Umgang mit beschnittenen Mädchen und Frauen zu schulen. Um gewährleisten zu können, dass künftig Beschnittene kundig versorgt würden, sollten aus ihrer Sicht Mediziner_innen und Hebammen soweit aufgeklärt werden, dass keine Fragen mehr nötig sind und sie beschnittenen Frauen den Eindruck vermitteln können, wie Unbeschnittene zu sein. Nadifas Wunsch ist, dass beschnittene Frauen auf medizinisches Fachpersonal treffen, das nicht länger irritiert oder schockiert auf beschnittene Genitalien reagiert. Aufklärung dieser Personengruppe bedeutet aus Nadifas Sicht, sie mit beschnittenen Genitalien und den Hintergründen der Praktik derart vertraut zu machen, dass sie fähig sind, Beschnittene genauso unaufgeregt zu behandeln wie Unbeschnittene.

Während Nadifa an anderen Stellen direkt und unverblümt antwortet, ist sie an dieser Stelle kurz zögerlich, muss erst einmal nachdenken. An anderen Stellen im Interview kommt es zu Gesprächspausen, wenn sie nach dem passenden Wort sucht. Hier erscheint es als müsse sie abwägen, ob sie dieser Frage zustimmen darf. Nadifa spricht sich während des gesamten Interviews gegen die Einmischung, europäischer ${ }^{6}$ oder ,schweizerischer ${ }^{6}$ Organisationen/Personen in das Thema weiblicher Genitalbeschneidung aus. An dieser Stelle könnte daher eine Lesart des Zögerns sein, dass sie anfangs kurz dazu tendierte die Frage nach der Wichtigkeit aufgeklärter Ärzt_innen/Hebammen zu verneinen. Dann aber nutzt sie die Frage, um noch einmal deutlich zu machen, dass auch schweizerische Ärzt innen und Hebammen lernen müssen, wie ein sensibler und respektvoller Umgang mit beschnittenen Frauen aussehen soll. Sie räumt zwar ein, dass der medizinische Umgang mit Beschnittenen heute sensibler vonstatten geht als noch vor 18 Jahren, doch werden Beschnittene noch immer nicht wie Unbeschnittene behandelt.

\subsubsection{Zusammenfassung}

Nadifa macht von Anfang an zwei Gruppen auf: die Gruppe „der Somalier“ und die ,der Europäer“. Sie selbst ordnet sich in dieser Gruppenaufteilung eindeutig „den Somaliern“ zu, die sie aber nochmals unterteilt in „die Somalier", die in Somalia geblieben sind und in diejenigen, die wie sie in der Schweiz wohnen. Aus diesem Blickwinkel heraus führt sie das Interview, indem sie besonders deutlich macht wie sehr sie „die Europäer" für ihren Umgang mit „,den Somaliern“ ablehnt. Für Nadifa handelt es sich um einen von Überheblichkeit und Bevormundung geprägten Umgang, den sie noch 
gut aus ihrem Leben in Somalia kennt. Letztlich beschreibt Nadifa die Folgen des Kolonialismus, die ihrer Meinung nach noch bis heute den Umgang ,der Europäer“ mit „den Somaliern“ prägen. Nadifa ist darüber sehr verärgert. Ihre Strategie, sehr verallgemeinernd über ganze Bevölkerungsgruppen und deren Haltung zu sprechen, schützt sie vor Personen, die ihr in der von ihr beschrieben Art und Weise begegnen. Die Kehrseite ist, dass sie den Blick für Personen verliert, die ihr wertschätzend und offen gegenübertreten. Auch die Arbeit gegen Mädchenbeschneidung betrachtet sie aus dieser Perspektive heraus und zieht den Schluss, dass „die Europäer“ in erster Linie lernen müssten, wie sie mit „den Somaliern“ umzugehen hätten. Nadifa zweifelt jedoch daran, dass dies gelingen kann. Sie spricht sich dafür aus, dass somalische Frauen die Arbeit gegen Mädchenbeschneidung übernehmen müssten, allerdings müsse diesen auch erklärt werden, dass Verbote oder Drohungen kontraproduktiv seien. Stattdessen plädiert sie für eine Aufklärung, in der den Menschen auf Augenhöhe begegnet und erklärt wird, warum Mädchenbeschneidung keine Vorteile hat. In Bezug auf das medizinische Fachpersonal betont sie dasselbe: In erster Linie müssten sie im sensiblen Umgang mit Betroffenen geschult werden und lernen, dass Beschnittene so ,normal' seien wie Unbeschnittene.

\subsection{Ayaan}

Ayaan ist in einer kleinen Stadt im Norden Somalias geboren, wuchs jedoch in einer anderen somalischen Stadt auf und lebte dort bis sie 20 Jahre alt war. Mit 19 Jahren hat sie in Somalia ihr erstes Kind entbunden. Zu diesem Zeitpunkt war sie noch mit ihrem ersten Ehemann verheiratet. Mit 20 Jahren ist sie nach Saudi-Arabien gezogen, wo sie als Krankenschwester in einer Universitätsklinik arbeitete, ihren zweiten Ehemann kennen lernte, sich scheiden ließ und diesen heiratete. Heute haben sie fünf gemeinsame Kinder und leben in England. Ihre Tochter aus erster Ehe hat sie in Somalia bei ihrem ersten Ehemann und dessen Familie zurück lassen müssen - so sei das Gesetz in Somalia: Der Vater habe das alleinige Sorgerecht und bestimme nach einer Trennung über die gemeinsamen Kinder, erklärt Ayaan.

Von der Zeit in Saudi-Arabien berichtet Ayaan sehr positiv, betont dort viel Geld verdient und deshalb ein eigenständiges und finanziell gesichertes Leben geführt zu haben. In Saudi-Arabien entband Ayaan ihr zweites Kind. Insgesamt lebten sie, ihr Ehemann und ihr Kind acht lang Jahre dort bis sie mit circa 28 Jahren wieder zurück nach Somalia zog. Ihr Ehemann blieb in Saudi-Arabien, da er dort Arbeit hatte, so lange bis er in England Arbeit gefunden hat, wo sie heute zusammen wohnen. Zurück in Somalia wurde Ayaan schnell bewusst, dass die aufgrund des Krieges nicht bleiben konnte, 
besonders weil sie zu diesem Zeitpunkt bereits ihr drittes Kind erwartete. Sie entschied sich dafür, nach England zu gehen, in der Hoffnung, baldmöglichst wieder zurück in ihre Heimat gehen zu können. Da dies jedoch nicht der Fall war, beantragte sie Asyl und blieb. Diese Zeit beschreibt Ayaan als sehr schwierig, da sie plötzlich all ihren Besitz und ihr finanziell gesichertes Leben aufgeben musste. Mittlerweile ist sie 51 Jahre alt, lebt seit vielen Jahren in einer Großstadt Englands und arbeitet als Krankenschwester. Da bei diesem Interview nach circa 15 Minuten die Batterien des Aufnahmegeräts leer waren, ist der letzte Teil des Interviews ein Erinnerungsprotokoll, das direkt im Anschluss an das Interview angefertigt wurde, woraus jedoch nur die Elemente verwendet werden, die in Bezug auf die Thematik noch nennenswert sind.

\subsubsection{Weibliche Genitalbeschneidung in Somalia}

Ayaan beginnt das Interview mit der Erzählung von ihrer eigenen Beschneidung damals in Somalia. Sie berichtet ähnlich wie Sara, dass sie am Tag ihrer Beschneidung nicht wusste, was auf sie zukommen würde:

A: "No, I don't know what happen? I know the consequences, that it's something (--) anyone mother who loves her daughter is doing it, so it is something important to do it. So, but I don't know that it will have impact on me, on my feelings, for my future, when I'm having babies, or all like that." (I 3, 15-18)

Sie erzählt, dass Eltern, die ihre Töchter lieben, dazu verpflichtet sind, sie beschneiden zu lassen, auch weil es wichtig sei, diesen Eingriff durchzuführen. Sie als Mädchen habe damals jedoch nicht gewusst was es bedeuten würde beschnitten zu werden, niemand habe sie darüber informiert, welche Auswirkungen der Eingriff auf ihre Gefühle, ihre Zukunft oder die Geburt ihrer Kinder haben würde. Trotz der Auswirkungen, von denen sie im Anschluss sehr detailliert berichtet, erzählt Ayaan sachlich über den Tag der Beschneidung und dessen Konsequenzen auf ihr Leben. Sie spricht davon, dass Mädchenbeschneidung in Somalia eine unhinterfragte Norm darstelle, gemäß der sich Eltern aus Liebe zu ihren Töchtern verhielten.

Auch die Mädchen gingen davon aus, dass es sich bei diesem Ereignis um einen wichtigen Schritt in ihrem Leben handle, der für sie positiv konnotiert sei:

A: "When I grew up circumcision was something privileged, it was something back home and if you are not circumcised, children will bring you up in school, so then you better have to do it, cause it's something privileged, something very good there." (I 3, 10-13)

Mithilfe des Eingriffs würden Eltern zeigen, dass sie privilegiert seien, zwei Mal betont Ayaan dies. Daraus kann gelesen werden, dass die Mädchen 
froh sein können, wenn sie diesem Eingriff unterzogen werden. Ayaans Erwähnung der Schule könnte dahingehend interpretiert werden, dass die Mädchen in der Schule unter Druck gesetzt werden, wenn sie noch nicht beschnitten sind, was sich mit der Erzählung Saras decken würde. Bestärkt wird diese Vermutung durch den Zusatz Ayaans ,you better have to do it", was klingt als sei es eine Verpflichtung, die keine Wahlmöglichkeit offen lässt. Die negativen Konsequenzen des Eingriffs, während der Beschneidung und auch danach, würden dagegen nicht thematisiert. Nur über die positive Seite wird gesprochen und den Mädchen suggeriert, dass es sich um ein Privileg handelt und sie stolz und froh sein sollen. Ayaan erzählt dies, ohne dass der Eindruck entsteht, sie würde den Eingriff rechtfertigen wollen, es klingt mehr wie eine nüchtern betrachtete Erklärung. Ayaan selbst lehnt Mädchenbeschneidung ab, doch zeigt sie sich in weiten Teilen des Interviews, besonders in den Passagen zu Somalia und der Bedeutung der Beschneidung dort, emotionslos. Diese Emotionslosigkeit macht den Anschein, als habe sie wenig Hoffnung, dass Mädchenbeschneidung in Somalia beendet werden könne, da sie nun einmal gemacht würde.

Als Ayaan fortfährt vom Tag ihrer Beschneidung zu berichten, erzählt sie nicht, wie viele andere Mädchen, in einer Hütte von einer Beschneiderin beschnitten worden zu sein. Ihre Eltern hätten sie in ein Krankenhaus gebracht und unter Narkose beschneiden lassen, dort sei ihr ,nur ${ }^{6}$ die Klitoris entfernt worden, nicht aber die kleinen und großen Labien. Sie wurde jedoch, so wie es in Somalia üblich ist, bis auf eine kleine Öffnung vernäht - ein Schritt, der in Somalia laut Ayaan von großer Bedeutung ist, warum erklärt sie im Anschluss:

A: "So that's why they do it, because it's beauty for the girls, when they get married for the husband to see, that [she was not playing around]"

I: "[that he is the first one]"

A: "Yeah, the first one, ja. Those who do it, playing around, they have to sew themselves, so it's a verification that you are virgin, they don't know that the virgin is somewhere else, at that time it's so. And if you are married and husband find out that you are not sewed then he, they will think that you played around or something. And then you get divorced." (I 3, 29-36)

Der Naht kommt in der Hochzeitsnacht besonderes Gewicht zu, da eine intakte Naht ein wichtiger Beweis für die Jungfräulichkeit der Braut darstellt. Für den künftigen Ehemann wäre die verschlossene Naht eine Art Beweisstück dafür, dass seine zukünftige Ehefrau nicht vorehelichen Geschlechtsverkehr gehabt hatte. Würde in der Hochzeitsnacht festgestellt, dass die Frau vorehelichen Geschlechtsverkehr gehabt hat, würde es nicht zu einer Eheschließung kommen, berichtet Ayaan. Für Frauen, die vorehelichen Geschlechtsverkehr gehabt hätten, bedeutet dies, sich selbst wieder zunähen zu müssen. Die Naht ist entscheidend: Ist diese verschlossen, gilt die Frau als Jungfrau - unabhängig davon, ob sie Geschlechtsverkehr hatte oder nicht. 
Geschlechtsverkehr scheint auch auf vaginalen Verkehr reduziert zu sein, weitere sexuelle Handlungen bleiben somit ausgeklammert. Sobald die verschlossene Naht für beschädigt empfunden wird, führt das dazu, dass die betroffene Frau nicht geheiratet wird. Der Bericht Ayaans lässt vermuten, dass die Entscheidung, welche Naht als verschlossen gilt und welche nicht, nicht grundsätzlich zu bestimmen ist, so dass die Frauen großer Willkür ausgesetzt sein können. Des Weiteren bleibt offen, wie mit Frauen umgegangen wird, die gewaltsam zum Geschlechtsverkehr gezwungen wurden und aus diesem Grund keine verschlossene Naht mehr ,vorweisen' können. Ayaans Erzählungen verdeutlicht, dass die Naht der Beschneidung an die Stelle des Jungfernhäutchens gerückt ist, geht aber nicht darauf ein, wie dieser Wandel zustande kam. Vermutlich ist eine verschlossene Naht leichter kontrollierbar als ein feines Häutchen, das zu erkennen anatomischer Vorkenntnisse bedarf.

Nachdem in der Hochzeitsnacht festgestellt wurde, dass die Naht verschlossen ist, komme laut Ayaan dem Ehemann die Aufgabe zu diese zu öffnen.

A: "Then, after that what happens is, that the male has to perform, in southern parts the man has to do it, cause that shows that the man is strong and healthy, otherwise if he gets support from a medical, äh, health professionals, I mean, than it is not, than he gets the feeling, that he is weak for his erection or something like that, so he, he feels this is failure, so he wants to show that, without support he can penetrate the girl, that's what in southern parts passes, not in the northern parts." (I 3, 65-70)

Für eine infibulierte Frau bedeutet somit der erste Geschlechtsverkehr, in eine kleine Öffnung penetriert zu werden, die in der Regel zu klein ist, um eine natürliche Penetration zu ermöglichen. Ayaan erzählt von der Herausforderung, die dies für den Mann bedeutet, wie sehr dieser unter Druck stehe, da ihm die Aufgabe zukomme, ohne Hilfsmittel, allein mit seinem erigierten Glied den vaginalen Geschlechtsverkehr zu vollziehen. Gelingt ihm das, so sei das ein Beweis für seine Stärke und seine Gesundheit, benötigt er aber die Hilfe eines Arztes, gelte er als schwach oder zu wenig sexuell erregt. Im Süden Somalias sei dies laut Ayaan ein Anreiz für jeden Mann es ohne Hilfsmittel zu probieren. Ayaan erzählt auch diese Geschichte emotionslos. Sie berichtet auch nur von den Folgen, die diese Regelung für den Ehemann haben, und geht mit keinem Wort darauf ein, welche Folgen sich daraus für die Ehefrau ergeben. Dieses Nichterwähnen könnte dahingehend gelesen werden, dass es für die Frauen ein zu ertragendes Los ist, etwas, dass es nicht lohnt zu hinterfragen oder sich darüber zu beklagen. Frauen haben die Aufgabe, die Konsequenzen der Infibulation zu ertragen und mit dem gewaltsamen Öffnen der Naht umzugehen. Dieser Eindruck bestärkt sich, als Ayaan ähnlich gelassen von ihrer eigenen Hochzeitsnacht berichtet. Ayaan ist weder verärgert darüber, große Schmerzen ausgehalten zu haben, noch ist sie traurig, einem derart folgenreichen Eingriff ausgesetzt worden zu sein. Mittler- 
weile sind sehr viele Jahre vergangen, so dass eine Lesart ihres gelassenen Berichts sein könnte, dass sie heute genug Abstand zu Geschehenem gewonnen hat und deshalb sachlich über das ihr widerfahrene Leid berichten kann. Möglich ist aber auch, dass sie ihre Geschichte nur erzählen kann, wenn sie keinen emotionalen Zugang zu ihrem Erleben zulässt, weil sie sonst Gefahr laufen würde von ihren Gefühlen überwältigt zu werden.

\subsubsection{Zu den Folgen der Infibulation}

Nachfolgend berichtet Ayaan von den Auswirkungen, die ihre eigene Beschneidung für sie hatte. In diesem Zusammenhang legt sie den Fokus auf die Konsequenzen, die Genitalbeschneidung bei der Entbindung ihrer Kinder hatte.

A: "So then when I'm married I faced all these difficulties of the southern parts, I come from southern parts, Nur comes from northern parts"

I: "Ok."

A: "of Somalia. So the delivery it was hard, it was very hard"

I: "I can imagine"

A: "Although the midwives in Somalia they are well trained how to do it before anything happens. When the women are pushing, they will hold the cicatrise and then they will start [cutting]"

I: "[cutting]"

A: "By later, after that they have to sew it as it was again, they will sew it as it was again (---)" (I 3, 78-88)

Ohne zu beschönigen erzählt Ayaan was sie als beschnittene Frau während und nach der Geburt ertragen musste: das Aufschneiden und wieder Zunähen der Naht nach jeder Entbindung. Die Hebammen in Somalia seien zwar auf die erschwerte Geburtssituation vorbereitet und könnten professionell damit umgehen, doch führe laut Ayaan kein Weg am Öffnen der für die Entbindung zu kleinen Öffnung vorbei. Die nach der Beschneidung verbliebene Öffnung muss durch Schnitte vergrößert werden, erst dann könne der Kindskopf austreten. Danach folge das erneute Zunähen und der ewige Kreislauf wiederhole sich mit jeder Schwangerschaft beziehungsweise Geburt. Ayaan betont, dass Geburten für beschnittene Frauen hart, sogar sehr hart seien und räumt mit dieser Aussage für einen Moment ein, dass Frauen aufgrund der Infibulation mit schweren Folgen leben müssen. Indem sie aber direkt im Anschluss die Kompetenzen der Hebammen im Umgang mit einer infibulierten Frau unter Geburt in den Vordergrund stellt, bleibt der Eindruck, dass Frauen aus Ayaans Sicht dafür bestimmt sind, mit den Schwierigkeiten zu leben. Wichtig sei nur, dass bei der Entbindung informierte Hebammen zur Verfügung stehen. Am Ende der Geburt würden die Frauen wieder zugenäht, so sei es nach jeder Entbindung. 
Ayaan geht im Anschluss auf ihre Entbindungen ein. Wie sie am Anfang des Abschnittes sagt, hat sich für sie das Ausmaß der negativen Konsequenzen ihrer Beschneidung erst während und nach den Geburten ihrer Kinder gezeigt.

A: "So I was, I had difficult labours, my baby was $4 \mathrm{~kg}$, I was 19 years old when I had my first baby (lächelt) and it was very hard, so they give my three injections left and right and after they cut for me so then they stitched it again. Then I had to take antibiotics back home, so maybe the surgery thing they do is not very sterile, so you can get infections. And those infections, if you have had your first baby then after that you can not have any other babies. Because these infections [you maybe go to...]"

I: "[infertility]"

A: "infertility, yes. So most of the women who had their first baby the second baby is difficult. The second baby or nothing! (---) Cause the infection rate is high, the delivery, sutures, they will give you anti-, they gave me antibiotics, ten days antibiotics intramuscular. This was so painful I couldn't sleep on any side, I couldn't sit, because my bottom was hurting. And my husband was, he was ( ) faculty of ( ) science, so I had serve waitresses maybe to look after me, and somebody who was holding the baby, because I cannot get up." (I 3, 98-110)

Ayaan war 19 Jahre alt als sie ihr erstes Baby bekam, aus ihrer Sicht ein schweres Kind, das ihr eine schwere Geburt bescherte. Sie musste von mehreren Seiten geschnitten und im Anschluss wieder zusammen genäht werden. Nach der Geburt habe sie eine Infektion bekommen, die mit Antibiotika behandeln werden musste. Offen bleibt, ob die Operation in einer Klinik unter sterilen Bedingungen gemacht wurde oder ob sie zuhause entbunden hat und die unsterilen Bedingungen, unter denen die Operation erfolgte, für die folgende Infektion verantwortlich zu machen sind. Sie spricht von Operationen, die „strong“ sind, also „,harten“ Operationen, die zu Infektionen führen können. Wie sie „hart" in diesem Kontext interpretiert, bleibt ungeklärt. Für Ayaan steht aber fest, dass ihre Infektion eine Folge der Operation war. Im letzten Satz spricht sie ganz allgemein und weist darauf hin, dass die Infektionsrate hoch sei. Frauen hätten nach der ersten Entbindung und den damit verbundenen Infektionen Angst, keine weiteren Kinder mehr bekommen zu können. Dieser allgemein gehaltene Hinweis zum Thema Infektionen liest sich so, als wolle sie unterstreichen, dass ihr nichts Ungewöhnliches widerfahren sei und Infektionen schlicht dazu gehörten, nichts worüber man besonders verwundert oder aufgebracht sein müsse. Die Angst vor Sterilität aufgrund der hohen Infektionsrate, ist das einzige Gefühl, das sie in diesem Zusammenhang benennt. Die Frage, ob nach solch einer schweren Geburt mit Infektionsfolge noch weitere Kinder gewollt sind, hat sich Ayaan entweder nicht gestellt oder sie steht nicht zur Wahl. Neben der Infektion, kämpfte 
Ayaan nach der ersten Entbindung mit starken Schmerzen und einer zu eng zusammen genähten Naht, die sie sehr einschränkte.

A: "The pain and the tidiness of fissure, ja?"

I: "Mmh." [...]

A: "It's other, it's like oppression. So they have to wash and it takes a long time to heal. So it was so painful, you cannot sit, you cannot give the baby breastfeeding the next day. So that's the experiences in Somalia I had. Then I moved to South Arabia I was working as a nurse there. I was working at the, you can call the UniversityHospital." (I 3, 107 -118)

Nach der Entbindung habe sie weder schlafen noch sitzen können, so stark seien die Schmerzen gewesen. Hinzu kam, dass sie mit dem Kind alleine war und sie eine Hilfskraft beschäftigen musste, weil sie nicht im Stande war, aufzustehen, um ihr Kind zu versorgen. Sie erzählt, zu eng zusammen genäht worden zu sein, was sie einschränkte. Außerdem habe die Wunde lange Zeit gebraucht um zu heilen. Ayaan erzählt ihre Geschichte detailliert, als wolle sie sicher gehen, dass deutlich wird was es bedeutet, als beschnittene Frau zu entbinden. Trotz der Details, ähnelt ihre Erzählung nach wie vor einem Bericht, da sie auch die genannten Emotionen nicht in den Vordergrund stellt. Es klingt so, als würde sie gar nicht von sich selbst erzählen.

Auch im weiteren Verlauf des Interviews spielt ihr eigenes Erleben eine untergeordnete Rolle. Im Vordergrund stehen die physischen Schwierigkeiten, die der Eingriff für sie hatte: schwierige Geburten, die damit verbundenen Schmerzen und die langwierige Heilung als medizinische Konsequenzen ihrer Infibulation. $\mathrm{Zu}$ psychologischen Auswirkungen äußert sie sich nicht. Stattdessen fährt sie fort mit den weiteren Entbindungen, die sie je nach dem in welchem Land sie zu dem Zeitpunkt lebte, als schwieriger und weniger schwierig beschreibt. Während sie die Geburt ihrer Kinder in Somalia und Saudi-Arabien zwar als "schwere" Geburten beschreibt, empfindet sie die Geburten in England aufgrund des unwissenden Fachpersonals am schwersten.

A: "Then I had the second baby. When I had the second baby, it was my $4^{\text {th }}$ one, you know. So I wrote that for the nurses, I told that the midwives, so please I need a C-section. So, a lady who can have FOUR babies and ASK for C-section? It's surprising a midwife maybe, so she didn't know. So I asked her to make me an C-section, I wrote it in my writing, but she didn't listen to me. So I was wondering, those people who cannot write it, so I told her what I needed to be done. She DIDN'T listen to ME." (I 3, 181-186)

In England seien die Geburten ,very difficult“ gewesen, da das Klinikpersonal nicht gewusst habe wie beschnittene Frauen zu öffnen seien, um eine vaginale Entbindung zu ermöglichen. Dies habe sie nach der ersten Geburt in England schon gewusst. Aus diesem Grund habe sie für die zweite Entbindung (ihrem insgesamt vierten Kind) nach einem Kaiserschnitt verlangt. Die Hebammen hätten aber nicht auf sie gehört, weil sie der Meinung waren, dass 
eine Frau, die bereits drei Kinder vaginal entbunden hatte, auch das vierte Kind vaginal entbinden könne. Außerdem erzählt Ayaan weiter, habe man sie im Anschluss an die Geburt nicht wieder zugenäht, was zu weiteren Komplikationen geführt habe. An dieser Stelle zeigt sich Ayaan verwundert über die Hebammen, da diese nicht auf sie gehört hätten. Nicht der Umstand, durch die Infibulation so viele schwere Geburten erleben zu müssen, verwundert sie, sondern die Tatsache, dass die Hebammen nicht auf sie hörten.

Im weiteren Verlauf des Interviews, der ab dieser Stelle aus dem Gedächtnisprotokoll entnommen ist, ist sie irritiert darüber, nicht als Expertin ihrer Situation wahr- und ernstgenommen worden zu sein. Schließlich habe sie bereits Erfahrungen im Kinder gebären gehabt und sei außerdem Krankenschwester, die auch aufgrund ihrer medizinischen Kenntnisse wisse, was zu tun sei. Dennoch hätten die Krankenschwestern und Hebammen bei der Entbindung des vierten Kindes nicht auf sie gehört, das wiederholt sie mehrmals. Ayaan habe versucht zu erklären, aufzuschreiben was mit ihr unter Geburt gemacht werden solle, trotzdem habe niemand auf sie gehört. Zum ersten Mal im Interview scheint dies Ayaan außer Fassung zu bringen. Sie erhebt erstmals die Stimme, berichtet laut davon, einfach nicht gehört und nicht ernst genommen worden zu sein. Ayaan erzählt weiter von den negativen Erfahrungen, die sie mit den britischen Hebammen und Ärzt_innen gemacht habe, was sie darauf zurück führt, dass sie keine Erfahrung im Umgang mit infibulierten Frauen hätten. Hinzu käme aber, und das ärgert Ayaan, dass sie nicht bereit gewesen seien, auf sie als Betroffene und erfahrene Frau zu hören. Nach der vierten Entbindung habe sie gesagt, dass sie wieder zusammengenäht werden müsse, was ihr verweigert wurde. Ayaan erzählt, dass sie daraufhin sehr viel geblutet habe, dass es gar nicht mehr aufgehört habe zu bluten. Sie sei mehrfach in der Klinik gewesen mit dem Verweis, dass sie sich auskenne und wisse, dass es erst aufhören würde zu bluten, wenn sie sie zusammen nähen. Doch erst als sie auf eine somalische Ärztin traf wurde ihr zugehört und geholfen.

Nach dem vierten Kind wurde ihr von den Ärzt_innen geraten, keine weiteren Kinder mehr zu bekommen. Trotz der vielen schweren Entbindungen und den damit verbundenen Konsequenzen wollte Ayaan noch ein letztes Kind. Sie begründet diese Entscheidung damit, bis zu diesem Zeitpunkt ,nur ${ }^{6}$ einen Sohn gehabt zu haben, sich aber einen weiteren Sohn gewünscht habe. Sie bekam den gewünschten Sohn und ließ sich nach der Geburt des fünften Kindes sterilisieren. Unabhängig von den Schmerzen rund um ihre Entbindungen sei sie heute froh, fünf Kinder zu haben. 


\subsubsection{Zusammenfassung}

Ayaans Interview verdeutlicht sehr detailliert, mit welchen Konsequenzen infibulierte Frauen leben müssen. Sie selbst scheint einen Umgang mit den wiederkehrenden Schmerzen und den Infektionen gefunden $\mathrm{zu}$ haben, der darin zu bestehen scheint, sich wenig von ihren Gefühlen leiten zu lassen, sondern den Fokus auf die physischen Konsequenzen und dem medizinischem Umgang damit zu legen. An ihrem eigenen Beispiel beleuchtet sie, wie bedeutsam Hebammen sind, die im Umgang mit infibulierten Frauen Erfahrung haben. Sie wüssten wo geschnitten werden müsse, damit eine vaginale Entbindung möglich wird. Anschließend würden sie die Wunde wieder zusammen nähen und damit gewährleisten, dass die Frauen nicht zu viel bluteten, was aus Ayaans Sicht sinnvoll ist. Ayaan betrachtet weibliche Genitalbeschneidung aus Sicht der Krankenschwester, die sie ist. Die medizinischen Folgen einer Infibulation sind ihr vertraut, ebenso wie die medizinische Begleitung beschnittener Frauen. Ihre Ausbildung hilft ihr, mit den physischen Konsequenzen des Eingriffs zu leben und diese zu akzeptieren. Es bleibt der Eindruck als habe Ayaan sich schon lange mit ihrem Schicksal abgefunden, als beschnittene Frau durchs Leben zu gehen und all die daraus resultierenden Folgen hinzunehmen. Emotionen zeigt sie erst, als sie davon berichtet, von Hebammen in England nicht gehört worden zu sein. Es ist der ihrer Meinung nach unprofessionelle Umgang dieser Hebammen mit einer infibulierten Frau, der bei Ayaan Ärger auslöst - nicht aber die Tatsache, als Mädchen infibuliert worden zu sein und noch bis heute mit den schweren Folgen des Eingriffs leben zu müssen. Ihre beschnittenen Genitalien gehören $\mathrm{zu}$ ihr, sie beklagt sich nicht über die dadurch entstandenen Schmerzen, sie lässt es sich trotz erschwerter Umstände auch nicht nehmen, Kinder zu bekommen. Aus Ayaans Sicht scheint die Hauptsache zu sein, als infibulierte Frau medizinisch professionell versorgt zu werden und dazu gehört medizinisches Fachpersonal, das weiß, wie sich der Umgang mit infibulierten Frauen gestaltet.

\subsection{Layla}

Layla wurde von der nach Italien mitgereisten Dolmetscherin in einem somalischen Lebensmittelgeschäft in Rom angesprochen und gefragt, ob sie bereit wäre mit uns in einem Café über Beschneidung zu sprechen. Layla willigt sofort ein und geht mit in ein nahe gelegenes Café. Sie ist Ende 20 Jahre alt, ist aus ihrer Heimat über den Sudan nach Libyen geflohen, wo sie ein Jahr lebt und weiter nach Lampedusa in Italien flieht. Seit 2004 lebt sie in Italien. 
Layla erzählt viel und sehr schnell, springt in ihren Erzählungen von einem Thema zum nächsten und erzählt immer wieder nur kurze Sequenzen. Sie spricht so schnell, dass meine Dolmetscherin an vielen Stellen nachfragen muss, weil der Zusammenhang missverständlich ist. Trotzdem bleibt am Ende des Interviews, selbst nach wiederholtem Lesen der deutschen Übersetzung einiges unklar.

Layla äußert zu Beginn des Gesprächs ausschließlich Italienisch sprechen zu wollen, obwohl meine Dolmetscherin ihr anbietet auch auf Somali kommunizieren zu können. Layla gibt an, nicht Somali sprechen zu wollen, aus Angst als Zugehörige eines Unterclans identifiziert $\mathrm{zu}$ werden. Aufgrund ihres somalischen Dialektes könne heraus gehört werden, dass sie einem Subclan angehöre, was für sie zur Folge hätte feindlichen Angriffen innerhalb der somalischen Flüchtlinge und Migrant_innen in Rom ausgesetzt zu sein.

\subsubsection{Ein Leben in Angst, Abhängigkeit und Schmerz}

Layla gibt an, in Lampedusa bei einer Frau gewohnt und gearbeitet zu haben, die sie jedoch nie mit Namen benennt. Sie spricht nur von „der Frau“ und erzählt, dass sie bei ihr im Haus wohnen durfte, dies aber an die Bedingung geknüpft gewesen sei, für sie arbeiten zu müssen. Vieles aus der Zeit in Lampedusa bleibt unklar: was Layla gearbeitet hat, ob sie dort die einzige (somalische) Arbeitskraft war, ob „die Frau“ ebenfalls somalischer Herkunft war und wie sie „,der Frau“ begegnet ist. Wir erfahren nicht, ob Layla Geld für ihre Arbeit bekommen hat oder ob sie dafür im Haus „der Frau“ wohnen durfte und Nahrung bekommen hat.

Die Beschreibung der Abhängigkeit von „,der Frau“ und der Angst vor dem Verlust der Arbeitsstelle dominieren Laylas Anfangserzählung. Besonders deutlich wird Laylas Abhängigkeit als sie davon erzählt, „die Frau“ um Hilfe gebeten zu haben, da sie unter ständig Unterleibsschmerzen litt. „Die Frau“ habe ihr gesagt, dass es niemanden gäbe, der ihr helfen kann, und Layla lieber ihrer Arbeit nachkommen solle, bevor sie sich eine gesunde Arbeitskraft nehme. Layla erzählt, dass ihr diese Drohung Angst gemacht habe, dass ihr „die Frau“ auch gesagt habe, sie müsse Italien verlassen, sofern sie keine Arbeitsstelle habe. Da es aus Laylas Sicht keinen Ausweg aus dieser Situation gibt, entscheidet sie, die Schmerzen zu ertragen und bei „der Frau“ zu bleiben. Das Leben bei „der Frau“ und unter andauernder Schmerzen scheint Layla erträglicher als wieder zurück nach Somalia zu müssen. Als „die Frau“ 2010 stirbt verlässt Layla Lampedusa und geht nach Rom. Nachdem Layla in Rom angekommen ist, berichtet sie Folgendes:

L: „Nachdem sie gestorben ist, bin ich krank geworden und bin im Krankenhaus Columbus operiert worden. Sie haben mir etwas ausgeschnitten unten, ich hatte Gebärmutterhalskrebs. Ich kann gar nicht mehr arbeiten, ich 
habe ständig da unten Schmerzen. Ich habe keine Adresse, ich wohne in einem kaputten Haus, ich habe nicht einmal was zu Essen, ich bin überall gewesen, niemand hilft mir. Sie wollen nicht mal meinen Aufenthalt erneuern. Du musst eine Arbeit finden oder zurück in deine Heimat, sagen sie mir.“ (I 4, 8-13)

Layla erzählt von ihrer Gebärmutterhalskrebserkrankung und der nötigen Operation. Aus ihrer Sicht ist sie erst nach dem Tod „der Frau“ erkrankt, die Tatsache, bereits zu Lebzeiten ,der Frau“ nach medizinischer Hilfe im Umgang mit ihren Schmerzen gefragt zu haben, bewertet Layla scheinbar nicht als ,krank'. Vermutlich trifft für Layla der Begriff ,krank ${ }^{6}$ erst zu, wenn sie medizinische Leistungen (Untersuchung, Diagnose und Operation) in Anspruch nehmen muss. Sie lässt sich in Rom operieren, doch begleiten sie ihre Unterleibsschmerzen nach wie vor, so dass sie bis heute keiner Arbeit nachgehen kann. Arbeitslos zu sein belastet Layla sehr, da „sie“ ihr gesagt hätten, dass sie ohne Arbeit zurück nach Somalia müsse - so wie bereits „die Frau“. Wer „sie“ sind, wird nicht deutlich: die Mitarbeiter_innen der Auslandsbehörde, andere Somalis oder Asylbewerber_innen. In jedem Fall ist Layla davon überzeugt, ohne Arbeit Italien verlassen zu müssen. Layla spricht sehr schnell und etwas abgehakt, sie wirkt während des ganzen Interviews wie eine Getriebene, eine, die nie zur Ruhe kommt. Gleichzeitig macht sie einen verzweifelten Eindruck, zieht am Ende des Abschnittes das Resümee: „niemand hilft mir.“

Sie gibt aber nicht auf, begibt sich immer wieder auf die Suche nach Hilfe, insbesondere wenn die Schmerzen wieder sehr stark sind. Sie erzählt aber, dass ihre Suche immer wieder scheitert, weil ihr einfach ,niemand hilft“. Sie berichtet „überall“ gewesen zu sein, als wir sie aber fragen, ob sie Einrichtungen aufgesucht habe, die sich um spezifische Frauenprobleme kümmern, verneint sie das.

L: „Wenn ich davon gehört hätte, wäre ich dahin gegangen. Bei der Frau, wo ich gearbeitet habe, wenn ich sie gefragt habe „wo soll ich mit meinen Schmerzen hingehen?" sie hat mir immer gesagt „es gibt niemanden, du sollst arbeiten. Sonst nehme ich jemand anderen für die Arbeit, eine, die nicht krank ist.", aber dann hätte ich kein Haus gehabt, keinen Platz zu schlafen, also bin ich einfach ruhig geblieben."(I 4, 60-67)

Layla weiß nicht, dass es solche Anlaufstellen gibt, weil ihr bislang die Frage, wohin sie mit ihren Schmerzen gehen könne, mit „es gibt niemanden“ beantwortet wurde. Layla trifft damals aus Angst Wohnung, Arbeitsplatz und Aufenthaltsgenehmigung zu verlieren die Entscheidung, sich diesen Bedingungen zu beugen und erträgt sechs Jahre lang den Schmerz. Ihren Körper zu ignorieren wurde in dieser Zeit eine wichtige Überlebensstrategie, die sie auch nach dem Tod „,der Frau“ nicht ablegt. Für Layla ist die Angst, zurück nach Somalia zu müssen, dominant - dominanter als die Sorge um ihre Gesundheit. 
In einem anderen Teil des Interviews erwähnt Layla, dass sie monatelang auf einen Arzttermin warten müsse und ihr deshalb seit sieben Monaten nichts anderes bliebe, als Schmerztabletten gegen die starken Schmerzen zu nehmen. Layla fühlt sich von ihrem Körper, den Mediziner innen, den Behörden, den Arbeitgeber_innen im Stich gelassen. Trotz all dieser Widrigkeiten kämpft sie dafür, in Italien bleiben zu können. Jeden Tag beginnt der Teufelskreis von Neuem: Ihr Körper signalisiert ihr, krank und arbeitsunfähig zu sein, gleichzeitig bekommt sie immer wieder die Information, arbeiten zu müssen, um in Italien bleiben zu können. Um arbeiten zu gehen, sind ihre Schmerzen aber zu groß. Die Angst, am Ende wieder nach Somalia zurück zu müssen, beherrscht sie, bringt sie dazu, ihre Schmerzen so gut es geht zu betäuben und weiter nach Arbeit zu suchen.

Laylas Interview ist zu entnehmen, wie stark ihr Leben in Italien von Abhängigkeit geprägt ist. Erst war sie abhängig von „,der Frau“, die ihr Arbeit, etwas zu Essen und einen Schlafplatz gegeben hat. Durch die Arbeit bei ,der Frau" war außerdem ihr Aufenthalt in Italien gesichert. Die Bedingung ist allerdings, dass Layla ihre Schmerzen ignoriert, weil „die Frau“ sich sonst eine andere Arbeitskraft sucht. Auch nachdem „die Frau“ gestorben ist, scheint sich der Zustand des Ausgeliefertseins nicht zu verändern: Layla lebt heute in Rom, kann aber aufgrund der Schmerzen keiner Arbeit nachgehen. Da sie der Meinung ist, ihr Leben in Italien sei abhängig von einer Arbeitsstelle, bangt sie um ihre Zukunft in Italien, was sie wiederum dazu veranlasst, ihre Priorität auf die Suche nach Arbeit zu legen und die Schmerzen mithilfe von Schmerzmitteln auszublenden. Dazwischen gibt es Tage, an denen Layla sich auf die Suche nach Hilfe im Umgang mit ihren Schmerzen, doch bleibt sie grundsätzlich abhängig vom Wissen und der Hilfe anderer Menschen.

\subsubsection{Sterilität als größtes Unglück}

Layla gibt an, genital beschnitten zu sein, unter ständigen Infektionen zu leiden und als Jugendliche in Somalia vergewaltig worden zu sein. Auf meine Frage, ob sie denkt, dass ihre Schmerzen mit ihrer Beschneidung zu tun haben könnten, wird sie nachdenklich.

I: „Und ihre Probleme kommen vorwiegend von ihrer Beschneidung?“

L: „Manchmal denke ich, dass das so sein könnte. Ich denke es manchmal. Ich war mal schwanger, es war aber eine Eileiterschwangerschaft und dabei ist ein wichtiges Blutgefäß kaputt gegangen und ich habe ganz viel Blut verloren. Zuhause war das, in Somalia. Es war alles geschwollen, ich hatte so viele Infektionen. Ich wäre gestorben, wenn nicht ein Mann mich gerettet hätte.“

I: „Ein Arzt?“

L: „Ja. Er hat gesagt, innerlich wäre alles kaputt.“ (I 4, 72-79) 
Die Verbindung zwischen Laylas Beschneidung, den wiederkehrenden Infektionen, der Vergewaltigung und ihrer Gebärmutterhalskrebserkrankung scheint für Layla auf den ersten Blick nicht zu bestehen. Nur die Schmerzen sind für sie greifbar, diese erlebt sie jeden Tag deutlich, diese verhindern, dass sie sich eine sichere Existenz in Italien aufbauen kann. Laylas Schlussfolgerung ist daher, sie durch Schmerzmittel zu betäuben. Wenn ihr das gelingt kann sie arbeiten gehen und bekommt ihre Aufenthaltsbewilligung. In diesem Interviewabschnitt zeigt sich Layla aber auch kurz zögerlich: „Manchmal“ denke sie, dass ihre Schmerzen mit der Beschneidung zusammenhängen könnten - „manchmal“. Nach dem kurzen Zögern erwägt sie plötzlich nicht nur ihre Beschneidung, sondern auch ihre Eileiterschwangerschaft als mögliche Ursache für ihre Schmerzen. Das kurze Nachdenken, das sie an dieser Stelle innehalten lässt, könnte Ausdruck dafür sein, dass sie, wenn sie diesem Gedanken Raum lässt, weiß, woher ihre Schmerzen rühren. Da diese Erinnerungen jedoch schmerzhaft sind, versucht Layla sie zu meiden. Aus Angst vor den Gefühlen, die sie mit ihrer Beschneidung, der Vergewaltigung und der Eileiterschwangerschaft verbindet, verdrängt sie die Frage nach der Ursache für den Schmerz - weil die mit den genannten Erlebnissen zu tun haben könnte. Erst in diesem Interviewmoment scheint dieser kurze Augenblick des Nachdenkens dazu zu führen, dass Layla die Erlebnisse in Somalia erinnert. Die Eileiterschwangerschaft, erzählt sie, habe sie aufgrund hohen Blutverlustes nur knapp überlebt. Außerdem habe ihr der sie behandelnde Arzt ihr gesagt, dass sie innerlich zerstört sei, was eine Folge der Vergewaltigung sein kann. ${ }^{17}$ Laylas Strategie im Umgang mit diesen traumatischen Erlebnissen scheint Verdrängung zu sein. Sie verdrängt nicht nur die Erlebnisse, sondern auch ihre Schmerzen, indem sie sie betäubt und den Fokus auf die Arbeitssuche legt.

Als Layla an dieser Stelle weiter erzählen möchte, beginnt sie zu weinen und erzählt, dass ihr sowohl in Somalia als auch Rom medizinisch bestätigt wurde steril zu sein. Genitale Beschneidung, Vergewaltigung, hoher Blutverlust, Schmerzen, Gebärmutterhalskrebs und chronische Schmerzen scheint Layla mithilfe ihrer Mechanismen Verdrängung und Betäubung ertragen zu können, keine Kinder bekommen zu können jedoch nicht.

Wir brechen an dieser Stelle das Interview ab, zum einen weil wir Layla nicht noch tieferen Gefühlen aussetzen wollen, zum anderen aber auch weil wir an diesem Punkt selbst tief betroffen von ihrer Geschichte sind und das Interview mittlerweile mehr Beratungs- als Interviewcharakter hat.

17 Die Vergewaltigung einer infibulierten Frau bringt mit hoher Wahrscheinlichkeit schwere gesundheitliche Folgen mit sich, da dies zum gewaltsamem Aufreißen des Narbengewebes, hohem Blutverlust (von dem sie auch berichtet) und schweren innerlichen Verletzungen mit Infektionsfolge führen kann. 


\subsubsection{Zusammenfassung}

Laylas Geschichte verdeutlicht, wie groß die Angst eines somalischen Flüchtlings ist, zurück nach Somalia geschickt zu werden, und gibt eine Ahnung davon, wie wohl ihr Leben in Somalia ausgesehen haben muss. Trotz all der Widrigkeiten bevorzugt sie ein Leben in Italien. Die Angst, wieder zurück zu müssen, dominiert Laylas Leben und führt dazu, dass sie in ständiger Abhängigkeit bleibt. Gefördert wird die Abhängigkeit durch mehrere Faktoren: Layla trifft in Italien auf ein System, das ihr aus dem eigenen Heimatland nicht bekannt ist, wie beispielsweise ein Gesundheitssystem oder die Möglichkeit, sich Unterstützung bei Beratungsstellen zu holen. Des Weiteren kann sie zwar sehr gut Italienisch sprechen, doch kaum lesen und schreiben, was sie bei ihrer Suche nach Hilfe verstärkt in die Abhängigkeit anderer Personen bringt. Layla kann Informationen nicht noch einmal nachlesen oder Medien (wie Internet, Zeitungen) bei ihrer Suche nach Hilfe nutzen. Auch Hinweisschilder zu bestimmten Ämtern oder Beratungsstellen helfen Layla nur bedingt weiter. Informationen, die sie von anderen bekommt, muss sie sich merken, weil sie sie weder auf Somali noch auf Italienisch aufschreiben kann. Die Suche nach Arbeit oder Hilfe im Umgang mit ihren Schmerzen wird durch diese Faktoren deutlich erschwert, was dazu beiträgt, Layla in eben dieser verzweifelten Lage resignieren zu lassen.

Hinzu kommen Laylas andauernder Schmerz, den sie betäubt, und die damit zusammenhängenden traumatisierenden Erlebnisse, die sie verdrängt. Layla will nur ein sicheres Leben in Italien und eine Arbeitsstelle, doch wird sie immer wieder von der Vergangenheit eingeholt: Weder die Schmerzen noch die damit verbundenen Erlebnisse lassen sich abstellen. Layla sucht verzweifelt nach einem Ausweg, sie sucht nach Menschen, Organisationen, Ärzt innen, die ihr helfen, doch scheint sie niemanden zu finden. Ihre direkte Bereitschaft, mit uns das Interview zu führen, kann auch als Hilferuf interpretiert werden. Layla ist abhängig von anderen, sieht daher keine andere Möglichkeit als von ihrem Leid zu berichten, in der Hoffnung auf jemanden zu treffen, der ihr einen hilfreichen Rat gibt.

\subsection{Saida}

Im Anschluss an das Interview mit Layla folgte ein weiteres mit Saida. Dieses Interview steht stark unter dem Eindruck des vorangegangenen Interviews mit Layla, von dem sowohl die Dolmetscherin als auch ich emotional noch sehr ergriffen sind. Auch das Interview mit Saida findet in einem Café statt. Saida ist schätzungsweise Mitte 30 Jahre alt und hat die ersten 25 Jahre ihres Lebens in einer somalischen Großstadt verbracht. Sie heiratete mit 15 
Jahren und bekam in Somalia zwei Kinder. Sie ist dann ohne ihre Familie nach Saudi-Arabien und von dort aus nach Italien gegangen. In Italien holt sie ihre Familie nach. Saida erzählt nicht, was sie in Saudi-Arabien gemacht hat, auch über ihr Leben in Italien gibt sie lediglich an, noch ein weiteres Kind bekommen zu haben und Hausfrau zu sein. Sie sagt nicht wo sie wohnt, ob ihre Kinder hier zur Schule gehen oder ihr Mann Arbeit gefunden hat. Saida ist sehr wortkarg, beantwortet meist nur die gestellten Fragen, häufig nur mit ,ja“ oder „nein“. Sie erzählte wenig zu sich und erweckt bis zum Ende des Interviews den Anschein, sich nicht wohl zu fühlen.

\subsubsection{Auswirkungen einer den Eingriff problematisierenden Grundhaltung}

Nachdem Saida kurz über ihre Jahre in Somalia berichtet, frage ich sie nach der Rolle der Beschneidung in Somalia.

I: „Kannst du was zur Rolle der Beschneidung in Somalia sagen? Wie war das als du noch dort gelebt hast? Wurde darüber geredet?““

S: „Es war dort normal.“

I: „Wird darüber geredet?"“

S: „Nein.“

I: „Und wie ist das als kleines Kind, wird man da einfach mitgenommen von seiner Mutter oder irgendjemand, ohne das jemand etwas erklärt oder dazu sagt oder wie ist das?"

S: „Ich glaube ich war ganz klein als ich beschnitten wurde.“

I: „Achso, du kannst dich nicht erinnern?“

S: „Nein.“"

I: „Und sie kann sich auch nicht erinnern, dass sie irgendwann einmal mit Freundinnen oder irgendjemandem darüber geredet hat oder mit ihrer Mutter?"

S: „Nein!“

I: „Und als sie geheiratet hat und Kinder bekommen hat, hat sie da Probleme gehabt?"

S: „Nein.“

I: „Sie hat gar keine Probleme gehabt mit ihrer Beschneidung?“

S: „Was willst du wissen? Das ist alles normal! Für mich war das überhaupt kein Problem, die Hochzeit und die Geburt! Naja und bei Geburt, da werdet ihr doch auch geschnitten! Man muss genauso bei euch schneiden, dass das Kind raus kommt!" (I 5, 35-54)

Auf die Frage nach der Rolle der Beschneidung in Somalia berichtet Saida, dass der Eingriff in ihrem Heimatland „normal“ sei. Sie sei im Säuglingsalter beschnitten worden und könne sich daher nicht mehr an den Eingriff erinnern. Saida kennt ihr Genitale demzufolge ausschließlich in beschnittenem 
Zustand, weshalb für sie die Beschreibung, normal' im biologischen Sinne zutreffend ist. Nach diesen ersten Fragen entsteht der Eindruck, als zweifle Saida an der Sinnhaftigkeit dieses Gesprächs. Die Fragen nach „Problemen“, die sie aufgrund ihrer Beschneidung haben soll, blockt sie ab, indem sie mit einem knappen „,nein“ darauf antwortet. Das wiederholte Fragen nach „Problemen“, verärgert sie am Ende und sie fragt: „Was willst du wissen?" Saida scheint sich angegriffen zu fühlen, weil sie wiederholt nach Problemen, die Genitalbeschneidung mit sich bringt, gefragt wird, sie das Gefühl hat, dass wir ihr nicht glauben. Die gestellten Fragen gleichen eher einer Unterstellung, sie lassen Saida keinen Ausweg, weil bereits vorausgesetzt wird, dass Genitalbeschneidung Probleme macht. Diese geschlossene Art zu fragen, bringt Saida in eine Position, aus der ihr nur wenige Reaktionsmöglichkeiten bleiben. Sie fühlt sich in die Ecke gedrängt und weigert sich, die Position des ,Opfers ${ }^{6}$ ihrer Traditionen einzunehmen. Stattdessen fragt sie verärgert, was ich von ihr wissen wolle. Vermutlich wird Saida nicht das erste Mal damit konfrontiert, dass Menschen europäischer Herkunft weibliche Beschneidung problematisieren, jetzt nutzt sie die Gelegenheit, um deutlich zu machen, dass sie keine Probleme mit ihrer Beschneidung hat und sich auch nicht einreden lässt, welche haben zu müssen.

Am Ende sagt sie: „Naja und bei Geburt, da werdet ihr doch auch geschnitten! Man muss genauso bei euch schneiden, dass das Kind raus kommt!“ Mit diesem letzten Satz deutet Saida an, dass sie vermutlich weiß, worauf die problematisierenden Fragen abzielen. Sie ist aber nicht bereit, sich auf diese Perspektive einzulassen. Saida unterstreicht stattdessen, keine Probleme zu haben und auch bei der Entbindung nicht mehr geschnitten worden zu sein als unbeschnittene Frauen. Es klingt, als wolle sie deutlich sagen, dass sie als infibulierte Frau so ,normal' ist wie unbeschnittene Frauen. Des Weiteren zeigt Saida mit dieser Äußerung auch, dass ihr die Unterschiede zwischen beschnittenem und unbeschnittenem Genitale bewusst sind. Das steht jedoch im Gegensatz zu ihrer Aussage, nicht zu wissen, was bei Beschneidung gemacht werde. Wahrscheinlicher scheint jetzt aber, dass sie die Situation, sich für ihre ,Tradition' rechtfertigen zu müssen, schon erlebt hat und sich zum Zeitpunkt des Interviews in diese oder ähnliche Situationen zurück versetzt fühlt. Möglicherweise wählt sie deshalb die Strategie, anzugeben nicht detailliert über Beschneidung Bescheid zu wissen, um nicht unter Rechtfertigungsdruck zu geraten und der Gefahr zu entgehen, sich durch uns in eine unterlegene Position bringen zu lassen, nämlich die des Opfers ihrer ,Traditionen'.

Als wolle Saida zeigen, dass sie wirklich keinerlei negative Konsequenzen von ihrer Beschneidung davon getragen hat, erzählt sie im Anschluss, dass sie vorhabe ihre Tochter beschneiden zu lassen, sofern sie bei der Rückkehr nach Somalia nicht schon zu alt dafür sei. Zum Zeitpunkt des Interviews war ihre Tochter vier Jahre alt. Warum sie im Hinblick auf die Beschneidung 
ihrer Tochter eine Altersgrenze festlegt, begründet Saida nicht. Eine Lesart dafür könnte sein, dass sie davon ausgeht, die Rückkehr nach Somalia könne sich noch einige Jahre hinauszögern und ihre Tochter dann bereits verheiratet sein. Findet ihre Tochter unbeschnitten einen Mann, wäre die Beschneidung nach der Hochzeit hinfällig. Saida weiß, dass der Eingriff in Italien verboten ist, deshalb wolle sie warten bis sie wieder in Somalia sind.

In diesem Zusammenhang kommt die Frage auf, ob sie schon einmal davon gehört habe, dass somalische Familien ihre Töchter in Italien beschneiden lassen. Sie verneint die Frage, weil die somalischen Familien wüssten, dass der Eingriff in Italien verboten sei.

S: „Ich weiß nicht, warum es verboten ist. Aber ich wüsste auch nicht wo ich das heimlich machen könnte. Wenn ich aber mal zurück kehre nach Somalia und meine Tochter noch jung ist, dann mache ich es."

I: „Ja, klar, weil du der Meinung bist, dass es gut ist für deine Tochter.“

$\mathrm{S}$ : „Ja. Man hat mir das doch auch angetan, das muss ich für sie auch machen, das ist unsere Tradition."

I: ,"Angetan“" klingt wie „das war nicht schön“, aber trotzdem will sie das ihrer Tochter auch antun?"

S: „Ich meinte, man hat mich auch beschnitten und ich mache das, weil es nun mal unsere Tradition ist.“ (I 5, 77-86)

Saida weiß um das Verbot, gibt aber gleichzeitig an, nicht zu wissen weshalb ein solches existiert. Aus Gesagtem ist anzunehmen, dass Saida sich nicht strafbar machen möchte, vielleicht aus Angst, Italien sonst verlassen zu müssen. Hinzu kommt aber auch, dass sie angibt, nicht zu wissen wo sie ihre Tochter beschneiden lassen könnte. Saida hinterlässt den Eindruck, mit der Situation, ihre Tochter momentan nicht beschneiden lassen zu können, unzufrieden zu sein. Daher stellt sich die Frage, inwieweit die gesetzliche Regelung sie daran hindern würde, würde sie von der Möglichkeit erfahren, ihre Tochter auch in Italien beschneiden lassen zu können. Momentan wählt sie den Ausweg aus dieser für sie nur schwer zu akzeptierenden Situation, in dem sie darauf hofft, möglichst bald wieder zurück ins Heimatland gehen zu können, um den Eingriff dort vornehmen zu lassen.

Bezüglich ihrer Wortwahl „man hat mir das auch angetan“ bleibt fraglich, ob sich Saida tatsächlich auf diese Weise geäußert hat. Es ist auch möglich, dass sie nicht diesen negativ konnotierten Ausdruck ausgesucht hat, sondern die Dolmetscherin diese Redewendung gebraucht hat, da in diesem Interview immer wieder zwischen Somali und Italienisch hin- und hergewechselt wurde. Saida gibt sich auch in diesem Interviewteil zurückhaltend, beantwortet lediglich die gestellten Fragen. Als sie davon spricht, ihre Tochter in Somalia beschneiden lassen zu wollen, lacht sie. Sie lacht als wolle sie zeigen, dass sie es zwar ein wenig lächerlich findet, mit der Beschneidung noch warten zu müssen, dies aber hinnehmen würde. Möglich ist auch, dass sie lacht, um sich 
noch einmal deutlich von uns und unserer ablehnenden Haltung gegen weibliche Beschneidung abzugrenzen.

\subsubsection{Zusammenfassung}

Saidas ablehnende teilweise angriffslustige Haltung kann als Reaktion auf meine sehr direktiven, wenig Spielraum eröffnenden Fragen interpretiert werden. Noch deutlich unter dem Eindruck des vorangegangenen Interviews mit Layla, wird Genitalbeschneidung vom ersten Satz an problematisiert. Saida hat keine andere Wahl, als sich dieser Problematisierung anzuschließen oder sich deutlich davon abzuwenden - und sie entscheidet sich für letztere Variante. Immer wieder entsteht der Eindruck, dass Saida ganz bewusst die Strategie wählt, zu betonen keine Probleme mit Beschneidung zu haben und auch nicht genau zu wissen, welche Probleme Beschneidung überhaupt mit sich bringen kann. Auch wenn an manchen Stellen deutlich wird, dass Saida nicht so unwissend ist, bleibt sie bei der Betonung ihrer Ahnungslosigkeit. Sie räumt zwar ein, davon gehört zu haben, dass Genitalbeschneidung in Italien verboten sei, doch wisse sie nicht genau warum. Wir als interviewende Personen scheinen bei Saida Ablehnung auszulösen, weil sie in uns zwei Frauen sieht, die die Beschneidung verurteilen und sie als ,Opfer" stigmatisieren. Um sich von uns und unserer Darstellung von beschnittenen Frauen abzugrenzen, beantwortet sie nur sehr einsilbig die gestellten Fragen und zieht sich zurück. Es entsteht eine Mauer zwischen uns, die ohne die generelle Problematisierung von Genitalbeschneidung vermutlich nicht entstanden wäre. Das Interview zeigt, wie sensibel Gespräche mit Betroffenen ablaufen müssen, um nicht - wie hier geschehen - eine unüberwindbare Distanz aufzubauen und den Kontakt zu den Frauen zu verlieren. 


\subsection{Zentrale Ergebnisse der Fallanalysen}

Die Analyse der qualitativen Interviews mit den somalischen Frauen hat ergeben, dass die Frauen ganz unterschiedlich mit ihrer eigenen Beschneidung, der Bewertung der Praktik und den Konsequenzen derselben umgehen. Trotz des unterschiedlichen Umgangs sind einige Gemeinsamkeiten auszumachen, die nachfolgend genannt und im Anschluss erläutert werden. Die Rekonstruktion der Interviews hat gezeigt, dass

- Mädchenbeschneidung in Somalia als ,normal ‘ beschrieben wird.

- die Migration in ein Genitalbeschneidung ablehnendes Land ein Einschnitt im Leben der interviewten Frauen ist, der sowohl negative als auch positive Veränderungen mit sich bringen kann.

- die interviewten Frauen in Einwanderungsland negative Erfahrungen im Umgang mit ihren beschnittenen Genitalien gemacht haben.

- die spezifische Situation von somalischen, geflohenen Frauen zu wenig Berücksichtigung findet.

Die einzelnen Ergebnisse werden nachfolgend präzisiert.

\subsubsection{Mädchenbescheidung wird als gesellschaftlich etablierte Norm in Somalia beschrieben}

Aus den Interviews geht deutlich hervor, dass weibliche Genitalbeschneidung im Heimatland der Frauen als eine unhinterfragte Norm dargestellt wird, was sich auch daran zeigt, dass in Somalia noch 98\% aller Mädchen und Frauen beschnitten sind (WHO 2008, S. 208). Zur Begründung des Eingriffs nennen die befragten Frauen Argumente wie weibliche Genitalbeschneidung diene zur Sicherung der Jungfräulichkeit und wäre zur Aufnahme in die soziale Gemeinschaft notwendig. Besondere Bedeutung habe die Infibulationsnarbe, da diese dem künftigen Ehemann als Beweis für die Jungfräulichkeit seiner Braut diene. Dies sei laut den interviewten Frauen auch der Grund, weshalb Männer nur eine infibulierte Frau heiraten wollten. Sara wünscht sich deshalb eine Gesellschaft - worunter sie vor allem somalische Männer fasst -, die Frauen mit unbeschnittenen Genitalien akzeptiert, ohne diesen zu unterstellen, sexuell ausschweifend zu sein. Folglich werden unbeschnittene Frau mit Attributen wie ,sexuell ausschweifend' oder , untreu“ belegt, während beschnittene als ,jungfräulich` und ,treu' gelten. Diese Konstruktionen tragen zum Erhalt der Praktik bei und zwingen Frauen zur Genitalbeschneidung. Gleichzeitig berichten die interviewten Frauen aber, dass es nicht die Männer seien, die den Eingriff für die Töchter organisieren und durchführen würden, diese Aufgabe käme den Frauen zu. Vergleichbar mit 
der von Spivak beschriebenen Witwenverbrennung (Castro Varela/Dhawan 2005, S. 59) ordnen sich Frauen den patriarchalen Strukturen unter, die sie zu Hüterinnen der ,Tradition“ machen und auf diese Weise für den Erhalt dieser unterdrückenden Strukturen sorgen. Entscheidet sich eine Frau dafür, ihre Tochter unbeschnitten zu lassen, droht der soziale Ausschluss. Der Druck der Gemeinschaft ist enorm, wie am Beispiel Sara deutlich zu sehen ist. Bereits junge Mädchen üben Druck auf unbeschnittene Gleichaltrige aus, indem sie sie mit der Begründung, ein ,schlechtes' Mädchen zu sein, aus ihrer Gemeinschaft ausschließen und beschimpfen. Der Wunsch des damals zehnjährigen Mädchens, wieder Zugehörige der sozialen Gemeinschaft zu sein, ist stärker als die Warnung ihrer Mutter vor weiblicher Beschneidung. Der Druck der Mädchen treibt Sara dazu, sich gegen den Willen ihrer Mutter durchzusetzen und selbst für ihre Beschneidung zu sorgen. Die im Rahmen Asefaws (2008) durchgeführten Interviews mit eritreischen Frauen haben ebenfalls ergeben, dass die Mädchen und Frauen unter hohem sozialen Druck stehen, der dazu führt, dass sie sich dem Eingriff aussetzen und ihn als Teil ihrer weiblichen Identität begreifen (Asefaw 2008, S. 41 ff.). Die befragten Interviewpartner_innen benennen weibliche Genitalbeschneidung als eine Praktik, die seit vielen Jahrhunderten eine gesellschaftlich etablierte Norm in Somalia darstellt. Diesen Aussagen zufolge steht sie für soziale Zugehörigkeit und wird als ein bedeutender Teil der weiblichen Identität konstruiert.

\subsection{Migration wird sowohl als Einschnitt als auch Möglichkeit des Perspektivwechsels erlebt}

Die interviewten Frauen erzählen, dass sie bis zum Zeitpunkt der Einwanderung in ein europäisches Land mit beschnittenen Genitalien gelebt und diese als ,normal' betrachtet haben. Erst nachdem sie im Einwanderungsland mit Ärzt innen oder Hebammen konfrontiert wurden, die schockiert oder unwissend auf ihre beschnittenen Genitalien reagiert haben, wurde den Frauen bewusst, dass sie nicht länger der Norm entsprachen. Die Auseinandersetzung mit der Tatsache, nicht länger der Norm zu entsprechen, kombiniert mit den teilweise negativen Reaktionen von Vertreter_innen der Mehrheitsgesellschaft des jeweiligen Einwanderungslands auf ihre beschnittenen Genitalien, führte bei den interviewten Frauen zu ganz unterschiedlichen Umgangsstrategien.

Mit der Einwanderung nach Deutschland wird für Sara ,normal' zu ,unnormal'. Die Infibulationsnaht, ursprünglich Zeichen ihrer ,Reinheit' und ,Weiblichkeit', wird im Einwanderungsland zu einer, Verstümmelung ${ }^{6}$. Sara beginnt den Eingriff zu hinterfragen, stellt sich die Sinn- und Schuldfrage. Dieses Hinterfragen geht mit tiefem Bedauern über die damals getroffene 
Entscheidung und der Enttäuschung einher, von der eigenen Mutter nicht ausreichend beschützt worden zu sein. In den wiederkehrenden Vergleichen ihres Lebens mit dem ,europäischer' Frauen oder dem Aufwachsen und dem Leben ihrer eigenen Tochter in Deutschland sieht Sara vor allem, mit wie vielen Einschränkungen der Eingriff verbunden ist. Saras Ambivalenz, die sich besonders in der wiederkehrenden Schuldfrage zeigt, erinnert an die von Strenge als „Betrayal-Trauma“ (Strenge 2013, S. 328) bezeichnete psychische Folge weiblicher Genitalbeschneidung. Folgt man den Ausführungen Strenges, wird ein solches Trauma durch die Beschneidung in der Kindheit ausgelöst, da die Mädchen mit diesem Eingriff einen tiefen Bindungsverlust zu den Eltern erleben. Die Erfahrung, von den primären Schutzpersonen nicht beschützt worden zu sein, traumatisiert die Mädchen - ein Trauma, das sich in der wiederkehrenden Schuldfrage äußern kann. Aufgrund der Tatsache, dass Eltern den Mädchen aber sagen, dass der Eingriff gemacht werden müsse, um sie zu ,reinen' Frauen zu machen, geben sich die Mädchen selbst die Schuld, schließlich sind sie es, die fehlerhaft gewesen seien und deshalb beschnitten werden mussten. Ein Kreislauf, der sich im Interview Saras deutlich zeigt: Sara ist sich bis zum Ende des Interviews nicht sicher, ob nun ihre Mutter, die Gesellschaft oder doch sie selbst Schuld am Eingriff haben - eine Frage, die sie wiederkehrend beschäftigt. Hinzu kommt, dass immigrierte Frauen den gesellschaftlichen Kontext, in dem der Eingriff seinen Bedeutung hatte, verlassen haben und im Einwanderungsland $\mathrm{zu}$ dem Bewusstsein kommen, durch den Eingriff einen Teil ihres Körpers verloren zu haben. Diese Bewusstwerdung kann laut Strenge zu einer Retraumatisierung, einem zweiten „Betrayal-Trauma“ führen (Strenge 2013, S. 332), da der Eingriff im Einwanderungsland seine Bedeutung verliert. Die eingewanderten Frauen fühlen sich betrogen, da ihnen bewusst wird, dass sie durch den Eingriff nichts gewonnen, sondern im Gegenteil, Vieles verloren haben. Auch Asefaw (2008) hält als ein Ergebnis ihrer Studie fest, dass die interviewten eritreischen Frauen, die ihr Heimatland verlassen haben, psychisch mehr leiden als die, die in Eritrea geblieben sind (Asefaw 2008, S. 69). Asefaw erklärt dieses Phänomen damit, dass für beschnittene Frauen, die ihren gesellschaftlichen Kontext verlassen, die „Sinn gebenden Faktoren der weiblichen Beschneidung, wie beispielsweise soziale Identität und gesellschaftliche Akzeptanz“" (Asefaw 2008, S. 69) weg fallen würden. In der Migration würde den Frauen stattdessen „Mitleid und Empörung“ (ebd.) entgegengebracht, was dazu führe, dass sie sich nicht nur sozial ausgegrenzt fühlten, sondern auch ein Bewusstsein dafür entwickelten, dass ihnen und ihrem Körper Gewalt angetan wurde. Das würde dazu führen, dass beschnittene Migrantinnen mehr unter den psychischen Konsequenzen des Eingriffs leiden würden. Fraglich bleibt, ob die psychischen Folgen tatsächlich erst nach dem Verlassen des gesellschaftlichen Kontextes auftreten oder ob die Frauen nicht schon im Heimatland unter psychischen Folgen leiden, diese aber nicht diagnostiziert werden. 
In Saras Fall zeigen die wiederkehrende Schuldfrage und das tiefe Bedauern über ihre vermeintliche Entscheidung für den Eingriff, dass Sara von einer psychologischen Begleitung im Umgang mit dem erlittenen Trauma profitieren könnte.

Des Weiteren zeigen die im Rahmen dieser Forschungsarbeit durchgeführten Interviews, dass noch immerzu wenige Gynäkolog_innen und Hebammen existieren, die im Umgang mit infibulierten Frauen informiert sind. Die Interviewpartnerinnen berichten entweder von Gynäkolog_innen untersucht worden zu sein, die sich von ihren Genitalien schockiert gezeigt hätten, oder von Hebammen, die ihnen während des Geburtsverlaufs nicht professionell zur Seite stehen konnten, da sie zu wenig Informationen im Umgang mit infibulierten, gebärenden Frauen hatten. Ergebnisse, die sich auch mit den Forschungen Asefaws (2008) und Graf (2012) decken (Asefaw 2008, S. 74; Graf, S.147). Die Ergebnisse Grafs, in Kombination mit den Erfahrungen der interviewten Eritreerinnen und Somalierinnen zeigen, dass medizinische Fachkräfte noch immer zu wenig über den Umgang mit beschnittenen Frauen informiert sind. Beschnittene Frauen können sich in Deutschland demnach nicht darauf verlassen, professionell medizinisch versorgt zu werden. Dies beinhaltet nicht allein die Behandlung möglicher physischer Konsequenzen, aus Sicht der interviewten Frauen sollte die medizinische Begleitung außerdem mit mehr Informationen zu den Konsequenzen weiblicher Genitalbeschneidung verbunden sein. Ebenso wünschen sich die Interviewpartnerinnen, dass mehr somalische Frauen in die Beratung und Begleitung eingebunden werden, da es dann möglich wäre, die Gespräche auf Somali durchzuführen. Dies würde außerdem den weiteren Vorteil mit sich bringen, dass keine Familie aufgrund mangelnder Sprachkenntnisse von Informationen ausgeschlossen bliebe. Ein weiterer wichtiger Aspekt ist, dass der Umgang mit beschnittenen Frauen von einer angemessenen Haltung geprägt sein sollte. Möchte man erreichen, dass beschnittene Frauen die Informationen annehmen und die Notwendigkeit des Eingriffs hinterfragen, ist ein offener und wertschätzender Kontakt besonders wichtig.

Die Interviews mit den befragten somalischen Frauen zeigen jedoch auch, dass es Frauen gibt, die angeben keine Probleme mit ihren beschnittenen Genitalien zu haben. So benennt Saida zwar während der Geburt ihrer Kinder geschnitten worden zu sein, doch, betont sie, handle es sich dabei um einen Vorgang, den auch unbeschnittene Frauen durchlaufen müssten. Die Problematisierung weiblicher Genitalbeschneidung ist aus ihrer Sicht nicht notwendig. Eine solche Reaktion kann als Schutzbehauptung interpretiert werden, um sich von der ,westlich ' geprägten, stigmatisierenden Vorstellung des ,hilflosen Opfers' abzugrenzen. Es ist jedoch auch möglich, dass infibulierte Frauen nicht zwingend unter negativen Konsequenzen des Eingriffs leiden. Ebenso möglich ist, dass gesundheitliche Schwierigkeiten nicht mit der Beschneidung in Verbindung gebracht werden, so wie beispielsweise 
Layla äußert, sich nicht sicher zu sein, ob der Eingriff tatsächlich mit ihren gesundheitlichen Schwierigkeiten zusammenhängt. Sie berichtet zwar während des gesamten Interviews von gesundheitlichen Problemen, zeigt sich am Ende des Interviews aber unsicher bezüglich der Frage, ob sie eine Verbindung zu Genitalbeschneidung sehen würde. Dies zeigt, wie auch in den beiden bereits genannten Studien von Asefaw (2008) und Behrendt (2004), dass Unwissenheit über den Eingriff und dessen Folgen besteht und medizinische Konsequenzen nicht zwingend mit dem Eingriff in Verbindung gebracht werden. Bei Layla scheint jedoch hinzuzukommen, dass sie versucht, ihre Schmerzen beziehungsweise die damit zusammenhängenden Ereignisse, wie ihre Beschneidung und die Vergewaltigung, zu verdrängen. Sie spricht von Arbeit, an die der sichere Aufenthalt in Italien geknüpft sei und dass all das immer wieder von ihren starken Schmerzen durchkreuzt würde, die sie aber mit Schmerzmittel versucht zu betäuben. Erst am Ende des Interviews brechen die mit den genannten Erlebnissen verbundenen Emotionen heraus und sie beginnt zu weinen. Laylas Verdrängungsmechanismus, den Strenge ebenso als einen möglichen Umgang mit der erlittenen Traumatisierung nennt (Strenge 2013, S. 328), scheint Layla so lange vor der Erinnerung zu schützen, wie sie nicht von den Erlebnissen berichten muss. Während der Erzählung scheinen die verdrängten Erlebnisse jedoch wieder präsent zu werden und Layla emotional sehr aufzuwühlen. Laylas Geschichte verdeutlicht auch, mit wie vielen Schwierigkeiten das Leben eines Flüchtlings verbunden ist. In ihrem Interview schildert Layla ein Leben voller Gewalt, Verzweiflung und Angst, das sich auch der Einwanderung nach Italien nicht zum Besseren wendet. In Italien fürchtet sie jeden Tag, wieder zurück nach Somalia geschickt zu werden, obwohl sie täglich für einen gesicherten Aufenthalt in Italien kämpft. Dennoch fehlen Layla sowohl finanzielle Mittel als auch eine adäquate medizinische Versorgung und Begleitung, um ihr Überleben in Italien zu sichern. Berichte zu der Situation von Flüchtlingen in Italien zeigen, dass Laylas Situation beispielhaft für die vieler Flüchtlinge in Italien ist. Flüchtlinge oder Asylsuchende wohnen in beschädigten Häusern und leben häufig in Armut, da sie keine staatliche Unterstützung erhalten und kaum eine Chance auf dem italienischen Arbeitsmarkt haben (Schwarz 2013). Hinzu kommt, dass Layla eine infibulierte Frau ist, die unter schweren medizinischen Konsequenzen des Eingriffs leidet. Ihr Beispiel zeigt, dass die spezifische Situation, in der sich beschnittene, asylsuchende Frauen befinden, im Asylverfahren und der Beratung und Begleitung von Flüchtlingen und Asylsuchenden zu wenig berücksichtigt wird. Die gesundheitliche Situation, in der Layla aufgrund der ihr widerfahrenen geschlechtsspezifischen Gewalt (weibliche Genitalbeschneidung und Vergewaltigung) ist, scheint entweder nicht als Asylgrund zu zählen oder Frauen werden nicht darüber informiert, dass geschlechtsspezifische Fluchtgründe Asylgrund sein können. Layla geht jedenfalls davon aus, dass sie nur in Italien bleiben kann, wenn sie eine Ar- 
beitsstelle findet. Ein Beispiel, das verdeutlicht, dass Frauen im Einwanderungsland Italien keine oder eine nur unzureichende Begleitung und Beratung in Asyl- und Bleiberechtsfragen erhalten. Außerdem zeigt sich an Laylas Beispiel die Überlagerungen gesellschaftlichen Macht- und Herrschaftsverhältnissen besonders deutlich. In ihrer von Diskriminierung geprägten Situation reicht es nicht aus, die einzelnen Kategorien (wie beispielsweise Nation oder Geschlecht) einzeln zu betrachten und für das Zustandekommen ihrer schwierigen sozialen Situation zu analysieren. Laylas Situation ist aus intersektionaler Perspektive zu betrachten, was bedeutet, dass die einzelnen Kategorien in ,ihrer ,Verwobenheit“ oder ,Überkreuzung““" (Walgenbach 2012, S.81) wahrzunehmen und zu analysieren sind. Die restriktiven Gesetze der Asylpolitik spiegeln gesellschaftliche Macht- und Herrschaftsverhältnisse wider, deren Auswirkungen Layla spürt und benennt. Hinzu kommen die Folgen der körperlichen Gewalt, die Layla erfahren hat, die aber im Rahmen der Gesetzgebung keine Rolle zu spielen scheinen. Doch hindern diese sie daran, einer Arbeit nachzugehen und sich - zumindest ein Stück - aus den diskriminierenden Strukturen zu lösen. Arbeitslos zu sein, wirkt sich auf ihren Aufenthaltsstatus und ihre Wohnsituation aus. Beides, so berichtet Layla, ist wiederum an die gesundheitliche Versorgung gekoppelt. Der tägliche Kampf, neue Wege aus der nahezu ausweglosen Lage zu finden, sorgt für Laylas Verzweiflung und Trauer.

Im Gegensatz zu Layla oder Saida, steht Ayaan. Sie lebt seit vielen Jahren in England und ist sich der gesundheitlichen Konsequenzen einer Infibulation bewusst. Als langjährig tätige Krankenschwester kennt sie die Auswirkungen des Eingriffs sowohl aus beruflicher als auch aus eigener Erfahrung. Sie benennt die Schwierigkeiten detailliert und geht gleichzeitig darauf ein, wie ein professioneller medizinischer Umgang mit infibulierten Frauen auszusehen hat. Sie betrachtet den Umgang mit infibulierten Frauen sehr pragmatisch, beschränkt ihn auf die physischen Folgen und die notwendigen medizinischen Interventionen. Im Vordergrund des Umgangs mit weiblicher Genitalbeschneidung steht für sie daher allein der medizinische Umgang mit infibulierten Frauen - die Bewertung einer in Somalia etablierten Norm spielt vor diesem Hintergrund eine untergeordnete Rolle, so wie auch ihr persönliches Erleben bezüglich der am eigenen Körper erfahrenen Konsequenzen der Infibulation.

Nadfia dagegen geht wenig auf die medizinischen Folgen des Eingriffs ein. Sie wünscht sich, dass beschnittene Frauen in Zukunft so ,normal' wie Unbeschnittene behandelt werden und meint damit nicht alleine den Umgang mit den aus dem Eingriff resultierenden Folgen. Ihr geht es im Besonderen um die Bewertung einer gesellschaftlich etablierten Norm, die je nach Perspektive unterschiedlich ausfällt. Nadifa ärgert sich über den ,europäischen' Blick auf weibliche Genitalbeschneidung, da sich dieser durch ,westliche“ Arroganz auszeichne und davon geprägt sei, die Überlegenheit ,europäischer" 
Normen hervorzuheben. Dieses Verhalten erinnert Nadifa an Kolonialzeiten, in denen Somalis, wie sie sagt, ,zu Gast im eigenen Land“ (Interview Nadifa, Zeile 105) gewesen seien. Das Interview mit Nadifa spiegelt einige Erkenntnisse postkolonialer Studien und Kritischer Weißseinsforschung wider. So ärgert sie sich einerseits über den ,europäischen' Blick auf ihr Heimatland Somalia, der davon geprägt sei, aus den Somalis eine homogene Einheit zu konstruieren und diese dann im Vergleich zum vermeintlich ,zivilisierten Europa herabzustufen, ein Prozess, der sich durch Macht- und Herrschaftsverhältnissen auszeichnet (Castro Varela/Dhawan 2005, S. 34) und zur Rechtfertigung der Kolonisierung bestimmter Bevölkerungsgruppen gedient hat und noch bis heute dient. Andererseits sagt sie, dass auch der Diskurs über weibliche Genitalbeschneidung in der Schweiz und der daraus resultierende Umgang mit beschnittenen Frauen von einer herabwürdigenden Haltung geprägt seien. Folgt man in diesem Zusammenhang der Kritischen Weißseinsforschung, empfindet Nadifa den, wie sie es bezeichnet, ,europäischen' Umgang mit beschnittenen somalischen Frauen herabwürdigend, weil auch dieser mit einem Machtgefälle einhergehe. Die Konstruktion weißer als ,natürlich' überlegene Norm und die damit verbundene Vorstellung, Schwarze seien nach weißem Vorbild zu „erziehen und zivilisieren“ (Arndt 2005, S. 26), bringt eine Herabstufung Schwarzer Menschen mit sich, von der aus Nadifas Sicht der ,europäische' Umgang mit somalischen Frauen geprägt ist. Frauen wie Nadifa vertreten die Meinung, dass die Arbeit gegen weibliche Genitalbeschneidung von Betroffenen solange nicht angenommen werde, wie sie das Gefühl von Unterlegenheit vermittelt bekommen würden - Beschnittene ,normal' $\mathrm{zu}$ behandeln, heißt aus ihrer Sicht, die Herabwürdigung somalischer Normen abzulegen und aufzuhören Somalis nach ,europäischem Vorbild erziehen zu wollen.

Trotz der genannten Schwierigkeiten, die sich mit der Migration in ein Genitalbeschneidung ablehnendes Einwanderungsland für beschnittene Frauen ergeben können, verdeutlichen die Interviews auch, dass das Leben im Einwanderungsland Chancen birgt. Die Einwanderung bietet den Frauen die Möglichkeit, den Eingriff erstmals kritisch zu hinterfragen und sich bei ihren eigenen Töchtern dagegen zu entscheiden. In Somalia dagegen wäre eine solche Entscheidung nahezu unmöglich, zumal der Eingriff als solcher meist unhinterfragt tradiert wird, so erzählen die Interviewpartnerinnen. Einzelne Personen, wie beispielsweise Saras Mutter, hätten daher kaum eine Chance, ihre Töchter vor dem Eingriff zu schützen. Im Einwanderungsland dagegen besteht die Möglichkeit, sich über die physischen und psychischen Konsequenzen zu informieren. Die Informationen, gegebenenfalls kombiniert mit Beratungsgesprächen, bieten den Frauen und ihren Familien die Gelegenheit, den Eingriff im Hinblick auf mögliche Konsequenzen - dies betrifft sowohl die physischen und psychischen als auch strafrechtlichen Konsequenzen noch einmal neu zu bewerten. Im Fall der Interviewpartnerinnen, die bereits 
Töchter hatten, zeigt sich, dass sich alle dafür entschieden haben, den Eingriff nicht durchführen zu lassen. Während die Mehrheit der Frauen aufgrund der negativen Folgen, die der Eingriff für ihre Töchter haben kann, zu dieser Entscheidung gekommen sind, hat eine Interviewpartnerin aus Angst vor strafrechtlichen Konsequenzen entschieden, den Eingriff solange wie sie sich im Einwanderungsland aufhält nicht durchführen zu lassen. Die Aussagen der Frauen verdeutlichen, dass die Migration in ein weibliche Genitalbeschneidung ablehnendes Einwanderungsland für Mädchen bedeuten kann, unbeschnitten zu bleiben.

Abschließend kann festgehalten werden, dass die Analyse der Interviews ergeben hat, dass die Einwanderung in ein weibliche Genitalbeschneidung ablehnendes Land, erstmals ein Hinterfragen des Eingriffs möglich macht. Welche Prozesse durch die Erfahrungen im jeweiligen Einwanderungsland in den befragten Frauen angeschoben werden, sind so individuell wie ihre Erfahrungen selbst. Die Interviews zeigen jedenfalls, dass das Hinterfragen und Diskutieren weiblicher Genitalbeschneidung dazu führen kann, dass die im Einwanderungsland geborenen und aufwachsenden Töchter unbeschnitten bleiben. Im Umgang mit ihrer eigenen Beschneidung weisen die befragten Frauen allerdings darauf hin, dass noch viel uninformiertes medizinisches Fachpersonal existiert. Unwissenheit, insbesondere in Bezug auf infibulierte Frauen, hat negative Folgen auf deren medizinische Beratung und Begleitung. Neben den medizinischen Konsequenzen, die diese Unwissenheit mit sich bringen kann (wie Notfallsituationen oder nicht erkannte chronische Folgen), berichten die befragten Frauen von schockierten Ärzt_innen und Hebammen. Reaktionen dieser Art sorgen bei beschnittenen Frauen für Unsicherheit und Scham, was wiederum keine Basis für ein Vertrauensverhältnis darstellt und zur Folge haben kann, dass betroffene Frauen medizinische Untersuchungen nicht mehr wahrnehmen. Ferner zeigen die Interviews, dass Frauen von psychischen Konsequenzen betroffen sein können. Psychologische beziehungsweise psychotherapeutische Begleitung bislang jedoch nicht angeboten oder in Anspruch genommen worden zu sein scheint. Abschließend soll noch einmal auf die genannte Haltung eingegangen werden, die teilweise als herabwürdigend wahrgenommen wurde. Im Umgang mit betroffenen Frauen ist von Bedeutung, ihnen auf Augenhöhe zu begegnen, sie in ihren Bedürfnissen wahr- und ernst zunehmen. Druck oder die Androhung von Sanktionen, Belehrungen werden als destruktiv benannt. Aufklärungsund Beratungsarbeit zu den Folgen und den gesetzlichen Regelungen weiblicher Genitalbeschneidung im Einwanderungsland dagegen, werden als sinnvoll und wichtig erachtet. 


\section{Ergebnisse der migrierten Männer somalischer Herkunft}

Bevor die zentralen Aussagen der männlichen Interviewpartner zum Thema weibliche Genitalbeschneidung präsentiert werden, wird nachfolgend in Kürze auf ihre Person und ihren Lebensweg eingegangen.

In Deutschland wurden zwei Männer zum Thema Mädchenbeschneidung interviewt. Der erste Interviewpartner, Feysal, ist 43 Jahre alt wurde in Somalia geboren, wuchs dort auf und machte in Somalia sein Abitur. Danach begann er zu studieren, konnte jedoch das Studium aufgrund des Krieges in Somalia nicht beenden. Gemeinsam mit seiner Frau flohen sie nach Deutschland, wo sie mittlerweile seit vielen Jahren gemeinsam mit ihren Kindern leben.

Jamaal, 52 Jahre, ist in Äthiopien geboren und hat 17 Jahre als Nomade in der Grenzregion zwischen Somalia und Äthiopien gelebt. Als junger Erwachsener ging er in eine kleine somalische Stadt und machte eine Ausbildung zum Krankenpfleger. 1983 kam er nach Deutschland, da er medizinische Hilfe benötigte. Als deutlich wurde, dass sich die politische Situation in Somalia verschärfte, bat er um Asyl. Er lebt seitdem mit seiner ebenfalls aus Somalia stammenden Frau und den gemeinsamen Kindern in einer deutschen Großstadt.

Abshir, der zum Zeitpunt des Interviews 38 Jahre alt, wurde in einer großen somalischen Stadt geboren und aufgewachsen. Nach dem Abitur verließ er seine Heimat, da er sonst zum Militärdienst gezwungen worden wäre und die politische Lage in Somalia zu diesem Zeitpunkt bereits instabil war. Er floh erst nach Kenia, dann nach Italien und immigrierte 1988 schließlich in die Schweiz. Mittlerweile hat er den schweizerischen Pass und lebt seit vielen Jahren gemeinsam mit seiner Frau und den gemeinsamen Kindern in einer schweizerischen Stadt. Abshir ist ehrenamtlich sehr engagiert und hat lange Zeit in Projekten gegen Mädchenbeschneidung gearbeitet.

Cigal ist 48 Jahre alt und lebt seit 1989 in England. Auch er wuchs in Somalia auf, wo er eine Zeit die Schule besuchte. Der Vater, der für eine britische Ölfirma arbeitete, entschied damals, mit der ganzen Familie nach England auszuwandern. So konnten seine Mutter, er und seine 12 Geschwister Somalia noch vor Beginn des Krieges verlassen. Cigal bezeichnet sich heute als englischen Somali.

Nabiil ist 32 Jahre alt und in Somalia geboren. Er hat dort gelebt bis er 18 Jahre alt war und dort Abitur gemacht. Als Einziger von zehn Geschwistern hat er seine Heimat verlassen und ist nach Italien geflohen, wo er mittlerweile seit fünf Jahren lebt.

Mursal, der zweite Interviewpartner in Italien, ist 37 Jahre alt und in einer großen somalischen Stadt geboren. Er hat in Somalia die Schule besucht und 
eine Frau geheiratet, mit der er drei Kinder hat. Er verließ Somalia aufgrund des Krieges, floh ohne seine Familie über Äthiopien, den Sudan und Libyen und kam als Bootsflüchtling 2008 in Italien an.

\subsection{Mädchenbeschneidung wird als ,normal' beschrieben}

Auf die Frage nach der Rolle der Beschneidung von Mädchen in Somalia sind sich alle männlichen Interviewpartner einig: es ist ,normal'. Jamaal nennt es eine „Routinekultur“, keiner würde über Vor- oder Nachteile der Praktik sprechen, auch er habe lange Zeit nicht darüber nachgedacht. Er glaubt, dass es aus der Angst, Frauen könnten untreu sein, gemacht würde.

I: „So ein bisschen so: dann gehört die Frau nur mir und ich bin mir sicher, dass sie nicht irgendwie mit anderen [Männern...]“"

J: ,Nein, es ist nicht so. Dazu gehört auch, dass sie keine Kinder von einem fremden Mann kriegen bevor sie heiratet und die Männer auch, die denken mit und die denken, wenn die Frau schon mit einem Mann geschlafen hat, dann heiratet die nicht. Das ist eine dämliche und dumme Kultur, natürlich das muss ich sagen." (I 1, 58-63)

Jamaal lehnt Mädchenbeschneidung heute strikt ab, erzählt aber, dass er erst im Laufe seiner Arbeit als Krankenpfleger begriffen habe, wie sehr Frauen unter dem Eingriff leiden würden. Richtig verstanden habe er das Ausmaß der Beschneidung allerdings erst, als er seine Frau heiratete und an ihrem Beispiel gesehen hat, wie sehr sie durch die Beschneidung beeinträchtigt ist. Laut Jamaal würden viele Somalis erst in Deutschland begreifen, dass Beschneidung ihren Mädchen nichts Gutes bringe. In Somalia dagegen würde darüber nicht gesprochen. Daher glaubt Jamaal, dass Mädchenbeschneidung in Somalia niemals abzuschaffen sei. Die Frage, ob gebildete Menschen, sowohl in Somalia als auch immigrierte Somalier_innen, eher auf Mädchenbeschneidung verzichten würden, verneint er.

$\mathrm{J}$ : ,[das liegt nicht an Bildung!] Man ist hierhergekommen, man hört von Familien, von anderen und man kriegt auch das Ganze mit. Sie haben auch diese Erfahrung, diese Schmerzen all das haben die doch auch hinter sich. Wie kann es dann sein, dass eine Mutter, die diese Schmerzen gehabt hat, den eigenen Kindern nochmal diese Schmerzen weitergeben will?" (I 1, 212-115)

Aus seiner Sicht müssten die Menschen, insbesondere die Frauen, von selbst darauf kommen, dass es sich um einen sehr schmerzhaften und folgenreichen Eingriff handelt und ihre Töchter davor bewahren. Dazu bräuchte es Jamaals Meinung nach nicht mehr Bildung, die Erinnerung an den eigenen Schmerz müsste ausreichen, um die Praktik künftig zu unterlassen. Jamaal hat keinerlei Verständnis für Menschen, die in Deutschland leben und ihre 
Töchter beschneiden lassen. Er denkt zwar, dass dies nur noch wenige praktizierten, doch seien das Menschen, mit denen er keinen Kontakt haben wolle.

Auch Abshir berichtet, dass Mädchenbeschneidung in Somalia nicht diskutiert würde. Als Mann wäre man zwar in der Hochzeitsnacht mit den Folgen der Infibulation konfrontiert, aber keiner würde darüber sprechen. Erst in der Schweiz habe er aus Medienberichten von den Konsequenzen der Mädchenbeschneidung erfahren, als die Praktik damals in Europa für großes Aufsehen sorgte. Cigal schildert Mädchenbeschneidung in Somalia als Teil der somalischen ,Kultur', es gehöre dazu, weil unbeschnittene Frauen nicht geheiratet würden. Das läge einfach daran, dass Beschneidung mit Jungfräulichkeit gleichgesetzt würde und Jungfräulichkeit eine große Rolle in Somalia spiele. Insgesamt würde über Mädchenbeschneidung aber nicht diskutiert, es würde schlicht gemacht. Cigal erzählt, dass in Somalia bereits einfache Fragen bezüglich der Beschneidung von Mädchen nicht gestellt würden, da sonst der Eindruck entstehen könne, man stelle die Praktik in Frage. Erst seitdem er in England lebe wisse er, dass Frauenbeschneidung viele Schmerzen mit sich bringe: am Tag der Beschneidung, beim ersten Geschlechtsverkehr und während der Geburt.

\subsection{Aus Sicht der Männer tradieren Frauen die Praktik}

Nahezu alle männlichen Interviewpartner betonen, dass Frauen darüber entscheiden wann und in welcher Form die Töchter beschnitten würden. Feysal berichtet, dass er den Eingriff bei seiner Schwester miterlebt habe und bis heute von der Brutalität, dem vielen Blut und den starken Schmerzen, die seine Schwester hatte, schockiert sei.

F: „Ich habe miterlebt, vor meinen Augen, als die meine älteste Schwester beschnitten haben. Das war fürchterlich. Das war katastrophal. Hab ich, das war zwölf Uhr, da war ich drei oder vier Jahre alt damals und meine Schwester haben vier kräftige Frauen festgehalten: von rechts und links die Schultern und beide Beine und diese Beschneiderin ist gekommen und hat die Klitoris entfernt. Und sie hat VIEL geblutet und sie hat auch mit einem Messer die ähm, ähm [...] einem Messer das nicht scharf ist, ja, geschnitten und meine Schwester hat die ganze Zeit geweint und das habe ich gesehen und habe mitgeweint, ich konnte, bis heute kann ich diese Nacht nicht vergessen." (I 2, 85-94)

Er habe nach diesem Erlebnis seine Eltern mehrfach darum gebeten, die jüngere Schwester vor diesem Eingriff zu bewahren, doch habe niemand auf ihn gehört. Alle seine Schwestern seien infibuliert worden, er habe das nicht verhindern können. Bei seinen eigenen Töchtern habe er sich gemeinsam mit seiner Frau gegen Beschneidung entschieden. Außerdem sei es ihm auch 
gelungen, dass seine Brüder und ein Teil der restlichen Verwandten ihre Töchter unbeschnitten gelassen haben.

Nabiil berichtet ebenfalls, dass es die Frauen seien, die auf die Infibulation beharrten. Er habe dies folgendermaßen erlebt:

$\mathrm{N}$ : „Wenn ich rede, dann rede ich nur von mir, von meinem Clan, von meinen Freunden und Verwandten. Meine älteren Verwandten z.B., sie waren nicht gebildet und sie haben gesagt „Ok, Beschneidung muss stattfinden, keine pharaonische aber sunna." Meine Großmutter, meine Mutter, meine Tante, die haben aber immer gesagt „Nein, man muss die pharaonische durchführen!" Wenn ich von meinem Kreis rede, die waren immer noch dafür. Und meistens in meinem Clan ist es so, wenn der Älteste etwas sagt, dann muss man folgen, dann muss man das machen." (I 3, 91-97)

So hätten sich letztendlich die Frauen durchgesetzt und die Mädchen seien infibuliert worden, da der Rat der Ältesten zu befolgen sei. Bei Nabiils Schwestern sei es ganz ähnlich gewesen, da habe der Vater gesagt, dass sie ,nur' ,sunna' beschnitten werden sollten, doch haben seine Großmutter, Tanten und Mutter sich für die Infibulation entschieden.

Mursal dagegen hat es als Einziger anders erlebt und betont, wie wichtig die Meinung des Mannes sei:

M: „Die Männer, selbst wenn man Ohrlöcher für die Mädchen machen möchte, muss man die Männer um Erlaubnis fragen. Es entscheiden nicht nur die Mütter oder Großmütter, auch die Männer.“ (I 4, 57-59)

Es sei eine Gemeinschaftsentscheidung, die in jedem Fall mit dem Vater abzusprechen sei, so sei es zumindest in seinem Clan gelaufen, berichtet Mursal.

7.3 Wissen um die Konsequenzen des Eingriffs

Die Männer berichteten teilweise sehr detailliert, welche physischen und psychischen Konsequenzen sich aus der Beschneidung ergeben können. Die verheirateten Männer erzählten, die Konsequenzen der Infibulation in der Hochzeitsnacht erlebt zu haben, was keinem der Befragten positiv in Erinnerung geblieben ist. Sie erzählten wie schwierig es war, den vaginalen Geschlechtsverkehr zu vollziehen, und wie schmerzhaft es für ihre Frauen war. Oft habe es mehrere Nächte gedauert bis die Öffnung weit genug war, um vaginalen Geschlechtsverkehr praktizieren zu können.

Mursal berichtet ähnlich von seiner Hochzeitsnacht, beschreibt das Vorgehen als ,normal', da alle Ehepaare in Somalia diese Nacht in dieser Weise erlebten. Manche würden ihre Ehefrau zwar zu einem Arzt bringen, der die Öffnung vor dem ersten Geschlechtsverkehr ein wenig öffnet, er habe das jedoch nicht gemacht, weil er nicht damit gerechnet habe, dass es so schwierig sein würde, eine infibulierte Frauen zu penetrieren. Auch die Komplikationen während Geburt habe er erst nach und nach begriffen. Seine Frau habe die vielen Schmerzen stolz ertragen, erzählt Mursal, weil sie zeigen wollte, dass sie Schmerzen ertragen kann. 
Nabiil erzählt, dass in seinem Clan Mädchen und Jungen gemeinsam beschnitten würden, am selben Tag und oft im selben Raum. Daher habe er mitbekommen, dass Mädchen sich sehr gegen den Eingriff wehren würden, dass sie von mehreren Personen festgehalten werden müssten und laut schreien würden. Er selbst habe auch schon Mädchen festhalten müssen während sie beschnitten worden seien, er wisse aus diesem Grund genau, was bei der Mädchenbeschneidung gemacht würde.

$\mathrm{N}$ : „Ja, klar. Ich war auch schon eingeladen, um die Mädchen zu halten und habe gesehen wie sie das machen. Da habe ich ganz genau geschaut, allerdings war ich danach so durcheinander, weil der hat die Schere gehalten und hat es abgeschnitten wie ein Stück Stoff. Und dann genäht. Und nachher war es so zu, die haben nur so eine kleine Öffnung gelassen. Sie binden danach die Beine zusammen, dann darf sie 15 Tage nicht laufen. Und wenn die Mädchen auf die Toilette gehen zum urinieren, dann schreien sie ganz laut! Ich habe auch mitgekriegt, dass Mädchen viele Schmerzen hatten, wenn sie ihre Tage hatten. Ein Mädchen, das bei uns gewohnt hat, mit dem ich verwandt bin, immer wenn sie ihre Tage hatte, ist sie krank geworden. Und dann mussten wir Medikamente holen und so. Es gibt sooo viele Probleme, wenn man beschnitten ist." (I 3, 61-70)

So hätten die Mädchen am Tag der Beschneidung große Schmerzen, aber auch während ihrer Menstruation und trotzdem würde an der Praktik festgehalten. Aus seinem Leben in Italien berichtet er, dass er Freundinnen habe, die unter starken Menstruationsbeschwerden litten:

M: „Ich habe hier auch ein Mädchen kennen gelernt, dass viel gelitten hat, wenn sie ihre Menstruation hatte. Zwei Tage bevor sie ihre Tage bekommen hat, hat sie höllische Schmerzen bekommen. Dann habe ich sie gefragt „Was hast du sonst immer gemacht, dass das besser wird?“ und sie hat gesagt „Eis auskratzen aus der Gefriertruhe und auf meinen Bauch legen." Als man das Eis aufgelegt hat, ist die Periode geflossen und die Schmerzen wurden besser. Dann hat man ihr gesagt, „Komm wir bringen dich hier zu einem Arzt“ und sie hat gesagt „Nein!“, „Warum?“, weil sie Angst hat, dass man sie aufmacht und sie dann niemandem zum Heiraten findet. Und dann haben wir einen Arzt gefragt, ob es eine Lösung gebe ohne sie aufzuschneiden, dann hat er gesagt: „Heiraten!'““ (I 4, 142-150)

Nabiil erzählt, dass die Angst der Frauen, nicht geheiratet zu werden, groß ist, so groß, dass sie lieber weiterhin die Schmerzen ertragen als sich chirurgisch öffnen zu lassen. Erst nach der Heirat, wenn der Ehemann die Jungfräulichkeit festgestellt und der erste Geschlechtsverkehr stattgefunden hat, ist es möglich, die Frauen ein wenig aufzuschneiden. Nabiil empfindet die Situation in Italien schwierig, da die Frauen insgesamt nicht wüssten wohin mit ihren gesundheitlichen Problemen. Ihm seien keine Anlaufstellen bekannt, die sich um infibulierte Frauen kümmern, auch keine Ärzt_innen. Des Weiteren habe er bei der Begleitung der Frauen oft die Sprachprobleme als größte 
Barriere erlebt. Die Frauen würden zwar ins Krankenhaus gehen, wenn die Schmerzen zu groß würden, doch könne sich dort niemand mit ihnen verständigen, da sie meist kein Italienisch sprechen. Dann würde zwar gesagt, dass sie eine Infektion haben, die medikamentös behandelt werden muss, der Ursprung des Schmerzes sei damit jedoch nicht behoben.

Mursal ist ebenfalls über die Folgen der Infibulation infomiert, betont aber, dass er kein Problem mit der milderen Form, der ,sunna', habe. Mädchen, die nach der milderen Form beschnitten würden, hätten noch Spaß an Sexualität, infibulierte seiner Meinung nach jedoch nicht:

M: „Die beschnittene Frau merkt gar nicht, dass man Sex hat. Sie kaut vielleicht Kaugummi oder sie ist am Telefon und da kannst du machen was $\mathrm{du}$ willst. Ich habe erlebt, dass Frauen Kaugummi kauen und mich fragen, wann ich fertig bin." (I 4, 85-87)

Aus diesem Grund bevorzuge er mittlerweile auch Frauen, die entweder unbeschnitten oder ,sunna' beschnitten seien. Er wolle keine Frauen mehr, die keinen Spaß am Geschlechtsverkehr haben, daher würde er auch schon beim ersten Kontakt danach fragen.

Cigal berichtet, dass Somalis auch in England an der Praktik festhielten. Er schätzt, dass circa 85 Prozent aller in England geborenen Mädchen beschnitten würden. Der Eingriff selbst würde laut Cigal aber in Somalia stattfinden, wo die Mädchen während der Ferien hingebracht würden. Die Familien wüssten, dass der Eingriff in England verboten sei, daher würden sie England lieber verlassen, als auf Beschneidung zu verzichten.

\subsection{Weibliche Genitalbeschneidung als eines von vielen Problemen}

Cigal weiß, dass Frauen teilweise stark unter den Konsequenzen der Infibulation leiden, den vielen Schmerzen, den wiederkehrenden Infektionen und der oft sehr engen Öffnung. Trotzdem spiele Mädchenbeschneidung eine untergeordnete Rolle, da viele Probleme in Somalia existentieller seien. So gäbe es kein Gesundheitssystem, keine funktionierende Regierung, viele gesellschaftlichen Probleme, mangelnde Bildung, kein sauberes Trinkwasser, Krieg und Hungersnöte - über all diese Problemen käme Mädchenbeschneidung und dessen Folgen zu kurz. Ähnlich sieht das auch Nabiil, allerdings aus der Sicht eines Flüchtlings in Italien.

$\mathrm{N}$ : „Ein Flüchtling, der hierher kommt, braucht erst einmal ein Dach über dem Kopf und selbst das haben sie nicht. Medizinische Versorgung und so, das kommt alles an zweiter Stelle. Erst braucht man eine Wohnung. Ich habe so viele Leute gesehen, Mädchen und Jungen, die auf der Straße schlafen, ich habe auch einmal eine Frau mit einem Kind auf der Straße gesehen und es 
war sooo kalt! Dann habe ich die Sanitäter angerufen, die haben aber nicht geholfen, sie haben gesagt, sie können nicht helfen. Dann habe ich ein Hotel gemietet und morgens bin ich sie abholen gegangen und zu den Behörden gebracht und denen erklärt, dass sie keinen Ort zu schlafen hat und ein Kind. Dort hat man ihr einen Zettel gegeben und gesagt „Wir haben gerade keine Wohnung, warten sie bis eine frei wird." Drum gehen sie weg in andere Länder, auch wenn sie den Fingerabdruck hier haben. Weil hier gibt es keine Chance zum Leben, sie können gar nichts erreichen hier. Das ist der Grund warum sie weggehen. Alles andere, gesundheitliche Probleme oder so kommt an zweiter Stelle, erst muss man mal einen Ort zum Schlafen haben." (I 3, 173-184)

Flüchtlinge in Rom würden allein gelassen werden, sie bekämen keinerlei Unterstützung oder wüssten nicht, an welche Anlaufstellen sie sich mit ihren Problemen wenden können. Bevor die Frauen kein Dach über dem Kopf hätten, seien die gesundheitlichen Probleme zweitrangig.

\subsection{Gespräche als Schlüssel im Prozess des Umdenkens}

Auf die Frage, wie man erreichen könne, dass Mädchenbeschneidung unter in England lebenden somalischen Familien abgeschafft werden könne, antwortet Cigal:

C: "TALKING! Educating the people is huge, it's huge you know! And some people they don't realize the problem that the girl will undergo, so they take it easily. They think ( ) so you have to do it to your child, your child will suffer, your child will not forgive you! When she tries to get married, she will not, she will cry so you have to stop it or could you explain to me why it's necessary? Why do you want your child to undergo this process? People will not give you any kind of explanations, good explanation! So they have to think and sit back and say "maybe there is something there!" So talking to the people, facing them in social media, in broadcasting media, in schools, in telling the teachers how to actually (--) training the teachers in how to handle that kind of stuff! It is very important and effective and it could actually stop it and prevent. But without that, it will not!" (I 5, 182-191)

Aus Cigals Sicht ist die Kommunikation mit den Menschen der einzig erfolgsversprechende Weg. Dazu sei es wichtig, in die Community zu gehen, das Gespräch mit den Menschen zu suchen und ihnen zu erklären, warum Mädchenbeschneidung schlecht ist. Dies könnten Somalis tun, doch wären auch Briten wichtig, am besten gemeinsam. Schwierig sei es aber mit den somalischen Männern, da habe er die Erfahrung gemacht, dass vor allem die gebildeten Männer, das heißt diejenigen, die schon andere Kulturen gesehen hätten, in anderen Gesellschaften gelebt hätten, bereit seien, über Mädchen- 
beschneidung zu sprechen. Doch gäbe es von diesen Männern laut Cigal nur wenige, da die somalische Gesellschaft sehr konservativ und unzugänglich sei.

C: "But because we are a very conservative society, Somalian people whether they live in Germany, in Netherlands, in Norway, in England we are socially conservative. If anyone say something other than conservative is wrong. We are a very close society [...].” (I 5, 243-246)

Die Unzugänglichkeit der somalischen Gesellschaft, gepaart mit deren konservativen Einstellung, macht es laut Cigal schwierig, die Menschen, besonders die Männer, zu erreichen und mit ihnen über die Abschaffung der Mädchenbeschneidung zu sprechen. Eines jedoch sei ihm noch wichtig zu sagen: In der somalischen Gesellschaft habe die Frau eine hohe Stellung, sie würde nicht unterdrückt, dürfe selbst über sich und ihr Leben entscheiden. Es seien nicht alleine die Männer, die über die Beschneidung entschieden, vielmehr sei es eine Gemeinschaftsentscheidung. Oft sei es sogar so, dass Frauen stärker an Mädchenbeschneidung festhielten als Männer. Dennoch ist Cigal davon überzeugt, dass mittlerweile eine neue Generation heranwächst, eine, die nicht länger an Mädchenbeschneidung festhalten wird, auch wenn es noch ein langer Prozess sein wird, bis die Praktik unter somalischen Migrant innen in England gänzlich verschwindet.

C: "So it's not actually something which will go easily. It will take some time, it will take a lot of education, it will take a lot of pressure from people like us, you know, the Somalian community, both, and the mainstream society. It is something actually, any human being, like you will actually understand that this is a problem and it's something that should be stopped." (I 5, 322-326)

\subsection{Zusammenfassung der zentralen Ergebnisse}

Die Aussagen der männlichen Interviewpartner zeigen deutlich, dass Mädchenbeschneidung in Somalia als eine stark verwurzelte ,Tradition' präsentiert wird, ein Eingriff, der nicht einfach abzuschaffen ist. Die Männer betonen, dass die Praktik nicht hinterfragt und nicht diskutiert würde. Selbst Fragen zur Mädchenbeschneidung dürften nicht gestellt werden, weil sonst der Eindruck entstehen könne, man sei gegen den Eingriff. In Somalia ist es schlicht ein Tabu, über Mädchenbeschneidung zu sprechen. Im jeweiligen Einwanderungsland verhielte es sich dagegen anders, da wäre es möglich, über Mädchenbeschneidung zu diskutieren, die Praktik in Frage zu stellen und sich dagegen zu entscheiden. In England, der Schweiz und Deutschland habe es sich herumgesprochen, dass es ein verbotener Eingriff sei. Aufklärungsbedarf bestünde dagegen unter somalischen Flüchtlingen, die noch im 
Flüchtlingswohnheim wohnen würden oder erst vor kurzer Zeit angekommen seien. Da sei vermehrt Aufklärung nötig, am besten in der Muttersprache und von einer weiblichen Person.

Dieser Eindruck festigt sich im Hinblick auf Italien. Dort erzählen die beiden Somalis, wie schwer das Überleben als Flüchtlinge in Italien ist. Keiner würde sich um sie kümmern, sie hätten oft keine Unterkunft oder wohnten in baufälligen Häusern ohne Strom und Wasser. Arbeit zu finden sei ebenfalls sehr schwierig. Vor diesem Hintergrund würde Mädchenbeschneidung erst einmal zu einem Randproblem. Dennoch litten Frauen unter den Folgen ihrer Beschneidung, doch wüssten diese Frauen laut meiner Interviewpartner nicht, wohin sie sich mit ihren gesundheitlichen Schwierigkeiten wenden können. Erschwerend käme hinzu, dass es ein sehr intimes und schambesetztes Thema sei, über das die Frauen nicht einfach sprächen. Die Einschätzung der Interviewpartner ist, dass Mädchenbeschneidung zwar nicht an erster Stelle steht, wenn Flüchtlinge immigrieren, es sich jedoch um einen Aspekt handelt, unter dem etliche Frauen leiden, die Unterstützung benötigen.

Aus den Interviews mit den Männern geht auch hervor, dass Mädchenbeschneidung ein gesellschaftliches Problem ist, allerdings hätten laut der Interviewpartner besonders die (älteren) Frauen die Entscheidungsmacht darüber, ob und in welcher Form Mädchen beschnitten würden, weniger die Männer. In Somalia würde aber nicht die Frage diskutiert, ob die Töchter beschnitten werden sollen oder nicht, es wird lediglich über den Typ der Beschneidung gesprochen. Diesbezüglich geben meine Interviewpartner an, dass Frauen stärker zur Infibulation tendierten als Männer. Die Erzählungen der Männer zeigen außerdem, dass auch sie unter der Infibulation ihrer Frauen leiden, was sich besonders in der Hochzeitsnacht niederschlägt. Insgesamt wirke sich, so meine Interviewpartner, die Infibulation auf das Sexualleben aus, da infibulierte Frauen mehr Schmerzen als Spaß beim Geschlechtsverkehr empfinden würden.

Als letzter Punkt ist hervorzuheben, dass alle Interviewpartner die Beschneidung von Mädchen ablehnen, einige von ihnen setzen sich auch dafür ein, die Praktik zu beenden. Im Gegensatz zu den weiblichen Interviewpartnern rechtfertigen die Männer den Eingriff nicht. Sie betrachten die Arbeit gegen Mädchenbeschneidung auch nicht als Angriff auf die somalische ,Kultur', das somalische Volk oder ihre Person. Stattdessen fordern sie noch mehr Aufklärungsarbeit mit dem Ziel, die Menschen schnellstmöglich davon zu überzeugen, ihre Töchter nicht beschneiden zu lassen. Vermutlich ist es den Männern leichter möglich, die Praktik abzulehnen, da sie nur indirekt betroffen sind, sie verkörpern die Beschneidung nicht. Die ,westliche' Darstellung weiblicher Genitalbeschneidung als ,Verstümmelung' bringt Männer nicht unter denselben Rechtfertigungsdruck wie Frauen, da es sich nicht um einen Teil ihrer Identität handelt. Frauen verkörpern die Praktik und so wird jeder Angriff auf Mädchenbeschneidung zu einem Angriff auf sie selbst. 



\section{Zusammenschau der Gesamtergebnisse}

Im nachfolgenden Teil werden die Ergebnisse der quantitativen Untersuchung mit den zentralen Ergebnissen aus den Interviews der somalischen Frauen und Männer zum Thema weibliche Genitalbeschneidung miteinander verbunden und diskutiert.

Im Rahmen der quantitativen Untersuchung stand die Perspektive der Fachkräfte im Vordergrund und folgte der Frage, wie sich der Umgang mit bedrohten und beschnittenen Mädchen und Frauen momentan gestaltet. Die in Kapitel fünf präsentierten Ergebnisse werden nachfolgend nochmals dargelegt und mit den Ergebnissen der qualitativen Interviews verbunden.

Die qualitativen Interviews mit somalischen Frauen basierten auf der Frage, welche Handlungsstrategien beschnittene Frauen entwickelt haben, um mit den Veränderungen umzugehen, die mit dem Verlassen ihres Heimatlandes im Hinblick auf Genitalbeschneidung verbunden sind. Die qualitativen Interviews mit den somalischen Männern zielten darauf ab zu erfahren, welche Sichtweise die männlichen Somalis auf weibliche Genitalbeschneidung haben. Auf Grundlage dieser drei Fragestellungen konnte das Phänomen aus unterschiedlichen Perspektiven betrachtet und die nun folgenden Schlussfolgerungen generiert werden.

\subsection{Genitalbeschneidung als fester Bestandteil bei der Konstruktion von Weiblichkeit}

Aus Sicht der interviewten somalischen Frauen und Männer handelt es sich bei weiblicher Genitalbeschneidung um einen in ihrem Heimatland Somalia als ein ,normal ' betrachteter Eingriff. Begründet wird diese Norm mit der Argumentation, Mädchen würden erst durch diesen Eingriff zu ,reinen“ und ,treuen' Frauen werden. Folgt man der in Kapitel drei aufgezeigten Konstruktion von Weiblichkeit, wird weibliche Genitalbeschneidung in diesem Zusammenhang von der Gesellschaft instrumentalisiert. Ausgehend von der Annahme, das weibliche Genitale sei in seiner natürlichen Anlage fehlerhaft und würde dafür sorgen, dass Frauen ihren sexuellen Trieben ausgesetzt blieben, wird weibliche Genitalbeschneidung zu einem notwendigen Eingriff für heranwachsende Mädchen gesellschaftlich konstruiert. So wird ,normal ${ }^{*}$ mit beschnittenen und vernäht verbunden Genitalien assoziiert und mithilfe machtvoller Diskurse zu einer Schablone (Engel/Schuster 2007, S. 135), die im Gegensatz zu dem Genitale einer unbeschnittenen Frau steht, das mit 
Zuschreibungen wie ,unrein' oder ,sexuell ausschweifend' belegt wird. Den noch unbeschnittenen Mädchen wird der Eingriff als Zeichen der Reinwaschung präsentiert, ohne den sie, schmutzig' oder in anderer Weise fehlerhaft bleiben würden. Die Infibulationsnarbe wird zum Symbol dieser ,Reinheit" und von den Mädchen als erstrebenswerte Norm internalisiert. Normen, die als gesellschaftlich ausgehandelte Regeln $\mathrm{zu}$ verstehen sind, sind wie die Gesellschaft auch von Macht- und Herrschaftsverhältnissen geprägt und gehen mit Normalisierungsprozessen einher (vgl. Engel/Schuster 2007), die unter anderem auch die Vorstellung eines ,normalen' Genitales regulieren. In diesem Diskurs wird das weibliche Genitale zum Ausdruck vermeintlich biologisch determinierter weiblicher Eigenschaften, die es durch den Eingriff zu verhindern oder hervorzurufen gilt - ob Infibulation oder Intimmodifikation, mit dem Eingriff wird Einfluss auf das (sexuelle) Verhalten von Frauen genommen. In diesem Prozess wird der weibliche Körper zu einem ,passi$\mathrm{ve}[\mathrm{n}]$ Medium, dem die kulturellen Bedeutungen eingeschrieben sind" (Butler 2012, S. 26). Das Zurechtschneiden weiblicher Genitalien spiegelt eine gesellschaftlich etablierte Vorstellung von ,normalen' Genitalien wider, die bei den einzelnen Mitgliedern der Gesellschaft zu dem Druck nach Anpassung führt, dem Wunsch aus einem vermeintlich ,abweichenden' Genitale ein ,normales' ${ }^{6}$ zu machen (Vgl. Filter/Reich 2012; Preiß 2010).

Aus den Interviews mit den somalischen Frauen und Männern geht jedoch auch hervor, dass es möglich ist, diese Geschlechtskonstruktionen im Dialog (beispielsweise zwischen Ehepartner_innen) aufzulösen. In Somalia stellt weibliche Genitalbeschneidung eine Norm dar, die momentan nicht verhandelt, sondern unhinterfragt weiter tradiert wird. Im jeweiligen Einwanderungsland aber, so berichten die Interviewpartner_innen, ist es möglich über weibliche Genitalbeschneidung zu sprechen und es wird auch möglich, den Eingriff zu hinterfragen. Im Rahmen dieser Gespräche können häufig genannte Argumente, wie „Männer wollen beschnittene Frauen“ oder „Frauen wären ohne Beschneidung untreu" diskutiert und aufgelöst werden. Die Interviews verdeutlichen, dass diese Gespräche letztlich zu einem Umdenken führen und eine kritische Perspektive im Hinblick auf weibliche Genitalbeschneidung entstehen kann.

Des Weiteren geht aus den Interviews hervor, dass die männlichen Interviewpartner den Eingriff deutlicher ablehnen als die interviewten Frauen, was vermutlich daran liegt, dass Männer den Eingriff nicht mit ihrer Identität verbinden, die Ablehnung somit losgelöst von der eigenen Person erfolgen kann. Die Befragung von Fachkräften der beratenden Einrichtungen und Kliniken zeigt auch, dass die Zielgruppe der Jungen und Männer bislang noch wenig Beachtung finden. Es existieren zwar vereinzelt Angebote für sie, 
doch scheint die Arbeit in Richtung dieser Zielgruppe nur wenig vorangetrieben zu werden.

Neben der Konstruktion weiblicher Genitalbeschneidung als bedeutenden Teil weiblicher Identität wird der Eingriff als Teil der ,Kultur' dargestellt, was sich sowohl in den Äußerungen der Interviewpartner_innen zeigt, wie auch in der Literatur zum Thema weibliche Genitalbeschneidung niederschlägt. Aus postkolonialer Perspektive betrachtet stellt sich die Frage, inwieweit die ,westliche' (feministische) Diskurse dazu beigetragen haben, weibliche Genitalbeschneidung als ,Kultur' oder ,Tradition“ der ,Anderen“ zu konstruieren. Aus, westlicher' Perspektive wird ein Phänomen wie das weiblicher Genitalbeschneidung zu einem ,kulturellen“ Teil der ,Anderen“ konstruiert und als Ausdruck ihrer Rückständigkeit bewertet. Ähnlich wie in Saids (1977) Analyse zur Konstruktion des ,Orients', dessen ,rückständige ‘ Religion aus der Perspektive ,europäischer' Wissenschaftler_innen als Gegenstück zu Europa und dessen ,fortschrittliche' Religion (Castro Varela/Dhawan 2005, S. 30) beschrieben wird, werden Genitalbeschneidung praktizierende Bevölkerungsgruppen zu einer homogenen rückständigen Einheit konstruiert. Eine solche Perspektive bringt mit sich, dass die individuellen Erfahrungen und Umgangsweisen jeder Einzelnen nicht gesehen werden. Stattdessen werden die einzelnen Mitglieder Genitalbeschneidung praktizierender Bevölkerungsgruppen, wie Leiprecht es formuliert, zu „Marionetten, die an den Fäden ihrer Kultur hängen“ (Leiprecht 2004, S. 9) und die angeblich unfähig sind, individuelle Entscheidungen zu treffen. Die Interviews mit den somalischen Frauen und Männern verdeutlichen aber, dass die einzelnen Mitglieder das Phänomen weiblicher Genitalbeschneidung durchaus differenziert betrachten und jede interviewte Person eine eigene Sichtweise und einen eigenen Umgang mit dem Eingriff gefunden hat. Besonders hervorzuheben ist, dass keine der interviewten Personen den Eingriff tatsächlich als Teil somalischer ,Kultur' versteht. Zwar wird weibliche Beschneidung erst einmal ,kulturell' begründet, doch zeigt sich im Verlauf des Gesprächs, dass dies ein vorgeschobenes Argument zu sein scheint, eines, das eventuell genutzt wird, um sich in Abgrenzung zu einer die Praktik ablehnenden Mehrheitsgesellschaft zu positionieren (vgl. Leiprecht 2004, S. 11).

Hinzu kommt, dass das Phänomen in manchen, westlichen' feministischen Diskursen zu dem Zeichen von weiblicher Unterdrückung wird, was sich deutlich in der Benennung des Eingriffs als ,Verstümmelung' ausdrückt. Mit der Bezeichnung, verstümmelt' gehen Zuschreibungen wie ,unvollständig' oder ,krank' einher, die aber im Gegensatz zu dem Bild stehen, das beschnittene Frauen selbst mit ihrer Beschneidung verbinden. Auch wenn selbst betroffene Aktivistinnen dazu aufgerufen haben, den Eingriff als ,Verstüm- 
melung' zu bezeichnen, zeigen die Interviews mit den Frauen deutlich, dass dies nicht für alle beschnittenen Frauen der passende Begriff ist. Schwarze, selbst betroffene Aktivistinnen stehen nicht stellvertretend für alle beschnittenen Frauen. Vielmehr sind sie Zugehörige organisierter, intellektueller, feministischer Gruppen, die sich dem , westlichen' feministischen Diskurs anschließen und gehört werden. Ähnlich wie das bereits beschriebene Beispiel Spivaks zur Witwenverbrennung (Castro Varela/Dhawan 2005, S. 56), wird auch weibliche Genitalbeschneidung von manchen ,westlichen' Feministinnen genutzt, um Frauen als ,Opfer' patriarchaler Strukturen darzustellen. Dies zeigt sich eindrücklich in den Publikationen der Neuen Frauenbewegung seit den 1970er Jahren, (vgl. Braun/Levin/Schwarzbauer 1979; Hulverscheidt 2013, S. 94-108). Diese Argumentation wird von der weißen Mehrheitsgesellschaft dafür genutzt, die vermeintlich rückständigen Bevölkerungsgruppen nach ,westlichem" Vorbild zu ,zivilisieren" (Castro Varela/Dhawan, S. 56; Arndt 2005, S. 26).

\subsection{Konsequenzen einer Migration in ein Genitalbeschneidung ablehnendes Einwanderungsland}

Mit dem Verlassen ihres Heimatlandes verbinden die Interviewpartner_innen sowohl positive wie negative Veränderungen. In erster Linie werden die negativen Erfahrungen benannt, die sie mit Gynäkolog_innen oder Hebammen im jeweiligen Einwanderungsland gemacht haben. Im Zusammentreffen mit genannten Berufsgruppen wird den Frauen bewusst, dass sie als beschnittene Frauen nicht länger der gesellschaftlichen Norm entsprechen, mehr noch: Sie spüren eine ablehnende Haltung gegenüber dem für sie bislang positiv besetzten Eingriff. Die negativen Erfahrungen mit Ärzt innen oder Hebammen, die schockiert auf ihre Genitalien reagieren, schildern die befragten somalischen Frauen als prägende Ereignisse im jeweiligen Einwanderungsland. Diese Schilderungen decken sich mit den Aussagen der von Asefaws interviewten Eritreerinnen (vgl. Asefaw 2008).Der Eingriff hängt eng mit der weiblichen Identität der interviewten Frauen zusammen und wird plötzlich nicht nur infrage gestellt, sondern auch negativ bewertet. Dies kann wie Strenge es beschreibt, zu einem Identitätsverlust führen (Strenge 2013, S. 332). Was im Heimatland noch als Ausdruck von Weiblichkeit positiv internalisiert war, kann nach der Migration als Verlust von Weiblichkeit erlebt werden. In Strenges Untersuchung wird deutlich, dass die Migration in ein Land, das weibliche Genitalbeschneidung ablehnt, zu erneuter Traumatisierung beitragen kann, (Strenge 2013, S. 332 ff.). Strenge verweist daher nicht nur auf die Notwendigkeit, beschnittene Frauen 
psychologisch zu begleiten, sondern auch auf die Erfordernis der „Entwicklung transkultureller Konzepte für die kultursensible medizinische, psychiatrische und psychotherapeutische Versorgung“" (zit. n. Feldmann 2012, S. 273 in: Strenge 2013, S. 335) betroffener Frauen zur Verhinderung weiterer Traumatisierung oder Retraumatisierung. Neben den spezifischen Schwierigkeiten, die sich für beschnittene Frauen in einem Genitalbeschneidung ablehnenden Land ergeben können, ist im Allgemeinen darauf hinzuweisen, dass Migration ,als ein psychisch belastender und über mehrere Generationen reichender Prozess" (Reinprecht/Weiss 2012, S. 16) angesehen werden muss, da sich Migrant_innen einerseits von ihrer Herkunftsgesellschaft entfremden, gleichzeitig auf Widerstände in der Aufnahmegesellschaft stoßen (ebd.). Der psychisch belastende Prozess der Migration wird demnach für beschnittene Frauen durch die ablehnende Haltung gegenüber weiblicher Genitalbeschneidung zusätzlich erschwert. Trotz der Ergebnisse dieser Untersuchungen bleiben beschnittene Frauen im Umgang mit den psychischen Konsequenzen noch weitestgehend alleine, da, wie die quantitative Untersuchung im Rahmen dieser Forschungsarbeit gezeigt hat, noch zu wenig Begleitung von beschnittenen Frauen durch qualifiziertes psychologisches Fachpersonal möglich ist. Darüber hinaus wird aus der quantitativen Erhebung und den Interviews mit den Frauen deutlich, dass noch zu wenige Gynäkolog_innen existieren, die über die Konsequenzen weiblicher Genitalbeschneidung informiert sind, ein Ergebnis, das sich mit den Ergebnissen der Dissertation Grafs (2012) deckt. Ihre Ergebnisse haben ebenfalls gezeigt, dass die befragten Gynäkolog_innen in Berlin über unzureichendes Wissen bezüglich des Umgangs mit beschnittenen Frauen verfügen und ebenso wenig über die Hintergründe genitaler Beschneidung informiert sind (Graf 2012, S. 147 ff.). Auch die Ergebnisse Asefaws (2008) zeigen, dass ihre Interviewpartnerinnen bemängelten, noch häufig auf uniformiertes medizinisches Fachpersonal zu treffen (Asefaw 2008, S. 74). Um eben genannte (Re-)traumatisierung in Zukunft zu verhindern, ist die Aufklärung von Ärzt_innen und Hebammen zum Umgang mit beschnittenen Frauen von Bedeutung. Dieser zeichnet sich nicht nur durch die Kenntnis bestimmter physischer oder psychischer Konsequenzen aus, sondern auch durch die den Frauen entgegengebrachte Haltung. In Anlehnung an die Erkenntnisse postkolonialer Studien und der Kritischen Weißseinsforschung ist besonders darauf zu achten, den Eingriff nicht als Zeichen ihrer Rückständigkeit zu werten und betroffenen Frauen nicht herabzustufend und mit einer belehrenden Haltung zu begegnen, sondern dafür zu sorgen, dass Gespräche frei von Wertung und auf gleichberechtigter Ebene geführt werden.

Die Migration bringt neben der genannten negativen Erfahrung jedoch auch mit sich, dass ein Hinterfragen weiblicher Genitalbeschneidung möglich wird. Sowohl die interviewten Männer als auch die Frauen erzählen, dass im Rahmen der gesellschaftlichen Strukturen in Somalia Gespräche über weibli- 
che Genitalbeschneidung nicht möglich gewesen seien. Erst im Einwanderungsland hätten sie über den Eingriff und dessen Folgen diskutieren und damit einen Prozess des Umdenkens einleiten können. Die mit diesen Gesprächen verbundenen Informationen zu den Konsequenzen des Eingriffs werden als zentrales Moment im Prozess des Umdenkens benannt. Auch die Angaben der Beratungsstellen und Kliniken zeigen, dass Informationsveranstaltungen und Gesprächsrunden in der Arbeit gegen weibliche Genitalbeschneidung von großer Bedeutung sind. Ebenso wird von Seiten der Einrichtungen bestätigt, dass es sinnvoll ist, diese Veranstaltungen innerhalb der Communities zu organisieren und dafür zu sorgen, dass sie in der jeweiligen Muttersprache stattfinden können. Die Migration in ein Genitalbeschneidung ablehnendes Land birgt somit die Chance, die Beendung von Mädchenbeschneidung zu forcieren. Die im Rahmen dieser Untersuchung interviewten Frauen und Männer haben sich gegen die Beschneidung ihrer Töchter entschieden, eine Entscheidung, die im Heimatland vermutlich gegenteilig ausgefallen wäre. Dies zeigen auch die Ergebnisse der Studie in Hamburg, in der die interviewten Familien sich deutlich gegen die Genitalbeschneidung aussprachen und ebenfalls angaben, ihre Töchter nicht beschneiden zu wollen (Behrendt 2010, S. 97 ff.).

Ein weiterer Aspekt, der im Rahmen dieser Forschungsarbeit deutlich wurde, ist, dass die interviewten Personen über das gesetzliche Verbot von weiblicher Genitalbeschneidung im jeweiligen Einwanderungsland informiert waren. Auch die befragten Beratungsstellen benannten das Gesetz in der Arbeit gegen Genitalverstümmelung als hilfreich, ein Ergebnis, das sich mit vorangegangenen Studien deckt (vgl. Asefaw 2008; Behrendt 2010) und zeigt, dass die gesetzliche Situation ebenfalls zum Schutz bedrohter Mädchen beitragen kann. Gleichzeitig verdeutlicht die Untersuchung Behrendts (2010) aber, dass die Familien, die auf die Notwendigkeit der Beschneidung ihrer Töchter bestehen, den Eingriff im jeweiligen Heimatland durchführen. Dies geben auch die somalischen Interviewpartner_innen an. Die gesetzliche Regelung scheint somit für im Ausland begangene Beschneidungen nicht $\mathrm{zu}$ greifen. Diejenigen, die ihre Töchter beschneiden lassen wollen, umgehen die gesetzliche Regelung, indem sie den Eingriff im Heimatland durchführen lassen, was im Einwanderungsland weder kontrolliert noch geahndet wird. 


\section{$6 \quad$ Konsequenzen für Politik und Beratung}

Die Ergebnisse der im Rahmen dieser Forschungsarbeit durchgeführten Untersuchungen lassen Schlussfolgerungen zu, die sowohl für die Politik als auch die pädagogische, psychologische und medizinische Beratung und Begleitung bedrohter und beschnittener Mädchen und Frauen von Bedeutung sind. Nachfolgend werden die sich aus den Ergebnissen ableitenden Konsequenzen dargestellt und begründet.

\subsection{Finanzielle Förderung von Migrant_innenvereinen}

Die Ergebnisse der quantitativen Erhebung zeigen deutlich, dass Migrant innenvereine weniger Schwierigkeiten haben, Kontakt zu bedrohten und betroffenen Mädchen und Frauen und deren Familien herzustellen. Den Mitarbeiter_innen der Migrant_innenvereine ist in diesen Gesprächen möglich, die Beratung und Aufklärung in der jeweiligen Muttersprache durchzuführen, was auch die interviewten Frauen als wichtigen Gesichtspunkt in der Aufklärungsarbeit gegen weibliche Genitalbeschneidung benennen. Die Befragung der Einrichtungen verdeutlicht jedoch auch, dass Migrant innenvereine nur über wenig finanzielle Mittel verfügen. Dies bringt mit sich, dass die meiste Arbeit ehrenamtlich oder auf Honorarbasis durchgeführt wird und am Ende nicht zur Sicherung des Lebensunterhaltes ausreicht. Im Hinblick darauf, dass diese Einrichtungen den besten Kontakt zu bedrohten und betroffenen Mädchen und Frauen herstellen können und somit dafür sorgen, dass eine umfassende Beratung und Begleitung der Zielgruppe möglich wird, ergibt sich die politische Forderung, diese Einrichtungen in Zukunft finanziell zu unterstützen.

\subsection{Aufnahme der Thematik in die medizinische, psychologische und pädagogische Ausbildung}

Die steigende Anzahl von genital beschnittenen Frauen nach Europa bringt mit sich, dass mit dem Phänomen weiblicher Genitalbeschneidung umgegangen werden muss. Eine bedeutende Rolle spielen dabei Berufsgruppen, die im Laufe ihrer Tätigkeit möglicherweise in Kontakt mit beschnittenen Frauen oder von Beschneidung bedrohten Mädchen kommen werden. Dies betrifft 
vor allem Ärzt_innen, Hebammen und Krankenschwestern/-pfleger. Laut Aussagen der interviewten Frauen wird beschnittenen im Kontakt mit Gynäkolog_innen oder Hebammen erstmals bewusst, dass weibliche Genitalbeschneidung, im Besonderen die Infibulation, medizinische Schwierigkeiten mit sich bringen kann. Diese Untersuchungs- und/oder Behandlungssituation eröffnet genannten Berufsgruppen die Möglichkeit, betroffene Frauen zu den Konsequenzen des Eingriffs und den medizinischen Umgang damit aufzuklären und sie während des Prozesses zu begleiten. Da dies nur gelingen kann, wenn medizinische Fachkräfte im Umgang mit der Thematik geschult sind, ist die Aufnahme derselben in die Curricula von Medizinstudent innen und in die Ausbildung von Hebammen unbedingt erforderlich. Die Aufnahme des Themas und des Umgangs mit beschnittenen Frauen, ist nicht allein wichtig, um ihnen eine adäquate medizinische Behandlung zu ermöglichen. Sie sorgt auch dafür, in Zukunft emotionale Reaktionen von Gynäkolog_innen und/oder Hebammen zu vermeiden und Betroffene dadurch nicht bereits beim ersten Aufeinandertreffen zu verunsichern. Ein weiterer Vorteil ist, dass auf diese Weise auch zum Schutz bedrohter Mädchen beigetragen werden kann. Gynäkolog_innen, Hebammen oder Pädiater_innen stehen während er Schwangerschaft und in der Zeit danach in besonders engem Kontakt mit den Frauen. Hat eine beschnittene Frau ein Mädchen entbunden, kann dieser Kontakt auch zu Gesprächen genutzt werden, um die Beschneidung ihrer Töchter zu verhindern. Pädiater_innen müssen des Weiteren ihrer Pflicht nachkommen, im Rahmen der verpflichtenden Kindervorsorgeuntersuchungen die Genitalien der Kinder zu untersuchen, eine Aufgabe, die sie bei jedem Kind haben. Kindern aus Genitalbeschneidung praktizierenden Bevölkerungsgruppen kommt deshalb keine diskriminierende Sonderstellung zu, da die Untersuchung der Genitalien nicht nur die Beschneidung weiblicher Genitalien verhindern oder aufdecken kann, sondern andere Krankheitsbilder oder auch sexuellen Missbrauch. Ferner ist die Aufnahme der Thematik in die Curricula von Pädagogikstudierenden und Erzieher_innen wichtig, da diese, je nach Arbeitsfeld, ebenfalls im Kontakt mit Kindern und Jugendlichen und deren Familien stehen. Das Wissen um weibliche Genitalbeschneidung und das Vorgehen im Verdachtsfall kann zum Schutz bedrohter Mädchen beitragen. Den Ergebnissen der Interviews ist weiter zu entnehmen, dass die Aufnahme der Thematik in das Curriculum des Faches Psychologie wichtig wäre, um betroffenen Frauen in Zukunft die Möglichkeit zu geben, psychologische Begleitung im Umgang mit den psychischen Folgen des Eingriffs nutzen zu können. Die Entscheidung einer solchen psychologischen Begleitung obliegt natürlich den Frauen, doch sollte im Kontakt mit Betroffenen bedacht werden, dass die Konfrontation mit den negativen Konsequenzen, die Frauen psychisch belasten kann. 


\subsection{Schulungen für Mediziner_innen, Psycholog_innen und Pädagog_innen im Umgang mit beschnittenen Frauen}

Für dieselben Berufsgruppen gilt es, Schulungsangebote zu schaffen, um sie mit der Thematik vertraut zu machen. Im Rahmen dieser Schulungen ist ebenso wichtig, die Teilnehmer_innen auch im Umgang mit betroffenen und deren Familien auszubilden Die unterschiedlichen Umgangsweisen der interviewten Frauen zeigen, dass ganz individuell entschieden werden muss, welche Art von Unterstützung Betroffene benötigen. Manche benötigen erst einmal eine umfassende, bewertungsfreie Aufklärung zu möglichen Konsequenzen, andere konkrete medizinische und/oder psychologische Unterstützung. Neben der Vermittlung medizinischer und psychologischer Konsequenzen des Eingriffs, sollte im Rahmen der Schulungen, aber auch im Rahmen der Ausbildungsgänge, besonderes Augenmerk auf der den Frauen und Männern entgegengebrachte Haltung gelegt werden. Teilnehmer_innen sollte vermittelt werden, dass Gespräche zu dieser Thematik frei von Wertung sein sollten und den Gesprächspartner_innen auf Augenhöhe zu begegnen ist. Die Gespräche sollten in erster Linie dazu da sein, die Position Betroffener zu verstehen, nicht sie von Anfang an davon zu überzeugen, dass ihre Position schlecht ist. Schließlich sollte vermieden werden, Betroffene und ihr Umfeld einem Unterlegenheitsgefühl auszusetzen oder sie in Bedrängnis zu bringen, sich für ihre Norm rechtfertigen zu müssen. Bilder, wie ,bemitleidenswerte Opfer' oder eine problematisierende Haltung der Praktik gegenüber, lösen bei Betroffenen eventuell Rechtfertigungsdruck oder Ablehnung aus. Möchte man erreichen, dass beschnittene Frauen die Informationen annehmen und die Notwendigkeit des Eingriffs hinterfragen, ist ein offener und wertschätzender Kontakt wichtig. Drohungen und Verbote sind kontraproduktiv, stattdessen muss den Frauen erklärt werden, warum Genitalbeschneidung negativ für sie und ihre Töchter ist. Des Weiteren ist zu berücksichtigen, dass die Veränderung sozialer Normen Zeit benötigt. Dies bedeutet für die Arbeit gegen weibliche Genitalbeschneidung, dass es sich um ein Phänomen handelt, das sich nicht in kurzer Zeit beenden lassen wird. Druckausübung oder Verbote alleine werden nicht dazu führen, einen reflexiven Prozess in der Bevölkerung anzuregen. Vielmehr zeigen die Interviews mit den somalischen Frauen, aber auch die Untersuchungen Asefaws (2008) oder Behrendts (2004), dass die Menschen aus weibliche Genitalbeschneidung praktizierenden Ländern häufig nicht wissen, dass der Eingriff negative Folgen mit sich bringen kann (vgl. Asefaw 2008, S. 41 ff.; Behrendt 2010, S. 97 ff.). Aufklärungsarbeit muss also in erster Linie bedeuten, ihnen die Konsequenzen weiblicher Genitalbeschneidung aufzuzeigen. Diesbezüglich sind offene und wiederholte Gespräche nötig, um mögliche negative Konsequenzen des Eingriffs zu verdeutlichen. Des Weiteren ist das gesamte soziale Umfeld be- 
schnittener Frauen in die Aufklärungsarbeit zu den Konsequenzen des Eingriffs einzubeziehen - inklusive der männlichen Mitglieder. Wiederkehrende Gespräche (gegebenenfalls in geschlechtshomogenen Gruppen) über die negativen Folgen des Eingriffs, vor allem aber die Betonung, dass es keine religiöse Pflicht darstellt, Mädchen beschneiden zu lassen, sind zur Abschaffung der Praktik nötig.

\subsection{Offenheit gegenüber neuen Wegen in der Beratung}

Ein wichtiger Aspekt, der besonders die bereits bestehenden Beratungsstellen und deren Angebote betrifft, ist die von den Interviewpartner_innen wiederholt genannte Information, dass es wichtig ist, die Arbeit gegen weibliche Genitalbeschneidung in einen anderen Kontext einzubetten. Betroffene und deren Familien ließen sich eher auf eine Informationsveranstaltung ein, die zu Themen wie reproduktiver Gesundheit, Erziehung, oder sonstigen Themen stattfinden würde. Die Thematik zentral zu platzieren, sei kontraproduktiv, das würde die Menschen abschrecken.

Die Arbeit der Migrant_innenvereine verdeutlicht weiter, dass es sinnvoll ist, mehr in die Familien und die Communitys zu gehen, um Aufklärungsarbeit zu leisten. Eine Beratungsstelle aufzusuchen und sich dort Hilfe zu holen, ist nicht für alle Betroffene ein gangbarer Weg, vielleicht aus Angst nicht verstanden zu werden oder aus Scham oder aus Unwissenheit über die Existenz solcher Einrichtungen. Ein weiterer Vorteil der Arbeit in den Familien oder Communitys ist, dass auf diese Weise auch die männlichen (Familien)Mitglieder erreicht werden können. Im Hinblick darauf, dass die somalischen Interviewpartner_innen angeben, im Einwanderungsland über weibliche Genitalbeschneidung diskutieren zu können, wäre es wichtig, die männlichen Mitglieder gegebenenfalls mit mehr Informationen zu den Konsequenzen des Eingriffs zu versorgen, um sie in ihrer Ablehnung zu stärken, damit sie sich in Gesprächen mit Befürworter_innen der Praktik besser durchsetzen können. So könnten sie nicht nur im Hinblick auf ihre eigenen Töchter, sondern auch als Vorbild oder als Vermittler zwischen einzelnen männlichen Mitgliedern der Communities oder einzelnen Familien genutzt werden. Vor dem Hintergrund, dass die Befragung der Einrichtungen ergeben hat, dass bedrohte Mädchen mit Angeboten nur schwer erreicht werden, spielen beide Elternteile beim Schutz bedrohter Mädchen eine wichtige Rolle. Insgesamt sollte vermehrt auf die Einbindung männlicher Gegner der Praktik in die Arbeit geachtet werden, da es ihnen, so wie es die männlichen Interviewpartner auch erzählen, in vielen Fällen leichter fällt, die Männer zu erreichen. Auch die Einbindung von Imamen kann in der Arbeit gegen Genitalbeschneidung vorteilhaft sein, um zu verdeutlichen, dass weibliche Genitalbe- 
schneidung keine religiöse Pflicht darstellt, so wie dies eine Einrichtung in Italien beschreibt.

Um den Kontakt zu Betroffenen und deren sozialem Umfeld herzustellen, ist der Einsatz so genannter interkultureller Vermittler_innen von Nutzen. In einigen Fällen, so hat die Untersuchung gezeigt, wird darauf auch zurückgegriffen, doch scheint dies ein Potential zu sein, das noch zu wenig genutzt wird. Der Einsatz dieser interkulturellen Vermittler innen sollte nicht an bestimmte Ausbildungs- oder Studienabschlüsse gekoppelt sein. Vielmehr sollte die Kompetenz im Vordergrund stehen, mit Menschen in einen offenen Dialog treten und die Gespräche in der jeweiligen Muttersprache abhalten zu können. Das sollten künftig jedoch nicht ausschließlich eine ehrenamtliche Tätigkeiten sein: Interkulturelle Vermittler_innen müssen für ihre Arbeit finanziell entlohnt werden.

\subsection{Beratung in Flüchtlingswohnheimen}

Bei der Aufklärungsarbeit gegen weibliche Genitalbeschneidung ist die besondere Lage von Flüchtlingen und asylsuchenden Personen zu beachten. Die Untersuchungen haben gezeigt, dass keine Einrichtung angegeben hat, in die Flüchtlingswohnheime zu gehen, um Mädchen und Frauen bezüglich geschlechtsspezifischer Fluchtgründe aufzuklären. Die Interviews mit somalischen Flüchtlingen in Italien haben ergeben, dass es sich um eine besonders bedürftige Zielgruppe handelt, da sie teilweise um ihr Überleben kämpfen müssen. Die Situation der Flüchtlinge ist sicher nicht repräsentativ für alle Flüchtlinge der untersuchten Einwanderungsländer. Dennoch benennen die Interviewten Schwierigkeiten, die für alle Flüchtlinge gelten, wie zum Beispiel wenig Chancen auf Arbeit zu haben oder eine Wohnung zu bekommen. Auf diese Weise wäre darüber hinaus sichergestellt, dass Familien aus Genitalbeschneidung praktizierenden Bevölkerungsgruppen darüber informiert sind, dass der Eingriff im Einwanderungsland verboten ist, weil er schwere Konsequenzen für Mädchen und Frauen nach sich ziehen kann.

In Bezug auf bedrohte und beschnittene Mädchen und Frauen ist jedoch besonders bedeutsam, dass drohende Genitalbeschneidung $\mathrm{zu}$ einem anerkannten Asylgrund werden muss. Die vorliegende Forschungsarbeit hat gezeigt, dass es sich bei dem Eingriff um eine gesellschaftlich etablierte Norm handelt, der sich kaum entzogen werden kann. Entscheiden sich Frauen oder Eltern dafür, ihre Töchter vor diesem Eingriff zu schützen, ist dies im jeweiligen Heimatland - trotz gesetzlicher Verbote - kaum möglich, was bereits etliche andere Studien und Veröffentlichungen gezeigt haben. 


\section{Fazit und Ausblick}

Der zentrale Ausgangspunkt dieser Forschungsarbeit, verschiedene Perspektiven auf weibliche Genitalbescheidung aufzuzeigen, konnte mithilfe der jeweiligen Untersuchungen realisiert werden und liefert ein facettenreiches Bild. Die quantitative Befragung der Beratungsstellen zur zentralen Frage, ob beziehungsweise wie bedrohte und betroffene Mädchen und Frauen bestehende Angebote nutzen, hat ergeben, dass Migrant innenvereine und Einrichtungen, die von (selbst betroffenen) Migrant_innen aus Genitalbeschneidung praktizierenden Bevölkerungsgruppen geleitet werden, die genannte Zielgruppe am besten erreichen. In diesem Zusammenhang ist die Möglichkeit der Durchführung von Aufklärungs- und Beratungsgesprächen in der jeweiligen Muttersprache besonders vorteilhaft. Des Weiteren hat die Untersuchung gezeigt, dass die Zielgruppe der Jungen und Männer weiterhin vernachlässigt wird, obwohl alle Einrichtungen, Aufklärungsarbeit für Jungen und Männer als wichtigen Ansatzpunkt in der Arbeit gegen weibliche Genitalbeschneidung empfinden. Zuletzt wurde deutlich, dass bislang noch $\mathrm{zu}$ wenige Gynäkolog_innen und Psycholog_innen existieren, die im Umgang mit den physischen wie psychischen Konsequenzen beschnittener Frauen geschult sind. Besonders der Mangel an Psycholog_innen hat zur Folge, dass Beschnittene kaum Möglichkeiten haben, sich psychologisch begleiten zu lassen. Die überwiegende Mehrheit der Beratungsstellen wünscht sich diesbezüglich mehr Schulungen für genannte Berufsgruppen sowie eine engere Vernetzung zwischen Einrichtung und niedergelassenen Gynäkolog_innen und Psycholog_innen.

Die qualitativen Interviews zur Beleuchtung der Perspektive beschnittener Frauen haben deutlich gemacht, dass es sich bei weiblicher Genitalbeschneidung in Somalia um eine gesellschaftlich etablierte Norm handelt, die eng an die weibliche Identität gekoppelt ist. Die Migration in ein Einwanderungsland hat zur Folge, dass beschnittene Frauen erstmals die Möglichkeit haben, diese Norm zu hinterfragen. Dieses erstmalige Hinterfragen dieser Norm, kann Vor- und Nachteile für beschnittene Frauen mit sich bringen. Mit dem Verlassen des gesellschaftlichen Kontexts verlieren die im Zusammenhang mit der Konstruktion von weiblicher Identität geltenden Begründungen für den Eingriff ihre Bedeutung. Es ist möglich, dass der Eingriff ohne den gesellschaftlichen Kontext als Verlust der weiblichen Identität empfunden wird, ein Verlust, der sich in psychischen Leiden manifestieren kann. Der Vorteil, den die Migration in ein Genitalbeschneidung ablehnendes Einwanderungsland mit sich bringt, ist, dass die Praktik erstmals diskutiert werden kann. Die Interviews mit den somalischen Frauen und Männern haben verdeutlicht, dass diese Gespräche, ob mit dem/der Ehepartner_in oder unter Freunden, ein wichtiges Element im Umdenkprozess darstellt. Wo es in Somalia noch ein 
Tabu war, über weibliche Genitalbeschneidung und deren Konsequenzen zu sprechen, ist dies im Einwanderungsland möglich und kann, wie bei den im Rahmen dieser Arbeit interviewten Personen, dazu führen, dass die im Einwanderungsland aufwachsenden Töchter unbeschnitten bleiben. Des Weiteren haben die Interviews gezeigt, dass die den Frauen und Männern entgegengebrachte Haltung von großer Bedeutung ist, soll Aufklärungs- und Beratungsarbeit die Zielgruppe erreichen. Im Kontakt mit Betroffenen und deren Familien ist darauf zu achten, diesen mit Wertschätzung, Offenheit und auf gleichberechtigter Ebene zu begegnen.

Abschließend kann festgehalten werden, dass die Untersuchungen im Rahmen dieser Forschungsarbeit Antworten auf die formulierten Forschungsfragen liefern konnten. Gleichzeitig ist in der Reflexion des gesamten Forschungsprozesses aber auch darauf hinzuweisen, dass von Vorteil gewesen wäre, die Zahl der untersuchten Einwanderungsländer geringer zu halten. In einer Untersuchung, die sich neben Deutschland noch auf maximal ein weiteres Einwanderungsland beschränkt hätte, wäre ein detaillierter Einblick in die Strukturen des Einwanderungslandes und die Perspektive der interviewten Personen möglich gewesen und trotzdem wäre eine vergleichende Perspektive auf unterschiedlich strukturierte Einwanderungsländer und deren Möglichkeiten und Begrenzungen für Migrant_innen beziehungsweise Flüchtlinge aus Somalia möglich gewesen.

Aus der vorliegenden Forschungsarbeit geht außerdem hervor, dass die Erforschung der psychischen Auswirkungen weiblicher Genitalbeschneidung, insbesondere im Kontext von Migration, weiter vorangetrieben werden muss. Diesbezüglich wären breit angelegte Studien mit einem gemischten Sample nötig, um ein möglichst differenziertes Bild der psychischen Situation beschnittener Frauen zeichnen zu können. Dies erscheint im Hinblick auf die Ergebnisse der wenigen Studien, die zu den psychischen Auswirkungen existieren, wichtig, da diese gezeigt haben, dass ein Teil beschnittener Frauen unter psychischen Konsequenzen (wie der Traumatisierung, die der Eingriff mit sich bringt und/oder der Traumatisierung, die sich aus der Migration in ein weibliche Genitalbeschneidung ablehnendes Einwanderungsland ergeben kann) leidet, jedoch bislang nicht mit entsprechender Begleitung versorgt wird. Des Weiteren wäre es wichtig, bestehende Beratungsangebote der existierenden Einrichtungen - national wie international - regelmäßig zu evaluieren, um diese an die Bedürfnislagen bedrohter und beschnittener Mädchen und Frauen anzupassen. Auf Basis dieser Evaluationen könnten die einzelnen Einrichtungen voneinander profitieren und gemeinsam nachhaltige Ansätze entwickeln, die neben den betroffenen und bedrohten Mädchen und Frauen auch die männlichen Familienmitglieder erreichen und dafür sorgen, bedrohte Mädchen künftig vor dem Eingriff zu schützen. 



\section{Transkriptionssystem}

Die Regeln wurden in Anlehnung an das GAT (Gesprächsanalytisches Transkriptionssystem) erstellt. Das Transkriptionssystem wurde vereinfacht, da manche Interviews von einer Dolmetscherin ins Deutsche übersetzt und transkribiert wurde, weshalb das System nicht zu viele Zeichen enthalten sollte, um die Transkription nicht zusätzlich zu erschweren.

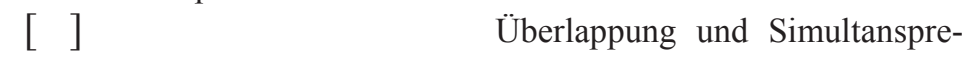

chen

( - ), ( -- ), ( --- ) Kurze, mittlere, längere Pause

äh, öh, etc.

sog. Gefüllte Pausen

unverständliche Passage je nach

Länge

\{hustend\}

sprachbegleitende Handlungen

oder Ereignisse

$\{\{$ erstaunt $\}\} \quad$ interpretierende Kommentare

So (h) o

Lachen während des

Redens

(lacht)

Lautes, spontanes Lachen

(lächelt)

leises Lächeln während

des Erzählens 
GROßBUCHSTABEN Betonung bestimmter Wörter oder Silben, lauter sprechen an bestimmten Stellen

Transkriptionsregeln verfügbar unter:

https://www.ph-freiburg.de/quasus/einstiegstexte/datenaufbereitung /transkriptionsregeln.html

(Stand: 09. Juli 2014) 


\section{Literaturverzeichnis}

Abdeleli, Abdelhafidh (2013): Somalier auf der Suche nach Integration. Verfügbar unter:

http://www.swissinfo.ch/ger/politik_schweiz/Somalier_auf_der_Suc he_nach_Integration.html?cid=37118000 (Stand: 06. Februar 2014).

Arndt, Susann (2004): Kolonialismus, Rassismus und Sprache.

Verfügbar unter:

http://www.bpb.de/gesellschaft/migration/afrikanischediaspora/59407/afrikaterminologie (Stand: 05. Mai 2014).

Arndt, Susan (2005): Weißsein. Die verkannte Strukturkategorie Europas und Deutschlands. In: Arndt, Susan/Eggers, Maureen Maischa/Kilomba, Grada/Piesche, Peggy (Hg.): Kritische Weißseinsforschung. Mythen, Masken und Subjekte. Münster Unrast Verlag, S. 24-30.

Arndt, Susan (2006): „The Racial Turn“: Kolonialismus, weiße Mythen und Critical Whiteness Studies. In: Bechhaus-Gerst, Marianne/Gieseke, Sunna (Hg.): Afrika und Europa. Koloniale und Postkoloniale Begegnungen. Frankfurt/Main: Peter Lang Verlag, S.11-27.

Assefaw, Fana (2007): Female Genital Cutting (FGC) - Eine Feldstudie unter besonderer Berücksichtigung der Hintergründe sowie der gesundheitlichen und psychosexuellen Folgen für Betroffene und Partner in Eritrea und Deutschland. Universität Witten/Herdecke.

Assefaw, Fana (2008): Weibliche Genitalbeschneidung. Hintergründe, gesundheitliche Folgen und nachhaltige Prävention. Königstein/Taunus: Ulrike Helmer Verlag.

Bauer, Christina/Hulverscheidt, Marione (2003): Gesundheitliche Folgen der weiblichen Genitalverstümmelung. In: Terre des Femmes (Hg.): Schnitt in die Seele. Frankfurt/Main: Mabuse Verlag S.65-81.

Baumann, Mechthild (2014): Frontex - Fragen und Antworten. Verfügbar unter:

http://www.bpb.de/gesellschaft/migration/kurzdossiers/179679/front ex-fragen-und-antworten (Stand: 30. Juni 2014).

Bäßler, Annegret/Wachall, Bernhard/Ender, Jens (2006): Eine Frage der Herrschaft. Somalia zwischen Krieg und Frieden in der zweiten Hälfte des 20. Jahrhunderts. Norderstedt: GRIN Verlag.

Behrendt, Alice (2004): Das Vorkommen Posttraumatischer Belastungsstörung nach weiblicher Genitalbeschneidung. Unveröffentlichte Diplomarbeit. Universität Hamburg.

Behrendt, Alice (2010): Listen to Africain Voices. Female Genital Mutilation/Cutting among Immigrants in Hamburg: Knowledge, Attitudes and Practice. Plan International (Hg.) Hamburg. 
Berger, Peter/Luckmann, Thomas (1969): Die gesellschaftliche Konstruktion der Wirklichkeit. Eine Theorie der Wissenssoziologie. Frankfurt/Main: S. Fischer Verlag.

Birnbaum, Michael (2002): Krisenherd Somalia. Das Land des Terrors und der Anarchie. München: Wilhelm Heyne Verlag.

Bielefeld, Heiner (2007): Menschenrechte in der Einwanderungsgesellschaft. Plädoyer für einen aufgeklärten Multikulturalismus. Bielefeld: Transcript Verlag.

Borkenhagen, Ada (2010): Designervaginas oder das geschönte Geschlecht. In: Borkenhagen, Ada, Brähler, Elmar (Hg.): Intimmodifikationen. Spielarten und ihre psychosozialen Bedeutungen. Band 95. Gießen: Psychosozial - Verlag, S. 97-113.

Böhm, Andreas: Theoretisches Codieren. In: Flick, Uwe/von Kardorff, Ernst/Steinke, Ines (Hg.): Qualitative Forschung. Ein Handbuch. Reinbeck bei Hamburg: Rowohlt Taschenbuch Verlag, S. 475-485. Braun, Ingried/Levin, Tobe/Schwarzbauer, Angelika (Hg.) (1979): Materialien zur Unterstützung von Aktionsgruppen gegen Klitorisbeschneidung. München: Verlag Frauenoffensive.

Buddeberg, Claus (2003): Psychosoziale Medizin. Berlin: Springer Verlag.

Büchner, Anna Christin (2004): Weibliche Genitalverstümmelung. Betrachtungen eines traditionellen Brauchs aus Menschenrechtsperspektive. Schlussfolgerungen für die Soziale Arbeit in Deutschland. Oldenburg: Paulo Freire Verlag.

Bundesamt für Migration und Flüchtlinge (2013): Glossar. Verfügbar unter: http://www.bamf.de/DE/Service/Left/Glossary/_function/glossar.ht ml?lv2=1364186\&lv3=3198544 (Stand: 19. Oktober 2013).

Bundesamt für Statistik (2013): Bevölkerung und Erwerbstätigkeit. Verfügbar unter:

https://www.destatis.de/DE/Publikationen/Thematisch/Bevoelkerun g/MigrationIntegration/AuslaendBevoelkerung2010200137004.pdf? blob= publicationFile (Stand: 08. Mai 2014).

Bundesärztekammer (2013): Empfehlungen zum Umgang mit Patientinnen nach weiblicher Genitalverstümmelung. Verfügbar unter: http://www.bundesaerztekammer.de/downloads/Empfehlungen_Gen italverstuemmelungen_20134.pdf (Stand: 10. April 2014).

Bundesministerium für Justiz und für Verbraucherschutz (1992): Asylverfahrensgesetz (AsylVFG). Verfügbar unter:

http://www.gesetze-iminternet.de/asylvfg_1992/BJNR111260992.html (Stand 09. September 2014)

Bundeszentrale für politische Aufklärung (2014): Frontex. Verfügbar unter: http://www.bpb.de/nachschlagen/lexika/176988/frontex (Stand: 03. Mai 2014) 
Bundeszentrale für politische Aufklärung (2014): Somalia. Verfügbar unter: http://www.bpb.de/nachschlagen/lexika/fischerweltalmanach $/ 65795 /$ somalia? $\mathrm{p}=7$ (Stand 04. Mai 2014).

Butler, Judith (2012): Die Macht der Geschlechternormen und die Grenzen des Menschlichen. Frankfurt/Main: Suhrkamp.

Butterwegge, Carolin (2005): Migration in Ost- und Westdeutschland von 1955 bis 2004. Verfügbar unter:

http://www.bpb.de/gesellschaft/migration/dossier-migration/56367 /migration-1955-2004 (Stand: 05. Mai 2014).

Carell, Noemi (2013): Die liberale Migrationspolitik des jungen Bundesstaates. Verfügbar unter:

http://www.bpb.de/gesellschaft/migration/laenderprofile/139688/migrationsp olitik (Stand: 05. Mai 2014).

Carell, Noemi (2012): Flucht und Asyl. Verfügbar unter:

http://www.bpb.de/gesellschaft/migration/laenderprofile/139690/flucht-undasyl (Stand: 05. Mai 2014).

Castro Varela, Maria do Mar/Nikita Dhawan (2005): Postkoloniale Theorie. Eine kritische Einführung. Bielefeld Transcript Verlag.

Castro Varela, Maria do Mar/Nikita Dhawan (2010): Mission Impossible: Postkoloniale Theorie im deutschsprachigen Raum? In: Reuter, Julia/Villa, Paula-Irene (Hg.): Postkoloniale Studien und Soziologie. Empirische Befunde, theoretische Anschlüsse, politische Intervention. Bielefeld: Transcript Verlag, S. 303-330.

Crenshaw, Kimberle (1998): Demarginalizing the Intersection of Race and Sex: A Black Feminist Critique of Antidiscrimination Doctrine, feminist Theory and Antiracist. In: Feminism in the Law: Theory, Practice and Criticism. The University of Chicago Legal Forum 1989, S.139-167.

Denzin, Norman K. (2004): Symbolischer Interaktionismus. In: Flick, Uwe/von Kardorff, Ernst/Steinke, Ines (Hg.): Qualitative Forschung. Ein Handbuch. Reinbek bei Hamburg: Rowohlt Taschenbuch Verlag, S. 136-150.

Deutsches Institut für Menschenrechte (2013): „Racial Profiling“ - menschenrechtswidrige Personenkontrollen nach §22 Abs.1a Bundespolizeigesetz 2013.Verfügbar unter:

http://www.institut-fuer-menschenrechte.de/uploads/tx_commerce/ Stu-

die_Racial_Profiling_Menschenrechtswidrige_Personenkontrollen_ nach_Bundespolizeigesetz.pdf (Stand: 25. Mai 2014).

Dini, Shukria (2008): Geschlechterbeziehungen, Gesellschaft und Politik in Somalia. In: Heinrich Böll Stiftung (Hg.): Somalia. Alte Konflikte und neue Chancen zur Staatsbildung. Berlin: Heinrich Böll Stiftung, Schriften zur Demokratie, Band 6, S.99-122. 
Di Muzio, Giorgia (2012): Migrationspolitik. Verfügbar unter: http:/www.bpb.de/gesellschaft/migration/laenderprofile/145671/mi grationspolitik (Stand: 07. Mai 2014).

Dirie, Asili (2003): Betroffene Frauen verdienen unseren Respekt und unsere Unterstützung. In: Terre des Femmes (Hg.): Schnitt in die Seele. Frankfurt/Main: Mabuse Verlag, S.101-109.

Dorndales de Andrade, Daniela/Jirovsky, Elena/Paloni, Sara (2010): Kosmetische Eingriffe und weibliche Genitalverstümmelung. In: Borkenhagen, Ada/Brähler, Elmar (Hg.): Intimmodifikationen. Spielarten und ihre psychosozialen Bedeutungen. Band 95. Gießen: Psychosozial - Verlag, S. 167-182.

Edwards, Viv (2007): Somali today. Verfügbar unter: http://www.bbc.co.uk/voices/multilingual/somali.shtml (Stand: 06. Februar 2013)

Engel, Antke/Schuster, Nina (2007): Die Denaturalisiserung von Geschlecht und Sexualität. Queer/feministische Auseinandersetzung mit Foucault. In: Anhorn, Rohland/Bettinger, Frank/Stehr, Johannes (Hg.): Foucaults Machtanalytik und Soziale Arbeit. Eine kritische Einführung und Bestandsaufnahme. Wiesbaden: Verlag für Sozialwissenschaften, S.135-155.

Eggers, Maureen M.: Rassifizierte Machtdifferenz als Deutungsperspektive in der Kritischen Weißseinsforschung in Deutschland.In: Arndt, Susan/Eggers, Maureen Maischa/Kilomba, Grada/Piesche, Peggy (Hg.) (2005): Kritische Weißseinsforschung. Mythen, Masken und Subjekte. Münster: Unrast Verlag, S.56-73.

Esser, Hartmut (2001): Soziologie. Spezielle Grundlagen. Band 6: Sinn und Kultur. Frankfurt/Main: Campus Verlag.

Flick, Uwe/von Kardorff, Ernst/Steinke, Ines: Was ist qualitative Forschung? Einleitung und Überblick. In: dies. (Hg.) (2004): Qualitative Forschung. Ein Handbuch. Reinbek bei Hamburg: Rowohlt Taschenbuch Verlag, S. 13-29.

Frankfurter Allgemeine Zeitung (2012): Hassan Sheikh Mohamud ist neuer Präsident. Verfügbar unter: http://www.faz.net/aktuell/politik/ausland/somalia-hassan-sheikhmohamud-ist-neuer-praesident-11885842.html (Stand: 27. März 2013).

Foucault, Michel (1991): Der Wille zum Wissen. Sexualität und Wahrheit 1. Frankfurt/Main: Suhrkamp Verlag.

Glaser, Barney G./Strauss, Anselm L. (2008): Grounded Theory. Strategien qualitativer Forschung. Bern: Verlag Hans Huber.

Graf, Jana: Weibliche Genitalverstümmelung und die Praxis in Deutschland.Hintergründe - Positionierung zur Ethik - ärztliche Erfahrungen. Friedrich-Alexander-Universität Erlangen-Nürnberg. 
Guesnet, Maria (2010): Arbeitspapier Postkoloniale Theorie. Afrikabilder Vorstellungen von Afrika im europäischen (insbesondere deutschen) Kontext. Begriffe Stereotypisierungen und Akteure. Verfügbar unter:

http://afrikabilder.blogsport.de/images/AP_Postkoloniale_Theorie.p df (Stand: 16. September 2013).

Hall, Stuart (1994): The West and the Rest. Discourse and Power. Verfügbar unter:

http://analepsis.files.wordpress.com/2013/08/hall-west-the-rest.pdf

Hansen, Randall (2007a): Hintergrundinformationen. Verfügbar unter: http://www.bpb.de/gesellschaft/migration/laenderprofile/57954/hinte rgrund (Stand: 05. Juli 2014).

Hansen, Randall (2007b): Die Entwicklung der Zuwanderungspolitik. Verfügbar unter:

http://www.bpb.de/gesellschaft/migration/laenderprofile/57958/zuw anderungspolitik (Stand: 30. Juni 2014).

Hansen, Randall (2007c): Multikulturalismus. Verfügbar unter: http://www.bpb.de/gesellschaft/migration/laenderprofile/57966/mult ikulturalismus (Stand 08. Juli 2014).

Hermann, Conny (Hg.) 2000: Das Recht auf Weiblichkeit. Hoffnung im Kampf gegen Genitalverstümmelung. Bonn: Dietz.

Hulverscheidt, Marione (2002): Weibliche Genitalverstümmelung. Diskussion und Praxis in der Medizin während des 19. Jahrhunderts im deutschsprachigen Raum. Frankfurt/Main: Mabuse Verlag.

Hulverscheidt, Marione (2013): Health right or human right? Changing tides in the international discussion of Female Genital Mutilation, 19702010. In: Mold, Alex/David Reubi (Hg.): Health Rights in Global Context: Contemporary and Historical Perspectives. London und New York: Routledge 2013, S. 94-108.

Hulverscheidt, Marione/Ahlers, Christoph J./Ihring, Isabelle (2009): Weibliche Genitalverstümmelung - soziokulturelle Hintergründe, rechtliche Rahmenbedingungen, gesundheitliche Folgen, Möglichkeiten der Intervention. Ein Übersichtsbeitrag für die sexualmedizinische und ärztliche Praxis. In: Zeitschrift Sexuologie Heft1/2, S. 17-32.

Ihring, Isabelle (2006): Menschenrechtspädagogik als Bildungs- und Aufklärungsarbeit in Beratungsstellen. Zum Umgang mit weiblicher Genitalverstümmelung in Deutschland und Frankreich. Unveröffentlichte Diplomarbeit, Pädagogische Hochschule Freiburg.

Jablonski, Nina (2014): Rasse war schon immer ein vages und schlüpfriges Konzept. Verfügbar unter: http://de.richarddawkins.net/foundation_articles/2014/2/3/ninajablonski-rasse-war-schon-immer-ein-vages-und-schl-pfrigeskonzept (Stand. 16. August 2014) 
Kalpaka, Annita (2005) : Pädagogische Professionalität in der Kulturalisierungsfalle Über den Umgang mit ,Kultur' in Verhältnissen von Differenz und Macht. In: Leiprecht, Rudolf/Kerber, Anne (Hg.): Schule in der Einwanderungsgesellschaft. Ein Handbuch. Schwalbach am Taunus: Wochenschauverlag, S. 387-406.

Kalthegener, Regina (2003): Strafrechtliche Regelungen in europäischen Staaten. In: Terre des Femmes (Hg.): Schnitt in die Seele. Frankfurt/Main: Mabuse Verlag, S.187-194.

Kelle, Udo/Erzberger, Christian (2004): Qualitative und quantitative Methoden: kein Gegensatz. In: Flick, Uwe/von Kardorff, Ernst/Steinke, Ines (Hg.): Qualitative Forschung. Ein Handbuch. Reinbek bei Hamburg: Rowohlt Taschenbuch Verlag, S.299-309.

Kilomba, Grada (2009): Das N-Wort. Verfügbar unter: http://www.bpb.de/gesellschaft/migration/afrikanischediaspora/59448/ das-n-wort (Stand: 05. Mai 2014).

Lewis, Ioan (2008): Understanding Somalia and Somaliland. Culture, History, Society. London: HURST Publisher Ltd.

Lightfoot-Klein, Hanny (1992): Das grausame Ritual. Frankfurt/Main: Fischer Taschenbuch Verlag.

Lightfoot-Klein, Hanny (2003): Der Beschneidungsskandal. Berlin: Orlanda Frauenverlag.

Leiprecht, Rudolph (2004): Kultur - Was ist das eigentlich? Verfügbar unter: http://www.uni-oldenburg.de/fileadmin/user_upload /paedagogik/personen/rudolf.leiprecht/Kulturtextveroeffentl..pdf (Stand: 08. August 2014)

Lenz, Ilse (2008): Frauenbewegungen: Zu den Anliegen und Verlaufsformen von Frauenbewegungen als sozialen Bewegungen. In: Becker, Ruth/Kortendiek, Beate (Hg.) (2008): Handbuch Frauen- und Geschlechterforschung. Theorie, Methoden, Empirie. Wiesbaden: Verlag für Sozialwissenschaften, S.665-675.

Leye, Els (2008): Female Genital Mutilation. A study of health services and legislation in some countries of the European Union. Dissertation. Universität Gent.

Lorey, Isabell (2008): Kritik und Kategorie. Zur Begrenzung politischer Praxis durch neuere Theoreme der Intersektionalität, Interdependenz und Kritischen Weißseinsforschung. Verfügbar unter: http://eipcp.net/transversal/0806/lorey/de (Stand: 06. Mai 2014).

Mayring, Philipp (1999): Einführung in die qualitative Sozialforschung. 2. Auflage. Weinheim und Basel: Beltz Verlag.

Mecheril, Paul (2004): Einführung in die Migrationspädagogik. Weinheim und Basel: Beltz Verlag.

Meier-Braun, Karl-Heinz (2013): Deutschland Einwanderungsland. In: Meier-Braun, Karl-Heinz/Weber, Reinhold (Hg.): Deutschland Einwan- 
derungsland. Begriffe Fakten Kontroversen. Stuttgart: Verlag W. Kohlhammer, S. 15-27.

Mende, Janne (2011): Begründungsmuster weiblicher Genitalverstümmelung. Zur Vermittlung von Kulturrelativismus und Universalismus. Bielefeld: Transkript Verlag.

Meßmer, Anna-Katharina (2012): Der Kampf um die Vulva hat begonnen. In: Filter, Dagmar/Reich, Jana: „Bei mir bist du schön...“Feministisches Forum. Hamburger Texte zur Frauenforschung. Band 4., Verlagsort: Verlag, S. 119-135.

Muckel, Petra (1998): Selbstreflexivität und Subjektivität im Forschungsprozess. S.61-79. Verfügbar unter:

http://www.qualitativeforschung.de/information/publikation/modelle/breuer/breuer.pdf (Stand: 20. September 2014)

Mutschler, Alexander (2001): Eine Frage der Herrschaft. Betrachtungen zum Problem des Staatszerfalls in Afrika am Beispiel Äthiopiens und Somalias. Wien: LIT Verlag.

Netzwerk Flüchtlingsforschung (2013): Migrationsregime. Verfügbar unter: http://fluechtlingsforschung.net/veranstaltung/migrationsregime-vor-ortlokale-migrationsregime/ (Stand: 28. Juli 2014).

Oltmer, Jochen (2013a): Migration. In: Meier-Braun, Karl-Heinz/Weber, Reinhold (Hg.) (2013): Deutschland Einwanderungsland. Begriffe Fakten Kontroversen. Stuttgart: Verlag W. Kohlhammer, S. 31-34.

Oltmer, Jochen (2013b): Die politische Ordnung der globalen Migrationsverhältnisse in Gegenwart und Zukunft. Verfügbar unter:

http://www.bpb.de/gesellschaft/migration/kurzdossiers/168597/migrationspol itik (Stand: 10. Juli 2014).

Oltmer, Jochen (2005): Deutsche Migrationsgeschichte seit 1871. Verfügbar unter: http://www.bpb.de/gesellschaft/migration/dossiermigration/56355/migration-1871-1950 (Stand: 30. Juni 14).

Preiß, Simone (2010): Plastische Korrekturen im weiblichen Genitalbereich. In: Borkenhagen, Ada/Brähler, Elmar (Hg.): Intimmodifikationen. Spielarten und ihre psychosozialen Bedeutungen. Band 95. Gießen: Psychosozial Verlag, S.81-96.

Preuß, Roland (2014): So sieht der Asylkompromiss aus. Verfügbar unter: http:/www.sueddeutsche.de/politik/herkunftsstaaten-und-residenzpflicht-sosieht-der-asylkompromiss-aus-1.2137533 (Stand: 19.September 2014)

Pro Asyl (2014): Titel. Verfügbar unter:

http://www.proasyl.de/de/themen/basics/basiswissen/rechte-der-

fluechtlinge/ (Stand: 05. Mai 2014).

Prolongeau, Hubert (2006): Victoire sur l'excision. Paris : Albin Michel. 
Rebeggiani, Fatma (2013): Migrationspolitische Ergebnisse des Koalitionsvertrags. Verfügbar unter:

http://www.bpb.de/gesellschaft/migration/newsletter/175159/migrati onspolitik-im-koalitionsvertrag (Stand: 30. Juni 2014).

Rebeggiani, Fatma (2014): Neue Regeln für Frontex. Verfügbar unter:

http://www.bpb.de/gesellschaft/migration/newsletter/184551/europaeischeunion-neue-regeln-fuer-frontex (Stand: 30. Juni 2014).

Reed-Andersson, Paulette (2004): Ein Platz an der afrikanischen Sonne. Verfügbar unter:

http://www.bpb.de/gesellschaft/migration/afrikanische-diaspora/ 59372/kolonialgeschichte (Stand: 05. Mai 2014).

Reinprecht, Chistoph/Weiss, Hilde (2011): Migration und Integration: Soziologische Perspektive und Erklärungsansätze. In: Fassmann,

Heinz/Dahlvik, Julia (Hg.): Migrations- und Integrationsforschung multidisziplinäre Perspektiven. Göttingen: Vandenhoeck \& Ruprecht, S. S.13-34.

Reuter, Julia/Villa, Paula-Irene (2010): Provincializing Soziologie. Postkoloniale Theorie als Herausforderung. In: Reuter, Julia/Villa, PaulaIrene (Hg.): Postkoloniale Studien und Soziologie. Empirische Befunde, theoretische Anschlüsse, politische Intervention. Bielefeld: Transcript Verlag, S. 11-46.

Richter, Gritt/Schnüll, Petra (2003): Einleitung. In: Terre des Femmes (Hg.) (2003): Schnitt in die Seele. Frankfurt/Main: Mabuse Verlag, S. 1520.

Riegel, Christine (2012): Intersektionalität und Jugendforschung. Verfügbar unter: www.portal-intersektionalität.de (Stand: 07. März 2014)

Runkel, Gunter (2005): Allgemeine Soziologie. München und Wien: Oldenbourg Verlag.

Sanyal, Mithu M. (2009): Vulva. Die Enthüllung des unsichtbaren Geschlechts. Berlin: Klaus Wagenbach Wissenschaftsverlag.

Sauer, Birgit (2006): Gender und Sex. In: Scherr, Albert (Hg.): Soziologische Basics Eine Einführung für Pädagogen und Pädagoginnen. Wiesbaden: VS Verlag für Sozialwissenschaften, S. 50-56.

Schicho, Walter (2004): Handbuch Afrika. Nord- und Ostafrika. Frankfurt/Main Brandes \& Apsel.

Schmidt-Grunert, Marianne (1999): Sozialarbeitsforschung konkret. Problemzentrierte Interviews als qualitative Erhebungsmethode. Freiburg/Breisgau: Lambertus Verlag.

Schneider, Jan (2007a): Rückblick: Zuwanderungsgesetz 2005. Verfügbar unter: http://www.bpb.de/gesellschaft/migration/dossiermigration/56351/zuwanderungsgesetz-2005 (Stand: 05. Mai 2014). 
Schneider, Jan (2007b): Die Novellierung des Zuwanderungsgesetzes 2007. Verfügbar unter:

http://www.bpb.de/gesellschaft/migration/dossier-migration/ 56350/zuwanderungsgesetz-2007 (Stand: 05. Mai 2014).

Schnüll, Petra (2003): Weibliche Genitalverstümmelung in Afrika. In: Terre des Femmes (Hg.): Schnitt in die Seele. Frankfurt/Main: Mabuse Verlag, S.23-64.

Schütze, Fritz (1983): Biographieforschung und narratives Interview. In. Neue Praxis 13 (1983), S. 283-293. Verfügbar unter: http://www.ssoar.info/ssoar/bitstream/handle/document/5314/ssoarnp-1983-3-schutze-

biographieforschung_und_narratives_interview.pdf (Stand: 26. August 2014)

Schwarz, Heide (2013): Der tägliche Kampf ums Überleben. Zur Situation der Flüchtlinge in Italien. Verfügbar unter: http://www.amnesty-hamburg.de/index.php/fluechjtlinge-undasyl/244-der-taegliche-kampf-ums-ueberleben (Stand: 14. Juni 2014).

Statistic Netherlands. Verfügbar unter: http://statline.cbs.nl/StatWeb/publication/?DM=SLEN\&PA=37325e $\mathrm{ng} \& \mathrm{D} 1=\mathrm{a} \& \mathrm{D} 2=\mathrm{a} \& \mathrm{D} 3=0 \& \mathrm{D} 4=0 \& \mathrm{D} 5=192 \& \mathrm{D} 6=11-$ $17 \& L A=E N \& V W=T$ (Stand: 06. Februar 2013).

Strenge, Katharina Doris (2013): Traumatisierung durch weibliche Genitalverstümmelung. Ein Prozessmodell unter besonderer Berücksichtigung der Migration. In: Trauma \& Gewalt 7, Heft 4/2013, S. 324337.

Strübin, Jörg (2004): Grounded Theory. Zur sozialtheoretischen und epistemologischen Fundierung des Verfahrens der empirisch begründeten Theoriebildung. Wiesbaden: Verlag für Sozialwissenschaften.

Süddeutsche Zeitung (2009): Verfolgt von einem Verdacht. Verfügbar unter: http://jetzt.sueddeutsche.de/texte/anzeigen/463107 (Stand: 14. Juni 2009).

Schweizer, Katinka/Richter-Appelt, Hertha (Hg.) (2012): Intersexualität kontrovers. Grundlagen, Erfahrungen, Positionen. Gießen: Psychosozial Verlag.

Terre des Femmes (Hg.) (2005): Studie zur weibliche Genitalverstümmelung (FGM). Verfügbar unter: http://frauenrechte.de/online/images/downloads/fgm/EU-StudieFGM.pdf (Stand: 18. Dezember 2012).

Terre des Femmes (Hg.) (2013): Dunkelziffer zur weiblichen Genitalverstümmelung in Deutschland. Verfügbar unter: http://www.frauenrechte.de/online/images/downloads/fgm/StatistikFGM.pdf (Stand: 10. Januar 2014) 
Toubia, Nahid (1994): Female Circumcision as a public Health Issue. In New England Journal for Medicine 331, S. 712-716.

Tsianos, Vassilis (2010): Zur Geneaologie und Praxis des Migrationsregimes. In: Bildpunkt. Zeitschrift der IG Bildende Kunst. Wien, Frühling 2010 „Regimestörungen“. Verfügbar unter: http://www.igbildendekunst.at/bildpunkt/2010/regimestoerungen/tsi anos.htm (Stand: 20. Mai 2014).

UN Committee on the Elimination of Discrimination Against Women (CEDAW), CEDAW General Recommendation No. 14: Female Circumcision, 1990, A/45/38 and Corrigendum. Verfügbar unter: http://www.refworld.org/docid/453882a30.html (Stand:02. August 2014).

UNHCR (2012): UNHCR Global Trends 2012: Displacement. The new $21^{\text {st }}$ Century Challenge. Verfügbar unter: http://www.uno-fluechtlingshilfe.de/fileadmin/redaktion/ PDF/UNHCR/GlobalTrends2012.PDF (Stand 17. März 2014).

UNHCR (Jahr): Fragen und Antworten: Flüchtling. Verfügbar unter: http://www.unhcr.de/questions-und-answers/fluechtling.html (Stand: 05. Mai 2014).

UNIFEM (Jahr): Verfügbar unter : https://www.unwomen.de/themen/beendigung-der-gewalt-gegenfrauen/ null-toleranz-gegenueber-weiblichergenitalverstuemmelung.html (Stand: 06.Oktober 2013).

UNO-Flüchtlingshilfe (2013): Zahlen und Fakten 2013. Verfügbar unter: http://www.uno-fluechtlingshilfe.de/fluechtlinge/zahlen-fakten.html (Stand: 17. März 2014).

Villa, Paula-Irene (2011): Sexy Bodies. Wiesbaden: VS Verlag für Sozialwissenschaften.

Villa, Paula-Irene (2008): (De)Konstruktion und Diskurs-Genealogie: Zur Position und Rezeption von Judith Butler. In: Becker, Ruth/Kortendiek, Beate (Hg.): Handbuch Frauen- und Geschlechterforschung. Theorie, Methoden, Empirie. Wiesbaden: Verlag für Sozialwissenschaften, S.141-152.

Walgenbach, Katharina (2012): Intersektionalität - eine Einführung. Verfügbar unter: www.portal-intersektionalität.de (Stand: 06. März 2014).

Winker, Gabriele/Degele, Nina (2010): Intersektionalität. Zur Analyse sozialer Ungleichheiten. Bielefeld: Transcript Verlag.

Witzel, Andreas (2000): Das problemzentrierte Interview [25 Absätze]. Forum Qualitative Sozialforschung / Forum: Qualitative Social Research, 1(1), Art. 22. Verfügbar unter: http://nbn-resolving.de/urn:nbn:de:0114-fqs0001228 (Stand: 26. August 2013). 
World Health Organisation (2014): Female Genital Mutilation. Verfügbar unter:

http://www.who.int/mediacentre/factsheets/fs241/en/index.html (Stand: 06.04.2014)

Zeit Online (2014): Frontex wird zur Rettung von Flüchtlingen verpflichtet. Verfügbar unter: http://www.zeit.de/politik/ausland/2014-

02/frontex-eu-grenze-fluechtlinge-hilfe (Stand: 07. Mai 2014)

Zinsou Agbota, Sénouvo (2005): Ein Fremder, wer’s glaubt! Klischées da, wo man sie am wenigsten erwarten würde. In: Arndt, Susan/Eggers, Maureen Maischa/Kilomba, Grada/Piesche, Peggy (Hg.): Kritische Weißseinsforschung. Mythen, Masken und Subjekte. Münster: Unrast Verlag, S.221-237. 


\section{Gender - theoretisch}

Katharina Walgenbach

Gabriele Dietze

Lann Hornscheidt

Kerstin Palm

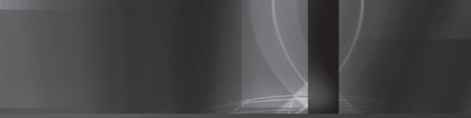

Gender als

interdependente Kategorie

Neue Perspektiven auf Intersektionalität,

Diversität und Heterogenität

2. uuchiggeshene Auflage
Katharina Walgenbach

Gabriele Dietze

Lann Hornscheidt

Kerstin Palm

Gender als interdependente

Kategorie

2., durchgesehene Aufl. 2012. 192 Seiten. Kart. $19,90 €(D), 20,50 €(A)$ ISBN 978-3-86649-496-1

Aus der Perspektive unterschiedlicher Fachbereiche präsentiert dieser Band eine theoretische Diskussion der Zusammenhänge zwischen Geschlecht, Sexualität, Ethnizität, Schicht etc. Ein Buch für alle, die sich mit Konzepten von Diversity sowie mit Gender beschäftigen.

Jetzt in Ihrer Buchhandlung bestellen oder direkt bei:

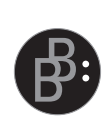

Verlag Barbara Budrich Barbara Budrich Publishers Stauffenbergstr. 7 51379 Leverkusen-Opladen

Tel +49 (0)2171.344.594

Fax +49 (0)2171.344.693 info@budrich.de

www.budrich-verlag.de 


\section{Soziale Arbeit als}

wissenschaftliche Disziplin

\section{Bi utb.}

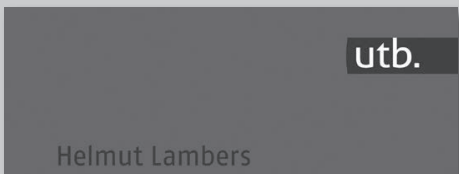

Theorien der Sozialen Arbeit

\section{Ein Kompendium}

und Vergleich

2. Auflage
Helmut Lambers

Theorien der

Sozialen Arbeit

Ein Kompendium und Vergleich

utb $\mathrm{M}$

2., überarb. Auflage 2015.

365 Seiten. Kart.

$24,99 €(D), 25,70 €(A)$

ISBN 978-3-8252-4322-7
Soziale Arbeit hat sich als wissenschaftliche Disziplin etabliert. Sie verfügt indessen nicht über eine verbindliche Zentraltheorie. Studierende Sozialer Arbeit müssen sich mit verschiedenen Konzeptionen disziplin- und professionstheoretischer Reflexion auseinandersetzen. Theoriebildung Sozialer Arbeit muss als Theoriediskurs verstanden werden.
Helmut Lambers führt in die komplexe Theorielandschaft ein und sorgt für die nötige Orientierung. Neben der Einführung in die verschiedenen Theorien nimmt er einen Theorienvergleich vor.

Jetzt in Ihrer Buchhandlung bestellen oder direkt bei: www.utb-shop.de utb GmbH I Industriestr. 2 I 70565 Stuttgart 


\section{Schreiben mit Plan}

\section{B? utb.}

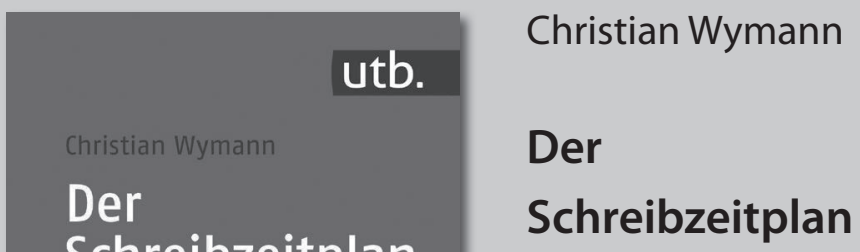

Schreibzeitplan

Zeitmanagement

für Schreibende

utb $S$

2015. 120 Seiten. Kart. $9,99 €(D), 10,30 €(A)$

ISBN 978-3-8252-4308-1

Studierende und Wissenschaftler/ innen sind vor allem eins: Schreibende. Wer aber mit den eigenen Schreibgewohnheiten unzufrieden ist, dem kann ein Schreibzeitplan helfen, eigene Projekte leichter und schneller umzusetzen.
Zehn Schritte genügen, einen eigenen Schreibzeitplan aufzubauen und durchzuhalten. Schreibgewohnheiten werden identifiziert und hilfreiche Schreibroutinen entwickelt. Die Kontrolle über die eigene Schreibsituation kehrt zurück.

Jetzt in Ihrer Buchhandlung bestellen oder direkt bei: www.utb-shop.de utb GmbH I Industriestr. 2 I 70565 Stuttgart 